\title{
TRANSPORT PARALLÈLE ET CORRESPONDANCE DE SIMPSON $p$-ADIQUE
}

\author{
DAXIN XU \\ Laboratoire Alexander Grothendieck, ERL 9216 du CNRS, \\ Institut des Hautes Études Scientifiques, 35 route de Chartres, \\ 91440 Bures-sur-Yvette, France; \\ email: daxinxu@ihes.fr
}

Received 21 juin 2016; accepted 19 mars 2017

\begin{abstract}
Résumé
Deninger et Werner ont développé un analogue pour les courbes $p$-adiques de la correspondance classique de Narasimhan et Seshadri entre les fibrés vectoriels stables de degré 0 et les représentations unitaires du groupe fondamental topologique pour une courbe complexe propre et lisse. Par transport parallèle, ils ont associé fonctoriellement à chaque fibré vectoriel sur une courbe $p$-adique, dont la réduction est fortement semi-stable de degré 0 , une représentation $p$-adique du groupe fondamental de la courbe. Ils se sont posé quelques questions : leur foncteur est-il pleinement fidèle? La cohomologie des systèmes locaux fournis par celui-ci admet-elle une filtration de Hodge-Tate? Leur construction est-elle compatible avec la correspondance de Simpson $p$-adique développée par Faltings? Nous répondons à ces questions dans cet article.
\end{abstract}

\begin{abstract}
Deninger and Werner developed an analogue for $p$-adic curves of the classical correspondence of Narasimhan and Seshadri between stable bundles of degree 0 and unitary representations of the topological fundamental group for a complex smooth proper curve. Using parallel transport, they associated functorially to every vector bundle on a $p$-adic curve whose reduction is strongly semi-stable of degree 0 a $p$-adic representation of the fundamental group of the curve. They asked several questions: whether their functor is fully faithful; whether the cohomology of the local systems produced by this functor admits a Hodge-Tate filtration; and whether their construction is compatible with the $p$-adic Simpson correspondence developed by Faltings. We answer these questions in this article.
\end{abstract}

2010 Mathematics Subject Classification: 14H60 (primary); 11G20, 14G20 (secondary)

(c) The Author 2017. This is an Open Access article, distributed under the terms of the Creative Commons Attribution licence (http://creativecommons.org/licenses/by/3.0/), which permits unrestricted re-use, distribution, and reproduction in any medium, provided the original work is properly cited. 


\section{Introduction}

1.1. Narasimhan et Seshadri [36] ont établi en 1965 une correspondance bijective entre l'ensemble des classes d'équivalence de représentations unitaires irréductibles du groupe fondamental topologique d'une surface de Riemann compacte $X$ de genre $\geqslant 2$ et l'ensemble des classes d'isomorphisme de fibrés vectoriels stables de degré 0 sur $X$. Deninger et Werner ont développé récemment un analogue partiel pour les courbes $p$-adiques [13]. Leur construction est basée sur la notion de fibré vectoriel fortement semi-stable sur une courbe en caractéristique $p>0$. Rappelons qu'un fibré vectoriel sur une courbe $C$ propre et lisse sur un corps algébriquement clos de caractéristique $p$ est dit fortement semi-stable si ses images inverses par toutes les puissances entières du Frobenius absolu de $C$ sont semi-stables.

1.2. Soit $K$ un corps de valuation discrète complet de caractéristique 0 de corps résiduel une clôture algébrique d'un corps fini $\mathbb{F}_{p}, \bar{K}$ une clôture algébrique de $K$ et $\mathfrak{C}$ le complété $p$-adique de $\bar{K}$. On note $\mathcal{O}_{K}$ (resp. $\mathcal{O}_{\bar{K}}$, resp. o) l'anneau de valuation de $K$ (resp. $\bar{K}$, resp. $\mathfrak{C})$. On pose $S=\operatorname{Spec}\left(\mathcal{O}_{K}\right)$ et on note $s(\operatorname{resp} . \eta)$ le point fermé (resp. générique) de $S$ et $\bar{\eta}$ le point géométrique de $S$ associé à $\bar{K}$.

Soit $X$ une $S$-courbe plate et propre à fibre générique lisse et géométriquement connexe, et $\bar{x}$ un point géométrique de $X_{\bar{\eta}}$. On dit qu'un fibré vectoriel $\mathcal{F}$ sur $X \otimes_{\mathcal{O}_{K}} \mathfrak{o}$ est de Deninger-Werner si l'image inverse de $\mathcal{F}$ sur la normalisation de chaque composante irréductible de $X_{s}$ est fortement semi-stable de degré 0 . À un tel fibré $\mathcal{F}$, Deninger et Werner associent une représentation du groupe fondamental étale $\pi_{1}\left(X_{\bar{\eta}}, \bar{x}\right)$ définie comme suit.

Pour tout entier $n \geqslant 1$, quitte à remplacer $K$ par une extension finie, il existe un morphisme propre $\varphi: X^{\prime} \rightarrow X$ tel que $\varphi_{\eta}$ soit étale fini, que $X^{\prime}$ soit une $S$-courbe semi-stable et que l'image réciproque de $\mathcal{F}_{n}=\mathcal{F} / p^{n} \mathcal{F}$ par la réduction modulo $p^{n}$ de $\varphi$ soit triviale (6.2). Le 'transport parallèle' permet alors de construire une représentation de $\pi_{1}\left(X_{\bar{\eta}}, \bar{x}\right)$ sur la fibre de $\mathcal{F}_{n}$ en $\bar{x}$ (cf. 6.9). Par passage à la limite projective, on obtient une $\mathfrak{o}$-représentation continue $p$-adique de $\pi_{1}\left(X_{\bar{\eta}}, \bar{x}\right)$.

1.3. Soit $C$ une $\bar{K}$-courbe propre, lisse et connexe, $\check{C}=C \otimes_{\bar{K}} \mathfrak{C}$ et $\bar{x}$ un point géométrique de $C$. On dit qu' un fibré vectoriel $F$ sur $\breve{C}$ est de Deninger-Werner si, quitte à remplacer $K$ par une extension finie, il existe un $S$-modèle propre et plat $X$ de $C$ et un fibré vectoriel de Deninger-Werner $\mathcal{F}$ sur $X \otimes_{\mathcal{O}_{K}} \mathfrak{o}$ de fibre générique $F$. On note $\mathfrak{V}_{\check{C}}^{\mathrm{DW}}$ la catégorie de tels fibrés et $\operatorname{Rep}_{\mathfrak{C}}^{\text {cont }}\left(\pi_{1}(C, \bar{x})\right)$ la catégorie des $\mathfrak{C}$-représentations continues $p$-adiques de $\pi_{1}(C, \bar{x})$ sur des $\mathfrak{C}$-espaces vectoriels de dimension finie (cf. 3.18). La construction de Deninger-Werner induit alors un 
foncteur (6.14.1)

$$
\mathbb{V}: \mathfrak{V}_{\check{C}}^{\mathrm{DW}} \rightarrow \operatorname{Rep}_{\mathfrak{C}}^{\text {cont }}\left(\pi_{1}(C, \bar{x})\right)
$$

Inspirés par le cas complexe, Deninger et Werner ont posé quelques questions concernant ce foncteur: est-il pleinement fidèle ? La cohomologie des systèmes locaux fournis par celui-ci admet-elle une filtration de Hodge-Tate? Leur construction est-elle compatible avec la correspondance de Simpson $p$-adique développée par Faltings [17] ? Le but de cet article est de répondre à ces questions.

1.4. Simultanément, Faltings a développé une correspondance pour les systèmes locaux $p$-adiques sur les variétés définies sur des corps $p$-adiques, inspiré par les travaux de Simpson dans le cas complexe. Rappelons que ce dernier a étendu le résultat de Narasimhan et Seshadri aux représentations linéaires quelconques du groupe fondamental topologique d'une variété complexe projective et lisse. Pour ce faire, on a besoin de la notion de fibré de Higgs : si $X$ est un schéma lisse de type fini sur un corps $F$, un fibré de Higgs sur $X$ est un couple $(M, \theta)$ formé d'un fibré vectoriel $M$ sur $X$ et d'un morphisme $\mathscr{O}_{X}$-linéaire $\theta: M \rightarrow M \otimes_{\mathscr{O}_{X}}$ $\Omega_{X / F}^{1}$ tel que $\theta \wedge \theta=0$ (cf. 2.10). Le résultat principal de Simpson [43] établit une équivalence de catégories entre la catégorie des représentations linéaires de dimension finie (à valeurs complexes) du groupe fondamental topologique d'une variété complexe projective et lisse $X$ et celle des fibrés de Higgs semi-stables de classes de Chern nulles sur $X$.

1.5. La construction de Faltings, appelée classiquement correspondance de Simpson $p$-adique, utilise sa théorie des extensions presque étales et prolonge ses travaux en théorie de Hodge $p$-adique, en particulier ceux qui concernent la décomposition de Hodge-Tate pour une variété propre et lisse sur un corps $p$-adique $[15,16]$. L'objet principal d'étude est la notion de représentation généralisée, qui étend celle de représentation $p$-adique du groupe fondamental géométrique. Ce sont, en termes simplifiés, des représentations semi-linéaires $p$-adiques continues du groupe fondamental géométrique dans des modules sur un certain anneau $p$-adique muni d'une action continue du groupe fondamental géométrique. Faltings construit un foncteur de la catégorie de ces représentations dans la catégorie des fibrés de Higgs [17]. Nous utiliserons la variante développée par Abbes et Gros [3], qui s'applique à une classe de représentations généralisées vérifiant une condition d'admissibilité à la Fontaine, dites de Dolbeault. Nous introduisons dans le même esprit une autre condition d'admissibilité, plus forte que celle de Dolbeault, que nous qualifions de Weil-Tate. Elle correspond aux fibrés de Higgs à champ de Higgs nul dans la correspondance de Simpson $p$ adique. 
Par ailleurs, en s'inspirant de la construction de Deninger-Werner, nous associons à certaines représentations généralisées, qualifiées de potentiellement libres de type fini, des représentations continues $p$-adiques du groupe fondamental géométrique. Les fibrés vectoriels de Deninger-Werner définissent naturellement des représentations généralisées potentiellement libres de type fini, et on retrouve ainsi le foncteur de Deninger-Werner. Les représentations généralisées de WeilTate sont aussi potentiellement libres de type fini. La principale question est de comparer ces deux sous-catégories. Notre principal résultat est que les représentations généralisées fournies par les fibrés vectoriels de Deninger-Werner sont de Weil-Tate. Ce résultat nous permet de répondre aux trois questions de Deninger et Werner.

1.6. Pour définir la notion de représentation généralisée, nous avons besoin du topos de Faltings. Soit $X$ un $S$-schéma de type fini à réduction semi-stable tel que $X_{\bar{\eta}}$ soit connexe. On désigne par $E$ la catégorie des morphismes de schémas $V \rightarrow U$ au-dessus du morphisme canonique $X_{\bar{\eta}} \rightarrow X$ tels que le morphisme $U \rightarrow X$ soit étale et que le morphisme $V \rightarrow U_{\bar{\eta}}$ soit fini étale (7.1). On équipe $E$ de la topologie co-évanescente engendrée par les recouvrements $\left\{\left(V_{i} \rightarrow U_{i}\right) \rightarrow\right.$ $(V \rightarrow U)\}_{i \in I}$ des deux types suivants :

(v) $U_{i}=U$ pour tout $i \in I$ et $\left(V_{i} \rightarrow V\right)_{i \in I}$ est un recouvrement.

(c) $\left(U_{i} \rightarrow U\right)_{i \in I}$ est un recouvrement et $V_{i}=U_{i} \times_{U} V$ pour tout $i \in I$.

Le site $E$ ainsi défini est appelé site de Faltings de $X$. On désigne par $\widetilde{E}$ et l'on appelle topos de Faltings de $X$ le topos des faisceaux d'ensembles sur $E$.

Pour tout schéma $Y$, on note $\mathbf{E}^{\prime} \mathbf{t}_{/ Y}$ (resp. $\mathbf{E}_{\mathbf{t}_{\mathrm{f} / Y}}$ ) la catégorie des schémas étales (resp. finis et étales) au-dessus de $Y$ munie de la topologie étale et $Y_{\text {ét }}$ (resp. $Y_{\text {fét }}$ ) le topos des faisceaux d'ensembles sur $\mathbf{E}_{/ Y}\left(\operatorname{resp} . \mathbf{E}_{\mathbf{f} / Y}\right)$. Les foncteurs

$$
\begin{aligned}
& \mathbf{E}_{\mathbf{t}_{\mathrm{f} / X_{\bar{\eta}}} \rightarrow E \quad V \mapsto(V \rightarrow X)} \\
& \mathbf{E}_{/ X} \rightarrow E \quad U \mapsto\left(U_{\bar{\eta}} \rightarrow U\right)
\end{aligned}
$$

sont continus et exacts à gauche. Ils définissent donc deux morphismes de topos

$$
\begin{aligned}
& \beta: \widetilde{E} \rightarrow X_{\bar{\eta}, \text { fét }}, \\
& \sigma: \widetilde{E} \rightarrow X_{\text {ét }} .
\end{aligned}
$$

Pour chaque objet $(V \rightarrow U)$ de $E$, on note $\bar{U}^{V}$ la clôture intégrale de $\bar{U}=$ $U \otimes_{\mathcal{O}_{K}} \mathcal{O}_{\bar{K}}$ dans $V$. On désigne par $\overline{\mathscr{B}}$ le préfaisceau d'anneaux sur $E$ défini pour tout $(V \rightarrow U) \in \mathbf{O b}(E)$ par

$$
\overline{\mathscr{B}}(V \rightarrow U)=\Gamma\left(\bar{U}^{V}, \mathscr{O}_{\bar{U}^{V}}\right) .
$$


En fait, celui-ci est un faisceau sur $E$ [4, III.8.16]. Les représentations généralisées sont essentiellement les $\overline{\mathscr{B}}$-modules de $\widetilde{E}$. Cependant, pour tenir compte de la topologie $p$-adique, nous travaillons avec le système projectif des anneaux $\overline{\mathscr{B}}_{n}=\overline{\mathscr{B}} / p^{n} \overline{\mathscr{B}}, n \geqslant 1$.

Pour tout entier $n \geqslant 1, \mathscr{B}_{n}$ est un objet de la fibre spéciale $\widetilde{E}_{s}$ de $\widetilde{E}$, c'est-à-dire du sous-topos fermé de $\widetilde{E}$ complémentaire de l'ouvert $\sigma^{*}\left(X_{\eta}\right)$ (7.10). Celui-ci s'insère dans un diagramme commutatif à isomorphisme canonique près

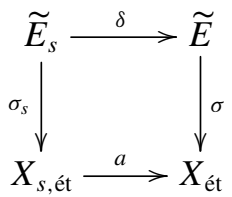

où $a$ est l'injection canonique, $\delta$ est le plongement canonique et $\sigma_{s}$ est induit $\operatorname{par} \sigma$.

Les systèmes projectifs d'objets de $\widetilde{E}_{s}$ indexés par l'ensemble ordonné des entiers naturels $\mathbb{N}$ forment un topos que l'on note $\widetilde{E}_{s}^{\mathbb{N}^{\circ}}$. On le munit de l'anneau $\breve{\mathscr{B}}=\left(\overline{\mathscr{B}}_{n}\right)_{n} \geqslant 1$ de $\widetilde{E}_{s}^{\mathbb{N}^{\circ}}$. On dit qu'un $\breve{\mathscr{B}}$-module $\left(M_{n}\right)_{n \geqslant 1}$ est adique si, pour tout entier $i \geqslant 1$, le morphisme $M_{i+1} \otimes_{\overline{\mathscr{B}}_{i+1}} \overline{\mathscr{B}}_{i} \rightarrow M_{i}$, déduit du morphisme de transition $M_{i+1} \rightarrow M_{i}$, est un isomorphisme.

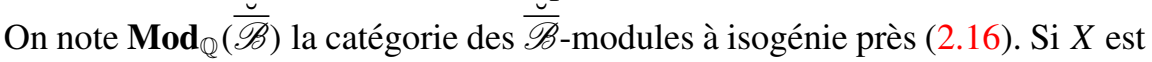
propre sur $S$, d'après le principal théorème de comparaison de Faltings (7.19) (cf. [16, Theorem 8], [3, 2.4.16]), l'image réciproque associée au morphisme $\beta$ induit un foncteur pleinement fidèle (8.8)

$$
\operatorname{Rep}_{\mathfrak{C}}^{\text {cont }}\left(\pi_{1}\left(X_{\bar{\eta}}, \bar{x}\right)\right) \rightarrow \operatorname{Mod}_{\mathbb{Q}}(\breve{\mathscr{B}})
$$

1.7. Supposons que $X$ soit une $S$-courbe semi-stable et posons $C=X_{\bar{\eta}}$. Soit $\bar{x}$ un point géométrique de $C$ et $n \geqslant 1$ un entier. Si $X^{\prime}$ est une $S$-courbe semi-stable, $\left(\widetilde{E}^{\prime}, \overline{\mathscr{B}}^{\prime}\right)$ le topos annelé de Faltings associé à $X^{\prime}$ et $\varphi: X^{\prime} \rightarrow X$ un $S$-morphisme propre, alors $\varphi$ induit par fonctorialité un morphisme de topos annelés $\Phi_{n}:\left(\widetilde{E}_{s}^{\prime}\right.$, $\left.\overline{\mathscr{B}}_{n}^{\prime}\right) \rightarrow\left(\widetilde{E}_{s}, \overline{\mathscr{B}}_{n}\right)$. On dit qu'un $\overline{\mathscr{B}}_{n}$-module $M_{n}$ est potentiellement libre de type fini s'il est de type fini et si, quitte à remplacer $K$ par une extension finie, il existe un $S$-morphisme propre $\varphi: X^{\prime} \rightarrow X$ tel que $X^{\prime}$ soit une $S$-courbe semi-stable, $\varphi_{\eta}$ soit fini étale et qu'avec les notations précédentes $\Phi_{n}^{*}\left(M_{n}\right)$ soit un $\overline{\mathscr{B}}_{n}^{\prime}$-module libre. Par 'transport parallèle', on associe à tout $\overline{\mathscr{B}}_{n}$-module potentiellement libre de type fini une représentation de $\pi_{1}(C, \bar{x})(8.10)$.

On dit qu'un $\breve{\mathscr{B}}$-module $M=\left(M_{n}\right)_{n \geqslant 1}$ est potentiellement libre de type fini s'il est adique de type fini et si, pour tout entier $n \geqslant 1, M_{n}$ est potentiellement libre de 


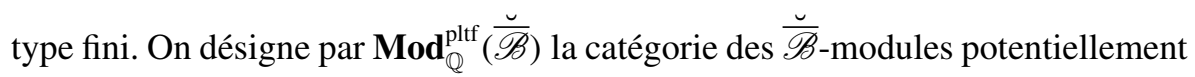
libres de type fini à isogénie près. La construction précédente induit un foncteur (8.14.1)

$$
\mathscr{W}: \operatorname{Mod}_{\mathbb{Q}}^{\mathrm{pltf}}(\breve{\mathscr{B}}) \rightarrow \operatorname{Rep}_{\mathfrak{C}}^{\mathrm{cont}}\left(\pi_{1}(C, \bar{x})\right)
$$

Celui-ci généralise le foncteur $\mathbb{V}(1.3 .1)$. En effet, notons Vect $_{\check{C}}$ la catégorie des fibrés vectoriels sur $\check{C}=C \otimes_{\bar{K}} \mathfrak{C}$. L'image réciproque associée au morphisme $\sigma$ induit un foncteur (cf. (5.14.2) et (7.12.4))

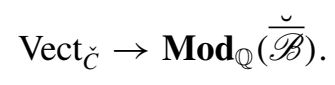

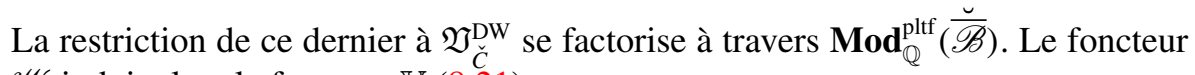
$\mathscr{W}$ induit alors le foncteur $\mathbb{V}(8.21)$.

Par ailleurs, le foncteur (1.6.5) se factorise à travers la sous-catégorie $\operatorname{Mod}_{\mathbb{Q}}^{\text {pltf }}(\breve{\mathscr{B}})$ de $\operatorname{Mod}_{\mathbb{Q}}(\breve{\mathscr{B}})$. Le composé de ce dernier et de $\mathscr{W}$ est isomorphe au foncteur identique (8.17(ii)).

On dit qu'un fibré vectoriel sur $\check{C}$ et une $\mathfrak{C}^{\mathfrak{C}}$-représentation continue $V$ de $\pi_{1}(C$, $\bar{x})$ sont $\overline{\mathscr{B}}_{\mathbb{Q}}$-associés si leurs images par les foncteurs (1.7.2) et (1.6.5) dans $\operatorname{Mod}_{\mathbb{Q}}(\breve{\mathscr{B}})$ sont isomorphes (cf. 10.2).

1.8. Soit $C$ une courbe propre et lisse sur $\bar{K}, \bar{x}$ un point géométrique de $C$ et $\check{C}=C \otimes_{\bar{K}} \mathfrak{C}$. On dit qu'un fibré vectoriel $F$ sur $\check{C}$ est de Weil-Tate si, quitte à remplacer $K$ par une extension finie, il existe un $S$-modèle semi-stable $X$ de $C$ et une $\mathfrak{C}$-représentation continue $V$ de $\pi_{1}(C, \bar{x})$ tels que $F$ et $V$ soient $\breve{\mathscr{B}}_{\mathbb{Q}^{-}}$ associés dans le topos annelé de Faltings relatif à $X$. On définit symétriquement

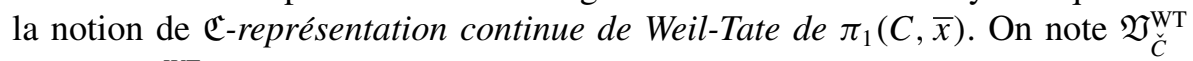
(resp. $\left.\operatorname{Rep}_{\mathfrak{C}}^{\mathrm{WT}}\left(\pi_{1}(C, \bar{x})\right)\right)$ la catégorie de tels fibrés (resp. telles $\mathfrak{C}$-représentations). On montre le résultat suivant.

THÉORÈME 1.9 (cf. 10.19). Il existe des équivalences de catégories quasi inverses l'une de l'autre

$$
\mathscr{V}: \mathfrak{V}_{\breve{C}}^{\mathrm{WT}} \rightarrow \operatorname{Rep}_{\mathfrak{C}}^{\mathrm{WT}}\left(\pi_{1}(C, \bar{x})\right) \quad \text { et } \quad \mathscr{T}: \operatorname{Rep}_{\mathfrak{C}}^{\mathrm{WT}}\left(\pi_{1}(C, \bar{x})\right) \rightarrow \mathfrak{V}_{\check{C}}^{\mathrm{WT}}
$$

Notre principal résultat est le suivant.

THÉORÈME 1.10 (cf. 14.5). Tout fibré vectoriel de Deninger-Werner sur $\check{C}$ est de Weil-Tate. 
La condition de Deninger-Werner peut se voir comme une condition d'admissibilité à la Fontaine. Elle présente toutefois des différences notables avec la condition d'admissibilité de Weil-Tate. D'une part, le fibré est trivialisé par une courbe semi-stable au-dessus de $X$ qui est finie et étale au-dessus de $C$, mais qui n'est a priori pas finie au dessus de $X$. D'autre part, on a besoin d'une infinité de tels revêtements, un pour chaque réduction modulo $p^{n}, n \geqslant 1$. La première difficulté peut être surmontée grâce à un résultat de Raynaud sur le quotient d'une courbe semi-stable par un groupe fini (5.11(iii), cf. aussi [38]). La seconde difficulté est plus sérieuse. On la surmonte grâce à la théorie des déformations des presque-modules dans le sens de Faltings (13.7).

PROPOSITION 1.11 (cf. 14.6). La restriction du foncteur $\mathscr{V} \grave{a} \mathfrak{V}_{\check{C}}^{\mathrm{DW}}$ s'identifie au foncteur $\mathbb{V}$.

On en déduit la pleine fidélité du foncteur de Deninger-Werner (1.3.1).

1.12. On démontre que pour qu'une $\mathfrak{C}$-représentation continue $V$ de $\pi_{1}(C, \bar{x})$ soit de Weil-Tate, il faut et il suffit que son image, par le foncteur (1.6.5), soit de Dolbeault et que le fibré de Higgs associé par la correspondance de Simpson $p$-adique soit muni du champ de Higgs nul (cf. 11.7). De plus, le fibré vectoriel sous-jacent à ce fibré de Higgs est donné par $\mathscr{T}(V)$ (1.9.1). Cela signifie que l'équivalence de catégories $\mathscr{T}$ (1.9.1) n'est autre que la correspondance de Simpson $p$-adique pour les fibrés de Higgs à champ de Higgs nul. Dans 11.8, on démontre l'existence d'une filtration de Hodge-Tate pour la cohomologie des représentations de Weil-Tate.

1.13. Il est utile de noter que Faltings mentionne dans le premier paragraphe de la section 5 de [17] que l'on peut généraliser la construction de Deninger et Werner pour certains fibrés de Higgs en comparant les déformations des vraies représentations et celles des représentations généralisées.

1.14. Après avoir fixé les notations générales dans la section 2, nous développons dans les sections 3 à 5 quelques préliminaires utiles pour la suite de cet article. La section 3 est consacrée à rappeler et étudier les presque-algèbres et les presquemodules développés par Faltings dans son approche de la théorie de Hodge $p$ adique. Pour alléger, nous avons choisi dans cet article de substituer au préfixe presque le préfixe $\alpha$ suggéré par le almost anglais. Dans la section 4, nous rappelons la fidélité du foncteur GAGA pour les modules cohérents sur un $\mathfrak{C}$ schéma de type fini. La section 5 contient des rappels et des compléments sur les courbes relatives. Dans la section 6, nous rappelons les résultats de Deninger et 
Werner. Suivant [4, III et VI], nous présentons le topos annelé de Faltings dans la section 7. Ensuite, nous rappelons le principal théorème de comparaison de Faltings et nous donnons quelques corollaires. Nous finissons cette section par la description du topos annelé de Faltings associé à un trait (7.26). Dans la section 8, nous présentons la construction du foncteur $\mathscr{W}(1.7 .1)$ et ses propriétés. Nous établissons un résultat de descente galoisienne pour le topos de Faltings dans la section 9. Grâce à ce résultat, nous démontrons une variante modulo $p^{n} \mathrm{du}$ théorème 1.10 ( $\mathrm{cf}$. 9.11). Dans la section 10, nous introduisons les notions de fibré vectoriel de Weil-Tate et de $\mathfrak{C}$-représentation continue de Weil-Tate. Nous construisons les foncteurs $\mathscr{V}$ et $\mathscr{T}(1.9 .1)$ et nous démontrons le théorème 1.9. Dans la section 11, nous comparons la correspondance de Simpson $p$-adique et la correspondance $\mathscr{T}$ (1.9.1). Dans la section 12, nous rappelons la notion de faisceaux de $\alpha$-modules suivant [3,1.4]. Nous développons ensuite une théorie de déformations des faisceaux de $\alpha$-modules suivant l'approche d'Illusie [27] dans la section 13. Nous étudions la relation entre les déformations des représentations du groupe fondamental et les déformations des $\alpha-\overline{\mathscr{B}}$-modules et nous démontrons le théorème 1.10 dans la section 14 .

\section{Notations et préliminaires}

2.1. Dans cet article, $p$ désigne un nombre premier, $K$ un corps de valuation discrète complet de caractéristique 0 dont le corps résiduel $k$ est une clôture algébrique de $\mathbb{F}_{p}$ et $\bar{K}$ une clôture algébrique de $K$. On note $\mathcal{O}_{K}$ l'anneau de valuation de $K$ et $\mathcal{O}_{\bar{K}}$ la clôture intégrale de $\mathcal{O}_{K}$ dans $\bar{K}$. On désigne par o le séparé complété $p$-adique de $\mathcal{O}_{\bar{K}}$, par $\mathfrak{C}$ son corps des fractions, par $\mathfrak{m}$ son idéal maximal et par $v$ la valuation de $\mathfrak{o}$ normalisée par $v(p)=1$. Pour tout entier $n \geqslant 1$, on pose $\mathfrak{o}_{n}=\mathcal{O}_{\bar{K}} / p^{n} \mathcal{O}_{\bar{K}}$.

On choisit un système compatible $\left(\gamma_{n}\right)$ de racines $n$-ièmes de $p$ dans $\mathcal{O}_{\bar{K}}$. Pour tout nombre rationnel $r>0$, on pose $p^{r}=\left(\gamma_{n}\right)^{r n}$ où $n$ est un entier $>0$ tel que $r n$ soit entier.

On pose $S=\operatorname{Spec}\left(\mathcal{O}_{K}\right), \bar{S}=\operatorname{Spec}\left(\mathcal{O}_{\bar{K}}\right), \stackrel{\check{S}}{S}=\operatorname{Spec}(\mathfrak{o}), \mathscr{S}=\operatorname{Spf}(\mathfrak{o})$ et pour tout entier $n \geqslant 1, S_{n}=\operatorname{Spec}\left(\mathcal{O}_{K} / p^{n} \mathcal{O}_{K}\right)$. On note $\eta$ (resp. $s$ ) le point générique (resp. fermé) de $S$ et $\bar{\eta}$ (resp. $\overline{\bar{\eta}}$ ) le point géométrique générique correspondant à $\bar{K}$ (resp. $\mathfrak{C}$ ). Pour tous $S$-schémas $X$ et $S^{\prime}$, on pose $X_{S^{\prime}}=X \times_{S} S^{\prime}$. Pour tout entier $n \geqslant 1$, on pose

$$
X_{n}=X \times{ }_{S} S_{n}, \quad \bar{X}=X \times{ }_{S} \bar{S}, \quad \stackrel{\check{X}}{X}=X \times{ }_{S} \stackrel{\check{\bar{S}}}{ }
$$

Pour tout morphisme de $S$-schémas $\pi: Y \rightarrow X$, on pose $\pi_{n}=\pi \times_{S} S_{n}$. Pour tout $\mathscr{O}_{X}$-module $M$ sur un $S$-schéma $X$, on note $M_{n}$ le $\mathscr{O}_{X_{n}}$-module $M \otimes_{\mathscr{O}_{S}} \mathscr{O}_{S_{n}}$ sur $X_{n}$. 
Si $T$ est un trait (i.e. le spectre d'un anneau de valuation discrète) et $\tau$ son point générique, on dit que $(T, \tau)$ est un trait génériquement pointé (ou simplement trait lorsqu'il n'y a aucun risque d'ambiguïté). Si $(T, \tau)$ et $\left(T^{\prime}, \tau^{\prime}\right)$ sont deux traits génériquement pointés, un morphisme $\left(T^{\prime}, \tau^{\prime}\right) \rightarrow(T, \tau)$ est un morphisme dominant $T^{\prime} \rightarrow T$. On dit qu'un tel morphisme est fini si le morphisme sousjacent $T^{\prime} \rightarrow T$ est fini.

2.2. On désigne par Ens la catégorie des ensembles que l'on considère ainsi comme un topos (ponctuel) et on le note aussi Pt [6, IV 2.2]. On désigne par Ab la catégorie des groupes abéliens.

2.3. Soit $\mathscr{A}$ une catégorie abélienne et $M, N$ deux objets de $\mathscr{A}$. On désigne par $\mathbf{D}(\mathscr{A})$ sa catégorie dérivée. On note abusivement $M$ le complexe concentré en degré 0 de valeur $M$. Pour tout entier $i$, on pose [47, 10.7.1]

$$
\operatorname{Ext}_{\mathscr{A}}^{i}(M, N)=\operatorname{Hom}_{\mathbf{D}(\mathscr{A})}(M, N[i]),
$$

où $[i]$ désigne le décalage de degré $i$.

Soit $F: \mathscr{A} \rightarrow \mathscr{A}^{\prime}$ un foncteur exact entre catégories abéliennes. Il s'étend en un foncteur de $\mathbf{D}(\mathscr{A})$ dans $\mathbf{D}\left(\mathscr{A}^{\prime}\right)$. En vertu de (2.3.1), il induit, pour tous objets $M$ et $N$ de $\mathscr{A}$ et tout entier $i$, un morphisme canonique

$$
\operatorname{Ext}_{\mathscr{A}}^{i}(M, N) \rightarrow \operatorname{Ext}_{\mathscr{A}^{\prime}}^{i}(F(M), F(N)) .
$$

Pour tous objets $M$ et $N$ de $\mathscr{A}$, le groupe $\operatorname{Ext}_{\mathscr{A}}^{1}(M, N)$ classifie les extensions de $M$ par $N$ dans $\mathscr{A}$ (cf. [48, 13.27.6]).

2.4. Soit $\mathscr{A}$ une catégorie abélienne ayant suffisamment d'injectifs et $M$ un objet de $\mathscr{A}$. Le foncteur $\operatorname{Hom}_{\mathscr{A}}(M,-): \mathscr{A} \rightarrow \mathbf{A b}$ est exact à gauche. On désigne par

$$
\operatorname{Ext}_{\mathscr{A}}^{i}(M,-): \mathscr{A} \rightarrow \mathbf{A b} \quad \forall i \geqslant 0
$$

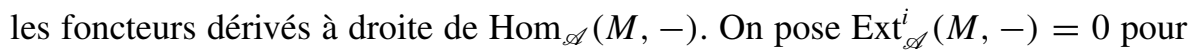
$i<0$. Cette définition est compatible avec (2.3.1) (cf. [47, 10.7.4 et 10.7.5]).

2.5. Soit $\mathscr{A}$ une catégorie abélienne et $\mathrm{E}$ une suite spectrale dans $\mathscr{A}$

$$
\mathrm{E}_{2}^{i, j} \Rightarrow \mathrm{E}^{i+j}
$$

telle que $\mathrm{E}_{2}^{i, j}=0$ si $i<0$ ou $j<0$. Les termes de bas degré fournissent une suite exacte [33, Appendix B]

$$
0 \rightarrow \mathrm{E}_{2}^{1,0} \rightarrow \mathrm{E}^{1} \rightarrow \mathrm{E}_{2}^{0,1} \rightarrow \mathrm{E}_{2}^{2,0} \rightarrow \mathrm{E}_{1}^{2} \rightarrow \mathrm{E}_{2}^{1,1},
$$


où $\mathrm{E}_{1}^{2}=\operatorname{Ker}\left(\mathrm{E}^{2} \rightarrow \mathrm{E}_{2}^{0,2}\right)$. Soit $\mathrm{E}^{\prime}$ une autre suite spectrale

$$
\mathrm{E}_{2}^{\prime i, j} \Rightarrow \mathrm{E}^{\prime i+j}
$$

telle que $\mathrm{E}_{2}^{i, j}=0$ si $i<0$ ou $j<0$ et $u: \mathrm{E} \rightarrow \mathrm{E}^{\prime}$ un morphisme de suites spectrales [24, 0.11.1.2]. On a alors un diagramme commutatif

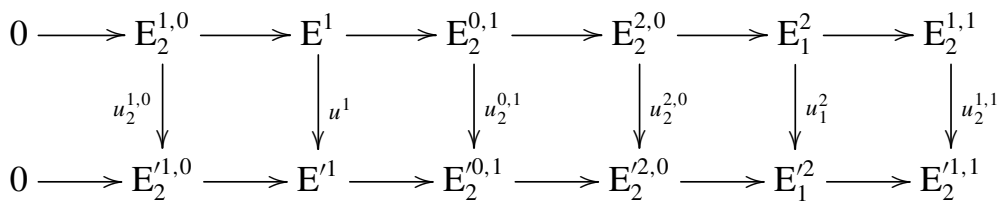

LEMME 2.6. Conservons les notations de 2.5 et supposons que les morphismes $u_{2}^{i, j}$ sont des isomorphismes pour $i+j \leqslant 1$ et sont des monomorphismes pour $i+j=2$. Alors, le morphisme $u^{n}: \mathrm{E}^{n} \rightarrow \mathrm{E}^{\prime n}$ est un isomorphisme si $n=0,1$ et un monomorphisme si $n=2$.

Preuve. Il est clair que $u^{0}$ est un isomorphisme. D'après (2.5.3), le lemme des cinq et le lemme du serpent, on déduit que le morphisme $u^{1}$ est un isomorphisme et que le morphisme $u_{1}^{2}$ est un monomorphisme. Par suite, on en déduit un diagramme commutatif

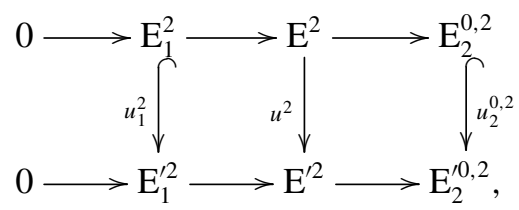

d'où l'injectivité de $u^{2}$.

2.7. Pour tout schéma $X$, on note $\operatorname{Pic}(X)=\mathrm{H}^{1}\left(X, \mathscr{O}_{X}^{\times}\right)$le groupe des classes d'isomorphismes de $\mathscr{O}_{X}$-modules inversibles. Soit $f: X \rightarrow T$ un morphisme propre de schémas. On désigne par $\mathrm{Pic}_{X / T}$ le foncteur de Picard relatif de $X$ audessus de $T$, défini pour tout $T$-schéma $T^{\prime}$ par

$$
\operatorname{Pic}_{X / T}\left(T^{\prime}\right)=\mathrm{H}^{0}\left(T^{\prime}, \mathrm{R}_{\mathrm{fppf}}^{1} f_{*}^{\prime}\left(\mathbb{G}_{m}\right)\right),
$$

où $f^{\prime}: X \times_{T} T^{\prime} \rightarrow T^{\prime}$ est la projection canonique et $\mathbb{G}_{m}$ est le faisceau fppf qui à chaque schéma $Y$ associe le groupe $\Gamma\left(Y, \mathscr{O}_{Y}^{*}\right)$ (cf. [37, 1.2]). Soit $t$ un point de $T$. D'après (2.7.1), on a $\operatorname{Pic}_{X / T}(t)=\operatorname{Pic}_{X_{t} / t}(t)$. Le foncteur $\mathrm{Pic}_{X_{t} / t}$ est représentable par un schéma en groupes localement de type fini (cf. [37, 1.5.2]). Il possède un 
plus petit sous-groupe ouvert connexe, sa composante neutre $\mathrm{Pic}_{X_{t} / t}^{0}\left[11, \mathrm{VI}_{\mathrm{A}}, 2\right]$. On définit le sous-foncteur $\operatorname{Pic}_{X / T}^{0}$ de $\operatorname{Pic}_{X / T}$ comme suit : pour tout $T$-schéma $T^{\prime}$, $\operatorname{Pic}_{X / T}^{0}\left(T^{\prime}\right)$ est le sous-groupe de $\operatorname{Pic}_{X / T}\left(T^{\prime}\right)$ formé des éléments qui pour chaque point $t$ de $T^{\prime}$ induisent par fonctorialité un élément de $\operatorname{Pic}_{X_{t} / t}^{0}(t)$.

2.8. Soit $X$ un schéma. On dit qu'un $\mathscr{O}_{X}$-module quasi cohérent $F$ est un fibré vectoriel sur $X$ s'il est localement libre de type fini. On désigne par Vect ${ }_{X}$ la souscatégorie pleine de la catégorie des $\mathscr{O}_{X}$-modules formée des fibrés vectoriels.

2.9. Soit $(\mathcal{T}, A)$ un topos annelé. On désigne $\operatorname{par} \operatorname{Mod}(\mathcal{T}, A)$ la catégorie des $A$ modules de $\mathcal{T}$. On dit qu'un $A$-module $M$ de $\mathcal{T}$ est localement projectif de type fini si les conditions équivalentes suivantes sont satisfaites (cf. [4, III.2.8]) :

(i) $M$ est de type fini et le foncteur $\mathscr{H} o m_{A}(M, \cdot)$ est exact;

(ii) $M$ est de type fini et tout épimorphisme de $A$-modules $N \rightarrow M$ admet localement une section;

(iii) $M$ est localement facteur direct d'un $A$-module libre de type fini.

On désigne par $\mathbf{L} \mathbf{L}^{\mathrm{tf}}(\mathcal{T}, A)$ (resp. $\left.\mathbf{L} \mathbf{P}^{\mathrm{tf}}(\mathcal{T}, A)\right)$ la sous-catégorie pleine de $\operatorname{Mod}(\mathcal{T}, A)$ formée des $A$-modules localement libres (resp. localement projectifs) de type fini de $\mathcal{T}$.

Un $A$-module localement projectif de type fini est plat en vertu de (iii). Soit $M^{\prime}$ et $M^{\prime \prime}$ deux $A$-modules localement projectifs de type fini. D'après (iii), le $A$-module $\mathscr{H}_{0} m_{A}\left(M^{\prime}, M^{\prime \prime}\right)$ est aussi localement projectif de type fini. Soit $0 \rightarrow M^{\prime} \rightarrow M \rightarrow M^{\prime \prime} \rightarrow 0$ une suite exacte de $\operatorname{Mod}(\mathcal{T}, A)$. On en déduit que, pour tout $i \geqslant 1$, le foncteur $\mathscr{E} x t_{A}^{i}(M,-)$ est nul. Le module $M$ est donc aussi localement projectif de type fini.

Étant donné un $A$-module localement projectif de type fini $M$, la suite spectrale qui relie les Ext locaux et globaux [6, V 6.1] induit, pour tout $A$-module $N$ et tout entier $i \geqslant 0$, un isomorphisme

$$
\operatorname{Ext}_{A}^{i}(M, N) \stackrel{\sim}{\rightarrow} \mathrm{H}^{i}\left(\mathcal{T}, \mathscr{H} o m_{A}(M, N)\right)
$$

2.10. Soit $(\mathcal{T}, A)$ un topos annelé et $E$ un $A$-module de $\mathcal{T}$. Un $A$-module de Higgs à coefficients dans $E$ est un couple $(M, \theta)$ formé d'un $A$-module $M$ de $\mathcal{T}$ et d'un morphisme $A$-linéaire $\theta: M \rightarrow M \otimes_{A} E$ tel que $\theta \wedge \theta=0$.

2.11. Soit $\mathcal{T}$ un topos. Les systèmes projectifs d'objets de $\mathcal{T}$ indexés par l'ensemble ordonné des entiers naturels $\mathbb{N}$ forment un topos que l'on note $\mathcal{T}^{\mathbb{N}^{\circ}}$ 
(cf. [4, VI.7.1]). Le foncteur

$$
\lambda^{*}: \mathcal{T} \rightarrow \mathcal{T}^{\mathbb{N}^{\circ}}
$$

qui à un objet $F$ associe le foncteur constant $\mathbb{N}^{\circ} \rightarrow \mathcal{T}$ de valeur $F$ est exact à gauche. Il admet pour adjoint à droite le foncteur

$$
\lambda_{*}: \mathcal{T}^{\mathbb{N}^{\circ}} \rightarrow \mathcal{T}
$$

qui à un foncteur $\mathbb{N}^{\circ} \rightarrow \mathcal{T}$ associe sa limite projective [4, III.7.4]. Le couple $\left(\lambda_{*}, \lambda^{*}\right)$ définit donc un morphisme de topos

$$
\lambda: \mathcal{T}^{\mathbb{N}^{\circ}} \rightarrow \mathcal{T}
$$

Étant donné un anneau $\breve{A}=\left(A_{n}\right)_{n \geqslant 1}$ de $\mathcal{T}^{\mathbb{N}^{\circ}}$, on dit qu'un $\breve{A}$-module $M=$ $\left(M_{n}\right)_{n \geqslant 1}$ de $\mathcal{T}^{\mathbb{N}^{\circ}}$ est adique si pour tous entiers $i$ et $j$ tels que $1 \leqslant i \leqslant j$, le morphisme $M_{j} \otimes_{A_{j}} A_{i} \rightarrow M_{i}$ déduit du morphisme de transition $M_{j} \rightarrow M_{i}$ est un isomorphisme.

2.12. Étant donné un morphisme de topos $\gamma: \mathcal{T}_{1} \rightarrow \mathcal{T}_{3}$ et un groupe abélien $\mathscr{F}$ de $\mathcal{T}_{3}$, on définit, pour tout entier $i \geqslant 0$, un morphisme

$$
\gamma^{*}: \mathrm{H}^{i}\left(\mathcal{T}_{3}, \mathscr{F}\right) \rightarrow \mathrm{H}^{i}\left(\mathcal{T}_{1}, \gamma^{*}(\mathscr{F})\right)
$$

comme le composé

$$
\mathrm{H}^{i}\left(\mathcal{T}_{3}, \mathscr{F}\right) \rightarrow \mathrm{H}^{i}\left(\mathcal{T}_{3}, \gamma_{*} \gamma^{*}(\mathscr{F})\right) \rightarrow \mathrm{H}^{i}\left(\mathcal{T}_{1}, \gamma^{*}(\mathscr{F})\right),
$$

où la première flèche est induite par le morphisme d'adjonction id $\rightarrow \gamma_{*} \gamma^{*}$ et la seconde flèche est induite par la suite spectrale de Cartan-Leray.

Supposons que $\gamma$ soit le composé de deux morphismes de topos $\mathcal{T}_{1} \stackrel{\alpha}{\rightarrow} \mathcal{T}_{2} \stackrel{\beta}{\rightarrow} \mathcal{T}_{3}$. Alors, pour tout entier $i \geqslant 0$ et tout faisceau abélien $\mathscr{F}$ de $\mathcal{T}_{3}$, le morphisme composé

$$
\mathrm{H}^{i}\left(\mathcal{T}_{3}, \mathscr{F}\right) \stackrel{\beta^{*}}{\rightarrow} \mathrm{H}^{i}\left(\mathcal{T}_{2}, \beta^{*}(\mathscr{F})\right) \rightarrow \mathrm{H}^{i}\left(\mathcal{T}_{2}, \alpha_{*} \gamma^{*}(\mathscr{F})\right) \rightarrow \mathrm{H}^{i}\left(\mathcal{T}_{1}, \gamma^{*}(\mathscr{F})\right),
$$

où la deuxième flèche est induite par le morphisme d'adjonction $\beta^{*} \rightarrow \alpha_{*} \alpha^{*} \beta^{*}$ et la troisième flèche est induite par la suite spectrale de Cartan-Leray, s'identifie au morphisme (2.12.1). En effet, si l'on note, pour $i=1,2,3, \Gamma_{i}: \mathcal{T}_{i} \rightarrow \mathbf{A b}$ le foncteur 'sections globales', le morphisme composé (2.12.3) est induit par le morphisme composé de la catégorie dérivée $\mathbf{D}(\mathbf{A b})$ :

$$
\mathrm{R} \Gamma_{3} \mathscr{F} \rightarrow \mathrm{R} \Gamma_{3} \mathrm{R} \beta_{*} \beta^{*} \mathscr{F} \rightarrow \mathrm{R} \Gamma_{2} \mathrm{R} \alpha_{*} \alpha^{*} \beta^{*} \mathscr{F}=\mathrm{R} \Gamma_{1} \alpha^{*} \beta^{*} \mathscr{F} .
$$


Celui-ci s'identifie au morphisme induit par adjonction

$$
\mathrm{R} \Gamma_{3} \mathscr{F} \rightarrow \mathrm{R} \Gamma_{3} \mathrm{R} \gamma_{*} \gamma^{*} \mathscr{F}=\mathrm{R} \Gamma_{1} \alpha^{*} \beta^{*} \mathscr{F},
$$

qui fournit le morphisme (2.12.1).

2.13. Pour tout schéma $X$, on note $\mathbf{E} \mathbf{t}_{/ X}$ (resp. $X_{\text {ét }}$ ) le site (resp. topos) étale de $X$. On désigne par $\mathbf{E}_{\mathrm{coh} / X}\left(\operatorname{resp} . \mathbf{E ́ t}_{\mathrm{scoh} / X}\right)$ la sous-catégorie pleine de $\mathbf{E ́ t}_{/ X}$ formée des schémas étales de présentation finie sur $X$ (resp. étales, séparés et de présentation finie sur $X$ ), munie de la topologie induite par celle de $\mathbf{E}_{/ X}$. Si $X$ est quasi séparé, le foncteur de restriction de $X_{\text {ét }}$ dans le topos des faisceaux d'ensembles sur $\mathbf{E}_{\text {coh } / X}$ (resp. $\mathbf{E}_{\text {scoh } / X}$ ) est une équivalence de catégories [6, VII 3.1 et 3.2].

On désigne par $X_{\text {zar }}$ le topos de Zariski de $X$ et par

$$
u_{X}: X_{\text {ét }} \rightarrow X_{\text {zar }}
$$

le morphisme canonique [6, VII 4.2.2]. Si $F$ est un $\mathscr{O}_{X}$-module quasi cohérent de $X_{\text {zar }}$, on note encore $F$ le faisceau de $X_{\text {ét }}$ défini pour tout $X$-schéma étale $X^{\prime}$ par [6, VII 2 c], cf. aussi [4, III.2.9]

$$
F\left(X^{\prime}\right)=\Gamma\left(X^{\prime}, F \otimes_{\mathscr{O}_{X}} \mathscr{O}_{X^{\prime}}\right) .
$$

On désigne par $\mathbf{E} \mathbf{t}_{\mathrm{f} / X}$ le site fini étale de $X$, c'est-à-dire la sous-catégorie pleine de $\mathbf{E} \mathbf{t}_{/ X}$ formée des schémas étales et finis sur $X$, munie de la topologie induite par celle de $\mathbf{E} \mathbf{t}_{/ X}$. On note $X_{\text {fét }}$ le topos fini étale de $X$, c'est-à-dire le topos des faisceaux d'ensembles sur $\mathbf{E} \mathbf{t}_{\mathrm{f} / X}$. Le foncteur d'injection canonique $\mathbf{E}_{\mathrm{f} / X} \rightarrow \mathbf{E}^{\prime} \mathbf{t}_{/ X}$ est continu. Il induit donc un morphisme de topos que l'on note

$$
\rho_{X}: X_{\text {ét }} \rightarrow X_{\text {fét }} \text {. }
$$

2.14. Soit $X$ un schéma connexe, $\bar{x}$ un point géométrique de $X$. On désigne par $\pi_{1}(X, \bar{x})$ le groupe fondamental de $X$ en $\bar{x}$ et par $\mathbf{B}_{\pi_{1}(X, \bar{x})}$ le topos classifiant du groupe profini $\pi_{1}(X, \bar{x})$, c'est-à-dire la catégorie des ensembles discrets munis d'une action continue à gauche de $\pi_{1}(X, \bar{x})$ [6, IV 2.7]. Le foncteur fibre en $\bar{x}$

$$
\omega_{\bar{x}}: \mathbf{E}_{\mathrm{f} / X} \rightarrow \mathbf{B}_{\pi_{1}(X, \bar{x})},
$$

qui à tout revêtement étale $Y$ de $X$ associe l'ensemble des points géométriques de $Y$ au-dessus de $\bar{x}$, induit une équivalence de catégories [4, VI.9.8]

$$
\mu_{\bar{x}}: \mathbf{B}_{\pi_{1}(X, \bar{x})} \stackrel{\sim}{\rightarrow} X_{\text {fét }} .
$$


Le foncteur $\omega_{\bar{x}}$ est pro-représentable par un système projectif $\left(X_{i}, \bar{x}_{i}, \phi_{i j}\right)_{i \in I}$ de revêtements étales galoisiens pointés de $X$, indexé par un ensemble ordonné filtrant $I$, où $\bar{x}_{i}$ est un point géométrique de $X_{i}$ au-dessus de $\bar{x}$ et $\phi_{i j}: X_{i} \rightarrow X_{j}$ $(i \geqslant j)$ est un épimorphisme qui envoie $\bar{x}_{i}$ sur $\bar{x}_{j}$. Il est appelé pro-objet universel galoisien de $X$ en $\bar{x}$. Le foncteur

$$
\nu_{\bar{x}}: X_{\text {fét }} \rightarrow \mathbf{B}_{\pi_{1}(X, \bar{x})} \quad F \mapsto \underset{i \in I}{\lim } F\left(X_{i}\right)
$$

est une équivalence de catégories, quasi inverse de $\mu_{\bar{x}}$ [4, VI.9.8].

LEMME 2.15. Soit $X$ un schéma quasi compact, quasi séparé et connexe et $\mathbb{L}$ un faisceau abélien de torsion de $X_{\text {fét. Le morphisme canonique }}$

$$
\rho_{X}^{*}: \mathrm{H}^{i}\left(X_{\text {fét }}, \mathbb{L}\right) \rightarrow \mathrm{H}^{i}\left(X_{\text {ét }}, \rho_{X}^{*}(\mathbb{L})\right)
$$

est un isomorphisme si $i=0,1$ et un monomorphisme si $i=2$.

Preuve. Le morphisme d'adjonction id $\rightarrow \rho_{X *} \rho_{X}^{*}$ est un isomorphisme [4, VI.9.18], d'où la proposition pour $i=0$. Démontrons-la pour $i=1$. Les topos $X_{\text {ét }}$ et $X_{\text {fét }}$ sont cohérents [4, VI.9.12]. Par [4, VI.9.20] et [6, VI 5.1], on peut se borner au cas où $\rho_{X}^{*}(\mathbb{L})$ est un faisceau abélien localement constant et constructible, i.e. représentable par un schéma en groupe fini et étale $G$ sur $X$. Le faisceau $\mathrm{R}^{1} \rho_{X *}\left(\rho_{X}^{*}(\mathbb{L})\right)$ de $X_{\text {fét }}$ est le faisceau sur $\mathbf{E}_{\mathbf{f} / X}$ associé au préfaisceau défini par $Y \mapsto \mathrm{H}^{1}\left(Y_{\text {ét }}, \rho_{X}^{*}(\mathbb{L})\right)$. La catégorie des $\rho_{X}^{*}(\mathbb{L})$-torseurs sur $Y_{\text {ét }}$ est équivalente à la catégorie des $G_{Y}$-fibrés principaux homogènes sur $Y$ pour la topologie étale, où $G_{Y}=G \times_{X} Y\left[6\right.$, VII 2 b)]. Tout $G_{Y}$-fibré principal homogène sur $Y$ est trivialisé par un revêtement étale de $Y$. Le faisceau $\mathrm{R}^{1} \rho_{X *}\left(\rho_{X}^{*}(\mathbb{L})\right)$ est donc trivial.

On a une suite spectrale

$$
\mathrm{H}^{i}\left(X_{\text {fét }}, \mathrm{R}^{j} \rho_{X *}\left(\rho_{X}^{*}(\mathbb{L})\right)\right) \Rightarrow \mathrm{H}^{i+j}\left(X_{\text {ét }}, \rho_{X}^{*}(\mathbb{L})\right) .
$$

On en déduit un isomorphisme

$$
\mathrm{H}^{1}\left(X_{\text {fét }}, \rho_{X *} \rho_{X}^{*}(\mathbb{L})\right) \stackrel{\sim}{\rightarrow} \mathrm{H}^{1}\left(X_{\text {ét }}, \rho_{X}^{*}(\mathbb{L})\right) .
$$

La proposition pour $i=1$ résulte de ce dernier et de l'isomorphisme id $\stackrel{\sim}{\rightarrow} \rho_{X *} \rho_{X}^{*}$. Comme $\mathrm{R}^{1} \rho_{X *}\left(\rho_{X}^{*}(\mathbb{L})\right)$ est nul, on en déduit par (2.5.2) une suite exacte

$$
0 \rightarrow \mathrm{H}^{2}\left(X_{\text {fét }}, \rho_{X *}\left(\rho_{X}^{*}(\mathbb{L})\right)\right) \rightarrow \mathrm{H}^{2}\left(X_{\text {ét }}, \rho_{X}^{*}(\mathbb{L})\right) \rightarrow \mathrm{H}^{0}\left(X_{\text {fét }}, \mathrm{R}^{2} \rho_{X *}\left(\rho_{X}^{*}(\mathbb{L})\right)\right) .
$$

La proposition pour $i=2$ résulte alors de ce dernier et de l'isomorphisme $\mathrm{id} \stackrel{\sim}{\rightarrow} \rho_{X *} \rho_{X}^{*}$. 
2.16. Si $\mathscr{C}$ est une catégorie additive, on désigne par $\mathscr{C}_{\mathbb{Q}}$ et l'on appelle catégorie des objets de $\mathscr{C}$ à isogénie près [4, III.6.1] la catégorie ayant mêmes objets que $\mathscr{C}$ et telle que l'ensemble des morphismes soit donné, pour tous $M$, $N \in \mathbf{O b}(\mathscr{C})$, par

$$
\operatorname{Hom}_{\mathscr{C} \mathbb{Q}}(M, N)=\operatorname{Hom}_{\mathscr{C}}(M, N) \otimes_{\mathbb{Z}} \mathbb{Q} .
$$

On désigne par

$$
\mathscr{C} \rightarrow \mathscr{C}_{\mathbb{Q}}, \quad M \mapsto M_{\mathbb{Q}}
$$

le foncteur canonique. Deux objets de $\mathscr{C}$ sont dits isogènes s'ils sont isomorphes dans la catégorie $\mathscr{C}_{\mathbb{Q}}$. Si $\mathscr{C}$ est une catégorie abélienne, la catégorie $\mathscr{C}_{\mathbb{Q}}$ est abélienne et le foncteur canonique (2.16.2) est exact [4, III.6.1.4]. Tout foncteur additif (resp. exact) entre catégories additives (resp. abéliennes) $\mathscr{C} \rightarrow \mathscr{C}^{\prime}$ induit un foncteur additif (resp. exact) $\mathscr{C}_{\mathbb{Q}} \rightarrow \mathscr{C}_{\mathbb{Q}}^{\prime}$ compatible aux foncteurs canoniques (2.16.2) [4, III.6.1.5].

La famille des isogénies de $\mathscr{C}$ permet un calcul de fractions bilatère [27, I 1.4.2]. La catégorie $\mathscr{C}_{\mathbb{Q}} \mathrm{s}^{\prime}$ identifie à la catégorie localisée par rapport aux isogénies et le foncteur (2.16.2) est le foncteur de localisation (cf. [4, III.6.1]).

2.17. Soit $(\mathcal{T}, A)$ un topos annelé. On désigne par $\operatorname{Mod}_{\mathbb{Q}}(\mathcal{T}, A)$ la catégorie des $A$-modules de $\mathcal{T}$ à isogénie près (2.16) dont les objets sont appelés les $A_{\mathbb{Q}}$-modules. Étant donné deux $A_{\mathbb{Q}}$-modules $M$ et $N$, on note $\operatorname{Hom}_{A_{\mathbb{Q}}}(M, N)$ le groupe des morphismes de $M$ dans $N$ dans $\operatorname{Mod}_{\mathbb{Q}}(\mathcal{T}, A)$. Rappelons que la catégorie $\operatorname{Mod}_{\mathbb{Q}}(\mathcal{T}, A)$ a suffisamment d'injectifs [4, III.6.1.6] et reprenons les notations de (2.4) pour cette catégorie. Pour tout entier $i \geqslant 0$ et tous $A_{\mathbb{Q}^{-}}$ modules $M$ et $N$, on pose $\operatorname{Ext}_{A_{\mathbb{Q}}}^{i}(M, N)=\operatorname{Ext}_{\operatorname{Mod}_{\mathbb{Q}}(\mathcal{T}, A)}^{i}(M, N)$ qui est un $\mathbb{Q}$ espace vectoriel. En particulier, pour tout entier $i \geqslant 0$ et tout $A_{\mathbb{Q}^{-}}$-module $N$, on pose

$$
\mathrm{H}^{i}(\mathcal{T}, N)=\operatorname{Ext}_{A_{\mathbb{Q}}}^{i}\left(A_{\mathbb{Q}}, N\right)
$$

On note $\operatorname{Vect}_{\mathbb{Q}}$ la catégorie des $\mathbb{Q}$-espaces vectoriels. Le foncteur canonique $\mathbf{A b} \rightarrow$ Vect $_{\mathbb{Q}}$ induit une équivalence de catégories $\mathbf{A} \mathbf{b}_{\mathbb{Q}} \stackrel{\sim}{\rightarrow}$ Vect $_{\mathbb{Q}}$. Étant donné un $A$-module $M$ de $\mathcal{T}$, on a un diagramme commutatif

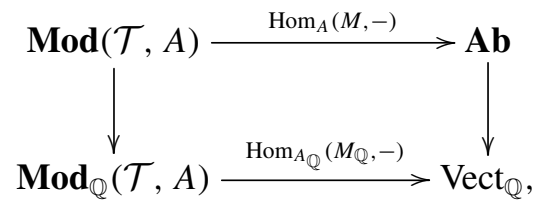

où les flèches verticales sont les foncteurs canoniques. Ceux-ci sont exacts et transforment les objets injectifs en des objets injectifs [4, III.6.1.6]. Pour tout 
entier $i \geqslant 0$ et tous $A$-modules $M$ et $N$, on en déduit par la suite spectrale de Cartan-Leray un isomorphisme

$$
\operatorname{Ext}_{A}^{i}(M, N) \otimes_{\mathbb{Z}} \mathbb{Q} \stackrel{\sim}{\rightarrow} \operatorname{Ext}_{A_{\mathbb{Q}}}^{i}\left(M_{\mathbb{Q}}, N_{\mathbb{Q}}\right) .
$$

En particulier, pour tout entier $i \geqslant 0$ et tout $A$-module $N$, on a un isomorphisme

$$
\mathrm{H}^{i}(\mathcal{T}, N) \otimes_{\mathbb{Z}} \mathbb{Q} \simeq \mathrm{H}^{i}\left(\mathcal{T}, N_{\mathbb{Q}}\right)
$$

2.18. Soit $\mathscr{A}$ une catégorie abélienne, End(id $\mathscr{A})$ l'anneau des endomorphismes du foncteur identique de $\mathscr{A}$ et $\varphi: \mathfrak{o} \rightarrow \operatorname{End}\left(\mathrm{id}_{\mathscr{A}}\right)$ un homomorphisme. Pour tout objet $M$ de $\mathscr{A}$ et tout $\gamma \in \mathfrak{o}$, on note $\mu_{\gamma}(M)$ l'endomorphisme de $M$ défini par $\varphi(\gamma)$. En particulier, pour tous objets $M$ et $N$ de $\mathscr{A}, \operatorname{Hom}_{\mathscr{A}}(M, N)$ est muni d'une structure de $\mathfrak{o}$-module.

On dit qu'un objet $M$ de $\mathscr{A}$ est $\alpha$-nul s'il est annulé par tout élément de $\mathfrak{m}$, i.e. si $\mu_{\gamma}(M)=0$ pour tout $\gamma \in \mathfrak{m}$. Pour toute suite exacte $0 \rightarrow M^{\prime \prime} \rightarrow M \rightarrow M^{\prime} \rightarrow 0$, pour que $M$ soit $\alpha$-nul, il faut et il suffit que $M^{\prime \prime}$ et $M^{\prime}$ soient $\alpha$-nuls. On appelle catégorie des $\alpha$-objets de $\mathscr{A}$ et l'on note $\alpha-\mathscr{A}$ le quotient de la catégorie $\mathscr{A}$ par la sous-catégorie épaisse formée des objets $\alpha$-nuls ([20, III, Section 1]; cf. aussi $[3,1.4 .2])$. On note

$$
\alpha: \mathscr{A} \rightarrow \alpha-\mathscr{A}, \quad M \mapsto \alpha(M)
$$

le foncteur canonique; on notera aussi $M^{\alpha}$ au lieu de $\alpha(M)$ lorsqu'il n'y a aucun risque de confusion. La catégorie $\alpha-\mathscr{A}$ est abélienne et le foncteur $\alpha$ est exact [20, III, Section 1, prop. 1]. On dit qu'un morphisme $f$ de $\mathscr{A}$ est un $\alpha$ isomorphisme (resp. $\alpha$-monomorphisme, resp. $\alpha$-épimorphisme) si $\alpha(f)$ est un isomorphisme (resp. monomorphisme, resp. épimorphisme), autrement dit si son noyau et son conoyau (resp. son noyau, resp. son conoyau) sont $\alpha$-nuls.

La famille des $\alpha$-isomorphismes de $\mathscr{A}$ permet un calcul de fractions bilatère [27, I 1.4.2]. La catégorie $\alpha-\mathscr{A}$ s'identifie à la catégorie localisée par rapport aux $\alpha$-isomorphismes et $\alpha$ (2.18.1) est le foncteur canonique (de localisation).

LEMME 2.19 [3, 1.4.3]. Les hypothèses étant celles de 2.18, soit, de plus, $f: M \rightarrow N$ un morphisme de $\mathscr{A}$ et $\gamma \in \mathfrak{o}$ tels que le noyau et le conoyau de $f$ soient annulés par $\gamma$. Alors, il existe un morphisme $g: N \rightarrow M$ de $\mathscr{A}$ tel que $g \circ f=\mu_{\gamma^{2}}(M)$ et $f \circ g=\mu_{\gamma^{2}}(N)$.

2.20. Soit $\mathscr{A}$ une catégorie abélienne tensorielle, autrement dit $\mathscr{A}$ est une catégorie abélienne munie d'un foncteur biadditif $\otimes: \mathscr{A} \times \mathscr{A} \rightarrow \mathscr{A}$ et d'un objet unité $A$, vérifiant certaines conditions [10,1.15], et soit $\varphi: \mathfrak{o} \rightarrow \operatorname{End}(A)$ un homomorphisme. On a un homomorphisme canonique $\operatorname{End}(A) \rightarrow \operatorname{End}\left(\operatorname{id}_{\mathscr{A}}\right)$. 
On peut donc définir la catégorie $\alpha$ - $\mathscr{A}$ suivant 2.18. Le produit tensoriel induit un bifoncteur

$$
\alpha-\mathscr{A} \times \alpha-\mathscr{A} \rightarrow \alpha-\mathscr{A}, \quad(M, N) \mapsto M \otimes N,
$$

qui fait de $\alpha-\mathscr{A}$ une catégorie abélienne tensorielle, dont $A^{\alpha}$ est un objet unité (cf. $[3,1.4 .4])$. Le foncteur $\alpha$ induit un homomorphisme $\operatorname{End}(A) \rightarrow \operatorname{End}\left(A^{\alpha}\right)$. Par suite, pour tous objets $M$ et $N$ de $\alpha-\mathscr{A}, \operatorname{Hom}_{\alpha-\mathscr{A}}(M, N)$ est canoniquement muni d'une structure de $\operatorname{End}(A)$-module.

\section{Représentations à coefficients dans les modules de type $\alpha$-fini}

3.1. Rappelons que o désigne le séparé complété $p$-adique de $\mathcal{O}_{\bar{K}}$ et $\mathfrak{m}$ son idéal maximal et que l'on a $\mathfrak{m}^{2}=\mathfrak{m}$ et $v(\mathfrak{m})=\mathbb{Q}_{>0}$ (2.1). Pour tout nombre réel $r \geqslant 0$, on note $I_{r}$ l'idéal de $\mathfrak{o}$ formé des éléments $x \in \mathfrak{o}$ tels que $v(x)>r$.

On note $\operatorname{Mod}(\mathfrak{o})$ la catégorie des o-modules. Prenant pour $\varphi: \mathfrak{o} \stackrel{\sim}{\rightarrow}$ End( $\left.\mathrm{id}_{\operatorname{Mod}(\mathfrak{o})}\right)$ l'isomorphisme canonique, on considère les notions introduites dans (2.18) pour la catégorie $\operatorname{Mod}(\mathfrak{o})$. Considérons le foncteur

$$
\operatorname{Mod}(\mathfrak{o}) \rightarrow \operatorname{Mod}(\mathfrak{o}) \quad M \mapsto M_{\sharp}=\operatorname{Hom}_{\mathfrak{o}}(\mathfrak{m}, M) .
$$

Pour tout $\mathfrak{o}$-module $M$, on a des $\alpha$-isomorphismes (2.18) canoniques fonctoriels $[4, \mathrm{~V} .2 .4]$

$$
\begin{aligned}
M & \rightarrow M_{\sharp}, \quad x \mapsto(m \in \mathfrak{m} \mapsto m x), \\
\mathfrak{m} \otimes_{\mathfrak{o}} M & \rightarrow M .
\end{aligned}
$$

Proposition 3.2 [4, V.2.5 et 2.6]. Soit $f: M \rightarrow N$ un morphisme de o-module.

(i) Pour que $f$ soit un $\alpha$-isomorphisme, il faut et il suffit que le morphisme induit $f_{\sharp}: M_{\sharp} \rightarrow N_{\sharp}$ soit un isomorphisme.

(ii) Pour que $f$ soit un $\alpha$-isomorphisme, il faut et il suffit que le morphisme induit $\mathfrak{m} \otimes_{\mathfrak{o}} M \rightarrow \mathfrak{m} \otimes_{\mathfrak{o}} N$ soit un isomorphisme.

(iii) Pour tout entier $i \geqslant 0$, le foncteur $\operatorname{Ext}_{\mathfrak{o}}^{i}(\mathfrak{m},-)$ envoie les $\alpha$-isomorphismes sur des isomorphismes.

(iv) Le foncteur (3.1.1) envoie les $\alpha$-monomorphismes sur des monomorphismes.

REMARQUE 3.3. Le $\mathfrak{o}$-module $\mathfrak{m}$ n'est pas projectif. En effet, le $\mathfrak{o}$-module $\mathfrak{m}$ est plat et on a $\mathfrak{m}=\lim _{n \in \mathbb{N}}\left(p^{1 / n}\right)$, où $p^{1 / n}$ est une racine $n$-ième de $p$ dans $\mathcal{O}_{\bar{K}}$ (2.1). Mais le système projectif $\left(\operatorname{Hom}_{\mathfrak{o}}\left(\mathfrak{o},\left(p^{1 / n}\right)\right)\right)_{n \geqslant 1}$ ne vérifie pas la condition de Mittag-Leffler. L'assertion résulte alors de [39, 3.1.3]. 
3.4. Suivant [41, 2.2], pour tous $\mathfrak{o}$-modules $M$ et $N$ et tout $\gamma \in \mathfrak{m}$, on dit que $M$ et $N$ sont $\gamma$-équivalents et on note $M \approx_{\gamma} N$ s'il existe deux o-morphismes $f: M \rightarrow N$ et $g: N \rightarrow M$ tels que $f \circ g=\gamma \operatorname{id}_{N}$ et $g \circ f=\gamma \operatorname{id}_{M}$. On dit que $M$ et $N$ sont $\alpha$-équivalents et on note $M \approx N$ si $M$ et $N$ sont $\gamma$-équivalents pour tout $\gamma \in \mathfrak{m}$.

On dit qu'un $\mathfrak{o}$-module $M$ est de type $\alpha$-fini si, pour tout $\gamma \in \mathfrak{m}$, il existe un o-module de type fini $N$ tel que $M \approx_{\gamma} N$. Pour tout entier $n \geqslant 1$, on dit qu'un $\mathfrak{o}_{n}$-module $M$ est de type $\alpha$-fini s'il est de type $\alpha$-fini en tant que $\mathfrak{o}$-module.

On dit qu'une suite de $\mathfrak{o}$-modules est $\alpha$-exacte si ses groupes de cohomologie sont $\alpha$-nuls (cf. [3, 1.5.2]).

REMARQue 3.5. (i) D'après 2.19, deux o-modules $\alpha$-isomorphes sont $\alpha$ équivalents. La réciproque n'est pas vraie. En effet, pour tout nombre réel $r>0$, on a alors $I_{r} \approx \mathfrak{o}$ (3.1). Si $r \notin \mathbb{Q}_{>0}$, o et $I_{r}$ ne sont pas $\alpha$-isomorphes (cf. [3, 1.8.4]).

(ii) La notion de type $\alpha$-fini introduite dans 3.4 est compatible avec la définition générale introduite dans 12.28 (cf. [3, 1.5.3 et 1.8.5(ii)]).

ThÉORÈme $3.6[41,2.5]$. Pour tout o-module de type $\alpha$-fini $M$, il existe une unique suite décroissante de nombres réels positifs $\left(r_{i}\right)_{i \geqslant 1}$, tendant vers 0 , et un unique entier $n \geqslant 0$ tels que

$$
M \approx \mathfrak{o}^{n} \oplus\left(\oplus_{i \geqslant 1} \mathfrak{o} / I_{r_{i}}\right)
$$

Lemme 3.7 [3, 1.5.13 et 1.8.7]. Soit $0 \rightarrow M^{\prime} \rightarrow M \rightarrow M^{\prime \prime} \rightarrow 0$ une suite $\alpha$ exacte de o-modules. Pour que $M$ soit de type $\alpha$-fini, il faut et il suffit que $M^{\prime}$ et $M^{\prime \prime}$ le soient.

Proposition 3.8. Soit $M$ un o-module de type $\alpha$-fini.

(i) Il existe un sous-o-module libre de type fini $M^{\circ}$ de $M$ tel que $M / M^{\circ}$ soit annulé par une puissance de $p$.

(ii) Les sous-modules $\left(p^{n} M^{\circ}\right)_{n \geqslant 1}$ forment un système fondamental de voisinages de 0 pour la topologie p-adique de $M$.

(iii) La topologie p-adique de $M$ est séparée et complète.

(iv) Soit $N$ un sous-module de M. La topologie p-adique de $N$ est induite par la topologie p-adique de $M$.

Preuve. (i) D'après 3.6, le o-module $M$ est $\alpha$-équivalent à un o-module $N=\mathfrak{o}^{n} \oplus\left(\oplus_{i \geqslant 1} \mathfrak{o} / I_{r_{i}}\right)$ avec $\left(r_{i}\right)_{i \geqslant 1}$ tendant vers 0 . Notons $N^{\circ}$ le sous-ó-module 
$\mathfrak{o}^{n}$ de $N$. Choisissons un élément $\gamma$ de $\mathfrak{m}$. Il existe des morphismes de $\mathfrak{o}$-modules $f: N \rightarrow M$ et $g: M \rightarrow N$ tels que $g \circ f=\gamma$ id $_{N}$ et $f \circ g=\gamma \mathrm{id}_{M}$. La restriction de $f$ à $N^{\circ}$ est donc injective. On prend pour $M^{\circ}$ le sous-o-module libre de type fini $f\left(N^{\circ}\right)$ de $M$. On a alors $g\left(M^{\circ}\right)=\gamma N^{\circ} \subset N^{\circ}$. Les morphismes $f$ et $g$ induisent une $\gamma$-équivalence entre $M / M^{\circ}$ et $N / N^{\circ}$ :

$$
\bar{f}: N / N^{\circ} \rightarrow M / M^{\circ} \quad \bar{g}: M / M^{\circ} \rightarrow N / N^{\circ} .
$$

Comme $N / N^{\circ} \simeq \oplus_{i \geqslant 1} \mathfrak{o} / I_{r_{i}}$ est annulé par une puissance de $p$, il en est de même de $M / M^{\circ}$.

(ii) En vertu de (i), il existe un entier $m \geqslant 1$ tel que $p^{m}\left(M / M^{\circ}\right)=0$. On en déduit que $p^{m} M \subset M^{\circ} \subset M$. Les systèmes fondamentaux $\left(p^{n} M^{\circ}\right)_{n \geqslant 1}$ et $\left(p^{n} M\right)_{n \geqslant 1}$ de voisinages de 0 sont alors équivalents.

(iii) On a un isomorphisme canonique $M^{\circ} \stackrel{\sim}{\rightarrow} \widehat{M}^{\circ}=\lim M^{\circ} / p^{n} M^{\circ}$. En vertu de (ii), $\widehat{M}=\lim M / p^{n} M^{\circ}$ est le séparé complété $p$-adique de $M$. Pour tout entier $n \geqslant 1$, on a une suite exacte

$$
0 \rightarrow M^{\circ} / p^{n} M^{\circ} \rightarrow M / p^{n} M^{\circ} \rightarrow M / M^{\circ} \rightarrow 0 .
$$

Le système projectif $\left(M^{\circ} / p^{n} M^{\circ}\right)_{n \geqslant 1}$ vérifie la condition de Mittag-Leffler. La limite projective induit donc une suite exacte

$$
0 \rightarrow \widehat{M^{\circ}} \rightarrow \widehat{M} \rightarrow M / M^{\circ} \rightarrow 0
$$

qui s'insère dans un diagramme commutatif

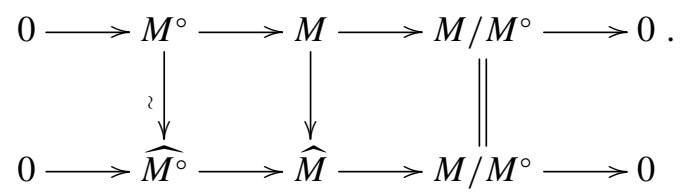

On en déduit que le morphisme canonique $M \rightarrow \widehat{M}$ est un isomorphisme; d'où l'assertion.

(iv) En vertu de 3.7, $N$ est de type $\alpha$-fini. Il existe un sous-o-module libre de type fini $N^{\circ}$ de $N$ vérifiant la condition (i). On peut supposer de plus que $N^{\circ} \subset M^{\circ}$. Soit $N_{\text {sat }}^{\circ}=\left\{x \in M^{\circ} \mid p^{n} x \in N^{\circ}\right.$ pour un entier $\left.n \geqslant 1\right\}$ la saturation de $N^{\circ}$ dans $M^{\circ}$. On a alors $\left(p^{n} M^{\circ}\right) \cap N_{\text {sat }}^{\circ}=p^{n} N_{\text {sat }}^{\circ}$ pour tout $n \geqslant 1$. Comme $p$ n'est pas un diviseur de zéro dans $M^{\circ} / N_{\text {sat }}^{\circ}, M^{\circ} / N_{\text {sat }}^{\circ}$ est o-plat [2, 1.9.12]. On en déduit que $N_{\text {sat }}^{\circ}$ est de type fini $[2,1.9 .14]$. Il existe donc un entier $l \geqslant 1$ tel que $p^{l} N_{\text {sat }}^{\circ} \subset N^{\circ}$. Pour tout entier $n \geqslant 1$, on a

$$
\left(p^{n} M^{\circ}\right) \cap N^{\circ}=\left(p^{n} M^{\circ} \cap N_{\text {sat }}^{\circ}\right) \cap N^{\circ}=\left(p^{n} N_{\text {sat }}^{\circ}\right) \cap N^{\circ}=p^{n} N_{\text {sat }}^{\circ} .
$$


On en déduit que

$$
p^{n} N^{\circ} \subset\left(p^{n} M^{\circ}\right) \cap N^{\circ} \subset p^{n-l} N^{\circ} .
$$

L'assertion s'ensuit compte tenu de (ii).

Lemme 3.9 [3, 1.8.11]. Soit $M$ un o-module tel que $M / p M$ soit de type $\alpha$-fini. Alors, le o-module $\widehat{M}=\lim _{\longleftarrow} M / p^{n} M$ est de type $\alpha-f i n i$.

Lemme 3.10. (i) Soit $\left(M_{n}\right)_{n \geqslant 1}$ un système projectif de o-modules $\alpha$-nuls. Alors, les o-modules $\lim _{\leftarrow} M_{n}$ et $\mathrm{R}^{1} \underset{\lim }{\longleftarrow} M_{n}$ sont $\alpha$-nuls.

(ii) Soit $\left(M_{n}\right)_{n \geqslant 1}$ et $\left(N_{n}\right)_{n \geqslant 1}$ deux systèmes projectifs de o-modules et $\left(f_{n}: M_{n} \rightarrow\right.$ $\left.N_{n}\right)_{n \geqslant 1}$ un système projectif des $\alpha$-isomorphismes. Alors, le foncteur limite projective induit des $\alpha$-isomorphismes $\lim _{\longleftarrow} M_{n} \rightarrow \underset{\lim }{\lim } N_{n}$ et $\mathrm{R}^{1} \lim _{\leftarrow} M_{n} \rightarrow$ $\mathrm{R}^{1} \lim _{\leftarrow} N_{n}$.

L'assertion (i) est démontrée dans [18, 2.4.2] et l'assertion (ii) résulte de (i).

DÉfinition 3.11 [4, V.6.1]. Soit $R$ une $\mathfrak{o}$-algèbre et $M$ un $R$-module. On dit que $M$ est $\alpha$-plat s'il vérifie les conditions équivalentes suivantes.

(i) Pour tout $R$-module $N$ et tout entier $i \geqslant 1$, $\operatorname{Tor}_{i}^{R}(M, N)$ est un o-module $\alpha$-nul.

(ii) Pour tout $R$-module $N$, $\operatorname{Tor}_{1}^{R}(M, N)$ est un $\mathfrak{o}$-module $\alpha$-nul.

(iii) Pour tout morphisme $R$-linéaire injectif $f: N_{1} \rightarrow N_{2}$, le noyau de $\mathrm{id}_{M} \otimes f: M \otimes_{R} N_{1} \rightarrow M \otimes_{R} N_{2}$ est un o-module $\alpha$-nul.

LEMme 3.12. Soit $R$ une o-algèbre et $M$ un $R$-module $\alpha$-plat. Alors, le $R$-module $\mathfrak{m} \otimes_{\mathfrak{o}} M$ est plat. En particulier, pour tout $\mathfrak{o}$-module $\alpha$-plat $N, \mathfrak{m} \otimes_{\mathfrak{o}} N$ est sans torsion.

Preuve. En vertu de 3.2(ii), pour tout $\mathfrak{o}$-module $\alpha$-nul $N, \mathfrak{m} \otimes_{\mathfrak{o}} N$ est nul. La $R$ platitude de $\mathfrak{m} \otimes_{\mathfrak{o}} M$ résulte alors de la $\mathfrak{o}$-platitude de $\mathfrak{m}$. On sait qu'un $\mathfrak{o}$-module est plat si et seulement s'il est sans torsion [8, chap. VI, Section 3.6, lem. 1]; d'où la deuxième assertion.

Proposition 3.13. Soit $M$ et $N$ deux $\mathfrak{o}_{n}$-modules (2.1) (resp. o-modules) $\alpha$ équivalents. Alors, la $\alpha$-platitude de $M$ est équivalente à celle de $N$.

Preuve. Supposons que $M$ soit $\alpha$-plat. Soit $f: N_{1} \rightarrow N_{2}$ un morphisme injectif de $\mathfrak{o}_{n}$-modules. Pour tout $\gamma \in \mathfrak{m}$, il existe des morphismes o-linéaires $g: M \rightarrow N$ et $h: N \rightarrow M$ tels que $g \circ h=\gamma \mathrm{id}_{N}$ et $h \circ g=\gamma \mathrm{id}_{M}$. On a alors un 
diagramme commutatif

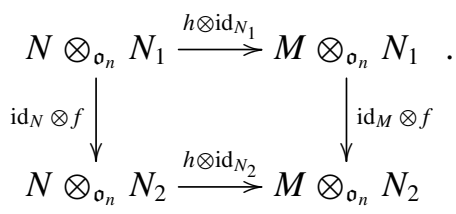

Comme $\operatorname{Ker}\left(M \otimes_{\mathfrak{o}_{n}} N_{1} \rightarrow M \otimes_{\mathfrak{o}_{n}} N_{2}\right)$ est $\alpha$-nul et $g \circ h=\gamma \mathrm{id}_{N}$, on en déduit que $\operatorname{Ker}\left(N \otimes_{\mathfrak{o}_{n}} N_{1} \rightarrow N \otimes_{\mathfrak{o}_{n}} N_{2}\right)$ est annulé par l'idéal $\gamma \mathfrak{m}$ de $\mathfrak{o}$. La $\alpha$-platitude de $N$ s'ensuit compte tenu du fait que $\mathfrak{m}^{2}=\mathfrak{m}$. La démonstration pour les $\mathfrak{o}$-modules est similaire.

Corollaire 3.14. Soit $n$ un entier $\geqslant 1$ et $M$ un $\mathfrak{o}_{n}$-module (resp. o-module) de type $\alpha$-fini. Pour que $M$ soit $\alpha$-plat, il faut et il suffit qu'il existe un entier $m \geqslant 0$ tel que $M \approx \mathfrak{o}_{n}^{m}$ (resp. $\left.M \approx \mathfrak{o}^{m}\right)$.

Preuve. Comme $p^{n} M=0$, d'après 3.6, il existe une suite décroissante de nombres réels $\left(r_{i}\right)_{i \geqslant 1}$ tendant vers 0 tels que $r_{i} \leqslant n$ et que $M \approx \oplus_{i \geqslant 1} \mathfrak{o} / I_{r_{i}}$. Le $\mathfrak{o}$-module $\mathfrak{o} / I_{n}$ est $\alpha$-isomorphe à $\mathfrak{o}_{n}$. Soit $r$ un nombre réel tel que $0<r<n$. Il est clair que $\mathfrak{o} / I_{r}$ n'est pas un $\mathfrak{o}_{n}$-module $\alpha$-plat. L'assertion pour les $\mathfrak{o}_{n}$-modules s'ensuit. La démonstration pour les $\mathfrak{o}$-modules est similaire.

LEMME 3.15. Soit $\left(M_{n}\right)_{n \geqslant 1}$ un système projectif de o-modules tel que, pour tout entier $n \geqslant 1, M_{n}$ soit un $\mathfrak{o}_{n}$-module $\alpha$-plat de type $\alpha$-fini et que le morphisme canonique $M_{n+1} \otimes_{\mathfrak{o}_{n+1}} \mathfrak{o}_{n} \rightarrow M_{n}$ soit un $\alpha$-isomorphisme. On pose, pour tout entier $n \geqslant 1, N_{n}=\mathfrak{m} \otimes_{\mathfrak{o}} M_{n}, \widehat{M}=\lim _{\longleftarrow} M_{n}$ et $\widehat{N}=\lim N_{n}$. Alors :

(i) on a un $\alpha$-isomorphisme canonique $\widehat{N} \rightarrow \widehat{M}$;

(ii) l'homomorphisme canonique $\widehat{N} / p^{n} \widehat{N} \rightarrow N_{n}$ est un isomorphisme;

(iii) les o-modules $\widehat{N}$ et $\widehat{M}$ sont $\alpha$-plats de type $\alpha$-fini.

Preuve. (i) Pour tout entier $n \geqslant 1$, le morphisme canonique $N_{n} \rightarrow M_{n}$ est un $\alpha$-isomorphisme (3.1.3). En vertu de 3.10(ii), la limite projective induit un $\alpha$ isomorphisme canonique $\widehat{N} \rightarrow \widehat{M}$.

(ii) D'après 3.2(ii), le $\alpha$-isomorphisme $M_{n+1} \otimes_{\mathfrak{o}_{n+1}} \mathfrak{o}_{n} \rightarrow M_{n}$ induit un isomorphisme $N_{n+1} \otimes_{\mathfrak{o}_{n+1}} \mathfrak{o}_{n} \stackrel{\sim}{\rightarrow} N_{n}$. On notera que, pour tout entier $n \geqslant 1, N_{n}$ est un $\mathfrak{o}_{n}$-module plat (3.12). Pour tous entiers $m, n \geqslant 1$, appliquant le foncteur 
$N_{m+n} \otimes_{\mathfrak{o}_{m+n}}-$ à la suite exacte $0 \rightarrow \mathfrak{o}_{m} \stackrel{p^{n}}{\rightarrow} \mathfrak{o}_{m+n} \rightarrow \mathfrak{o}_{n} \rightarrow 0$, on en déduit une suite exacte :

$$
0 \rightarrow N_{m} \stackrel{p^{n}}{\rightarrow} N_{m+n} \rightarrow N_{n} \rightarrow 0 .
$$

Le système projectif $\left(N_{m}\right)_{m \geqslant 1}$ vérifie la condition de Mittag-Leffler. On en déduit une suite exacte

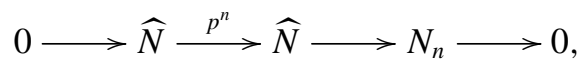

d'où l'assertion.

(iii) En vertu de (i) et 3.14, il suffit de démontrer l'assertion pour $\widehat{N}$. La $\alpha$ finitude de $\widehat{N}$ résulte de (ii) et 3.9. D'après 3.6, $\widehat{N}$ est $\alpha$-équivalent à un o-module $\mathfrak{o}^{m} \oplus\left(\oplus_{i \geqslant 1} \mathfrak{o} / I_{r_{i}}\right)$ avec $\left(r_{i}\right)_{i \geqslant 1}$ tendant vers 0 . On en déduit que, pour tout entier $n \geqslant 1$, on a $\widehat{N} / p^{n} \widehat{N} \approx \mathfrak{o}_{n}^{m} \oplus\left(\oplus_{i \geqslant 1}\left(\mathfrak{o} / I_{r_{i}}\right) / p^{n}\left(\mathfrak{o} / I_{r_{i}}\right)\right)$. D'autre part, on notera qu'il existe un entier $l \geqslant 1$ tel que, pour tout entier $n \geqslant 1, N_{n} \approx \mathfrak{o}_{n}^{l}$ (3.14). En vertu de (ii), pour tout entier $i \geqslant 1$, on a donc $r_{i}=0$; d'où la $\alpha$-platitude de $\widehat{N}$.

REMARQUE 3.16. Sous les hypothèses de 3.15, la topologie $p$-adique de $\widehat{N}$ est la limite projective des topologies discrètes sur les $N_{n}$.

3.17. Soit $V$ un $\mathfrak{C}$-espace vectoriel de dimension finie, $V^{\circ}$ un sous-o-module libre de type fini de $V$ qui l'engendre sur $\mathfrak{C}$. On appelle topologie p-adique sur $V$ l'unique topologie compatible avec sa structure de groupe additif pour laquelle les sous-groupes $\left(p^{n} V^{\circ}\right)_{n \geqslant 1}$ forment un système fondamental de voisinages de 0 . Cette topologie ne dépend pas du choix de $V^{\circ}$ (cf. [4, II.2.2]). On notera que tout morphisme $\mathfrak{C}$-linéaire de $\mathfrak{C}$-espaces vectoriels de dimension finie est continu pour la topologie $p$-adique.

3.18. Dans la suite de cette section, on se donne un groupe profini $G$. Pour tout entier $n \geqslant 1$, on munit l'anneau $\mathfrak{o}_{n}=\mathfrak{o} / p^{n} \mathfrak{o}$ (resp. $\mathfrak{o}$, resp. $\mathfrak{C}$ ) de la topologie discrète (resp. de la topologie $p$-adique, resp. de la topologie $p$-adique (3.17)) et de l'action triviale de $G$.

Soit $n$ un entier $\geqslant 1$. Par $\mathfrak{o}_{n}$-représentation de $G$, on sous-entend un $\mathfrak{o}_{n}$-module $M$ muni de la topologie discrète et d'une action continue et $\mathfrak{o}_{n}$-linéaire de $G$. Un morphisme de $\mathfrak{o}_{n}$-représentations de $G$ est une application $\mathfrak{o}_{n}$-linéaire et $G$ équivariante. On désigne par $\operatorname{Rep}_{\mathfrak{o}_{n}}(G)$ la catégorie des $\mathfrak{o}_{n}$-représentations de $G$.

On note $\breve{\mathfrak{o}}$ le système projectif d'anneaux $\left(\mathfrak{o}_{n}\right)_{n \geqslant 1}$. On désigne par $\operatorname{Rep}_{\breve{\mathfrak{o}}}(G)$ la catégorie des systèmes projectifs de $\mathfrak{o}[G]$-modules discrets $\left(V_{n}\right)_{n \geqslant 1}$ tels que, pour tout entier $n \geqslant 1, p^{n} V_{n}=0$. Il est clair que les catégories $\operatorname{Rep}_{\mathfrak{o}_{n}}(G)$ et $\operatorname{Rep}_{\mathfrak{o}}(G)$ sont abéliennes. 
Par o-représentation continue de $G$, on sous-entend un o-module de type $\alpha$-fini muni de la topologie $p$-adique et d'une action o-linéaire continue de $G$. Par $\mathfrak{C}$ représentation continue, on sous-entend un $\mathfrak{C}$-espace vectoriel de dimension finie muni de la topologie $p$-adique (3.17) et d'une action $\mathfrak{C}$-linéaire continue de $G$. Un morphisme de $\mathfrak{o}$-représentations (resp. $\mathfrak{C}$-représentations) continues de $G$ est une application o-linéaire et $G$-équivariante. Une telle application est continue pour la topologie $p$-adique (cf. 3.17). On désigne par $\operatorname{Rep}_{\mathfrak{o}}^{\text {cont }}(G)\left(\operatorname{resp} . \operatorname{Rep}_{\mathfrak{C}}^{\text {cont }}(G)\right)$ la catégorie des $\mathfrak{o}$-représentations (resp. $\mathfrak{C}$-représentations) continues de $G$.

Lemme 3.19. Soit $V$ un objet de $\operatorname{Rep}_{\mathfrak{o}}^{\text {cont }}(G)$ et $W$ un sous-o-module de $V$ stable par G. Alors, $W$ (resp. $V / W$ ) est un o-module de type $\alpha$-fini et la restriction de l'action de $G$ sur $W$ (resp. $V / W$ ) est continue pour la topologie p-adique.

Preuve. La $\alpha$-finitude de $W$ et $V / W$ résulte de 3.7. La continuité de l'action de $G$ pour la topologie $p$-adique de $W$ s'ensuit compte tenu de 3.8(iv). Soit $\pi: V \rightarrow$ $V / W$ la projection. Pour tout entier $n \geqslant 1$, on a $\pi\left(p^{n} V\right)=p^{n}(V / W)$. On en déduit la continuité de l'action de $G$ sur $V / W$.

Proposition 3.20. La catégorie $\operatorname{Rep}_{\mathfrak{o}}^{\text {cont }}(G)$ est abélienne.

Preuve. Cette catégorie est clairement additive. Soit $f: V_{1} \rightarrow V_{2}$ un morphisme de $\operatorname{Rep}_{\mathfrak{o}}^{\text {cont }}(G)$. D'après 3.19, on définit $\operatorname{Ker}(f)$ (resp. Coker $\left.(f)\right)$ par le o-module $\operatorname{Ker}\left(V_{1} \rightarrow V_{2}\right)$ (resp. $\left.\operatorname{Coker}\left(V_{1} \rightarrow V_{2}\right)\right)$ muni de la topologie $p$-adique et de l'action de $G$ induite par $V_{1}$ (resp. $V_{2}$ ). Il est clair que le morphisme canonique $\operatorname{Coim}(f) \rightarrow \operatorname{Im}(f)$ est un isomorphisme.

3.21. Soit $n$ un entier $\geqslant 1$. On désigne par $\operatorname{Rep}_{\mathfrak{o}_{n}}^{\text {lff }}(G)$ la sous-catégorie pleine de $\operatorname{Rep}_{\mathfrak{o}_{n}}(G)$ formée des $\mathfrak{o}_{n}$-représentations dont le $\mathfrak{o}_{n}$-module sous-jacent est libre de type fini et par $\operatorname{Rep}_{\mathfrak{o}}^{\text {ltf }}(G)$ la sous-catégorie pleine de $\operatorname{Rep}_{\mathfrak{o}}^{\text {cont }}(G)$ formée des $\mathfrak{o}$-représentations continues dont le $\mathfrak{o}$-module sous-jacent est libre de type fini.

On désigne par $\boldsymbol{R e p}_{\breve{o}}^{\text {lff }}(G)$ la sous-catégorie pleine de $\boldsymbol{R e p}_{\breve{\mathfrak{o}}}(G)$ formée des systèmes projectifs $\left(V_{n}\right)_{n \geqslant 1}$ tels que, pour tout entier $n \geqslant 1, V_{n}$ soit un objet de $\boldsymbol{R e p}_{\mathfrak{o}_{n}}^{\text {lff }}(G)$ et que le morphisme canonique $V_{n+1} \otimes_{\mathfrak{o}_{n+1}} \mathfrak{o}_{n} \stackrel{\sim}{\rightarrow} V_{n}$ soit un isomorphisme de $\mathfrak{o}_{n}$-représentations de $G$.

Le foncteur limite projective induit une équivalence de catégories

$$
\operatorname{Rep}_{\breve{o}}^{\text {ltf }}(G) \stackrel{\sim}{\rightarrow} \operatorname{Rep}_{\mathfrak{o}}^{\text {ltf }}(G)
$$

En effet, le foncteur $\operatorname{Rep}_{\mathfrak{o}}^{\text {ltf }}(G) \rightarrow \operatorname{Rep}_{\breve{o}}^{\text {ltf }}(G)$, défini par $V \mapsto\left(V / p^{n} V\right)_{n \geqslant 1}$, est un quasi-inverse de (3.21.1). Pour tout objet $V$ de $\operatorname{Rep}_{\mathfrak{o}}^{\text {ltf }}(G)$, on note $V\left[\frac{1}{p}\right]$ la $\mathfrak{C}$ représentation continue associée. Le foncteur canonique $\operatorname{Rep}_{\mathfrak{o}}^{\text {lff }}(G) \rightarrow \operatorname{Rep}_{\mathfrak{C}}^{\text {cont }}(G)$ 
induit une équivalence de catégories :

$$
\operatorname{Rep}_{\mathfrak{o}}^{\text {ltf }}(G)_{\mathbb{Q}} \stackrel{\sim}{\rightarrow} \operatorname{Rep}_{\mathfrak{C}}^{\text {cont }}(G),
$$

où la source désigne la catégorie des objets de $\operatorname{Rep}_{\mathfrak{o}}^{\text {ltf }}(G)$ à isogénie près (cf. [12, prop. 22]).

3.22. Prenant pour $\varphi$ l'un des homomorphismes canoniques $\mathfrak{o} \rightarrow$ End

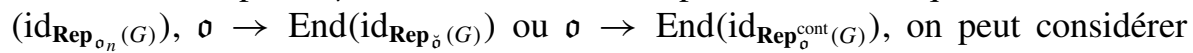
les notions introduites dans 2.18 pour les catégories abéliennes $\operatorname{Rep}_{\mathfrak{o}_{n}}(G)$, $\operatorname{Rep}_{\breve{o}}(G)$ et $\boldsymbol{R e p}_{\mathfrak{o}}^{\text {cont }}(G)$. Un morphisme de $\boldsymbol{R e p}_{\mathfrak{o}_{n}}(G)\left(\operatorname{resp} . \operatorname{Rep}_{\mathfrak{o}}^{\text {cont }}(G)\right)$ est un $\alpha$-isomorphisme si et seulement s'il induit un $\alpha$-isomorphisme sur les modules sous-jacents. Un morphisme $\left(f_{n}\right)_{n \geqslant 1}$ de $\operatorname{Rep}_{\breve{\mathfrak{o}}}(G)$ est un $\alpha$-isomorphisme si et seulement si, pour tout entier $n \geqslant 1, f_{n}$ est un $\alpha$-isomorphisme de $\operatorname{Rep}_{\mathfrak{o}_{n}}(G)$.

3.23. Soit $n$ un entier $\geqslant 1$. On désigne par $\operatorname{Rep}_{\mathfrak{o}_{n}}^{\alpha \text { ptf }}(G)$ la sous-catégorie pleine de $\operatorname{Rep}_{\mathfrak{o}_{n}}(G)$ formée des $\mathfrak{o}_{n}$-représentations dont le $\mathfrak{o}_{n}$-module sous-jacent est $\alpha$ plat de type $\alpha$-fini (3.14) et par $\operatorname{Rep}_{\mathfrak{o}}^{\alpha \text { ptf }}(G)$ la sous-catégorie pleine de $\operatorname{Rep}_{\mathfrak{o}}^{\text {cont }}(G)$ formée des o-représentations continues de $G$ dont le o-module sous-jacent est $\alpha$-plat de type $\alpha$-fini.

On désigne par $\operatorname{Rep}_{\breve{\mathfrak{o}}}^{\alpha \text { ptf }}(G)$ la sous-catégorie pleine de $\operatorname{Rep}_{\breve{\mathfrak{o}}}(G)$ formée des systèmes projectifs de représentations $\left(V_{n}\right)_{n \geqslant 1}$ tels que, pour tout entier $n \geqslant 1, V_{n}$ soit un objet de $\operatorname{Rep}_{\mathfrak{o}_{n}}^{\alpha \text { ptf }}(G)$ et que le morphisme canonique $V_{n+1} \otimes_{\mathfrak{o}_{n+1}} \mathfrak{o}_{n} \rightarrow V_{n}$ soit un $\alpha$-isomorphisme (3.22).

En munissant $\mathfrak{m}$ de l'action triviale de $G$, d'après 3.15 et 3.16 , le foncteur limite projective induit un foncteur

$$
F_{1}: \operatorname{Rep}_{\mathfrak{o}}^{\alpha \mathrm{ptf}}(G) \rightarrow \operatorname{Rep}_{\mathfrak{o}}^{\alpha \mathrm{ptf}}(G) \quad\left(V_{n}\right)_{n \geqslant 1} \mapsto \lim _{\longleftarrow} \mathfrak{m} \otimes_{\mathfrak{o}} V_{n} .
$$

D'autre part, on a un foncteur

$$
F_{2}: \operatorname{Rep}_{\mathfrak{o}}^{\alpha \mathrm{ptf}}(G) \rightarrow \operatorname{Rep}_{\mathfrak{o}}^{\alpha \mathrm{ptf}}(G) \quad V \mapsto\left(V / p^{n} V\right)_{n \geqslant 1} .
$$

En vertu de 3.8(i)-(ii), on a un foncteur canonique

$$
\operatorname{Rep}_{\mathfrak{o}}^{\alpha \text { ptf }}(G) \rightarrow \operatorname{Rep}_{\mathfrak{C}}^{\text {cont }}(G) \quad V \mapsto V\left[\frac{1}{p}\right]
$$

On désigne par $\operatorname{Rep}_{\mathfrak{o}}^{\alpha \text { ptf }}(G)_{\mathbb{Q}}$ (resp. $\operatorname{Rep}_{\mathfrak{o}}^{\alpha \text { ptf }}(G)_{\mathbb{Q}}$ ) la catégorie des objets de $\operatorname{Rep}_{\mathfrak{o}}^{\alpha p t f}(G)\left(\operatorname{resp} . \operatorname{Rep}_{\breve{o}}^{\alpha p t f}(G)\right)$ à isogénie près.

LEMME 3.24. (i) Le foncteur $F_{1}$ envoie les $\alpha$-isomorphismes sur des isomorphismes. 
(ii) Soit $\left(V_{n}\right)_{n \geqslant 1}$ un objet de $\operatorname{Rep}_{\breve{o}}^{\alpha \mathrm{ptf}}(G)$. On a un isomorphisme canonique fonctoriel

$$
F_{2}\left(F_{1}\left(\left(V_{n}\right)_{n \geqslant 1}\right)\right) \stackrel{\sim}{\rightarrow}\left(\mathfrak{m} \otimes_{\mathfrak{o}} V_{n}\right)_{n \geqslant 1}
$$

et un $\alpha$-isomorphisme canonique $\left(\mathfrak{m} \otimes_{\mathfrak{o}} V_{n}\right)_{n \geqslant 1} \rightarrow\left(V_{n}\right)_{n \geqslant 1}$.

Preuve. L'assertion (i) résulte de 3.2(ii) et l'assertion (ii) résulte de (3.1.3) et 3.15(ii).

LEMME 3.25. Soit $V$ un objet de $\operatorname{Rep}_{\mathfrak{o}}^{\alpha \mathrm{ptf}}(G)$. On munit $\mathfrak{m} \otimes_{\mathfrak{o}} V$ de la topologie p-adique et de l'action canonique de G. Alors :

(i) on a un isomorphisme canonique fonctoriel $\mathfrak{m} \otimes_{\mathfrak{o}} V \stackrel{\sim}{\rightarrow} F_{1}\left(F_{2}(V)\right)$;

(ii) le $\alpha$-isomorphisme canonique $\mathfrak{m} \otimes_{\mathfrak{o}} V \rightarrow V$ est injectif.

Preuve. (i) D'après 3.8(iii), on a un isomorphisme canonique et fonctoriel de $\mathfrak{o}$-représentations continues $\mathfrak{m} \otimes_{\mathfrak{o}} V \stackrel{\sim}{\rightarrow} \widehat{\mathfrak{m} \otimes_{\mathfrak{o}} V} \simeq \lim \mathfrak{m} \otimes_{\mathfrak{o}}\left(V / p^{n} V\right)$; d'où l'assertion.

(ii) Le noyau du morphisme $\mathfrak{m} \otimes_{\mathfrak{o}} V \rightarrow V$ est $\alpha$-nul. La proposition résulte du fait que le $\mathfrak{o}$-module $\mathfrak{m} \otimes_{\mathfrak{o}} V$ n'a pas de torsion (3.12).

Corollaire 3.26. Les foncteurs $F_{1}$ et $F_{2}$ induisent des équivalences de catégories quasi inverses l'une de l'autre

$$
\operatorname{Rep}_{\mathfrak{o}}^{\alpha \mathrm{ptf}}(G)_{\mathbb{Q}} \stackrel{\sim}{\rightarrow} \operatorname{Rep}_{\mathfrak{\mathfrak { o }}}^{\alpha \mathrm{ptf}}(G)_{\mathbb{Q}}, \quad \operatorname{Rep}_{\mathfrak{\mathfrak { o }}}^{\alpha \mathrm{ptf}}(G)_{\mathbb{Q}} \stackrel{\sim}{\rightarrow} \operatorname{Rep}_{\mathfrak{o}}^{\alpha \mathrm{ptf}}(G)_{\mathbb{Q}} .
$$

Preuve. En vertu de 2.19, tout $\alpha$-isomorphisme est une isogénie. L'assertion résulte alors de 3.24 et 3.25 .

Proposition 3.27. Le foncteur canonique (3.23.3) induit une équivalence de catégories

$$
\operatorname{Rep}_{\mathfrak{o}}^{\alpha \mathrm{ptf}}(G)_{\mathbb{Q}} \rightarrow \operatorname{Rep}_{\mathfrak{C}}^{\mathrm{cont}}(G) .
$$

Preuve. L'essentielle surjectivité résulte de (3.21.2). Soit $V_{1}$ et $V_{2}$ deux objets de $\operatorname{Rep}_{\mathfrak{o}}^{\alpha \text { ptf }}(G)$. Il suffit de démontrer que le morphisme canonique

$$
\operatorname{Hom}_{G}\left(V_{1}, V_{2}\right) \otimes_{\mathbb{Z}} \mathbb{Q} \rightarrow \operatorname{Hom}_{G}\left(V_{1}\left[\frac{1}{p}\right], V_{2}\left[\frac{1}{p}\right]\right)
$$

est un isomorphisme. D'après 3.25(ii), on a une suite exacte de $\operatorname{Rep}_{\mathfrak{o}}^{\text {cont }}(G)$

$$
0 \rightarrow \mathfrak{m} \otimes_{\mathfrak{o}} V_{2} \rightarrow V_{2} \rightarrow k \otimes_{\mathfrak{o}} V_{2} \rightarrow 0
$$


On en déduit une suite exacte

$$
0 \rightarrow \operatorname{Hom}_{G}\left(V_{1}, \mathfrak{m} \otimes_{\mathfrak{o}} V_{2}\right) \rightarrow \operatorname{Hom}_{G}\left(V_{1}, V_{2}\right) \rightarrow \operatorname{Hom}_{G}\left(V_{1}, k \otimes_{\mathfrak{o}} V_{2}\right)
$$

Comme $k \otimes_{\mathfrak{o}} V_{2}$ est $\alpha$-nul, on a des isomorphismes $\left(\mathfrak{m} \otimes_{\mathfrak{o}} V_{2}\right)\left[\frac{1}{p}\right] \stackrel{\sim}{\rightarrow} V_{2}\left[\frac{1}{p}\right]$ et $\operatorname{Hom}_{G}\left(V_{1}, \mathfrak{m} \otimes_{\mathfrak{o}} V_{2}\right) \otimes_{\mathbb{Z}} \mathbb{Q} \stackrel{\sim}{\rightarrow} \operatorname{Hom}_{G}\left(V_{1}, V_{2}\right) \otimes_{\mathbb{Z}} \mathbb{Q}$. D'après 3.12 , on peut donc se ramener au cas où $V_{2}$ est sans torsion.

On note $V_{1 \text {,tor }}$ le sous-module de torsion de $V_{1}$ et on le munit de la topologie $p$-adique et de l'action continue de $G$ induite par $V_{1}$ (3.20). On a alors des isomorphismes

$$
\operatorname{Hom}_{G}\left(V_{1} / V_{1, \text { tor }}, V_{2}\right) \stackrel{\sim}{\rightarrow} \operatorname{Hom}_{G}\left(V_{1}, V_{2}\right)
$$

et $V_{1}\left[\frac{1}{p}\right] \stackrel{\sim}{\rightarrow}\left(V_{1} / V_{1, \text { tor }}\right)\left[\frac{1}{p}\right]$. On peut donc se ramener au cas où $V_{1}$ est sans torsion.

Comme $V_{1}$ et $V_{2}$ sont isogènes à des o-modules libres de type fini (3.14), on a un isomorphisme canonique

$$
\operatorname{Hom}_{\mathfrak{o}}\left(V_{1}, V_{2}\right) \otimes_{\mathbb{Z}} \mathbb{Q} \stackrel{\sim}{\rightarrow} \operatorname{Hom}_{\mathfrak{C}}\left(V_{1}\left[\frac{1}{p}\right], V_{2}\left[\frac{1}{p}\right]\right) .
$$

On en déduit l'injectivité de (3.27.2). Étant donné une application $\mathfrak{C}$-linéaire et $G$-équivariante $f: V_{1}\left[\frac{1}{p}\right] \rightarrow V_{2}\left[\frac{1}{p}\right]$, il existe une application o-linéaire $g: V_{1} \rightarrow V_{2}$ telle que $g\left[\frac{1}{p}\right]=f$. Comme les actions de $G$ sur $\mathfrak{o}$ et $\mathfrak{C}$ sont triviales et les o-modules sous-jacents à $V_{1}$ et $V_{2}$ n'ont pas de torsion, l'application $g$ est $G$-équivariante; d'où la surjectivité de (3.27.2).

Lemme 3.28. Soit $X$ un schéma connexe et $\bar{x}$ un point géométrique de $X$. Pour tout entier $n \geqslant 1$, on note encore $\mathfrak{o}_{n}$ le faisceau d'anneaux constant de $X_{\text {fét }} d e$ valeur $\mathfrak{o}_{n}$ et on note $\breve{\mathfrak{o}}$ l'anneau $\left(\mathfrak{o}_{n}\right)_{n \geqslant 1}$ de $X_{\text {fét }}^{\mathbb{N}}$.

(i) Pour qu'un $\mathfrak{o}_{n}$-module $\mathbb{L}$ de $X_{\text {fét }}$ soit localement libre de type fini, il faut et il suffit que sa fibre $\mathbb{L}_{\bar{x}}$ en $\bar{x}$ soit un $\mathfrak{o}_{n}$-module libre de type fini.

(ii) Pour qu'un $\breve{\mathfrak{o}}$-module $\mathbb{L}=\left(\mathbb{L}_{n}\right)_{n \geqslant 1}$ soit localement libre de type fini, il faut et il suffit que $\mathbb{L}$ soit adique et que pour tout entier $n \geqslant 1$, le $\mathfrak{o}_{n}$-module $\mathbb{L}_{n}$ soit localement libre de type fini.

Preuve. L'assertion (i) est démontrée dans [4, III.2.11]. En calquant la démonstration de [4, III.7.19, 7.20], on vérifie l'assertion (ii).

3.29. Conservons les notations de 3.28 et reprenons de plus celles de 3.21 pour $G=\pi_{1}(X, \bar{x})$. Soit $n$ un entier $\geqslant 1$. La restriction du foncteur $\mu_{\bar{x}}: \mathbf{B}_{\pi_{1}(X, \bar{x})} \stackrel{\sim}{\rightarrow} X_{\text {fét }}$ 
(2.14.2) à la sous-catégorie $\operatorname{Rep}_{\mathfrak{o}_{n}}\left(\pi_{1}(X, \bar{x})\right)$ de $\mathbf{B}_{\pi_{1}(X, \bar{x})}$ induit une équivalence de catégories

$$
\operatorname{Rep}_{\mathfrak{o}_{n}}\left(\pi_{1}(X, \bar{x})\right) \stackrel{\sim}{\rightarrow} \operatorname{Mod}\left(X_{\text {fét }}, \mathfrak{o}_{n}\right) .
$$

D'après 3.28(i), celle-ci induit une équivalence de catégories $(2.9,3.21)$

$$
\mu_{\bar{x}}: \operatorname{Rep}_{\mathfrak{o}_{n}}^{\mathrm{ltf}}\left(\pi_{1}(X, \bar{x})\right) \stackrel{\sim}{\rightarrow} \mathbf{L} \mathbf{L}^{\mathrm{tf}}\left(X_{\text {fét }}, \mathfrak{o}_{n}\right) .
$$

D'après (3.21.1) et 3.28(ii), les foncteurs (3.29.2) induisent une équivalence de catégories

$$
\breve{\mu}_{\bar{x}}: \operatorname{Rep}_{\mathfrak{o}}^{\text {lff }}\left(\pi_{1}(X, \bar{x})\right) \stackrel{\sim}{\rightarrow} \mathbf{L L}^{\mathrm{tf}}\left(X_{\text {fét }}^{\mathbb{N}^{\circ}}, \breve{\mathfrak{o}}\right) .
$$

On désigne par $\mathbf{L L}_{\mathbb{Q}}^{\mathrm{tf}}\left(X_{\text {fét }}^{\mathbb{N}^{\circ}}, \breve{\mathfrak{o}}\right)$ la catégorie des $\breve{\mathfrak{o}}$-modules localement libres de type fini de $X_{\text {fét }}^{\mathbb{N}^{\circ}}$ à isogénie près. D'après (3.21.2), le foncteur (3.29.3) induit une équivalence de catégories

$$
\breve{\mu}_{\bar{x}, \mathbb{Q}}: \operatorname{Rep}_{\mathfrak{C}}^{\text {cont }}\left(\pi_{1}(X, \bar{x})\right) \stackrel{\sim}{\rightarrow} \mathbf{L L}_{\mathbb{Q}}^{\mathrm{tf}}\left(X_{\text {fét }}^{\mathbb{N}^{\circ}}, \breve{\mathfrak{o}}\right) .
$$

3.30. Soit $W$ un objet de $\operatorname{Rep}_{\mathfrak{o}}^{\text {ltf }}\left(\pi_{1}(X, \bar{x})\right)$ et $V$ une $\mathfrak{C}$-représentation continue de $\pi_{1}(X, \bar{x})$. Pour tout $i \geqslant 0$, on désigne par $\mathrm{H}_{\text {cont }}^{i}\left(\pi_{1}(X, \bar{x}), W\right)$ (resp. $\mathrm{H}_{\text {cont }}^{i}\left(\pi_{1}(X\right.$, $\bar{x}), V)$ ) le groupe de cohomologie continue de $\pi_{1}(X, \bar{x})$ à valeurs dans $W$ (resp. $V)$ (cf. [45, Section 2] ou [4, II.3.8]).

Posons $\mathbb{L}=\breve{\mu}_{\bar{x}}(W)$ (3.29.3). D'après (3.29.1), [4, II.3.10 et III.7.11], on a un isomorphisme canonique

$$
\mathrm{H}_{\text {cont }}^{i}\left(\pi_{1}(X, \bar{x}), W\right) \stackrel{\sim}{\rightarrow} \mathrm{H}^{i}\left(X_{\text {fét }}^{\mathbb{N}^{\circ}}, \mathbb{L}\right) .
$$

\section{Compléments de géométrie rigide}

4.1. Soit $\mathscr{S}=\operatorname{Spf}(\mathfrak{o})$ et $\mathfrak{X}$ un $\mathscr{S}$-schéma formel de présentation finie. Pour tout $\mathscr{O}_{\mathfrak{X}}$-module $\mathscr{F}$, on pose

$$
\mathscr{F}\left[\frac{1}{p}\right]=\mathscr{F} \otimes_{\mathbb{Z}_{p}} \mathbb{Q}_{p} .
$$

Celui-ci est canoniquement isomorphe à la clôture rigide de $\mathscr{F}$ [2, 2.10.1], [4, III.6.15]. On note $\operatorname{Mod}^{\mathrm{coh}}\left(\mathscr{O}_{\mathfrak{X}}\left[\frac{1}{p}\right]\right)$ la catégorie des $\mathscr{O}_{\mathfrak{X}}\left[\frac{1}{p}\right]$-modules cohérents et $\operatorname{Mod}_{\mathbb{Q}}^{\text {coh }}\left(\mathscr{O}_{\mathfrak{X}}\right)$ la catégorie des $\mathscr{O}_{\mathfrak{X}}$-modules cohérents à isogénie près. Alors le foncteur canonique

$$
\operatorname{Mod}^{\mathrm{coh}}\left(\mathscr{O}_{\mathfrak{X}}\right) \rightarrow \operatorname{Mod}^{\mathrm{coh}}\left(\mathscr{O}_{\mathfrak{X}}\left[\frac{1}{p}\right]\right), \quad \mathscr{F} \mapsto \mathscr{F}\left[\frac{1}{p}\right],
$$


induit une équivalence de catégories [4, III.6.16]

$$
\operatorname{Mod}_{\mathbb{Q}}^{\mathrm{coh}}\left(\mathscr{O}_{\mathfrak{X}}\right) \stackrel{\sim}{\rightarrow} \operatorname{Mod}^{\mathrm{coh}}\left(\mathscr{O}_{\mathfrak{X}}\left[\frac{1}{p}\right]\right) .
$$

4.2. On désigne par $\mathfrak{X}^{\text {rig }}$ l'espace rigide cohérent associé à $\mathfrak{X}[2,4.1 .6]$ et par $\mathfrak{X}_{\mathrm{ad}}^{\text {rig }}$ le topos admissible de $\mathfrak{X}^{\text {rig }}[2,4.4 .1]$. On pose $\Theta=\mathscr{S}^{\text {rig }}$. Pour tout $\mathscr{O}_{\mathfrak{X}}$-module $\mathscr{F}$, on peut lui associer un faisceau $\mathscr{F}^{\text {rig }}$ de $\mathfrak{X}_{\text {ad }}^{\text {rig }}[2,4.7 .4]$. Le faisceau $\left(\mathscr{O}_{\mathfrak{X}}\right)^{\text {rig }}$ est un anneau de $\mathfrak{X}_{\text {ad }}^{\text {rig }}$ que l'on note $\mathscr{O}_{\mathfrak{X}^{\text {rig }}}[2,4.7 .5]$. On a un morphisme canonique de topos annelés [2, (4.7.5.1)]

$$
\varrho_{\mathfrak{X}}:\left(\mathfrak{X}_{\text {ad }}^{\text {rig }}, \mathscr{O}_{\mathfrak{X}^{\text {rig }}}\right) \rightarrow\left(\mathfrak{X}_{\text {zar }}, \mathscr{O}_{\mathfrak{X}}\left[\frac{1}{p}\right]\right) .
$$

Celui-ci induit un morphisme canonique de topos annelés [2, (4.7.5.2)]

$$
\rho_{\mathfrak{X}}:\left(\mathfrak{X}_{\text {ad }}^{\text {rig }}, \mathscr{O}_{\mathfrak{X}^{\text {rig }}}\right) \rightarrow\left(\mathfrak{X}_{\text {zar }}, \mathscr{O}_{\mathfrak{X}}\right) .
$$

Pour tout $\mathscr{O}_{\mathfrak{X}}$-module cohérent $\mathscr{F}$, on a un isomorphisme canonique [2, 4.7.25]

$$
\rho_{\mathfrak{X}}^{*}(\mathscr{F}) \stackrel{\sim}{\rightarrow} \mathscr{F}^{\text {rig }} \text {. }
$$

4.3. Soit $V$ un $\mathfrak{C}$-schéma séparé de type fini. On peut lui associer un $\Theta$-espace rigide quasi séparé $V^{\text {an }}[2,7.4 .12]$. On désigne par $V_{\text {ad }}^{\text {an }}$ le topos admissible de $V^{\text {an }}[2,7.3 .4]$ et $\mathscr{O}_{V \text { an }}$ l'anneau structural de $V^{\text {an }}[2,7.3 .8]$. On a un morphisme de topos annelés (le morphisme de GAGA [2, (7.6.2.4)]) :

$$
\Phi_{V}:\left(V_{\text {ad }}^{\text {an }}, \mathscr{O}_{V \text { an }}\right) \rightarrow\left(V_{\text {zar }}, \mathscr{O}_{V}\right) .
$$

Si $F$ est un $\mathscr{O}_{V}$-module, on pose $F^{\text {an }}=\Phi_{V}^{*}(F)$ (l'image réciproque étant prise au sens des modules). L'anneau $\mathscr{O}_{V^{\text {an }}}$ est cohérent (cf. [2, 7.3.10 et 7.3.11]). On désigne par $\operatorname{Mod}^{\mathrm{coh}}\left(\mathscr{O}_{V}\right)\left(\right.$ resp. $\left.\operatorname{Mod}^{\mathrm{coh}}\left(\mathscr{O}_{V \text { an }}\right)\right)$ la catégorie des $\mathscr{O}_{V}$-modules (resp.

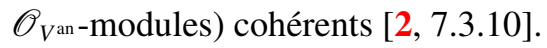

4.4. Soit $X$ un $\check{\bar{S}}$-schéma séparé et de présentation finie (2.1), $\widehat{X}$ le schéma formel complété $p$-adique de $X$ et $V=X \otimes_{\mathfrak{o}} \mathfrak{C}$. On a un $\Theta$-morphisme canonique [2, 7.4.12.2]

$$
\widehat{X}^{\mathrm{rig}} \rightarrow V^{\mathrm{an}} \text {. }
$$

Si $X$ est propre sur $\check{\bar{S}}$, celui-ci est un isomorphisme et, par suite, $V^{\text {an }}$ est cohérent $[2,7.4 .14]$. Soit $\mathscr{F}$ un $\mathscr{O}_{X}$-module cohérent et $\widehat{\mathscr{F}}$ son complété $p$-adique. On a un isomorphisme fonctoriel $[2,7.6 .7]$

$$
\widehat{\mathscr{F}}^{\mathrm{rig}} \stackrel{\sim}{\rightarrow}(\mathscr{F} \mid V)^{\mathrm{an}} \mid \widehat{X}^{\mathrm{rig}} .
$$


Proposition 4.5. Soit $V$ un $\mathfrak{C}$-schéma séparé de type fini et $F$ un $\mathscr{O}_{V}$-module cohérent. Si $F^{\text {an }}=0, F$ est également nul.

Preuve. Le support de $F$ est un sous-ensemble fermé de $V$. Comme chaque sousensemble fermé de $V$ a un point fermé, il suffit de démontrer que, pour tout point fermé $P$ de $V, F_{P}$ est nul.

Soit $P$ un $\mathfrak{C}^{\prime}$-point de $V$ et $Q: \Theta \rightarrow V^{\text {an }}$ le morphisme d'espaces rigides associé $[2,7.4 .12]$. D'après $[2,7.4 .12 .3]$, il existe un $\check{\bar{S}}$-schéma $X$ séparé de présentation finie et de fibre générique $V$ tel que $Q$ se factorise en un morphisme $\Theta \rightarrow \widehat{X}^{\text {rig }}$ que l'on note encore $Q$, suivi du morphisme canonique $\widehat{X}^{\text {rig }} \rightarrow V^{\text {an }}$ (4.4.1). D'après $[2,3.3 .12$ et 4.1 .20$]$, il existe un unique point rigide $\mathscr{Q}: \mathscr{S} \rightarrow \widehat{X}[2,3.3 .1]$ tel que $Q=\mathscr{Q}^{\text {rig }}$.

Pour démontrer que $F_{P}$ est nul, on peut se ramener au cas où $X=\operatorname{Spec}(A)$ est affine. On note $\widehat{A}$ le complété $p$-adique de $A$ et on pose $B=A\left[\frac{1}{p}\right], B^{\prime}=\widehat{A}\left[\frac{1}{p}\right]$ et $f: B \rightarrow B^{\prime}$ le morphisme canonique. Le point rigide $\mathscr{Q}$ de $\widehat{X}$ correspond à un idéal maximal $\mathfrak{q}$ de $B^{\prime}$ (cf. [2, 3.3.2]). D'après [2, 1.12.11(i)], $\mathfrak{p}=f^{-1}(\mathfrak{q})$ est l'idéal maximal de $B$ correspondant au point fermé $P$.

Il existe un $\mathscr{O}_{X}$-module cohérent $\mathscr{F}$ tel que $\mathscr{F} \otimes_{\mathfrak{o}} \mathfrak{C} \simeq F[2,2.6 .23]$. En vertu de (4.4.2), on a un isomorphisme $\widehat{\mathscr{F}}^{\text {rig }} \stackrel{\sim}{\rightarrow} F^{\text {an }} \mid \widehat{X}^{\text {rig }}$. On en déduit que $\widehat{\mathscr{F}}^{\text {rig }}=0$. Il existe un $A$-module cohérent $M$ tel que $\mathscr{F}$ soit isomorphe au $\mathscr{O}_{X}$-module associé à $M$. Notons $\widehat{M}$ le complété $p$-adique de $M$. D'après $[2,1.12 .16]$, le couple $(A$, $p A)$ vérifie la condition de Krull $[2,1.8 .25]$ et on a alors un isomorphisme

$$
M \otimes_{A} \widehat{A} \stackrel{\sim}{\rightarrow} \widehat{M} .
$$

D'après $[2,4.8 .24]$, le $B^{\prime}$-module $\widehat{M}\left[\frac{1}{p}\right]$ est nul. On en déduit que

$$
\left(M\left[\frac{1}{p}\right]\right)_{\mathfrak{p}} \otimes_{B_{\mathfrak{p}}} B_{\mathfrak{q}}^{\prime} \simeq\left(\widehat{M}\left[\frac{1}{p}\right]\right)_{\mathfrak{q}}
$$

est nul. D'après [2, 1.12.17], le morphisme canonique $A \rightarrow \widehat{A}$ est plat. Il en est de même du morphisme local $B_{\mathfrak{p}} \rightarrow B_{\mathfrak{q}}^{\prime}$. En vertu de [26, 6.6.1], $B_{\mathfrak{q}}^{\prime}$ est fidèlement plat sur $B_{\mathfrak{p}}$. Le $B_{\mathfrak{p}}$-module $\left(M\left[\frac{1}{p}\right]\right)_{\mathfrak{p}} \simeq F_{P}$ est alors nul, d'où la proposition.

Corollaire 4.6. Sous les hypothèses de 4.5, le foncteur

$$
\Phi_{V}^{*}: \operatorname{Mod}^{\mathrm{coh}}\left(\mathscr{O}_{V}\right) \rightarrow \operatorname{Mod}^{\mathrm{coh}}\left(\mathscr{O}_{V} \text { an }\right) \quad F \mapsto F^{\mathrm{an}}
$$

est exact et fidèle.

Preuve. Cela résulte de 4.5 et $[2,7.6 .8]$. 
Proposition 4.7. Soit $V$ un schéma séparé de type fini sur $\mathfrak{C}$ et $F$ un $\mathscr{O}_{X^{-}}$ module cohérent. Pour que $F$ soit plat, il faut et il suffit que $F^{\text {an }}$ soit un $\mathscr{O}_{V^{\text {an }}-}$ module plat.

Preuve. Si $F$ est plat, c'est alors un fibré vectoriel sur $V$. On en déduit que $F^{\text {an }}$ est localement libre de type fini et qu'il est plat.

Supposons que $F^{\text {an }}$ soit plat. Soit $U$ un ouvert de $V, F_{U}$ la restriction de $F$ à $U$ et $F_{U}^{\text {an }}=\Phi_{U}^{*}(F)$ (4.6.1). Le $\mathscr{O}_{U}$ an -module $F_{U}^{\text {an }}$ s'identifie à la restriction de $F^{\text {an }}$ sur $U^{\text {an }}$. Comme $U_{\text {ad }}^{\text {an }}$ a suffisamment de points [2, 7.3.14], $F_{U}^{\text {an }}$ est un $\mathscr{O}_{U^{\text {an }} \text {-module }}$ plat. Comme le foncteur $\Phi_{U}^{*}$ (4.6.1) est exact et fidèle, on en déduit que le foncteur

$$
F_{U} \otimes_{\mathscr{O}_{U}}-: \operatorname{Mod}^{\mathrm{coh}}\left(\mathscr{O}_{U}\right) \rightarrow \operatorname{Mod}^{\mathrm{coh}}\left(\mathscr{O}_{U}\right)
$$

est exact. La proposition s'ensuit compte tenu de [2, 1.3.17].

\section{Rappel sur les courbes}

5.1. Dans cet article, une courbe sur un corps $F$ est un schéma séparé de type fini et géométriquement connexe sur $F$ de dimension 1. Une courbe propre sur un corps algébriquement clos est dite semi-stable si elle est réduite et a pour seules singularités des points doubles ordinaires. On notera que cette définition est moins restrictive que la définition classique puisque l'on n'impose aucune condition sur l'intersection avec les composantes rationnelles.

5.2. Soit $T=\operatorname{Spec}(R)$ le spectre d'un anneau de valuation $R$ de hauteur 1 , de corps des fractions $F$, et $\bar{F}$ une clôture algébrique de $F$. On note $\tau$ (resp. $t$ ) le point générique (resp. fermé) de $T$ et $\bar{\tau}$ le point géométrique générique correspondant à $\bar{F}$.

Une $T$-courbe est un $T$-schéma plat, séparé, de présentation finie et de dimension relative 1 dont la fibre générique est une courbe lisse sur $F$. Une $T$ courbe $f: X \rightarrow T$ est propre (resp. régulière, resp. normale) si $f$ est propre (resp. $X$ est régulier, resp. $X$ est normal). D'après [31, Section 4, 3.8], toute $T$ courbe est irréductible et réduite. D'après la factorisation de Stein [24, 4.3.1], la fibre spéciale d'une $T$-courbe propre est géométriquement connexe et est donc une $t$-courbe propre (5.1). On dit qu'une $T$-courbe $X_{1}$ domine une autre $T$-courbe $X_{2}$ s'il existe un $T$-morphisme dominant de $X_{1}$ dans $X_{2}$.

On dit qu'une $T$-courbe est semi-stable si elle est propre et si sa fibre spéciale géométrique est une courbe semi-stable. On dit qu'une $T$-courbe $X$ est cohomologiquement plate en dimension 0 si elle est propre et si le faisceau $\mathscr{O}_{X}$ est cohomologiquement plat sur $T$ en dimension 0 , i.e. si le morphisme structural $\lambda$ : $X \rightarrow T$ induit un isomorphisme $\mathscr{O}_{T} \stackrel{\sim}{\rightarrow} \lambda_{*}\left(\mathscr{O}_{X}\right)$ universellement [24, 7.8.1]. On 
notera qu'une $T$-courbe propre à fibres géométriquement réduites (en particulier une $T$-courbe semi-stable) est cohomologiquement plate en dimension 0 (cf. [24, 7.8.6]).

Une $T$-courbe propre $X$ est appelée modèle (resp. $T$-modèle) de sa fibre générique (resp. de sa fibre géométrique générique). Soit $C$ une courbe propre et lisse sur $\bar{F}$. On dit qu'un $T$-modèle $X_{1}$ de $C$ domine un autre $T$-modèle $X_{2}$ de $C$ s'il existe un $T$-morphisme de $X_{1}$ dans $X_{2}$ induisant un isomorphisme entre les fibres génériques.

5.3. Pour toute $T$-courbe semi-stable $X$, le foncteur de Picard relatif $\mathrm{Pic}_{X / T}^{0}(2.7)$ est représentable par un $T$-schéma semi-abélien (cf. [7, 9.4.1]). Si $T=\check{\bar{S}}(2.1)$, on a $\operatorname{Pic}(\stackrel{\check{S}}{)})=0$ et donc un isomorphisme canonique $\operatorname{Pic}(X) \stackrel{\sim}{\rightarrow} \operatorname{Pic}_{X / \overline{\bar{S}}}(\stackrel{\bar{S}}{)})$ (cf. [7, 8.1, Prop. 4]).

DÉFINITION 5.4. Soit $(T, \tau)$ comme dans 5.2 et $X$ une $T$-courbe propre.

(i) On appelle $\tau$-revêtement de $X$ la donnée d'un $T$-morphisme propre de présentation finie de $T$-courbes $\varphi: Y \rightarrow X$ tel que le morphisme $\varphi_{\tau}: Y_{\tau} \rightarrow X_{\tau}$ soit un revêtement étale.

(ii) Soit $\varphi: Y \rightarrow X$ un $\tau$-revêtement. Si la $T$-courbe propre $Y$ est cohomologiquement plate en dimension 0 (resp. a des fibres géométriquement réduites, resp. est semi-stable, resp. est régulière), on dit que $\varphi: Y \rightarrow X$ est un $\tau$-revêtement cohomologiquement plat en dimension 0 (resp. à fibres géométriquement réduites, resp. semi-stable, resp. régulier) et en abrégé un $\tau$-revêtement c.p.d.0.

(iii) On dit qu'un $\tau$-revêtement $\varphi: Y \rightarrow X$ est fini si le morphisme sous-jacent $Y \rightarrow X$ est fini.

(iv) Soit $\varphi: Y \rightarrow X$ un $\tau$-revêtement. Si $\varphi_{\tau}: Y_{\tau} \rightarrow X_{\tau}$ est un revêtement étale et galoisien de groupe des automorphismes $G$ et si l'action de $G$ sur $Y_{\tau}$ s'étend en une $X$-action sur $Y$, on dit que $\varphi: Y \rightarrow X$ est un $\tau$-revêtement galoisien.

Soit $\varphi_{1}: Y_{1} \rightarrow X$ et $\varphi_{2}: Y_{2} \rightarrow X$ deux $\tau$-revêtements de $X$. Un morphisme de $\varphi_{1}$ dans $\varphi_{2}$ est un $X$-morphisme $\pi: Y_{1} \rightarrow Y_{2}$. On notera alors que $Y_{1}$ domine $Y_{2}$ et on dira que $\varphi_{1}$ domine $\varphi_{2}$. Supposons de plus que $\varphi_{1}$ et $\varphi_{2}$ soient galoisiens. Alors, le morphisme $\pi_{\tau}: Y_{1, \tau} \rightarrow Y_{2, \tau}$ est un revêtement galoisien. Notons $u$ : $\operatorname{Aut}\left(Y_{1, \tau} / X_{\tau}\right) \rightarrow \operatorname{Aut}\left(Y_{2, \tau} / X_{\tau}\right)$ l'homomorphisme induit par $\pi_{\tau}$. On dit que $\pi$ est équivariant s'il est $u$-équivariant. 
THÉORÈME 5.5 (Grothendieck; cf. [1] pour $g(C) \geqslant 2$ et [31], Section 10.2, pour $g(C)=1)$. Pour toute $\bar{K}$-courbe propre et lisse $C(2.1)$, il existe un trait $S^{\prime}$ fini sur $S$ tel que $C$ admette un $S^{\prime}$-modèle semi-stable.

5.6. Soit $X$ une $S$-courbe propre. Une désingularisation de $X$ est un modèle régulier de $X_{\eta}$ dominant $X$. On sait qu'il existe une désingularisation de $X$ (cf. [31, Section 8, 3.44]). Si le genre de $X_{\eta}$ est $\geqslant 1$, grâce au critère de contraction de Castelnuovo (cf. [31, Section 9, 3.21]), on peut construire une désingularisation minimale $Y$ de $X$ unique à isomorphisme près, c'est-à-dire que toute désingularisation $Y^{\prime} \rightarrow X$ se factorise d'une façon unique à travers $Y$. D'après [31, Section 9,3.13], tout $\eta$-automorphisme de $X_{\eta}$ s'étend en un $S$ automorphisme de la désingularisation minimale de $X$.

Proposition 5.7 [32, 2.7 et 2.8]. Soit X une S-courbe propre. Il existe un trait $S^{\prime}$ fini sur $S$ tel que $X_{S^{\prime}}$ soit dominé par un $S^{\prime}$-modèle semi-stable et régulier $X^{\prime}$ de $X_{\bar{\eta}}$. Si le genre de $X_{\eta}$ est $\geqslant 1$, on peut trouver $S^{\prime}$ de sorte que $X^{\prime}$ soit la désingularisation minimale de $X_{S^{\prime}}$.

Corollaire 5.8. Soit $C$ une $\bar{K}$-courbe propre et lisse, $X_{1}$ et $X_{2}$ deux $S$-modèles de $C$. Il existe un trait $S^{\prime}$ fini sur $S$ et un $S^{\prime}$-modèle $X_{3}$ semi-stable et régulier de $C$ dominant $X_{1, S^{\prime}}$ et $X_{2, S^{\prime}}$.

Preuve. Il existe un $S$-modèle $X$ de $C$ dominant $X_{1}$ et $X_{2}$ [12, prop. 20]. L'assertion résulte alors de 5.7.

5.9. On dit qu'un $S$-schéma $X$ a une réduction semi-stable si, localement pour la topologie étale, $X$ est isomorphe à un $S$-schéma de la forme

$$
\operatorname{Spec}\left(\mathcal{O}_{K}\left[t_{1}, \ldots, t_{m}\right] /\left(t_{1} \ldots t_{n}-\pi\right)\right),
$$

où $\pi$ est une uniformisante de $\mathcal{O}_{K}$, et $m$ et $n$ sont des entiers vérifiant $m \geqslant$ $n \geqslant 1$. On notera qu'une $S$-courbe propre admet une réduction semi-stable si et seulement si elle est semi-stable et régulière (cf. $[40,1.6])$.

5.10. Soit $X$ une $S$-courbe à fibres géométriquement réduites. D'après [31, Section 4, 1.18], pour tout trait $S^{\prime}$ fini sur $S$, le schéma $X_{S^{\prime}}$ est normal. On en déduit par passage à la limite projective que le schéma $\bar{X}=X \times{ }_{S} \bar{S}(2.1 .1)$ est normal.

Proposition 5.11. Soit $X$ une $S$-courbe propre.

(i) Étant donné un nombre fini de $\eta$-revêtements (resp. $\eta$-revêtements galoisiens) 
$\varphi_{i}: Y_{i} \rightarrow X$, il existe un trait $\left(S^{\prime}, \eta^{\prime}\right)$ fini sur $(S, \eta)$, un $\eta^{\prime}$-revêtement semistable, régulier et galoisien $\varphi: Y \rightarrow X_{S^{\prime}}$ et pour chaque $\varphi_{i}$, un morphisme (resp. un morphisme équivariant) de $\varphi$ dans $\varphi_{i} \times{ }_{S} S^{\prime}$.

(ii) Pour tout $\eta$-revêtement fini (resp. fini et galoisien) $\varphi: Y \rightarrow X$, il existe un trait $\left(S^{\prime}, \eta^{\prime}\right)$ fini sur $(S, \eta)$ tel que $\varphi \times{ }_{S} S^{\prime}$ soit dominé par un $\eta^{\prime}$ revêtement fini, à fibres géométriquement réduites (resp. fini, galoisien, à fibres géométriquement réduites) de $X_{S^{\prime}}$.

(iii) Pour tout $\eta$-revêtement $\varphi: Y \rightarrow X$, il existe un trait $\left(S^{\prime}, \eta^{\prime}\right)$ fini $\operatorname{sur}(S, \eta)$, un $S^{\prime}$-modèle semi-stable $X^{\prime}$ de $X_{\bar{\eta}}$ dominant $X_{S^{\prime}}$ et un $\eta^{\prime}$-revêtement semistable, régulier, fini et galoisien $\varphi^{\prime}: Y^{\prime} \rightarrow X^{\prime}$ qui s'insère dans un diagramme commutatif

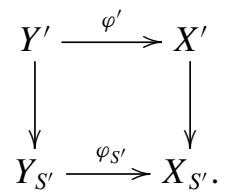

Preuve. (i) Si le genre de $X_{\eta}$ est nul, $X_{\bar{\eta}}$ n'admet aucun revêtement étale non trivial. Dans ce cas, l'énoncé (i) résulte de 5.7 appliqué aux produits des $Y_{i}$ au-dessus de $X$. Pour $g\left(X_{\eta}\right) \geqslant 1$, cela résulte de 5.7 et des propriétés de la désingularisation minimale, appliquées au produit des $Y_{i}$ au-dessus de $X$ (cf. [12, Thm. 12] et [13, Thm. 4]).

(ii) D'après [14, Thm. 2.0], il existe un trait $\left(S^{\prime}, \eta^{\prime}\right)$ fini sur $(S, \eta)$ tel que la normalisation $Y^{\prime}$ de $Y_{S^{\prime}}$ ait une fibre spéciale géométriquement réduite. Comme $Y$ est excellent, $Y^{\prime}$ est fini sur $Y$. Supposons de plus que $\varphi: Y \rightarrow X$ soit galoisien. Comme $Y^{\prime}$ est la clôture intégrale de $Y$ dans $Y \times{ }_{s} \eta^{\prime}$, l'action de $\operatorname{Aut}\left(Y_{\eta} / X_{\eta}\right) \operatorname{sur} Y$ s'étend en une action sur $Y^{\prime}$, i.e. $Y^{\prime} \rightarrow X_{S^{\prime}}$ est aussi un $\eta^{\prime}$-revêtement galoisien.

(iii) D'après (i), quitte à remplacer $K$ par une extension finie, $\varphi$ est dominé par un $\eta$-revêtement semi-stable, régulier et galoisien $Y^{\prime} \rightarrow X$. Notons $G=$ $\operatorname{Aut}\left(Y_{\eta}^{\prime} / X_{\eta}\right)$. L'action de $G$ sur $Y_{\eta}^{\prime}$ s'étend en une $X$-action sur $Y^{\prime}$. Comme $Y^{\prime}$ est projective [30, Thm. 2.8], le quotient $Y^{\prime} / G$ existe [23, V.1]; notons-le $X^{\prime}$. Calquant la démonstration de $[32,3.8]$ (cf. aussi [38, Prop. 5]), on vérifie que $X^{\prime}$ est un modèle semi-stable de $X_{\eta}$ dominant $X$. Le morphisme $Y^{\prime} \rightarrow X^{\prime}$ est donc un $\eta$-revêtement semi-stable, régulier, fini et galoisien. Comme le diagramme

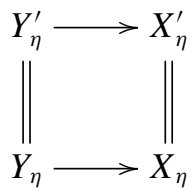


est commutatif, il en est de même de (5.11.1) en vertu de [26, 8.2.2.1].

Corollaire 5.12. Soit X une $\bar{S}$-courbe propre.

(i) Étant donné un nombre fini de $\bar{\eta}$-revêtements de $X$, il existe un $\bar{\eta}$-revêtement semi-stable et galoisien de $X$ qui les domine tous.

(ii) Tout $\bar{\eta}$-revêtement $\varphi: Y \rightarrow X$ fini et galoisien est dominé par un $\bar{\eta}$ revêtement fini, galoisien et à fibres géométriquement réduites.

Preuve. D'après la théorie de la descente [25, 8.8.3 et 8.10.5], quitte à remplacer $S$ par une extension finie, tout $\bar{\eta}$-revêtement (resp. $\bar{\eta}$-revêtement fini, resp. $\bar{\eta}$ revêtement fini et galoisien) de $X$ se descend en un $\eta$-revêtement (resp. $\eta$ revêtement fini, resp. $\eta$-revêtement fini et galoisien). Le corollaire résulte alors de 5.11 .

5.13. Soit $X$ une $\stackrel{\check{S}}{S}$-courbe projective et de présentation finie et $C=X_{\check{\eta}}$ (2.1). L'anneau $\mathfrak{o}$ est universellement cohérent [2, 1.12.15]. Par suite, l'anneau $\mathscr{O}_{X}$ est cohérent. On note $\operatorname{Mod}^{\mathrm{coh}}\left(\mathscr{O}_{X}\right)$ (resp. $\operatorname{Mod}^{\mathrm{coh}}\left(\mathscr{O}_{C}\right)$ ) la catégorie des $\mathscr{O}_{X}$-modules (resp. $\mathscr{O}_{C}$-modules) cohérents et $\operatorname{Mod}_{\mathscr{Q}}^{\text {coh }}\left(\mathscr{O}_{X}\right)$ la catégorie de $\mathscr{O}_{X^{-}}$ modules cohérents à isogénie près. D'après [2, 2.6.23], tout $\mathscr{O}_{C}$-module cohérent se prolonge en un $\mathscr{O}_{X}$-module cohérent. Étant donné deux objets $\mathcal{F}$ et $\mathcal{F}^{\prime}$ de $\operatorname{Mod}^{\mathrm{coh}}\left(\mathscr{O}_{X}\right)$, on a un isomorphisme

$$
\operatorname{Hom}_{\mathscr{O}_{X}}\left(\mathcal{F}, \mathcal{F}^{\prime}\right) \otimes_{\mathbb{Z}} \mathbb{Q} \stackrel{\sim}{\rightarrow} \operatorname{Hom}_{\mathscr{O}_{C}}\left(\mathcal{F}_{\bar{\eta}}, \mathcal{F}_{\bar{\eta}}^{\prime}\right) .
$$

Celui-là implique que $\mathcal{F}$ et $\mathcal{F}^{\prime}$ sont isogènes dans $\operatorname{Mod}^{\text {coh }}\left(\mathscr{O}_{X}\right)$ si et seulement si leurs fibres génériques sont isomorphes sur $C$. Le foncteur canonique $\operatorname{Mod}^{\text {coh }}\left(\mathscr{O}_{X}\right) \rightarrow \operatorname{Mod}^{\text {coh }}\left(\mathscr{O}_{C}\right)$ induit alors une équivalence de catégories

$$
\operatorname{Mod}_{\mathbb{Q}}^{\mathrm{coh}}\left(\mathscr{O}_{X}\right) \stackrel{\sim}{\rightarrow} \operatorname{Mod}^{\mathrm{coh}}\left(\mathscr{O}_{C}\right)
$$

5.14. Conservons les notations de 5.13. On pose $\mathscr{S}=\operatorname{Spf}(\mathfrak{o})$ et on note $\mathfrak{X}$ le schéma formel complété $p$-adique de $X$ qui est propre et de présentation finie $\operatorname{sur} \mathscr{S}$. En particulier, $\mathscr{O}_{\mathfrak{X}}$ est cohérent $\left[2,2.8\right.$.1]. Pour tout $\mathscr{O}_{X}$-module $\mathcal{F}$, on note $\widehat{\mathcal{F}}$ son complété $p$-adique. On note $\operatorname{Mod}^{\text {coh }}\left(\mathscr{O}_{\mathfrak{X}}\right)$ la catégorie des $\mathscr{O}_{\mathfrak{X}}$-modules cohérents. Comme $X$ est projectif et de présentation finie sur $\check{\bar{S}}$, le foncteur

$$
\operatorname{Mod}^{\mathrm{coh}}\left(\mathscr{O}_{X}\right) \rightarrow \operatorname{Mod}^{\mathrm{coh}}\left(\mathscr{O}_{\mathfrak{X}}\right), \quad \mathcal{F} \mapsto \widehat{\mathcal{F}}
$$

induit une équivalence de catégories [2, 2.13.8]. 
Choisissons des quasi-inverses de (4.1.2) et (5.14.1). On désigne par $J_{\mathfrak{X}}$ le foncteur composé de l'équivalence de catégories (5.13.2) et des quasi-inverses de (4.1.2), (5.14.1)

$$
J \mathfrak{X}: \operatorname{Mod}^{\operatorname{coh}}\left(\mathscr{O}_{\mathfrak{X}}\left[\frac{1}{p}\right]\right) \stackrel{\sim}{\rightarrow} \operatorname{Mod}^{\mathrm{coh}}\left(\mathscr{O}_{C}\right) .
$$

LEMME 5.15. Conservons les notations de 5.14. Pour tout $\mathscr{O}_{\mathfrak{X}}\left[\frac{1}{p}\right]$-module localement projectif de type fini $\mathscr{F}(2.9), J_{\mathfrak{X}}(\mathscr{F})$ (5.14.2) est un fibré vectoriel $\operatorname{sur} C$.

Preuve. Posons $F=J \mathfrak{X}(\mathscr{F})$. On désigne par $\mathfrak{X}^{\text {rig }}$ l'espace rigide cohérent associé au schéma formel $\mathfrak{X}(4.2)$ et $C^{\text {an }}$ l'espace rigide quasi séparé associé au $\mathfrak{C}$-schéma propre $C$ (4.3). On a un isomorphisme $\mathfrak{X}^{\text {rig }} \stackrel{\sim}{\rightarrow} C^{\text {an }}(4.4)$ et un morphisme de topos annelés $\varrho_{\mathfrak{X}}:\left(\mathfrak{X}_{\text {rig }}^{\text {an }}, \mathscr{O}_{\mathfrak{X}_{\text {rig }}}\right) \rightarrow\left(\mathfrak{X}_{\text {zar }}, \mathscr{O}_{\mathfrak{X}}\left[\frac{1}{p}\right]\right)$ (4.2.1). En vertu de (4.2.2) et (4.4.2), on a un isomorphisme

$$
\varrho_{\mathfrak{X}}^{*}(\mathscr{F}) \stackrel{\sim}{\rightarrow} F^{\text {an }} .
$$

Le $\mathscr{O}_{C^{\text {an }}}$-module $F^{\text {an }}$ est alors localement projectif de type fini. Par suite, c'est un

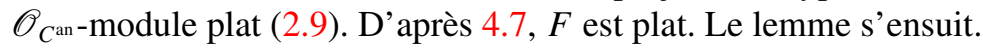

LEMME 5.16. Conservons les notations de 5.14.

(i) Pour tout $\mathscr{O}_{\mathfrak{X}}$-module $\mathscr{H}$, on a un isomorphisme canonique

$$
\Gamma\left(\mathfrak{X}, \mathscr{H}\left[\frac{1}{p}\right]\right) \simeq \Gamma(\mathfrak{X}, \mathscr{H}) \otimes_{\mathbb{Z}} \mathbb{Q} .
$$

(ii) Soit $\mathscr{F}$ un $\mathscr{O}_{\mathfrak{X}}\left[\frac{1}{p}\right]$-module cohérent et $F=J \mathfrak{X}(\mathscr{F})$. Pour tout entier $i \geqslant 0$, on a un isomorphisme canonique

$$
\mathrm{H}^{i}(\mathfrak{X}, \mathscr{F}) \simeq \mathrm{H}^{i}(C, F) .
$$

Preuve. (i) Par platitude de $\mathscr{O}_{\mathfrak{X}}$ sur o, il suffit de démontrer l'isomorphisme (5.16.1) pour les ouverts affines. Dans ce cas, cela résulte de [2, 2.10.5].

(ii) Il existe un $\mathscr{O}_{X}$-module cohérent $\mathcal{F}$ tel que $\mathscr{F} \simeq \widehat{\mathcal{F}}\left[\frac{1}{p}\right]$ et que $F \simeq \mathcal{F}_{\bar{\eta}}$. D'après [2, 2.13.2], on a un isomorphisme canonique

$$
\mathrm{H}^{i}(X, \mathcal{F}) \simeq \mathrm{H}^{i}(\mathfrak{X}, \widehat{\mathcal{F}}) .
$$

On déduit par (i) un isomorphisme $H^{i}(\mathfrak{X}, \widehat{\mathcal{F}}) \otimes_{\mathbb{Z}} \mathbb{Q} \simeq \mathrm{H}^{i}(\mathfrak{X}, \mathscr{F})$. Par platitude, on a un isomorphisme canonique

$$
\mathrm{H}^{i}(X, \mathcal{F}) \otimes_{\mathbb{Z}} \mathbb{Q} \simeq \mathrm{H}^{i}(C, F) .
$$

L'assertion s'ensuit. 


\section{Correspondance de Deninger-Werner}

On rappelle que le corps résiduel $k$ de $\mathcal{O}_{K}$ est une clôture algébrique de $\mathbb{F}_{p}$ (2.1).

DÉfinition 6.1. (i) Soit $C$ une courbe propre et lisse sur un corps algébriquement clos de caractéristique $p$ et $\operatorname{Fr}_{C}: C \rightarrow C$ le Frobenius absolu de $C$. On dit qu'un fibré vectoriel $F$ sur $C$ est fortement semi-stable si $\left(\operatorname{Fr}_{C}^{n}\right)^{*}(F)$ est un fibré vectoriel semi-stable pour tout entier $n \geqslant 0$.

(ii) Soit $C$ une courbe propre sur un corps algébriquement clos de caractéristique $p$ et $\pi: \widetilde{C} \rightarrow C$ la normalisation du sous-schéma réduit sous-jacent à $C$. On dit qu'un fibré vectoriel $F$ sur $C$ est fortement semi-stable de degré 0 si $\pi^{*}(F)$ est fortement semi-stable de degré 0 sur chaque composante irréductible de $\widetilde{C}$.

On notera qu'un fibré vectoriel semi-stable sur une courbe propre et lisse de genre $g \geqslant 2$ sur un corps de caractéristique $p$ n'est pas nécessairement fortement semi-stable (cf. [21]).

ThÉORÈme 6.2 [13, Thm. 16, Thm. 17] et [46, 2.4]. Soit $X$ une $\bar{S}$-courbe propre, $\check{X}=X \times{ }_{\bar{S}} \check{\bar{S}}(2.1)$ et $\mathcal{F}$ un fibré vectoriel sur $\check{X}$. Les conditions suivantes sont équivalentes.

(i) La fibre spéciale $\mathcal{F}_{s}$ de $\mathcal{F}$ est fortement semi-stable de degré 0 sur $X_{s}$.

(ii) Pour tout $n \geqslant 1$, il existe un $\bar{\eta}$-revêtement $\varphi: X^{\prime} \rightarrow X(5.4)$ tel que $\varphi_{n}^{*}\left(\mathcal{F}_{n}\right)$ soit libre de type fini, où $\mathcal{F}_{n}$ (resp. $\varphi_{n}$ ) est la réduction modulo $p^{n}$ de $\mathcal{F}$ (resp. $\varphi$ ) (2.1.1).

(iii) Il existe un entier $n \geqslant 1$ et un $\bar{\eta}$-revêtement $\varphi: X^{\prime} \rightarrow X$ tels que $\varphi_{n}^{*}\left(\mathcal{F}_{n}\right)$ soit libre de type fini.

DÉfINITION 6.3. Conservons les notations de 6.2. On appelle fibré vectoriel de Deninger-Werner sur $\check{X}$ tout fibré vectoriel sur $\check{X}$ vérifiant les conditions équivalentes de 6.2. On désigne par $\mathfrak{V}_{\check{X}}^{\mathrm{DW}}$ la sous-catégorie pleine de $\operatorname{Vect}_{\check{X}}$ (2.8) formée des fibrés vectoriels de Deninger-Werner.

Proposition 6.4. Soit $X$ une $\bar{S}$-courbe semi-stable. Un fibré en droites sur $\check{X}$ est de Deninger-Werner si et seulement si sa classe appartient à $\operatorname{Pic}_{\check{X} / \bar{S}}^{0}(\check{\bar{S}})(2.7)$. 
Preuve. D'après [13, Thm. 12], la catégorie $\mathfrak{V}_{\check{X}}^{\mathrm{DW}}$ contient tous les fibrés en droites dont les classes sont dans $\operatorname{Pic}_{\check{X} / \bar{S}}^{0} \stackrel{\check{\bar{S}}}{0}$. Inversement, pour tout fibré en droites $L \in \mathfrak{V}_{\check{X}}^{\mathrm{DW}}$, l'image inverse de la fibre spéciale $L_{s}$ sur la normalisation de chaque composante irréductible de $X_{s}$ est de degré 0. D'après [13, Thm.13], la fibre générique $L_{\check{\bar{\eta}}}$ de $L$ est donc aussi de degré 0 . Ceci implique que $L \in \operatorname{Pic}_{\check{X} / \check{\bar{S}}}^{0}(\check{\bar{S}})$ en vertu de [7, 9.1.13].

Proposition 6.5. Soit $X$ une $\bar{S}$-courbe propre et lisse, $n$ un entier $\geqslant 1$ et $L$ un fibré en droites dont la classe appartient à $\mathrm{Pic}_{\check{X} / \overline{\bar{S}}}^{0}\left(\frac{\check{S}}{\bar{S}}\right)$. Alors, il existe un $\bar{\eta}$ revêtement fini, galoisien et à fibres géométriquement réduites de $X$ trivialisant $L_{n}$.

Preuve. Quitte à remplacer $K$ par une extension finie, il existe une $S$-courbe propre et lisse $Y$ telle que $X \simeq \bar{Y}$ et un point $x \in Y(S)$. On sait que $\operatorname{Pic}_{Y / S}^{0}$ est un schéma abélien sur $S$ [7, 9.4.4]. La classe de $L$ est contenue dans $\operatorname{Pic}_{Y / S}^{0}(\stackrel{\bar{S}}{\bar{S}})$. On a un morphisme de $S$-schémas

$$
j_{x}: Y \rightarrow \operatorname{Pic}_{Y / S}^{0}
$$

défini, pour tout $S$-schéma $T$, par

$$
Y(T) \rightarrow \operatorname{Pic}_{Y / S}^{0}(T) \quad y \mapsto\left[\mathscr{O}_{Y_{T}}(y-x)\right] .
$$

Notons $A=\left(\operatorname{Pic}_{Y / S}^{0}\right)^{\vee}$ le schéma abélien dual de $\operatorname{Pic}_{Y / S}^{0}$ et considérons le morphisme d'Albanese défini par $x \in Y(S)$,

$$
\varphi_{x}: Y \stackrel{j_{x}}{\rightarrow} \operatorname{Pic}_{Y / S}^{0} \stackrel{\Phi}{\rightarrow} A
$$

où $\Phi$ est la polarisation canonique [34, 2.6.4]. D'après [34, 2.7.9], le morphisme

$$
A^{\vee} \simeq \operatorname{Pic}_{A / S}^{0} \stackrel{\varphi_{x}^{*}}{\rightarrow} \operatorname{Pic}_{Y / S}^{0} \simeq A^{\vee},
$$

induit par $\varphi_{x}$, s'identifie à $-\mathrm{id}_{A^{\vee}}$. Il existe donc un fibré en droites $L^{\prime} \operatorname{sur} \check{\check{A}}=$ $A \times{ }_{S} \check{S}_{S}$ dont la classe appartient à $\operatorname{Pic}_{A / S}^{0}(\check{\bar{S}})$ tel que $\varphi_{x}^{*}\left(L^{\prime}\right) \simeq L$. Par descente [25, 8.8.3], le groupe $\operatorname{Pic}_{A / S}(k) \simeq \operatorname{Pic}_{A_{s} / k}(k)$ est de torsion. Il existe un entier $N$ tel que

$$
N^{*}\left[L_{s}^{\prime}\right]=0 \quad \text { dans } \operatorname{Pic}_{A / S}^{0}(k),
$$

où l'on a encore noté $N: \operatorname{Pic}_{A / S}^{0} \rightarrow \operatorname{Pic}_{A / S}^{0}$ le morphisme de la multiplication par $N$. Le groupe

$$
\operatorname{Ker}\left(\operatorname{Pic}_{A / S}^{0}\left(\mathfrak{o}_{n}\right) \rightarrow \operatorname{Pic}_{A / S}^{0}(k)\right)
$$


est de $p^{n}$-torsion en vertu de [44, 2.4]. On a donc

$$
\left(p^{n} N\right)^{*}\left[L_{n}^{\prime}\right]=0 \quad \text { dans } \operatorname{Pic}_{A / S}^{0}\left(\mathfrak{o}_{n}\right) .
$$

On désigne par $Y^{(n)}$ le $T$-schéma défini par le diagramme cartésien

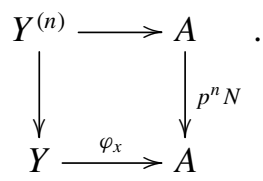

En vertu de (6.5.6), l'image réciproque de $L_{n}$ par $Y_{n}^{(n)} \rightarrow Y_{n}$ est triviale. D'après la théorie du corps de classes géométrique [42, VI 11], $Y_{\eta}^{(n)}$ est géométriquement connexe et est un revêtement étale et galoisien de $Y_{\eta}$. D'après (6.5.7), l'action du groupe $\operatorname{Aut}\left(Y_{\eta}^{(n)} / Y_{\eta}\right)$ s'étend en une $Y$-action sur $Y^{(n)}$. Le morphisme $Y^{(n)} \rightarrow Y$ est donc un $\eta$-revêtement fini et galoisien. La proposition résulte alors de 5.12(ii).

6.6. Soit $X$ une $\bar{S}$-courbe propre, $\bar{x}$ un $\bar{\eta}$-point de $X_{\bar{\eta}}$ et $n$ un entier $\geqslant 1$. On se propose de construire un foncteur de $\mathfrak{V}_{\check{X}}^{\text {DW }}$ dans $\operatorname{Rep}_{\mathfrak{o}_{n}}^{\text {lff }}\left(\pi_{1}\left(X_{\bar{\eta}}, \bar{x}\right)\right)$ (3.21). Soit $\mathcal{F}$ un fibré vectoriel de Deninger-Werner sur $\check{X}$. Comme $X$ est propre, le $\bar{\eta}$-point $\bar{x}$ se prolonge en une section $\bar{S} \rightarrow X$ que l'on note encore (abusivement) $\bar{x}$. On note $\bar{x}_{n}: \bar{S}_{n} \rightarrow X_{n}$ la réduction modulo $p^{n}$ de $\bar{x}$. On définit le $\mathfrak{o}_{n}$-module sous-jacent à la représentation associée à $\mathcal{F}$ par

$$
\mathbb{V}_{n}(\mathcal{F})=\Gamma\left(\bar{S}_{n}, \bar{x}_{n}^{*}\left(\mathcal{F}_{n}\right)\right),
$$

qui est un $\mathfrak{o}_{n}$-module libre de type fini.

D'après 5.12(i), il existe un $\bar{\eta}$-revêtement c.p.d.0 et galoisien $\varphi: X^{\prime} \rightarrow X$ de groupe des automorphismes $G$ tel que $\varphi_{n}^{*}\left(\mathcal{F}_{n}\right)$ soit libre de type fini. Soit $\left(C_{i}\right.$, $\left.\bar{y}_{i}\right)_{i \in I}$ un revêtement universel galoisien de $\left(X_{\bar{\eta}}, \bar{x}\right)$ (2.14). Choisissons un point géométrique $\bar{y} \in X_{\bar{\eta}}^{\prime}(\bar{\eta})$ au-dessus de $\bar{x}$. Il existe $i \in I$ et un épimorphisme

$$
\xi_{i}: C_{i} \rightarrow X_{\bar{\eta}}^{\prime} \quad \bar{y}_{i} \mapsto \bar{y}
$$

Celui-ci induit un épimorphisme

$$
\xi_{i}: \operatorname{Aut}\left(C_{i} / X_{\bar{\eta}}\right) \rightarrow G
$$

défini pour tout $\sigma \in \operatorname{Aut}\left(C_{i} / X_{\bar{\eta}}\right)$ par $\xi_{i}(\sigma)(\bar{y})=\xi_{i} \circ \sigma\left(\bar{y}_{i}\right)$. Le composé des homomorphismes

$$
\xi_{\bar{y}}: \pi_{1}\left(X_{\bar{\eta}}, \bar{x}\right) \rightarrow \operatorname{Aut}\left(C_{i} / X_{\bar{\eta}}\right)^{\mathrm{op}} \stackrel{\xi_{i}}{\rightarrow} G^{\mathrm{op}}
$$


est indépendant du choix de $i \in I$, mais dépend du choix de $\bar{y}$ au-dessus de $\bar{x}$. En effet, soit $\bar{y}$ et $\bar{y}^{\prime}$ deux $\bar{\eta}$-points de $X_{\bar{\eta}}^{\prime}$ au-dessus de $\bar{x}$. Il existe un élément $h \in G$ tel que $h(\bar{y})=\bar{y}^{\prime}$. D'après [12, Thm.13 (19)], les homomorphismes $\xi_{\bar{y}}, \xi_{\bar{y}^{\prime}}: \pi_{1}(C$, $\bar{x}) \rightarrow G^{\text {op }}$ sont reliés par:

$$
\xi_{\bar{y}^{\prime}}(\gamma)=h \xi_{\bar{y}}(\gamma) h^{-1}, \quad \forall \gamma \in \pi_{1}(C, \bar{x}) .
$$

6.7. Conservons les hypothèses et notations de 6.6. On construit une action $\rho_{n, \mathcal{F}}$ de $\pi_{1}\left(X_{\bar{\eta}}, \bar{x}\right)$ sur $\mathbb{V}_{n}(\mathcal{F})$. L'action de $G$ sur $X_{\bar{\eta}}^{\prime}$ s'étend en une $X$-action sur $X^{\prime}$. Tout $g \in G$ induit donc un automorphisme

$$
g_{n}^{*}: \Gamma\left(X_{n}^{\prime}, \varphi_{n}^{*}\left(\mathcal{F}_{n}\right)\right) \rightarrow \Gamma\left(X_{n}^{\prime}, \varphi_{n}^{*}\left(\mathcal{F}_{n}\right)\right) .
$$

Le $\bar{\eta}$-point $\bar{y}$ se prolonge en un $\bar{S}$-point de $X^{\prime}$ au-dessus de $\bar{x} \in X(\bar{S})$ que l'on note encore (abusivement) $\bar{y}$. Comme le morphisme structural $\lambda: X^{\prime} \rightarrow \bar{S}$ vérifie $\lambda_{*}\left(\mathscr{O}_{X^{\prime}}\right)=\mathscr{O}_{\bar{S}}$ universellement, le morphisme $\bar{y}_{n}: \bar{S}_{n} \rightarrow X_{n}^{\prime}$ déduit de $\bar{y}$ induit un isomorphisme :

$$
\bar{y}_{n}^{*}: \Gamma\left(X_{n}^{\prime}, \varphi_{n}^{*}\left(\mathcal{F}_{n}\right)\right) \stackrel{\sim}{\rightarrow} \Gamma\left(\bar{S}_{n}, \bar{y}_{n}^{*}\left(\varphi_{n}^{*}\left(\mathcal{F}_{n}\right)\right)\right)=\mathbb{V}_{n}(\mathcal{F}) .
$$

Pour tout $\gamma \in \pi_{1}\left(X_{\bar{\eta}}, \bar{x}\right)$, posant $g=\xi_{\bar{y}}(\gamma)$, on définit un automorphisme $\rho_{n, \mathcal{F}}(\gamma)$ de $\mathbb{V}_{n}(\mathcal{F})$ par

$$
\rho_{n, \mathcal{F}}(\gamma): \mathbb{V}_{n}(\mathcal{F}) \stackrel{\left(\bar{y}_{n}^{*}\right)^{-1}}{\rightarrow} \Gamma\left(X_{n}^{\prime}, \varphi_{n}^{*}\left(\mathcal{F}_{n}\right)\right) \stackrel{g_{n}^{*}}{\rightarrow} \Gamma\left(X_{n}^{\prime}, \varphi_{n}^{*}\left(\mathcal{F}_{n}\right)\right) \stackrel{\bar{y}_{n}^{*}}{\rightarrow} \mathbb{V}_{n}(\mathcal{F}) .
$$

On notera que $\rho_{n, \mathcal{F}}$ est l'homomorphisme composé

$$
\rho_{n, \mathcal{F}}: \pi_{1}\left(X_{\bar{\eta}}, \bar{x}\right) \stackrel{\xi_{\bar{Y}}}{\rightarrow} G^{\mathrm{op}} \rightarrow \operatorname{Aut}_{\mathfrak{o}_{n}} \Gamma\left(X_{n}^{\prime}, \varphi_{n}^{*}\left(\mathcal{F}_{n}\right)\right) \stackrel{\bar{y}_{n}^{*}}{\longrightarrow} \operatorname{Aut}_{\mathfrak{o}_{n}} \mathbb{V}_{n}(\mathcal{F}) .
$$

On définit la $\mathfrak{o}_{n}$-représentation de $\pi_{1}\left(X_{\bar{\eta}}, \bar{x}\right)$ associée à $\mathcal{F}$ par $\left(\mathbb{V}_{n}(\mathcal{F}), \rho_{n, \mathcal{F}}\right)$ que l'on note encore $\mathbb{V}_{n}(\mathcal{F})$.

Proposition 6.8 [12, Thm. 13]. Sous les hypothèses de 6.6, la $\mathfrak{o}_{n}$ représentation $\mathbb{V}_{n}(\mathcal{F})$ de $\pi_{1}\left(X_{\bar{\eta}}, \bar{x}\right)$ est indépendante des choix du $\bar{\eta}$-revêtement c.p.d.0 (5.4(ii)) et galoisien $\varphi: X^{\prime} \rightarrow X$ et du point $\bar{y}$ de $X_{\bar{\eta}}^{\prime}$ au-dessus de $\bar{x}$, à isomorphisme près.

6.9. Conservons les hypothèses et notations de 6.6. Étant donné un morphisme $f: \mathcal{F} \rightarrow \mathcal{F}^{\prime}$ de $\mathfrak{V}_{\check{X}}^{\text {DW }}$ et un entier $n \geqslant 1$, il existe un $\bar{\eta}$-revêtement c.p.d.0 et galoisien $\varphi: X^{\prime} \rightarrow X$ trivialisant $\mathcal{F}_{n}$ et $\mathcal{F}_{n}^{\prime}$ (cf. 5.12(i)). L'isomorphisme (6.7.2) est clairement fonctoriel en $\mathcal{F}$. D' après 6.8, le morphisme $f$ induit un morphisme de $\mathfrak{o}_{n}$-représentations de $\pi_{1}\left(X_{\bar{\eta}}, \bar{x}\right)$

$$
\mathbb{V}_{n}(f): \mathbb{V}_{n}(\mathcal{F}) \rightarrow \mathbb{V}_{n}\left(\mathcal{F}^{\prime}\right)
$$


La correspondance $\mathcal{F} \mapsto \mathbb{V}_{n}(\mathcal{F})$ définit ainsi un foncteur que l'on note (3.21)

$$
\mathbb{V}_{n}: \mathfrak{V}_{\check{X}}^{\mathrm{DW}} \rightarrow \operatorname{Rep}_{\mathfrak{o}_{n}}^{\mathrm{lff}}\left(\pi_{1}\left(X_{\bar{\eta}}, \bar{x}\right)\right)
$$

PROPOSITION 6.10 [12, Thm. 14 et 16]. (i) Les foncteurs $\left\{\mathbb{V}_{n}\right\}_{n \geqslant 1}$ (6.9.2) sont compatibles entre eux et définissent un foncteur o-linéaire et exact (3.21)

$$
\mathbb{V}: \mathfrak{V}_{\check{X}}^{\mathrm{DW}} \rightarrow \operatorname{Rep}_{\mathfrak{o}}^{\mathrm{ltf}}\left(\pi_{1}\left(X_{\bar{\eta}}, \bar{x}\right)\right),
$$

qui commute aux produits tensoriels, aux passages aux duaux et aux homomorphismes internes. On le notera aussi $\mathbb{V}_{\check{X}}$ pour signifier que la construction dépend de X.

(ii) Pour tout morphisme dominant $f: X^{\prime} \rightarrow X$ de $\bar{S}$-modèles de $X_{\bar{\eta}}$, l'image inverse d'un fibré vectoriel de Deninger-Werner sur $X$ par $f$ est de DeningerWerner sur $X^{\prime}$. De plus, on a un isomorphisme de foncteurs

$$
\mathbb{V}_{\check{X}} \stackrel{\sim}{\rightarrow} \mathbb{V}_{\check{X}^{\prime}} \circ f^{*}
$$

6.11. Dans la suite de cette section, on fixe une courbe propre et lisse $C$ sur $\bar{K}, \bar{x}$ un $\bar{\eta}$-point de $C$ et l'on pose $\check{C}=C \otimes_{\bar{K}} \mathfrak{C}$.

DÉFINITION 6.12. (i) On dit qu'un fibré vectoriel $F$ sur $\check{C}$ est de DeningerWerner s'il existe un $\bar{S}$-modèle $X$ de $C$ et un fibré vectoriel de DeningerWerner $\mathcal{F}$ sur $\check{X}$ muni d'un isomorphisme $F \simeq \mathcal{F}_{\bar{\eta}}$. On désigne par $\mathfrak{V}_{\check{C}}^{\mathrm{DW}}$ la sous-catégorie pleine de Vect $_{\check{C}}$ formée des fibrés vectoriels de DeningerWerner.

(ii) On dit qu'un fibré vectoriel $F$ sur $\check{C}$ est potentiellement de Deninger-Werner s'il existe un revêtement étale et connexe $\pi: C^{\prime} \rightarrow C$ tel que, posant $\check{\pi}=\pi \otimes_{\bar{K}} \mathfrak{C}, \check{\pi}^{*}(F)$ soit de Deninger-Werner sur $\check{C}^{\prime}=C^{\prime} \otimes_{\bar{K}} \mathfrak{C}$. On désigne par $\mathfrak{V}_{\check{C}}^{\mathrm{pDW}}$ la sous-catégorie pleine de Vect $_{\check{C}}$ formée des fibrés vectoriels potentiellement de Deninger-Werner.

THÉORÈME 6.13 [13, Thm. 11, 12,13]. (i) Les sous-catégories $\mathfrak{V}_{\check{C}}^{\mathrm{DW}}$ et $\mathfrak{V}_{\check{C}}^{\mathrm{pWW}}$ de $\operatorname{Vect}_{\check{C}}$ sont stables par extensions.

(ii) La catégorie $\mathfrak{V}_{\breve{C}}^{\mathrm{pDW}}$ contient tous les fibrés en droites de degré 0 sur $\check{C}$.

(iii) Tout fibré vectoriel de $\mathfrak{V}_{\breve{C}}^{\mathrm{pDW}}$ est semi-stable de degré 0 . 
6.14. Soit $X$ un $\bar{S}$-modèle de $C$. D'après 5.13, on a un foncteur pleinement fidèle $J_{\check{X}}: \mathfrak{V}_{\check{X}, \mathbb{Q}}^{\mathrm{DW}} \rightarrow \mathfrak{V}_{\check{C}}^{\mathrm{DW}}$. D'après $(3.21 .2),(6.10 .2)$ et $[12$, Cor. 21], on obtient un foncteur $\mathfrak{C}^{2}$-linéaire, exact (3.18) :

$$
\mathbb{V}_{\check{C}}: \mathfrak{V}_{\check{C}}^{\mathrm{DW}} \rightarrow \operatorname{Rep}_{\mathfrak{C}}^{\text {cont }}\left(\pi_{1}(C, \bar{x})\right)
$$

qui commute aux produits tensoriels, aux duaux et aux homomorphismes internes (cf. [13, Thm. 28]).

\section{Topos annelé de Faltings}

7.1. Dans cette section, on se donne un $S$-schéma plat, séparé et de type fini $X$ tel que $\bar{X}=X \times{ }_{S} \bar{S}$ (2.1.1) soit normal. On notera que $\bar{X}$ est localement irréductible dans le sens de [4, III.3.1]. En effet, comme $\bar{X}$ est $\bar{S}$-plat, ses points génériques sont les points génériques du schéma $X_{\bar{\eta}}$, qui est noethérien; l'ensemble des points génériques de $\bar{X}$ est donc fini (cf. [4, III.3.2(ii)]). On note $\hbar: \bar{X} \rightarrow X$ et $h: X_{\bar{\eta}} \rightarrow X$ les morphismes canoniques. On désigne par $E$ la catégorie des morphismes de schémas $V \rightarrow U$ au-dessus du morphisme canonique $X_{\bar{\eta}} \rightarrow X$, i.e. des diagrammes commutatifs

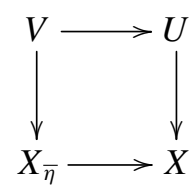

tels que le morphisme $U \rightarrow X$ soit étale et que le morphisme $V \rightarrow U_{\bar{\eta}}$ soit fini et étale [4, VI.10.1]. Elle est fibrée au-dessus de la catégorie $\mathbf{E}_{/ X}$ des $X$-schémas étales par le foncteur

$$
\pi: E \rightarrow \mathbf{E} \mathbf{t}_{/ X}, \quad(V \rightarrow U) \mapsto U .
$$

La fibre de $\pi$ au-dessus d'un $X$-schéma étale $U$ est la catégorie $\mathbf{E} \mathbf{t}_{\mathrm{f} / U_{\bar{\eta}}}$ des schémas finis étales au-dessus de $U_{\bar{\eta}}$, que l'on équipe de la topologie étale (2.13). La catégorie fibrée $\pi$ est alors un site fibré [6, VI 7.2.1].

On munit $E$ de la topologie co-évanescente [4, VI.5.3], c'est-à-dire de la topologie engendrée par les recouvrements $\left\{\left(V_{i} \rightarrow U_{i}\right) \rightarrow(V \rightarrow U)\right\}_{i \in I}$ des deux types suivants :

(v) $U_{i}=U$ pour tout $i \in I$ et $\left(V_{i} \rightarrow V\right)_{i \in I}$ est un recouvrement;

(c) $\left(U_{i} \rightarrow U\right)_{i \in I}$ est un recouvrement et $V_{i} \simeq U_{i} \times_{U} V$ pour tout $i \in I$.

On appelle topos de Faltings de $X$ et l'on note $\widetilde{E}$ le topos des faisceaux d'ensembles sur $E$. Si $F$ est un préfaisceau sur $E$, on note $F^{a}$ le faisceau associé. Signalons la description commode et simple suivante de $\widetilde{E}$. 
Proposition 7.2 [4, VI.5.10]. La donnée d'un faisceau $F$ sur E est équivalente à la donnée pour tout objet $U$ de $\mathbf{E ́}_{/ X}$ d'un faisceau $F_{U}$ de $U_{\bar{\eta} \text {,fét }}(2.13)$ et pour tout morphisme $f: U^{\prime} \rightarrow U$ de $\mathbf{E}^{\mathbf{t}}{ }_{X X}$ d'un morphisme $F_{U} \rightarrow\left(f_{\bar{\eta}}\right)_{\text {fét } *}\left(F_{U^{\prime}}\right)$, ces morphismes étant soumis à des relations de compatibilité, tels que, pour toute famille couvrante $\left(f_{n}: U_{n} \rightarrow U\right)_{n \in \Sigma}$ de $\mathbf{E}_{/ X}$, si pour tout $(m, n) \in \Sigma^{2}$, on pose $U_{m n}=U_{m} \times_{U} U_{n}$ et l'on note $f_{m n}: U_{m n} \rightarrow U$ le morphisme canonique, la suite de morphismes de faisceaux de $U_{\bar{\eta} \text {,fét }}$

$$
F_{U} \rightarrow \prod_{n \in \Sigma}\left(f_{n, \bar{\eta}}\right)_{\text {fét } *}\left(F_{U_{n}}\right) \rightrightarrows \prod_{n, m \in \Sigma}\left(f_{m n, \bar{\eta}}\right)_{\text {fét } *}\left(F_{U_{m n}}\right)
$$

soit exacte.

En vertu de la proposition précédente, on peut identifier tout faisceau $F$ sur

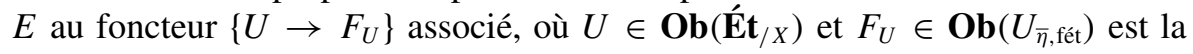
restriction de $F$ à la fibre de $\pi$ au-dessus de $U$.

7.3. Pour tout $U \in \mathbf{O b}\left(\mathbf{E} \mathbf{t}_{/ X}\right)$, on a un foncteur canonique :

$$
\alpha_{U !}: \mathbf{E}_{\mathbf{f} / U_{\bar{\eta}}} \rightarrow E, \quad V \mapsto(V \rightarrow U) .
$$

Le foncteur $\alpha_{X !}: \mathbf{E}_{\mathbf{f}_{\mathrm{f}} X_{\bar{\eta}}} \rightarrow E$ est continu et exact à gauche [4, VI.5.32]. Il définit donc un morphisme de topos [4, VI.(10.6.3)]

$$
\beta: \widetilde{E} \rightarrow X_{\bar{\eta}, \text { fét }} .
$$

Le foncteur

$$
\sigma^{+}: \mathbf{E} \mathbf{t}_{/ X} \rightarrow E, \quad U \mapsto\left(U_{\bar{\eta}} \rightarrow U\right)
$$

est continu et exact à gauche [4, VI.5.32]. Il définit donc un morphisme de topos [4, VI.(10.6.4)] :

$$
\sigma: \widetilde{E} \rightarrow X_{\text {ét }}
$$

Par ailleurs, le foncteur

$$
\Psi^{+}: E \rightarrow \mathbf{E}_{\mathbf{t}_{/ \bar{\eta}}}, \quad(V \rightarrow U) \mapsto V
$$

est continu et exact à gauche [4, VI.10.7]. Il définit donc un morphisme de topos :

$$
\Psi: X_{\bar{\eta}, \text { ét }} \rightarrow \widetilde{E} \text {. }
$$

On vérifie aussitôt que l'on a un isomorphisme canonique

$$
\beta \circ \Psi \stackrel{\sim}{\rightarrow} \rho_{X_{\bar{\eta}}}
$$




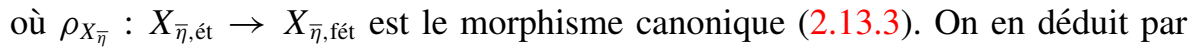
adjonction un morphisme

$$
\beta^{*} \rightarrow \Psi_{*} \rho_{X_{\bar{\eta}}}^{*},
$$

qui est un isomorphisme en vertu de [4, VI.10.9(iii)].

7.4. On désigne par $D$ la catégorie des morphismes de schémas $V \rightarrow U$ audessus du morphisme canonique $h: X_{\bar{\eta}} \rightarrow X$, i.e. des diagrammes commutatifs

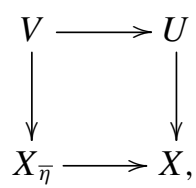

tels que les flèches verticales soient étales (cf. [4, VI.4.1]). Elle est fibrée audessus de la catégorie $\mathbf{E}_{/ X}$ des $X$-schémas étales, par le foncteur

$$
\varpi: D \rightarrow \mathbf{E}_{/ X} \quad(V \rightarrow U) \mapsto U .
$$

La fibre de $\varpi$ au-dessus d'un schéma étale $U$ est la catégorie $\mathbf{E ́ t}_{/ U_{\bar{\eta}}}$ des schémas étales au-dessus de $U_{\bar{\eta}}$, que l'on équipe de la topologie étale. La catégorie fibrée $\varpi$ est alors un site fibré (cf. [6, VI 7.2.1]).

On munit $D$ de la topologie co-évanescente [4, VI.5.3], c'est-à-dire la topologie engendrée par les recouvrements $\left\{\left(V_{i} \rightarrow U_{i}\right) \rightarrow(V \rightarrow U)\right\}_{i \in I}$ des deux types suivants :

(v) $U_{i}=U$ pour tout $i \in I$ et $\left(V_{i} \rightarrow V\right)_{i \in I}$ est un recouvrement;

(c) $\left(U_{i} \rightarrow U\right)_{i \in I}$ est un recouvrement et $V_{i} \simeq U_{i} \times_{U} V$ pour tout $i \in I$.

Le topos des faisceaux d'ensembles sur $D$ est appelé le topos co-évanescent et est noté $X_{\text {ét }} \overleftarrow{\times}_{X_{\text {ett }}} X_{\bar{\eta}, \text { ét. }}$. Celui-là vérifie une propriété universelle qui explique cette notation (cf. [4, VI.3.7 et VI.3.12]). Les foncteurs

$$
\begin{aligned}
& \mathrm{p}_{1}^{+}: \mathbf{E}^{\prime} \mathbf{t}_{/ X} \rightarrow D, \quad U \mapsto\left(U_{\bar{\eta}} \rightarrow U\right), \\
& \mathrm{p}_{2}^{+}: \mathbf{E}^{\prime} \mathbf{t}_{/ X_{\bar{\eta}}} \rightarrow D, \quad V \mapsto(V \rightarrow X),
\end{aligned}
$$

sont exacts à gauche et continus. Ils définissent donc deux morphismes de topos [6, IV 4.9.2]

$$
\begin{aligned}
& \mathrm{p}_{1}: X_{\text {ét }} \overleftarrow{\times}_{X_{\text {êt }}} X_{\bar{\eta}, \text { ét }} \rightarrow X_{\text {ét }}, \\
& \mathrm{p}_{2}: X_{\text {ét }} \overleftarrow{\times}_{X_{\text {êt }}} X_{\bar{\eta} \text {,ét }} \rightarrow X_{\bar{\eta} \text {,ét }}
\end{aligned}
$$


7.5. Tout objet de $E$ est naturellement un objet de $D$. On définit ainsi un $\left(\mathbf{E} \mathbf{t}_{/ X}\right)$ foncteur cartésien pleinement fidèle

$$
\rho^{+}: E \rightarrow D
$$

dont la fibre au-dessus d'un schéma étale $U$ sur $X$ est le foncteur d'injection canonique $\mathbf{E}_{\mathbf{f} / U_{\bar{\eta}}} \rightarrow \mathbf{E}^{\prime} \mathbf{t}_{/ U_{\bar{\eta}}}$. Le foncteur (7.5.1) est continu et exact à gauche [4, VI.10.15]. Il définit donc un morphisme de topos

$$
\rho: X_{\text {êt }} \overleftarrow{\times}_{X_{\text {et }}} X_{\bar{\eta}, \text { ét }} \rightarrow \widetilde{E}
$$

Il résulte aussitôt des définitions que les carrés du diagramme

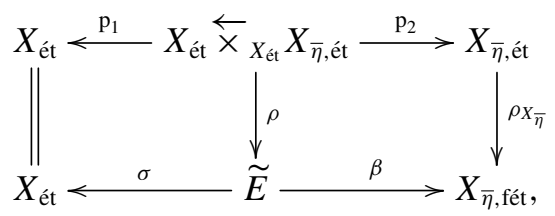

où $\rho_{X_{\bar{\eta}}}$ est le morphisme (2.13.3), sont commutatifs à isomorphismes canoniques près.

7.6. D'après [4, VI.3.11], la donnée d'un point de $X_{\text {ét }} \overleftarrow{x}_{X_{\text {êt }}} X_{\bar{\eta}, \text { ét }}$ est équivalente à la donnée d'une paire de points géométriques $\bar{x}$ de $X$ et $\bar{y}$ de $X_{\bar{\eta}}$ et d'une flèche de spécialisation $u: \bar{y} \rightarrow X_{(\bar{x})}$, où $X_{(\bar{x})}$ est le localisé strict de $X$ en $\bar{x}$. Un tel point sera noté $(\bar{y} \rightsquigarrow \bar{x})$. On désigne par $\rho(\bar{y} \rightsquigarrow \bar{x})$ son image par $\rho$, qui est donc un point de $\widetilde{E}$. D'après [4, VI.5.30 et VI.10.18], lorsque $(\bar{y} \rightsquigarrow \bar{x})$ décrit la famille des points de $X_{\text {et }} \overleftarrow{\times}_{X_{\text {et }}} X_{\bar{\eta} \text {,ét }}$, la famille des foncteurs fibres de $X_{\text {ét }} \overleftarrow{\times}_{X_{\text {et }}} X_{\bar{\eta} \text {,ét }}$ (resp. $\widetilde{E})$ associés aux points $(\bar{y} \rightsquigarrow \bar{x})($ resp. $\rho(\bar{y} \rightsquigarrow \bar{x})$ ) est conservative.

7.7. Reprenons les notations de 2.13. On désigne par

$$
\varpi_{\mathrm{scoh}}: D_{\mathrm{scoh}} \rightarrow \mathbf{E}_{\mathrm{scoh} / X}, \quad \pi_{\mathrm{scoh}}: E_{\mathrm{scoh}} \rightarrow \dot{\mathbf{E}} \mathbf{t}_{\mathrm{scoh} / X}
$$

les sites fibrés déduits de $\varpi$ (7.4.2) et $\pi$ (7.1.2) par changement de base par le foncteur d'injection canonique

$$
\mathbf{E}_{\mathbf{s c o h}_{X}} \rightarrow \mathbf{E}_{\mathbf{t}} / X
$$

et par $\iota_{1}: D_{\text {scoh }} \rightarrow D$ et $\iota_{2}: E_{\text {scoh }} \rightarrow E$ les projections canoniques. D'après [4, VI.5.21], si l'on munit $D_{\text {scoh }}$ (resp. $E_{\text {scoh}}$ ) de la topologie co-évanescente définie par $\varpi_{\text {scoh }}\left(\right.$ resp. $\left.\pi_{\text {scoh }}\right)$ et si l'on note $\widetilde{D}_{\text {scoh }}\left(\right.$ resp. $\left.\widetilde{E}_{\text {scoh }}\right)$ le topos des faisceaux d'ensembles sur $D_{\text {scoh }}$ (resp. $E_{\text {scoh }}$ ), le foncteur $\iota_{1}$ (resp. $\iota_{2}$ ) induit par restriction une équivalence de catégories $X_{\text {ét }} \overleftarrow{\times}_{X_{\text {et }}} X_{\bar{\eta} \text {,ét }} \stackrel{\sim}{\rightarrow} \widetilde{D}_{\text {scoh }}$ (resp. $\widetilde{E} \stackrel{\sim}{\rightarrow} \widetilde{E}_{\text {scoh }}$ ). 
7.8. Pour tout $(V \rightarrow U) \in \mathbf{O b}(E)$, on note $\bar{U}^{V}$ la clôture intégrale de $\bar{U}$ dans $V$. On désigne par $\overline{\mathscr{B}}$ le préfaisceau d'anneaux sur $E$ défini pour tout $(V \rightarrow U) \in$ $\mathbf{O b}(E)$ par

$$
\overline{\mathscr{B}}(V \rightarrow U)=\Gamma\left(\bar{U}^{V}, \mathscr{O}_{\bar{U}^{V}}\right) .
$$

Pour tout $U \in \mathbf{O b}\left(\mathbf{E ́}_{/ X}\right)$, on pose (7.3.1)

$$
\overline{\mathscr{B}}_{U}=\overline{\mathscr{B}} \circ \alpha_{U !}
$$

D'après [4, III.8.16], $\overline{\mathscr{B}}$ est un faisceau pour la topologie co-évanescente de $E$. On dira dans la suite que $\overline{\mathscr{B}}$ est l'anneau de $\widetilde{E}$ associé à $\bar{X}$. Pour tout entier $n \geqslant 1$ et tout $U \in \mathbf{O b}\left(\mathbf{E} \mathbf{t}_{/ X}\right)$, on pose

$$
\begin{aligned}
\overline{\mathscr{B}}_{n} & =\overline{\mathscr{B}} / p^{n} \overline{\mathscr{B}} \\
\overline{\mathscr{B}}_{U, n} & =\overline{\mathscr{B}}_{U} / p^{n} \overline{\mathscr{B}}_{U} .
\end{aligned}
$$

La correspondance $\left\{U \mapsto \overline{\mathscr{B}}_{U, n}\right\}$ forme naturellement un préfaisceau sur $E$ dont le faisceau associé est canoniquement isomorphe à $\overline{\mathscr{B}}_{n}$ (cf. [4, VI.8.2 et VI.8.9]).

7.9. Considérons l'homomorphisme

$$
\hbar_{*}\left(\mathscr{O}_{\bar{X}}\right) \rightarrow \sigma_{*}(\overline{\mathscr{B}}),
$$

où $\hbar: \bar{X} \rightarrow X$ est la projection canonique, défini pour tout $U \in \mathbf{O b}\left(\mathbf{E ́}_{/ X}\right)$ par l'homomorphisme canonique

$$
\Gamma\left(\bar{U}, \mathscr{O}_{\bar{U}}\right) \rightarrow \Gamma\left(\bar{U}^{U_{\bar{\eta}}}, \mathscr{O}_{\bar{U}} U_{\bar{\eta}}\right)
$$

Sauf mention explicite du contraire, on considère $\sigma: \widetilde{E} \rightarrow X_{\text {ét }}(7.3 .4)$ comme un morphisme de topos annelés, respectivement par $\overline{\mathscr{B}}$ et $\hbar_{*}\left(\mathscr{O}_{\bar{X}}\right)$. Nous utilisons pour les modules la notation $\sigma^{-1}$ pour désigner l'image inverse au sens des faisceaux abéliens et nous réservons la notation $\sigma^{*}$ pour l'image inverse au sens des modules.

7.10. Comme $X_{\eta}$ est un ouvert de $X_{\text {ét }}$, i.e. un sous-objet de l'objet final $X$ de $\mathbf{E ́ t}_{/ X}$ [6, IV 8.3], $\sigma^{*}\left(X_{\eta}\right)$ est un ouvert de $\widetilde{E}$. On note $\gamma: \widetilde{E}_{/ \sigma^{*}\left(X_{\eta}\right)} \rightarrow \widetilde{E}$ le morphisme de localisation de $\widetilde{E}$ en $\sigma^{*}\left(X_{\eta}\right)$. On désigne par $\widetilde{E}_{s}$ le sous-topos fermé de $\widetilde{E}$ complémentaire de l'ouvert $\sigma^{*}\left(X_{\eta}\right)$, i.e. la sous-catégorie pleine de $\widetilde{E}$ formée des faisceaux $F$ tels que $\gamma^{*}(F)$ soit un objet final de $\widetilde{E}_{/ \sigma^{*}\left(X_{\eta}\right)}$ [6, IV 9.3.5]. On note

$$
\delta: \widetilde{E}_{s} \rightarrow \widetilde{E}
$$


le plongement canonique, c'est-à-dire le morphisme de topos tel que $\delta_{*}: \widetilde{E}_{s} \rightarrow \widetilde{E}$ soit le foncteur d'injection canonique. D'après [4, III.9.7], pour tout entier $n \geqslant 1$, l'anneau $\overline{\mathscr{B}}_{n}$ (7.8.3) est un objet de $\widetilde{E}_{s}$.

7.11. Soit $n$ un entier $\geqslant 1$. Notons $d: X_{s} \rightarrow X$ et $d_{n}: X_{s} \rightarrow X_{n}$ les morphismes canoniques (2.1.1). Il existe un morphisme de topos [4, III.9.8] :

$$
\sigma_{s}: \widetilde{E}_{s} \rightarrow X_{s, \text { ét }}
$$

unique à isomorphisme près tel que le diagramme

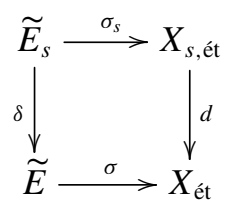

soit commutatif à isomorphisme près.

Pour tout entier $n \geqslant 1$, comme le corps résiduel de $\mathcal{O}_{\bar{K}}$ est algébriquement clos, on a une immersion fermée $\bar{d}_{n}: X_{s} \rightarrow \bar{X}_{n}$ qui relève $d_{n}$. Comme $\bar{d}_{n}$ est un homéomorphisme universel, on peut considérer le faisceau $\mathscr{O}_{\bar{X}_{n}}$ de $\bar{X}_{n \text {,ét }}$ (ou de $\bar{X}_{n, \text { zar }}$ ) comme un faisceau de $X_{s, \text { ét }}$ (ou de $X_{s, \text { zar }}$ ). L'homomorphisme canonique $\sigma^{-1}\left(\hbar_{*}\left(\mathscr{O}_{\bar{X}}\right)\right) \rightarrow \overline{\mathscr{B}}$ (7.9.1) induit alors un homomorphisme d'anneaux sur $\widetilde{E}_{s}$ [4, III.9.9]:

$$
\sigma_{s}^{-1}\left(\mathscr{O}_{\bar{X}_{n}}\right) \rightarrow \overline{\mathscr{B}}_{n}
$$

Par suite, le morphisme de topos $\sigma_{s}$ (7.11.1) est sous-jacent à un morphisme de topos annelés que l'on note

$$
\sigma_{n}:\left(\widetilde{E}_{s}, \overline{\mathscr{B}}_{n}\right) \rightarrow\left(X_{s, \text { ét }}, \mathscr{O}_{\bar{X}_{n}}\right) .
$$

7.12. On désigne par $\breve{\breve{B}}$ l'anneau $\left(\overline{\mathscr{B}}_{n}\right)_{n \geqslant 1}$ de $\widetilde{E}_{s}^{\mathbb{N}^{\circ}}(2.11)$ et par $\mathscr{O}_{\overline{\bar{X}}}$ l'anneau $\left(\mathscr{O}_{\bar{X}_{n}}\right)_{n \geqslant 1}$ de $X_{s, \text { ét }}^{\mathbb{N}^{\circ}}\left(\right.$ ou de $X_{s, \text { zar }}^{\mathbb{N}^{\circ}}$ ). Les morphismes $\left(\sigma_{n}\right)_{n \geqslant 1}$ (7.11.4) induisent un morphisme de topos annelés:

$$
\breve{\sigma}:\left(\widetilde{E}_{s}^{\mathbb{N}^{\circ}}, \breve{\mathscr{B}}\right) \rightarrow\left(X_{s, \text { ét }}^{\mathbb{N}^{\circ}}, \mathscr{O}_{\breve{X}}\right) .
$$

On note $\mathfrak{X}$ le schéma formel complété $p$-adique de $\bar{X}$. On désigne par:

$$
\breve{u}:\left(X_{s, \text { ét }}^{\mathbb{N}^{\circ}}, \mathscr{O}_{\breve{X}}\right) \rightarrow\left(X_{s, \text { zar }}^{\mathbb{N}^{\circ}}, \mathscr{O}_{\bar{X}}\right)
$$

le morphisme canonique, par

$$
\lambda:\left(X_{s, \mathrm{zar}}^{\mathbb{N}^{\circ}}, \mathscr{O}_{\overline{\bar{X}}}\right) \rightarrow\left(X_{s, \mathrm{zar}}, \mathscr{O}_{\mathfrak{X}}\right)
$$


le morphisme de topos annelés dont le foncteur image directe est la limite projective (2.11) et par

$$
\mathrm{T}:\left(\widetilde{E}_{s}^{\mathbb{N}^{\circ}}, \breve{\mathscr{B}}\right) \rightarrow\left(X_{s, \mathrm{zar}}, \mathscr{O}_{\mathfrak{X}}\right)
$$

le morphisme composé de topos annelés $\lambda \circ \breve{u} \circ \breve{\sigma}$.

7.13. Si $A$ est un anneau, on note encore $A$ le faisceau constant de valeur $A$ de $X_{\bar{\eta} \text {,fét. }}$ Comme l'anneau $\overline{\mathscr{B}}\left(X_{\bar{\eta}} \rightarrow X\right)=\Gamma\left(\bar{X}, \mathscr{O}_{\bar{X}}\right)$ est une $\mathcal{O}_{\bar{K}}$-algèbre, il existe

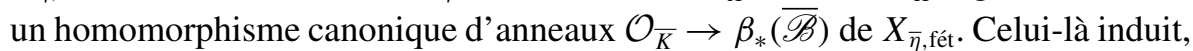
pour tout entier $n \geqslant 1$, un homomorphisme canonique $\mathfrak{o}_{n} \rightarrow \beta_{*}\left(\overline{\mathscr{B}}_{n}\right)$ de $X_{\bar{\eta} \text {,fét. On }}$. définit le morphisme de topos annelés

$$
\beta_{n}:\left(\widetilde{E}_{s}, \overline{\mathscr{B}}_{n}\right) \rightarrow\left(X_{\bar{\eta}, \text { fét }}, \mathfrak{o}_{n}\right)
$$

par le morphisme de topos composé $\beta \circ \delta(7.3 .2,7.10 .1)$ et l'homomorphisme canonique $\mathfrak{o}_{n} \rightarrow \beta_{*}\left(\overline{\mathscr{B}}_{n}\right)$. On note $\breve{o}$ l'anneau $\left(\mathfrak{o}_{n}\right)_{n \geqslant 1}$ de $X_{\bar{\eta}, \text { fét }}^{\mathbb{N}^{\circ}}$ et

$$
\breve{\beta}:\left(\widetilde{E}_{s}^{\mathbb{N}^{\circ}}, \breve{\mathscr{B}}\right) \rightarrow\left(X_{\bar{\eta}, \text { fét }}^{\mathbb{N}^{\circ}}, \breve{\mathfrak{o}}\right)
$$

le morphisme de topos annelés induit par les morphismes $\left(\beta_{n}\right)_{n \geqslant 1}(7.13 .1)$.

7.14. Pour qu'un faisceau $F$ de $\widetilde{E}$ soit un objet de $\widetilde{E}_{s}$, il faut et il suffit que, pour tout point $(\bar{y} \rightsquigarrow \bar{x})$ de $X_{\text {ét }} \overleftarrow{x}_{X_{\text {êt }}} X_{\bar{\eta}, \text { ét }}(7.5)$ tel que $\bar{x}$ soit au-dessus de $\eta$, la fibre $F_{\rho(\bar{y} \rightsquigarrow \bar{x})}$ de $F$ en $\rho(\bar{y} \rightsquigarrow \bar{x})$ soit un singleton (cf. [4, III.9.6]). Soit $n$ un entier $\geqslant 1$ et $\mathbb{L}$ un $\mathfrak{o}_{n}$-module de $X_{\bar{\eta} \text {,fét. }}$. Comme les foncteurs fibres commutent au produit tensoriel [6, IV 13.4] et que $\overline{\mathscr{B}}_{n}$ est un objet de $\widetilde{E}_{s}$ [4, III.9.7], on en déduit que

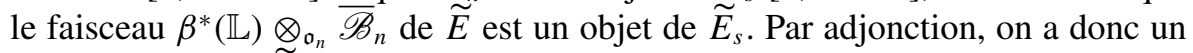
isomorphisme de $\widetilde{E}$

$$
\beta^{*}(\mathbb{L}) \otimes_{\mathfrak{o}_{n}} \overline{\mathscr{B}}_{n} \stackrel{\sim}{\rightarrow} \delta_{*}\left(\beta_{n}^{*}(\mathbb{L})\right) .
$$

7.15. On note $\operatorname{Mod}\left(\overline{\mathscr{B}}_{n}\right)$ la catégorie des $\overline{\mathscr{B}}_{n}$-modules de $\widetilde{E}_{s}$ et $\operatorname{Mod}^{\mathrm{tf}}\left(\overline{\mathscr{B}}_{n}\right)$ la sous-catégorie pleine formée des $\overline{\mathscr{B}}_{n}$-modules de type fini. On note $\operatorname{Mod}(\breve{\mathscr{B}})$ la catégorie des $\breve{\mathscr{B}}$-modules de $\widetilde{E}_{s}^{\mathbb{N}^{\circ}}, \operatorname{Mod}^{\text {atf }}(\stackrel{\breve{\mathscr{B}}}{)})$ la sous-catégorie pleine formée des $\breve{\mathscr{B}}$-modules adiques de type fini $(2.11)$ et $\operatorname{Mod}_{\mathbb{Q}}(\breve{\mathscr{B}})\left(\operatorname{resp} . \operatorname{Mod}_{\mathbb{Q}}^{\text {atf }}(\breve{\mathscr{B}})\right)$ la catégorie des objets de $\operatorname{Mod}\left(\frac{\breve{\mathscr{B}}}{)}\left(\operatorname{resp} \cdot \operatorname{Mod}^{\text {atf }}\left(\frac{\breve{\mathscr{B}}}{)}\right)\right.\right.$ à isogénie près (2.16).

7.16. Supposons, dans la suite de cette section, que $X$ soit un $S$-schéma propre à réduction semi-stable de fibre géométrique générique connexe (5.9). On notera 
que $X$ est adéquat dans le sens de [4, III.4.7] et par suite que $\bar{X}$ est normal [4, III.4.2 (iii)]. D'après $[5,6.1]$, pour tout point géométrique $\bar{x}$ de $X$, il existe un voisinage étale $U$ de $\bar{x}$ dans $X$ dont la fibre générique $U_{\eta}$ est un schéma $K(\pi$, 1) (cf. $[3,1.2 .2])$. On en déduit, pour tout faisceau abélien de torsion localement constant et constructible $\mathscr{F}$ de $X_{\bar{\eta} \text {,ét }}$ et tout entier $i \geqslant 1$, que $\mathrm{R}^{i} \Psi_{*}(\mathscr{F})=0$ (7.3.6) (cf. $[5,9.6])$. On a donc un isomorphisme

$$
\mathrm{H}^{i}\left(\widetilde{E}, \Psi_{*}(\mathscr{F})\right) \stackrel{\sim}{\rightarrow} \mathrm{H}^{i}\left(X_{\bar{\eta}, \text { ét }}, \mathscr{F}\right)
$$

déduit de la suite spectrale de Cartan-Leray.

7.17. Soit $\mathbb{L}$ un faisceau abélien de torsion de $X_{\bar{\eta} \text {,fét }}$. Le faisceau $\rho_{X_{\bar{\eta}}}^{*}(\mathbb{L})(2.13 .3)$ est isomorphe à une limite inductive de faisceaux abéliens de torsion localement constants et constructibles de $X_{\bar{\eta} \text {,ét }}$ (cf. [4, VI.9.20]). D'après [4, VI.10.5 et VI.10.10], les topos $X_{\bar{\eta}, \text { ét }}$ et $\widetilde{E}$ et le morphisme de topos $\Psi$ sont cohérents. Par (7.16.1) et passage à la limite [6, VI 5.1], on a un isomorphisme

$$
\mathrm{H}^{i}\left(\widetilde{E}, \Psi_{*}\left(\rho_{X_{\bar{\eta}}}^{*}(\mathbb{L})\right)\right) \stackrel{\sim}{\rightarrow} \mathrm{H}^{i}\left(X_{\bar{\eta}, \text { ét }}, \rho_{X_{\bar{\eta}}}^{*}(\mathbb{L})\right)
$$

déduit de la suite spectrale de Cartan-Leray. Pour tout entier $i \geqslant 0$, considérons le morphisme composé

$$
\begin{aligned}
& \mathrm{H}^{i}\left(X_{\bar{\eta}, \text { fét }}, \mathbb{L}\right) \stackrel{\beta^{*}}{\rightarrow} \mathrm{H}^{i}\left(\widetilde{E}, \beta^{*}(\mathbb{L})\right) \\
& \quad \stackrel{\sim}{\rightarrow} \mathrm{H}^{i}\left(\widetilde{E}, \Psi_{*}\left(\rho_{X_{\bar{\eta}}}^{*}(\mathbb{L})\right)\right) \stackrel{\sim}{\rightarrow} \mathrm{H}^{i}\left(X_{\bar{\eta}, \text { ét }}, \rho_{X_{\bar{\eta}}}^{*}(\mathbb{L})\right),
\end{aligned}
$$

où la deuxième flèche est induite par l'isomorphisme $\beta^{*} \stackrel{\sim}{\rightarrow} \Psi_{*} \rho_{\bar{X}_{\bar{\eta}}}^{*}$ (7.3.8) et la troisième flèche est l'isomorphisme (7.17.1). D'après 2.12, le morphisme composé (7.17.3) s'identifie au morphisme induit par $\rho_{X_{\bar{\eta}}}^{*}$ :

$$
\rho_{X_{\bar{\eta}}}^{*}: \mathrm{H}^{i}\left(X_{\bar{\eta}, \text { fét }}, \mathbb{L}\right) \rightarrow \mathrm{H}^{i}\left(X_{\bar{\eta}, \text { ét }}, \rho_{X_{\bar{\eta}}}^{*}(\mathbb{L})\right) .
$$

qui est un isomorphisme pour $i=0,1$ et un monomorphisme pour $i=2$ (cf. 2.15). Comme les deux dernières flèches de (7.17.2) sont des isomorphismes, on en déduit le résultat suivant.

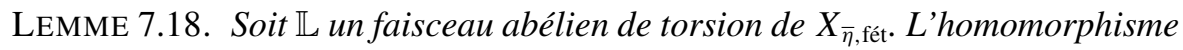

$$
\beta^{*}: \mathrm{H}^{i}\left(X_{\bar{\eta}, \text { fét }}, \mathbb{L}\right) \rightarrow \mathrm{H}^{i}\left(\widetilde{E}, \beta^{*}(\mathbb{L})\right)
$$

est un isomorphisme si $i=0,1$, et est un monomorphisme si $i=2$. 
THÉORÈME 7.19 (Faltings; $[3,2.4 .16]$ ). Soit $n$ un entier $\geqslant 1$ et $\mathbb{L}$ un $\mathfrak{o}_{n}$-module localement libre de type fini de $X_{\bar{\eta}}$,ét. Le morphisme $\mathfrak{o}_{n}$-linéaire canonique

$$
\mathrm{H}^{i}\left(\widetilde{E}, \beta^{*}(\mathbb{L})\right) \rightarrow \mathrm{H}^{i}\left(\widetilde{E}, \beta^{*}(\mathbb{L}) \otimes_{\mathfrak{o}_{n}} \overline{\mathscr{B}}_{n}\right)
$$

est un $\alpha$-isomorphisme (3.1).

Preuve. D'après [4, VI.9.20], il existe une extension finie $F$ de $\mathbb{Q}_{p}$ contenue dans $\bar{K}$ et, notant $\mathcal{O}_{F}$ l'anneau de valuation de $F$, un $\left(\mathcal{O}_{F} / p^{n} \mathcal{O}_{F}\right)$-module localement libre de type fini $\mathbb{L}^{\prime}$ de $X_{\bar{\eta} \text {,fét }}$ tels que $\mathbb{L} \simeq \mathbb{L}^{\prime} \otimes_{\mathcal{O}_{F} / p^{n} \mathcal{O}_{F}} \mathfrak{o}_{n}$. Comme $\mathfrak{o}_{n}$ est plat sur $\mathcal{O}_{F} / p^{n} \mathcal{O}_{F}$, il suffit de démontrer que le morphisme $\mathfrak{o}_{n}$-linéaire canonique

$$
\mathrm{H}^{i}\left(\widetilde{E}, \beta^{*}\left(\mathbb{L}^{\prime}\right)\right) \otimes_{\mathcal{O}_{F} / p^{n} \mathcal{O}_{F}} \mathfrak{o}_{n} \rightarrow \mathrm{H}^{i}\left(\widetilde{E}, \beta^{*}\left(\mathbb{L}^{\prime}\right) \otimes_{\mathcal{O}_{F} / p^{n} \mathcal{O}_{F}} \overline{\mathscr{B}}_{n}\right)
$$

est un $\alpha$-isomorphisme. La même démonstration que celle de [3, 2.4.16] s'applique alors aux $\left(\mathcal{O}_{F} / p^{n} \mathcal{O}_{F}\right)$-modules localement libres de type fini de $X_{\bar{\eta} \text {,fét, }}$, en remplaçant le morphisme de Frobenius par une puissance de ce dernier.

Proposition 7.20. Soit $n$ un entier $\geqslant 1$ et $\mathbb{L}$ un $\mathfrak{o}_{n}$-module localement libre de

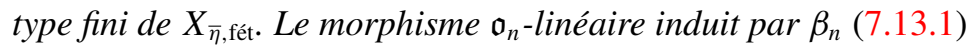

$$
\beta_{n}^{*}: \mathrm{H}^{i}\left(X_{\bar{\eta}, \text { fét }}, \mathbb{L}\right) \rightarrow \mathrm{H}^{i}\left(\widetilde{E}_{s}, \beta_{n}^{*}(\mathbb{L})\right)
$$

est un $\alpha$-isomorphisme si $i=0,1$ et un $\alpha$-monomorphisme si $i=2$ (3.1).

Preuve. Comme le foncteur $\delta_{*}: \widetilde{E}_{s} \rightarrow \widetilde{E}$ (7.10.1) est exact, on a un isomorphisme

$$
\delta^{*}: \mathrm{H}^{i}\left(\widetilde{E}, \beta^{*}\left(\mathbb{L}_{n}\right) \otimes_{\mathfrak{o}_{n}} \overline{\mathscr{B}}_{n}\right) \stackrel{\sim}{\rightarrow} \mathrm{H}^{i}\left(\widetilde{E}_{s}, \beta_{n}^{*}\left(\mathbb{L}_{n}\right)\right)
$$

en vertu de (7.14.1). Le morphisme (7.20.1) s'identifie au morphisme composé

$$
\mathrm{H}^{i}\left(X_{\bar{\eta}, \text { fét }}, \mathbb{L}\right) \stackrel{\beta^{*}}{\rightarrow} \mathrm{H}^{i}\left(\widetilde{E}, \beta^{*}(\mathbb{L})\right) \rightarrow \mathrm{H}^{i}\left(\widetilde{E}, \beta^{*}(\mathbb{L}) \bigotimes_{\mathfrak{o}_{n}} \overline{\mathscr{B}}_{n}\right) \stackrel{\delta^{*}}{\rightarrow} \mathrm{H}^{i}\left(\widetilde{E}_{s}, \beta_{n}^{*}\left(\mathbb{L}_{n}\right)\right),
$$

où la première flèche est le morphisme (7.18.1) et la deuxième flèche est le $\alpha$ isomorphisme (7.19.1). La proposition s'ensuit compte tenu de 7.18 et 7.19.

Corollaire 7.21. Soit $\mathbb{L}=\left(\mathbb{L}_{n}\right)_{n \geqslant 1}$ un $\breve{\mathfrak{o}}$-module localement libre de type fini de $X_{\bar{\eta} \text {,fét. }}^{\mathbb{N}^{\circ}}$ Pour $i=0,1$, le morphisme o-linéaire induit par $\breve{\beta}$ (7.13.2)

$$
\breve{\beta}^{*}: \mathrm{H}^{i}\left(X_{\bar{\eta}, \text { fét }}^{\mathbb{N}^{\circ}}, \mathbb{L}\right) \rightarrow \mathrm{H}^{i}\left(\widetilde{E}_{s}^{\mathbb{N}^{\circ}}, \breve{\beta}^{*}(\mathbb{L})\right)
$$

est un $\alpha$-isomorphisme. 
Preuve. Pour tout $i \geqslant 0$, on a un diagramme commutatif (cf. [4, VI.7.10])

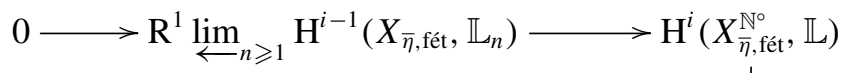

$$
\begin{aligned}
& \left(\beta_{n}^{*}\right) \downarrow \\
& 0 \longrightarrow \mathrm{R}^{1}{\underset{\lim }{\longleftarrow}}_{n \geqslant 1} \mathrm{H}^{i-1}\left(\widetilde{E}_{s}, \beta_{n}^{*}\left(\mathbb{L}_{n}\right)\right) \longrightarrow \mathrm{H}^{i}\left(\widetilde{E}_{s}^{\mathbb{N}^{0}}, \breve{\beta}^{*}(\mathbb{L})\right) \\
& \mathrm{H}^{i}\left(X_{\bar{\eta}, \text { fét }}^{\mathbb{N}^{\circ}}, \mathbb{L}\right) \longrightarrow \lim _{n \geqslant 1} \mathrm{H}^{i}\left(X_{\bar{\eta}, \text { fét }}, \mathbb{L}_{n}\right) \longrightarrow 0 \\
& \breve{\beta}^{*} \downarrow \\
& \mathrm{H}^{i}\left(\widetilde{E}_{s}^{\mathbb{N}^{\circ}}, \breve{\beta}^{*}(\mathbb{L})\right) \longrightarrow \lim _{n \geqslant 1} \mathrm{H}^{i}\left(\widetilde{E}_{s}, \beta_{n}^{*}\left(\mathbb{L}_{n}\right)\right) \longrightarrow 0
\end{aligned}
$$

où 1 'on a posé $\mathrm{H}^{-1}\left(X_{\bar{\eta}, \text { fét }}, \mathbb{L}_{n}\right)=0$ et $\mathrm{H}^{-1}\left(\widetilde{E}_{s}, \beta_{n}^{*}\left(\mathbb{L}_{n}\right)\right)=0$ pour tout $n \geqslant 1$. L'assertion résulte alors de 3.10(ii) et 7.20.

7.22. On désigne par $\widetilde{E}_{p t}$ le topos de Faltings associé au morphisme canonique $h_{S}: \bar{\eta} \rightarrow S$. Pour tout $U \in \mathbf{O b}\left(\mathbf{E}_{\mathbf{t}} / S\right)$, le site $\mathbf{E}_{\mathbf{f}} / U_{\bar{\eta}}$ est équivalent au site $\mathbf{E} \mathbf{t}_{\mathrm{coh} / U_{\bar{\pi}}}$ (2.13). D'après [4, VI.5.11], le foncteur $\rho^{+}$induit une équivalence de topos (7.5.2):

$$
\rho: S_{\text {ét }} \overleftarrow{\times}_{S_{\text {et }}} \bar{\eta}_{\text {ét }} \stackrel{\sim}{\rightarrow} \widetilde{E}_{p t}
$$

qui s'insère dans le diagramme commutatif (7.5.3)

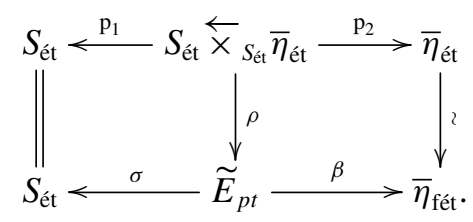

7.23. Soit $a: \bar{S} \rightarrow S, h_{S}: \bar{\eta} \rightarrow S$ les morphismes canoniques, et $a^{+}:$ $\mathbf{E}_{\text {scoh } / S} \rightarrow \mathbf{E}^{\prime} \mathbf{t}_{\text {scoh } / S}$ et $h_{S}^{+}: \mathbf{E ́}_{\text {scoh } / S} \rightarrow \mathbf{E}_{\text {coh } / \bar{\eta}}$ les foncteurs de changement de base associés (2.13). On note $D_{\text {scoh }}$ et $E_{\text {scoh }}$ les sites introduits dans 7.7 relativement au morphisme $h_{S}$. On observera que $\bar{\eta}_{\text {ét }}$ étant un topos ponctuel, il existe un unique morphisme de topos

$$
b: \bar{S}_{\text {ét }} \rightarrow \bar{\eta}_{\text {ét }},
$$

tel que $b^{*}(\bar{\eta})=\bar{S}[6$, IV 4.3]. Il est induit par l'unique foncteur exact à gauche

$$
b^{+}: \mathbf{E}_{\mathbf{c}_{\mathrm{coh} / \bar{\eta}}} \rightarrow \mathbf{E}_{\mathbf{t}} \mathbf{s c o h} / \bar{S}
$$




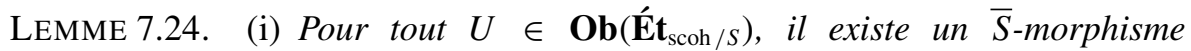
fonctoriel en $U$

$$
\bar{U} \rightarrow b^{+}\left(U_{\bar{\eta}}\right)
$$

induisant un isomorphisme au-dessus de $\bar{\eta}$.

(ii) Pour tout $(V \rightarrow U) \in \mathbf{O b}\left(E_{\mathrm{scoh}}\right)$, il existe un isomorphisme de $\bar{S}$-schémas fonctoriel en $(V \rightarrow U)$

$$
\bar{U} \times_{b^{+}\left(U_{\bar{\eta}}\right)} b^{+}(V) \stackrel{\sim}{\rightarrow} \bar{U}^{V},
$$

où $\bar{U}^{V}$ désigne la clôture intégrale de $\bar{U}$ dans $V$ (7.8).

Preuve. D'après [25, 18.10.8], tout objet $U$ de $\mathbf{E}_{\text {scoh } / S}$ est la somme de deux sous-schémas ouverts $U_{1}$ et $U_{2}$, où $U_{1}$ est fini sur $S$ et $U_{2}$ est fini sur $\eta$. On peut traiter indépendamment le cas où $U$ est fini sur $S$ et celui où $U$ est fini sur $\eta$.

Si $U$ est fini sur $S, U$ est isomorphe à une somme de copies de $S$. Par suite, on a un isomorphisme canonique $\bar{U} \stackrel{\sim}{\rightarrow} b^{+}\left(\bar{U}_{\bar{\eta}}\right)$, d'où le lemme dans ce cas.

Si $U$ est fini sur $\eta$, on a $\bar{U} \simeq U_{\bar{\eta}}$. La construction de (7.24.1) est immédiate dans ce cas puisque le morphisme composé

$$
\dot{\mathbf{E}} \mathbf{t}_{\mathrm{coh} / \bar{\eta}} \stackrel{b^{+}}{\rightarrow} \dot{\mathbf{E}}_{\mathrm{scoh} / \bar{S}} \rightarrow \dot{\mathbf{E}} \mathbf{t}_{\mathrm{coh} / \bar{\eta}},
$$

où la seconde flèche est déduite du changement de base, est l'identité. De plus, on a $\bar{U} \times_{b^{+}\left(U_{\bar{\eta}}\right)} b^{+}(V) \simeq U_{\bar{\eta}} \times_{U_{\bar{\eta}}} V=V$ et $\bar{U}^{V} \simeq V$; d'où l'isomorphisme (7.24.2) dans ce cas.

7.25. On déduit de (7.24.1) un morphisme de foncteurs

$$
t^{+}: a^{+} \rightarrow b^{+} h_{S}^{+}
$$

et donc un 2-morphisme

$$
t: h_{S} b \rightarrow a
$$

D'après la propriété universelle des topos co-évanescents [4, VI.3.7], le triplet $(a, b, t)$ induit un morphisme de topos

$$
u: \bar{S}_{\text {ét }} \rightarrow S_{\text {ét }} \overleftarrow{\times}_{S_{\text {ét }}} \bar{\eta}_{\text {ét }}
$$


qui s’insère dans le diagramme commutatif

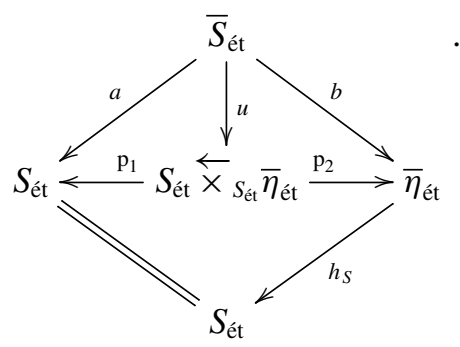

On vérifie aussitôt que

$$
u(\bar{\eta})=(\mathrm{id}: \bar{\eta} \rightsquigarrow \bar{\eta}) \quad \text { et } \quad u(s)=(\bar{\eta} \rightsquigarrow s),
$$

où $\bar{\eta} \rightsquigarrow s$ est l'unique flèche de spécialisation de $\bar{\eta}$ vers $s$. En vertu de [4, VI.3.7.2], $u$ est induit par le foncteur

$$
u^{+}: D_{\mathrm{scoh}} \rightarrow \mathbf{E} \mathbf{t}_{\mathrm{scoh} / \bar{S}} \quad(V \rightarrow U) \mapsto \bar{U} \times_{b^{+}\left(U_{\bar{\eta}}\right)} b^{+}(V),
$$

où la flèche $\bar{U} \rightarrow b^{+}\left(U_{\bar{\eta}}\right)$ est définie dans (7.24.1). On en déduit que $u^{*}((\bar{\eta} \rightarrow$ $\left.\eta)^{a}\right)=\bar{\eta}$. Le topos $\eta_{\text {ét }} \overleftarrow{\times}_{\eta_{\text {et }}} \bar{\eta}_{\text {ét }}$ est canoniquement isomorphe au topos localisé de

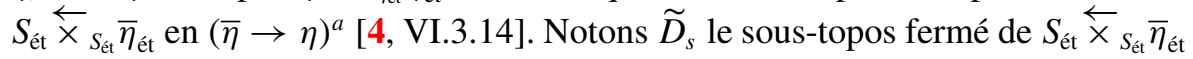
complémentaire de l'ouvert $(\bar{\eta} \rightarrow \eta)^{a}$. Le morphisme $u$ (7.25.2) induit deux morphismes de topos [6, IV 5.11 et 9.4.3]

$$
v: \bar{\eta}_{\text {ét }} \rightarrow \eta_{\text {ét }} \overleftarrow{\times}_{\eta_{\text {et }}} \bar{\eta}_{\text {ét }} \quad \text { et } \quad u_{s}: s_{\text {ét }} \rightarrow \widetilde{D}_{s}
$$

On identifie le morphisme $v$ (resp. $u_{s}$ ) au point (id $\left.: \bar{\eta} \rightsquigarrow \bar{\eta}\right)($ resp. $(\bar{\eta} \rightsquigarrow s)$ ) de $\widetilde{D}$.

Proposition 7.26. Le morphisme u (7.25.2) est une équivalence de topos.

Preuve. D'après [28, 2.3.2], le topos $S_{\text {et }} \overleftarrow{x}_{S_{\text {et }}} \bar{\eta}_{\text {ét }}$ est local de centre $(\bar{\eta} \rightsquigarrow s)[6$ VI 8.4.6]. En vertu de (7.25.4), le morphisme de topos $u$ est local [28, 2.1]. La proposition résulte alors de $7.27,7.29$ et 7.30 ci-dessous.

LEMME 7.27. Le morphisme v (7.25.6) est une équivalence de topos.

Preuve. On désigne par $D_{\text {scoh }}^{\prime}$ le site défini par la sous-catégorie pleine $\left(D_{\text {scoh }}\right)_{/(\bar{\eta} \rightarrow \eta)}$ de $D_{\text {scoh }}$ munie de la topologie induite via le foncteur d'inclusion. En vertu de [4, VI.10.14], le topos $\eta_{\text {ét }} \overleftarrow{x}_{\eta_{\text {ett }}} \bar{\eta}_{\text {ét }}$ est canoniquement isomorphe au 
topos des faisceaux d'ensembles sur $D_{\text {scoh }}^{\prime}$. D'après (7.25.5), le morphisme $v$ est induit par le foncteur

$$
v^{+}: D_{\mathrm{scoh}}^{\prime} \rightarrow \mathbf{E}_{\mathrm{coh} / \bar{\eta}} \quad(V \rightarrow U) \mapsto V .
$$

On désigne par $\mathscr{C}_{\bar{\eta}}$ la catégorie des voisinages du point $v$ de $\eta_{\text {ét }} \overleftarrow{x}_{\eta_{\text {ét }}} \bar{\eta}_{\text {ét }}$ dans le site $D_{\text {scoh }}^{\prime}$. Les objets de $\mathscr{C}_{\bar{\eta}}$ sont les couples formés d'un objet $(V \rightarrow U)$ de $D_{\text {scoh }}^{\prime}$ et d'un $\bar{\eta}$-morphisme $m$ de $\bar{\eta}$ dans $V$. On désigne par $\mathscr{D}_{\bar{\eta}}$ la sous-catégorie pleine de $\mathscr{C}_{\bar{\eta}}$ formée des objets de la forme $\left(\bar{\eta} \rightarrow U, \mathrm{id}_{\bar{\eta}}\right)$. Il est clair que $\mathscr{D}_{\bar{\eta}}^{\circ}$ forme une sous-catégorie cofinale de $\mathscr{C}_{\bar{\eta}}^{\circ}$.

Pour tout faisceau $F$ de $\eta_{\text {ét }} \overleftarrow{\times}_{\eta_{\text {et }}} \bar{\eta}_{\text {ét }}$, considérons le morphisme d'adjonction

$$
v^{*}(F) \rightarrow v^{*} v_{*} v^{*}(F) .
$$

Celui-là s’identifie à la limite inductive sur la catégorie $\mathscr{D}_{\bar{\eta}}^{\circ}$ des morphismes

$$
F(\bar{\eta} \rightarrow U) \rightarrow v_{*}\left(v^{*}(F)\right)(\bar{\eta} \rightarrow U) .
$$

D'après (7.27.1), on a un isomorphisme $v_{*}\left(v^{*}(F)\right)(\bar{\eta} \rightarrow U) \simeq v^{*}(F)(\bar{\eta})=$ $v^{*}(F)$. Le morphisme (7.27.3) s'identifie alors au morphisme canonique $F(\bar{\eta} \rightarrow$ $U) \rightarrow v^{*}(F)$. En prenant la limite inductive, le morphisme (7.27.2) est donc un isomorphisme. Comme le point $v$ est conservatif pour le topos $\eta_{\text {ét }} \overleftarrow{\times}_{\eta_{\text {êt }}} \bar{\eta}_{\text {ét }}$ [4, VI.5.28], on en déduit que le morphisme d'adjonction

$$
\mathrm{id} \rightarrow v_{*} v^{*}
$$

est un isomorphisme. Celui-là implique que $v^{*}$ est pleinement fidèle [6, I 6.4]. Il est clair que le foncteur fibre $v^{*}$ est essentiellement surjectif, d'où le lemme.

Lemme 7.28. Soit $\mathcal{T}$ un topos local de point central $s: \mathbf{P t} \rightarrow \mathcal{T}$. On suppose que le point s est conservatif pour $\mathcal{T}$. Alors, s est une équivalence de topos.

Preuve. Notons $\varepsilon: \mathcal{T} \rightarrow$ Pt la projection canonique [6, IV 4.3]. Rappelons que, pour tout faisceau $F$ de $\mathcal{T}$, le morphisme naturel $\Gamma(\mathcal{T}, F) \rightarrow s^{*}(F)$ est bijectif. Celui-là induit un isomorphisme canonique $\varepsilon_{*} \stackrel{\sim}{\rightarrow} s^{*}$. On en déduit un isomorphisme

$$
\varepsilon_{*} S_{*} \stackrel{\sim}{\rightarrow} s^{*} s_{*} .
$$

On notera que le morphisme composé $\varepsilon \circ s: \mathbf{P t} \rightarrow \mathcal{T} \rightarrow$ Pt s'identifie au morphisme identique. On en déduit que le composé de (7.28.1) et du morphisme d'adjonction

$$
\varepsilon_{*} s_{*} \stackrel{\sim}{\rightarrow} s^{*} s_{*} \rightarrow \mathrm{id}
$$


s'identifie au morphisme identique. Par suite, le morphisme d'adjonction

$$
s^{*} s_{*} \stackrel{\sim}{\rightarrow} \mathrm{id}
$$

est un isomorphisme. D'autre part, le morphisme composé déduit des morphismes d'adjonction $s^{*} \rightarrow s^{*} s_{*} s^{*} \rightarrow s^{*} s^{\prime}$ 'identifie au morphisme identique [19, 1.1.12]. On en déduit un isomorphisme canonique

$$
s^{*} \stackrel{\sim}{\rightarrow} s^{*} s_{*} s^{*} .
$$

Comme le foncteur fibre $s^{*}$ est conservatif, le morphisme d'adjonction

$$
\mathrm{id} \stackrel{\sim}{\rightarrow} s_{*} s^{*}
$$

est un isomorphisme. Le morphisme $s$ induit donc une équivalence de topos.

LEMME 7.29. Le morphisme $u_{s}$ (7.25.6) est une équivalence de topos.

Preuve. Les points $v$ et $u_{s}$ sont conservatifs pour le topos $S_{\text {ét }} \overleftarrow{\times}_{S_{\text {et }}} \bar{\eta}_{\text {ét }}\left(\underset{D_{s}}{\text { (cf. 7.6). Par }}\right.$ 7.27 et [6, IV 9.7.3], on en déduit que le point $u_{s}$ est conservatif pour $\widetilde{D}_{s}$. D'après 7.28 , il suffit de démontrer que le topos $\widetilde{D}_{s}$ est local de centre $u_{s}$. Considérons le morphisme composé de topos

$$
s_{\text {ét }} \stackrel{u_{s}}{\longrightarrow} \widetilde{D}_{s} \stackrel{i}{\longrightarrow} S_{\text {ét }} \overleftarrow{\times}_{S_{\mathrm{et}}} \bar{\eta}_{\text {ét }} \stackrel{\varepsilon}{\longrightarrow} \mathbf{P t}
$$

où $i$ est le morphisme d'injection canonique et $\varepsilon$ la projection canonique. Rappelons [6, IV 9.5.8] que le morphisme d'adjonction

$$
i^{*} i_{*} \stackrel{\sim}{\rightarrow} \mathrm{id}
$$

est un isomorphisme. Comme $S_{\text {ét }} \overleftarrow{x}_{S_{\text {et }}} \bar{\eta}_{\text {ét }}$ est local de centre $i \circ u_{s}(7.25 .4)$, on a un isomorphisme canonique

$$
\varepsilon_{*} \stackrel{\sim}{\rightarrow} u_{s}^{*} i^{*}
$$

On en déduit que le composé

$$
\varepsilon_{*} i_{*} \rightarrow u_{s}^{*} i^{*} i_{*} \rightarrow u_{s}^{*}
$$

est un isomorphisme. Le foncteur 'sections globales' $\Gamma\left(\widetilde{D}_{s},-\right)$ est donc isomorphe au foncteur fibre $u_{s}^{*}$, i.e. le topos $\widetilde{D}_{s}$ est local de centre $u_{s}$.

LEMME 7.30. Soit $f: \mathcal{T}^{\prime} \rightarrow \mathcal{T}$ un morphisme de topos, $U$ un ouvert de $\mathcal{T}$ et $U^{\prime}=f^{*}(U)$. Notons $j: \mathcal{T}_{/ U} \rightarrow \mathcal{T}$ (resp. $j^{\prime}: \mathcal{T}_{/ U^{\prime}}^{\prime} \rightarrow \mathcal{T}^{\prime}$ ) le morphisme de 
localisation de $\mathcal{T}$ (resp. $\mathcal{T}^{\prime}$ ) en $U\left(\right.$ resp. $\left.U^{\prime}\right)$ et $i: \mathcal{T}_{s} \rightarrow \mathcal{T}\left(\right.$ resp. $\left.i^{\prime}: \mathcal{T}_{s}^{\prime} \rightarrow \mathcal{T}^{\prime}\right)$ le sous-topos fermé de $\mathcal{T}$ (resp. $\mathcal{T}^{\prime}$ ) complémentaire de l'ouvert $U$ (resp. $U^{\prime}$ ) [6, IV 9.3.5]. Le morphisme $f$ induit deux morphismes de topos [6, IV 5.11 et 9.4.3]

$$
f_{/ U}: \mathcal{T}_{/ U^{\prime}}^{\prime} \rightarrow \mathcal{T}_{/ U} \quad \text { et } \quad f_{s}: \mathcal{T}_{s}^{\prime} \rightarrow \mathcal{T}_{s}
$$

Supposons que $\mathcal{T}_{s}$ (resp. $\mathcal{T}_{s}^{\prime}$ ) soit ponctuel, $\mathcal{T}$ (resp. $\mathcal{T}^{\prime}$ ) soit local de centre $i$ (resp. $\left.i^{\prime}\right)$ et que $f_{/ U}$ soit une équivalence de topos. Alors, $f$ est une équivalence de topos.

Preuve. Posons $\rho^{\prime}=i^{\prime *} \circ j_{*}^{\prime}$ et $\rho=i^{*} \circ j_{*}$. Le topos $\mathcal{T}$ (resp. $\mathcal{T}^{\prime}$ ) est équivalent au topos obtenu par recollement de $\left(\mathcal{T}_{/ U}, \mathcal{T}_{s}, \rho\right)\left(\operatorname{resp} .\left(\mathcal{T}_{/ U^{\prime}}^{\prime}, \mathcal{T}_{s}^{\prime}, \rho^{\prime}\right)\right)(\mathrm{cf}$. [6, IV 9.5.4]). Considérons le diagramme

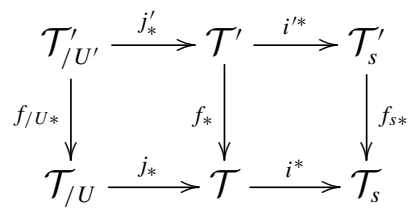

dont le carré de gauche est clairement commutatif. Le foncteur $i^{*}$ (resp. $i^{\prime *}$ ) est isomorphe au foncteur 'sections globales' $\Gamma(\mathcal{T},-)\left(\operatorname{resp} . \Gamma\left(\mathcal{T}^{\prime},-\right)\right)$ et le foncteur $f_{s *}$ est isomorphe au foncteur identique. On en déduit que le carré de droite de (7.30.2) est aussi commutatif. Les foncteurs $\rho$ et $\rho^{\prime}$ sont donc compatibles. Par suite, $f_{*}$ induit une équivalence de catégories en vertu de [6, IV 9.5.2].

7.31. Soit $\overline{\mathscr{B}}_{p t}$ l'anneau de $\widetilde{E}_{p t}$ associé à $\bar{S}$ (7.8). Par (7.8.1), (7.24.2) et (7.25.5), on a un isomorphisme d'anneaux de $\widetilde{E}_{p t}$

$$
\rho_{*} u_{*}\left(\mathscr{O}_{\bar{S}}\right) \stackrel{\sim}{\rightarrow} \overline{\mathscr{B}}_{p t} .
$$

Par les équivalences de topos (7.22.1) et (7.25.2):

$$
\bar{S}_{\text {ét }} \stackrel{u}{\rightarrow} S_{\text {ét }} \overleftarrow{\times}_{S_{\text {et }}} \bar{\eta}_{\text {ét }} \stackrel{\rho}{\rightarrow} \widetilde{E}_{p t}
$$

l'anneau $\mathscr{O}_{\bar{S}}$ de $\bar{S}_{\text {ét }}$ s'identifie alors à l'anneau $\overline{\mathscr{B}}_{p t}$ de $\widetilde{E}_{p t}$ et pour tout entier $n \geqslant 1$, l'anneau $\mathscr{O}_{\bar{S}_{n}}$ de $\bar{S}_{\text {ét }}$ s'identifie alors à l'anneau $\overline{\mathscr{B}}_{p t, n}$ de $\widetilde{E}_{p t}$ (7.8.3). D'après (7.22.2) et 7.29, le sous-topos fermé $s_{\text {ét }}$ de $\bar{S}_{\text {ét }}$ s'identifie au sous-topos fermé $\widetilde{E}_{p t, s}(7.10 .1)$ de $\widetilde{E}_{p t}$.

D'après (7.22.2) et (7.25.3), le morphisme de topos $a: \bar{S}_{\text {ét }} \rightarrow S_{\text {ét }}$ induit une équivalence de topos annelés

$$
a_{n}:\left(s_{\text {ét }}, \mathscr{O}_{\bar{S}_{n}}\right) \stackrel{\sim}{\rightarrow}\left(s_{\text {ét }}, \mathscr{O}_{\bar{S}_{n}}\right),
$$


qui s'identifie au morphisme de topos annelés $\sigma_{n}$ (7.11.4) de $\widetilde{E}_{p t}$. De plus, le morphisme de topos $b: \bar{S}_{\text {ét }} \rightarrow \bar{\eta}_{\text {fét }}$ induit une équivalence de topos annelés

$$
b_{n}:\left(s_{\text {ét }}, \mathscr{O}_{\bar{S}_{n}}\right) \stackrel{\sim}{\rightarrow}\left(\bar{\eta}_{\text {fét }}, \mathfrak{o}_{n}\right)
$$

qui s'identifie au morphisme de topos annelés $\beta_{n}$ (7.13.1) de $\widetilde{E}_{p t}$.

\section{Correspondance de Deninger-Werner via le topos annelé de Faltings}

Dans cette section, on se donne une $S$-courbe semi-stable $X$ et un $\bar{\eta}$-point $\bar{x}$ de $X_{\bar{\eta}}$. On pose $C=X_{\bar{\eta}}$. Rappelons que $\bar{X}=X \times{ }_{S} \bar{S}$ est un schéma normal (cf. 5.6). On reprend les notations de la Section 7 pour $X$.

8.1. Pour tout $\bar{\eta}$-point $\bar{y}$ de $C$, il existe un trait $S^{\prime}$ fini sur $S$, un morphisme $h_{S^{\prime}}: \bar{\eta} \rightarrow S^{\prime}$ et un $S$-morphisme $y: S^{\prime} \rightarrow X$ qui s'insèrent dans un diagramme commutatif de schémas

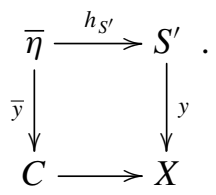

On identifie le topos annelé $\left(\bar{S}_{\text {ét }}, \mathscr{O}_{\bar{S}}\right)$ au topos annelé de Faltings associé au morphisme $h_{S^{\prime}}$ et au schéma $\bar{S}$ (cf. 7.31). Pour tout entier $n \geqslant 1$, le diagramme (8.1.1) induit par fonctorialité [4, III.8.20] un morphisme de topos annelés que l'on note

$$
[\bar{y}]_{n}:\left(s_{\text {ét }}, \mathscr{O}_{\bar{S}_{n}}\right) \rightarrow\left(\widetilde{E}_{s}, \overline{\mathscr{B}}_{n}\right) .
$$

Par fonctorialité du topos de Faltings [4, VI.10.12], celui-ci est indépendant des choix du trait $S^{\prime}$ fini sur $S$ et du diagramme (8.1.1).

Proposition 8.2. Supposons $X$ régulier. Soit $n$ un entier $\geqslant 1, M$ un $\overline{\mathscr{B}}_{n}$-module libre de type fini et $\bar{y}$ un point géométrique de C. Le morphisme $[\bar{y}]_{n}(8.1 .2)$ et le foncteur $(-)_{\sharp}(3.1 .1)$ induisent un isomorphisme fonctoriel

$$
\left([\bar{y}]_{n}^{*}\right)_{\sharp}: \Gamma\left(\widetilde{E}_{s}, M\right)_{\sharp} \stackrel{\sim}{\rightarrow} \Gamma\left(s_{\text {ét }},[\bar{y}]_{n}^{*}(M)\right)_{\sharp} .
$$

Preuve. Comme $M$ est libre de type fini, il existe un $\mathfrak{o}_{n}$-module libre de type fini $\mathbb{L}$ de $C_{\text {fét }}$ tel que $M \simeq \beta_{n}^{*}(\mathbb{L})$. D'après [4, VI.(10.12.6)], on a un diagramme 
commutatif de topos annelés

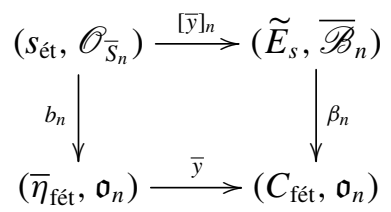

où $b_{n}$ est l'équivalence de topos annelés (7.31.4), induisant un diagramme commutatif :

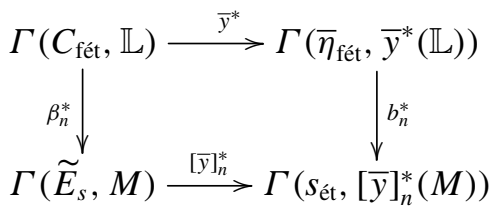

où $\beta_{n}^{*}$ induit un $\alpha$-isomorphisme en vertu de 7.20 et $\bar{y}^{*}$ induit un isomorphisme puisque $\mathbb{L}_{\text {est }}$ un faisceau constant et $C$ connexe. On en déduit un $\alpha$-isomorphisme

$$
[\bar{y}]_{n}^{*}: \Gamma\left(\widetilde{E}_{s}, M\right) \rightarrow \Gamma\left(s_{\text {ét }},[\bar{y}]_{n}^{*}(M)\right) .
$$

L'isomorphisme (8.2.1) résulte de 3.2(i).

8.3. Soit $\left(S^{\prime}, \eta^{\prime}\right)$ un trait fini sur $(S, \eta), \varphi: X^{\prime} \rightarrow X_{S^{\prime}}$ un $\eta^{\prime}$-revêtement semistable. On note encore $s$ le point fermé de $S^{\prime}$ et on fixe un $\eta$-morphisme $\bar{\eta} \rightarrow \eta^{\prime}$ et par suite un $S$-morphisme $\bar{S} \rightarrow S^{\prime}$. On pose $X_{s}^{\prime}=X^{\prime} \times_{S^{\prime}} s, \vec{X}{ }^{\prime}=X^{\prime} \times_{S^{\prime}} \bar{S}$ et $C^{\prime}=X^{\prime} \times{ }_{S^{\prime}} \bar{\eta}$. On désigne par

$$
\phi: X^{\prime} \stackrel{\varphi}{\rightarrow} X_{S^{\prime}} \rightarrow X, \bar{\varphi}: \vec{X}^{\prime} \rightarrow \bar{X}, \hbar^{\prime}: \vec{X}^{\prime} \rightarrow X^{\prime} \text { et } h^{\prime}: C^{\prime} \rightarrow X^{\prime}
$$

les morphismes canoniques. On a un diagramme commutatif induit par $\varphi$

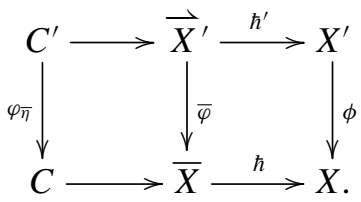

Comme $X^{\prime}$ est une $S^{\prime}$-courbe semi-stable, le schéma $\vec{X}^{\prime}$ est normal (5.6). On désigne par $\left(\widetilde{E}^{\prime}, \overline{\mathscr{B}}^{\prime}\right)$ le topos annelé de Faltings associé au schéma $X^{\prime}$ au-dessus de $S^{\prime}$ (cf. 7.1 et 7.8) et par

$$
\Phi:\left(\widetilde{E}^{\prime}, \overline{\mathscr{B}}^{\prime}\right) \rightarrow(\widetilde{E}, \overline{\mathscr{B}})
$$


le morphisme induit par fonctorialité par (8.3.2) [4, III.8.20]. D'après [4, VI.(10.12.6)], les carrés du diagramme

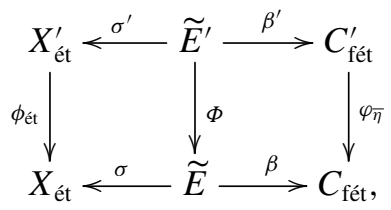

où $\beta^{\prime}$ et $\sigma^{\prime}$ sont les morphismes canoniques (7.3.2) et (7.3.4) relatifs au $S^{\prime}$-schéma $X^{\prime}$, sont commutatifs à isomorphismes canoniques près.

On note $\widetilde{E}_{s}^{\prime}$ le sous-topos fermé de $\widetilde{E}^{\prime}$ complémentaire de l'ouvert $\sigma^{\prime *}\left(X_{\eta^{\prime}}^{\prime}\right)$ (7.10),

$$
\delta^{\prime}: \widetilde{E}_{s}^{\prime} \rightarrow \widetilde{E}^{\prime}
$$

le plongement canonique (7.10.1) et

$$
\sigma_{s}^{\prime}: \widetilde{E}_{s}^{\prime} \rightarrow X_{s, \text { ét }}^{\prime}
$$

le morphisme canonique de topos (7.11.1). D'après (8.3.4), on a un isomorphisme $\Phi^{*}\left(\sigma^{*}\left(X_{\eta}\right)\right) \simeq \sigma^{*}\left(X_{\eta^{\prime}}^{\prime}\right)$. En vertu de [6, IV 9.4.3], il existe donc un morphisme de topos

$$
\Phi_{s}: \widetilde{E}_{s}^{\prime} \rightarrow \widetilde{E}_{s}
$$

unique à isomorphisme près tel que le diagramme

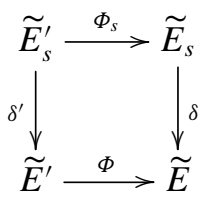

soit commutatif. Il résulte de (8.3.4) et de [6, IV 9.4.3] que le diagramme de morphismes de topos

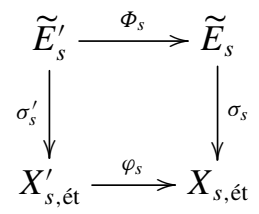

est commutatif à isomorphisme près.

Pour tout entier $n \geqslant 1$, on pose $\overline{\mathscr{B}}_{n}^{\prime}=\overline{\mathscr{B}}^{\prime} / p^{n} \overline{\mathscr{B}}^{\prime}$ et l'on désigne par

$$
\sigma_{n}^{\prime}:\left(\widetilde{E}_{s}^{\prime}, \overline{\mathscr{B}}_{n}^{\prime}\right) \rightarrow\left(X_{s, \text { ét }}^{\prime}, \mathscr{O}_{\vec{X}_{n}^{\prime}}\right)
$$


le morphisme de topos annelés induit par $\sigma^{\prime}(7.11 .4)$ et par

$$
\beta_{n}^{\prime}:\left(\widetilde{E}_{s}^{\prime}, \overline{\mathscr{B}}_{n}^{\prime}\right) \rightarrow\left(C_{\text {fét }}^{\prime}, \mathfrak{o}_{n}\right)
$$

le morphisme de topos annelés induit par $\beta^{\prime}$ (7.13.1). L'homomorphisme canonique $\Phi^{-1}(\overline{\mathscr{B}}) \rightarrow \overline{\mathscr{B}}^{\prime}$ induit un homomorphisme $\Phi_{s}^{-1}\left(\overline{\mathscr{B}}_{n}\right) \rightarrow \overline{\mathscr{B}}_{n}^{\prime}$. Le morphisme $\Phi_{s}$ est donc sous-jacent à un morphisme de topos annelés que l'on note

$$
\Phi_{n}:\left(\widetilde{E}_{s}^{\prime}, \overline{\mathscr{B}}_{n}^{\prime}\right) \rightarrow\left(\widetilde{E}_{s}, \overline{\mathscr{B}}_{s}\right) .
$$

Il résulte de (8.3.9) et de la définition de (7.11.3) que le diagramme de morphismes de topos annelés

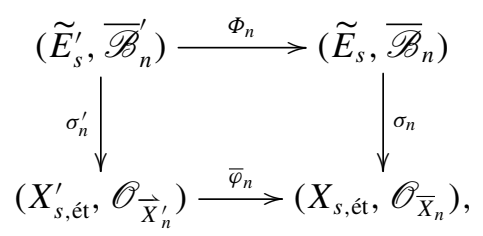

où $\bar{\varphi}_{n}$ est la réduction modulo $p^{n}$ de $\bar{\varphi}$, est commutatif à isomorphisme près. D'après (8.3.4), (8.3.8) et la définition de (7.13.1), le diagramme de morphismes de topos annelés

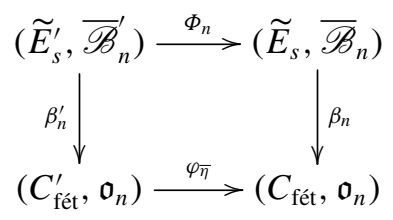

est commutatif à isomorphisme près.

Les morphismes $\left(\Phi_{n}\right)_{n \geqslant 1}$ définissent un morphisme de topos annelés

$$
\breve{\Phi}:\left(\widetilde{E}_{s}^{\mathbb{N}^{\circ}}, \breve{\mathscr{B}}^{\prime}\right) \rightarrow\left(\widetilde{E}_{s}^{\mathbb{N}^{\circ}}, \breve{\mathscr{B}}\right) .
$$

8.4. Conservons les notations de 8.3 et supposons de plus que le $\eta^{\prime}$-revêtement semi-stable $\varphi: X^{\prime} \rightarrow X_{S^{\prime}}$ soit galoisien. Posons $G=\operatorname{Aut}\left(X_{\eta^{\prime}}^{\prime} / X_{\eta^{\prime}}\right)$, qui est isomorphe à $\operatorname{Aut}\left(C^{\prime} / C\right)$. Tout $g \in G$ s'étend en un $X$-automorphisme de $X^{\prime}$. Par suite, celui-là induit, pour tout entier $n \geqslant 1$, un morphisme de topos annelés $g_{n}:\left(\widetilde{E}_{s}^{\prime}, \overline{\mathscr{B}}_{n}^{\prime}\right) \rightarrow\left(\widetilde{E}_{s}^{\prime}, \overline{\mathscr{B}}_{n}^{\prime}\right)$ tel que $\Phi_{n} \simeq \Phi_{n} \circ g_{n}$ et que pour tous $g, h \in G$, on ait un isomorphisme

$$
(g h)_{n}^{*} \simeq h_{n}^{*} \circ g_{n}^{*}
$$

DÉFINITION 8.5. (i) On dit qu'un $\overline{\mathscr{B}}_{n}$-module $M_{n}$ est potentiellement libre de type fini s'il est de type fini, et s'il existe un trait $\left(S^{\prime}, \eta^{\prime}\right)$ fini $\operatorname{sur}(S, \eta)$ et 
un $\eta^{\prime}$-revêtement semi-stable $\varphi: X^{\prime} \rightarrow X_{S^{\prime}}$ tel que, reprenant les notations de 8.3, $\Phi_{n}^{*}\left(M_{n}\right)$ soit un $\overline{\mathscr{B}}_{n}^{\prime}$-module libre de type fini.

(ii) On dit qu'un $\breve{\mathscr{B}}$-module $M=\left(M_{n}\right)_{n} \geqslant 1$ est potentiellement libre de type fini si $M$ est adique de type fini (2.11) et si, pour tout entier $n \geqslant 1$, le $\overline{\mathscr{B}}_{n}$-module $M_{n}$ est potentiellement libre de type fini.

On désigne par $\operatorname{Mod}^{\mathrm{pltf}}\left(\overline{\mathscr{B}}_{n}\right)$ (resp. $\operatorname{Mod}^{\mathrm{pltf}}(\breve{\mathscr{B}})$ ) la sous-catégorie pleine de $\operatorname{Mod}\left(\overline{\mathscr{B}}_{n}\right)(\operatorname{resp} . \operatorname{Mod}(\breve{\mathscr{B}}))$, formée des $\overline{\mathscr{B}}_{n}$-modules potentiellement libres de type fini (resp. $\breve{\mathscr{B}}$-modules potentiellement libres de type fini). On désigne par $\operatorname{Mod}_{\mathbb{Q}}^{\text {plff }}(\breve{\mathscr{B}})$ la catégorie des objets de $\operatorname{Mod}^{\text {pltf }}(\breve{\mathscr{B}})$ à isogénie près; c'est une souscatégorie pleine de $\operatorname{Mod}_{\mathbb{Q}}^{\text {atf }}(\breve{\mathscr{B}})(7.15)$. Les objets de $\operatorname{Mod}_{\mathbb{Q}}^{\text {pltf }}(\breve{\mathscr{B}})$ sont appelés les $\overline{\mathscr{B}}_{\mathbb{Q}}$-modules potentiellement libres de type fini.

8.6. Dans cette section, on reprend les notations de 3.21 pour le groupe profini $\pi_{1}(C, \bar{x})$. Soit $n \geqslant 1$ un entier. On note abusivement $\beta_{n}^{*}, \breve{\beta}^{*}$ et $\breve{\beta}_{\mathbb{Q}}^{*}$ les foncteurs composés suivants :

$$
\begin{aligned}
& \beta_{n}^{*}: \operatorname{Rep}_{\mathfrak{o}_{n}}^{\text {lff }}\left(\pi_{1}(C, \bar{x})\right) \stackrel{\mu_{\bar{x}}}{\rightarrow} \mathbf{L L}^{\mathrm{tf}}\left(C_{\mathrm{fét}}, \mathfrak{o}_{n}\right) \stackrel{\beta_{n}^{*}}{\rightarrow} \operatorname{Mod}\left(\overline{\mathscr{B}}_{n}\right), \\
& \breve{\beta}^{*}: \operatorname{Rep}_{\mathfrak{o}}^{\text {lff }}\left(\pi_{1}(C, \bar{x})\right) \stackrel{\breve{\mu}_{\bar{x}}}{\rightarrow} \mathbf{L L}^{\mathrm{tf}}\left(C_{\text {fét }}^{\mathbb{N}^{\circ}}, \breve{\mathfrak{o}}\right) \stackrel{\breve{\beta}^{*}}{\rightarrow} \operatorname{Mod}(\stackrel{\breve{\mathscr{B}}}{\rightarrow}, \\
& \breve{\beta}_{\mathbb{Q}}^{*}: \operatorname{Rep}_{\mathfrak{C}}^{\text {cont }}\left(\pi_{1}(C, \bar{x})\right) \stackrel{\breve{\mu}_{\bar{x}, \mathbb{Q}}}{\longrightarrow} \mathbf{L L}_{\mathbb{Q}}^{\mathrm{tf}}\left(C_{\mathrm{fét}}^{\mathbb{N}^{\circ}}, \breve{\mathfrak{o}}\right) \stackrel{\breve{\beta}_{\mathbb{Q}}^{*}}{\longrightarrow} \operatorname{Mod}_{\mathbb{Q}}(\stackrel{\breve{\mathscr{B}}}{\longrightarrow}),
\end{aligned}
$$

où $\mu_{\bar{x}}, \breve{\mu}_{\bar{x}}$ et $\breve{\mu}_{\bar{x}, \mathbb{Q}}$ sont les équivalences de catégories définies dans 3.29.

Proposition 8.7. Soit $n$ un entier $\geqslant 1$. Le foncteur $\beta_{n}^{*}\left(\right.$ resp. $\breve{\beta}^{*}$, resp. $\left.\breve{\beta}_{\mathbb{Q}}^{*}\right)$ est exact et il se factorise à travers la sous-catégorie $\operatorname{Mod}^{\mathrm{pltf}}\left(\overline{\mathscr{B}}_{n}\right)\left(\operatorname{resp} \cdot \operatorname{Mod}^{\mathrm{pltf}}(\breve{\mathscr{B}})\right.$, resp. $\left.\operatorname{Mod}_{\mathbb{Q}}^{\text {pltf }}(\breve{\mathscr{B}})\right)$.

Preuve. L'anneau $\overline{\mathscr{B}}$ est plat sur $\mathcal{O}_{\bar{K}}\left[4\right.$, III.9.2] et par suite, $\overline{\mathscr{B}}_{n}$ est une $\mathfrak{o}_{n}$-algèbre plate, d'où l'exactitude du foncteur $\beta_{n}^{*}$. On en déduit que les foncteurs $\breve{\beta}^{*}$ et $\breve{\beta}_{\mathbb{Q}}^{*}$ sont exacts.

Étant donné un objet $\mathbb{L}$ de $\mathbf{L} \mathbf{L}^{\mathrm{tf}}\left(C_{\text {fét }}, \mathfrak{o}_{n}\right)$, il existe un revêtement étale, connexe et galoisien $\phi: C^{\prime} \rightarrow C$ tel que $\phi^{*}(\mathbb{L})$ soit un $\mathfrak{o}_{n}$-module libre de type fini de $C_{\text {fét. }}^{\prime}$ D'après 5.11(i), quitte à remplacer $S$ par une extension finie, il existe un $\eta$-revêtement semi-stable $\varphi: X^{\prime} \rightarrow X$ de fibre géométrique générique $\phi:$ $C^{\prime} \rightarrow C$. Reprenons les notations de 8.3 dans ce cas. D'après (8.3.14), on a un 
isomorphisme

$$
\Phi_{n}^{*}\left(\beta_{n}^{*}(\mathbb{L})\right) \simeq \beta_{n}^{\prime *}\left(\varphi_{\bar{\eta}}^{*}(\mathbb{L})\right) .
$$

Par suite, $\beta_{n}^{*}(\mathbb{L})$ est potentiellement libre de type fini. L'assertion pour le foncteur $\breve{\beta}^{*}$ s'ensuit compte tenu de 3.28(ii).

PROPOSITION 8.8. Supposons X régulier. Le foncteur (8.6.3) est pleinement fidèle.

Preuve. Étant donné deux objets $\mathbb{L}_{1}, \mathbb{L}_{2}$ de $\mathbf{L} \mathbf{L}^{\mathrm{tf}}\left(C_{\text {fét }}^{\mathbb{N}^{\circ}}, \breve{\mathfrak{o}}\right)$, on pose

$$
\mathbb{L}=\mathscr{H}_{o m_{\mathfrak{o}}}\left(\mathbb{L}_{1}, \mathbb{L}_{2}\right),
$$

qui est encore un $\breve{\mathfrak{o}}$-module localement libre de type fini de $C_{\text {fét }}^{\mathbb{N}}$. Comme le $\breve{\mathfrak{o}}$ module $\mathbb{L}_{1}$ est localement libre de type fini, on a un isomorphisme canonique

$$
\left.\breve{\beta}^{*}(\mathbb{L}) \stackrel{\sim}{\rightarrow} \mathscr{H}_{o m} \breve{\breve{B}}_{\left(\bar{\beta}^{*}\right.}\left(\mathbb{L}_{1}\right), \breve{\beta}^{*}\left(\mathbb{L}_{2}\right)\right)
$$

D'après 7.21 et (8.8.1), le foncteur $\breve{\beta}$ induit un isomorphisme :

$$
\operatorname{Hom}_{\breve{\mathfrak{o}}}\left(\mathbb{L}_{1}, \mathbb{L}_{2}\right) \otimes_{\mathbb{Z}} \mathbb{Q} \stackrel{\sim}{\rightarrow} \operatorname{Hom}_{\breve{\mathscr{B}}}\left(\breve{\beta}^{*}\left(\mathbb{L}_{1}\right), \breve{\beta}^{*}\left(\mathbb{L}_{2}\right)\right) \otimes_{\mathbb{Z}} \mathbb{Q} ;
$$

d'où la pleine fidélité de (8.6.3).

LEMME 8.9. Soit $M$ un $\overline{\mathscr{B}}_{n}$-module potentiellement libre de type fini. Le $\mathfrak{o}_{n}$ module

$$
\mathscr{W}_{n}(M)=\Gamma\left(s_{\text {ét }},[\bar{x}]_{n}^{*}(M)\right)_{\sharp},
$$

où $[\bar{x}]_{n}:\left(s_{\text {ét }}, \mathscr{O}_{\bar{X}_{n}}\right) \rightarrow\left(\widetilde{E}_{s}, \overline{\mathscr{B}}_{n}\right)$ est le morphisme de topos annelés induit par $\bar{x}$ (8.1.2) et $(-)_{\sharp}$ est le foncteur (3.1.1), est $\alpha$-plat de type $\alpha$-fini $(3.4,3.11)$.

Preuve. D'après 5.11(i), il existe un trait $\left(S^{\prime}, \eta^{\prime}\right)$ fini sur $(S, \eta)$ et un $\eta^{\prime}$ revêtement semi-stable, régulier et galoisien $\varphi: X^{\prime} \rightarrow X_{S^{\prime}}$ tel que, fixant un $\eta$-morphisme $\bar{\eta} \rightarrow \eta^{\prime}$ et reprenant les notations de $8.3, \Phi_{n}^{*}(M)$ soit libre de type fini et que chaque $\bar{\eta}$-point de $C^{\prime}$ au-dessus de $\bar{x}$ se descende en un $\eta^{\prime}$-point de $X_{\eta^{\prime}}^{\prime}$.

Choisissons un $\bar{\eta}$-point $\bar{y}$ de $C^{\prime}$ au-dessus de $\bar{x}$ et un $\eta^{\prime}$-point de $X_{\eta^{\prime}}^{\prime}$ qui le descend. On note $y$ le $S^{\prime}$-point de $X^{\prime}$ correspondant et $x: S^{\prime} \rightarrow X$ le $S$-morphisme induit $\operatorname{par} \varphi$. Le diagramme commutatif de couples de schémas

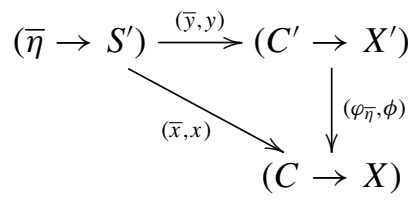


induit un diagramme commutatif de topos annelés :

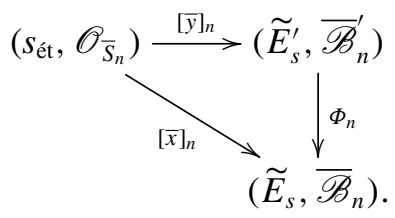

On en déduit un isomorphisme $\mathscr{W}_{n}(M) \simeq \Gamma\left(s_{\text {ét }},[\bar{y}]_{n}^{*}\left(\Phi_{n}^{*}(M)\right)\right)_{\sharp}$. Comme $\Phi_{n}^{*}(M)$ est un $\overline{\mathscr{B}}_{n}^{\prime}$-module libre de type fini, l'assertion s'ensuit compte tenu de 3.14.

8.10. On fixe un entier $\geqslant 1$. Dans la suite de cette section, on se propose de construire un foncteur de $\operatorname{Mod}^{\text {pltf }}\left(\overline{\mathscr{B}}_{n}\right)$ dans $\operatorname{Rep}_{\mathfrak{o}_{n}}^{\alpha \text { ptf }}\left(\pi_{1}(C, \bar{x})\right)$ (3.23). Étant donné un $\overline{\mathscr{B}}_{n}$-module potentiellement libre de type fini $M$, on définit le $\mathfrak{o}_{n}$-module $\mathscr{W}_{n}(M)$ sous-jacent à la $\mathfrak{o}_{n}$-représentation associée à $M$ par (8.9.1). Reprenant les notations de la preuve de 8.9 , le morphisme $[\bar{y}]_{n}$ induit un isomorphisme $\mathfrak{o}_{n^{-}}$ linéaire (cf. 8.2)

$$
\left([\bar{y}]_{n}^{*}\right)_{\sharp}: \Gamma\left(\widetilde{E}_{s}^{\prime}, \Phi_{n}^{*}(M)\right)_{\sharp} \stackrel{\sim}{\rightarrow} \mathscr{W}_{n}(M) .
$$

On construit une action $\varrho_{M}$ de $\pi_{1}(C, \bar{x})$ sur $\mathscr{W}_{n}(M)$ comme suit. Posons $G=\operatorname{Aut}\left(X_{\eta^{\prime}}^{\prime} / X_{\eta^{\prime}}\right)$ qui est isomorphe à $\operatorname{Aut}\left(C^{\prime} / C\right)$. D'après 8.4, tout $g \in G$ induit un morphisme de topos annelés $g_{n}:\left(\widetilde{E}_{s}^{\prime},{\mathscr{\mathscr { B }}_{n}^{\prime}}^{\prime}\right) \rightarrow\left(\widetilde{E}_{s}^{\prime}, \overline{\mathscr{B}}_{n}^{\prime}\right)$ et par suite un automorphisme

$$
\left(g_{n}^{*}\right)_{\sharp}: \Gamma\left(\widetilde{E}_{s}^{\prime}, \Phi_{n}^{*}(M)\right)_{\sharp} \stackrel{\sim}{\rightarrow} \Gamma\left(\widetilde{E}_{s}^{\prime}, \Phi_{n}^{*}(M)\right)_{\sharp} .
$$

On a un homomorphisme surjectif (6.6.2)

$$
\xi_{\bar{y}}: \pi_{1}(C, \bar{x}) \rightarrow G^{\mathrm{op}} .
$$

Pour tout $\gamma \in \pi_{1}(C, \bar{x})$, posant $g=\xi_{\bar{y}}(\gamma)$, on définit un automorphisme $\varrho_{M}(\gamma)$ de $\mathscr{W}_{n}(M)$ par

$$
\begin{gathered}
\varrho_{M}(\gamma): \mathscr{W}_{n}(M) \stackrel{\left(\left([\bar{y}]_{n}^{*}\right)_{\sharp}\right)^{-1}}{\longrightarrow} \Gamma\left(\widetilde{E}_{s}^{\prime}, \Phi_{n}^{*}(M)\right)_{\sharp} \\
\stackrel{\left(g_{n}^{*}\right)_{\sharp}}{\longrightarrow} \Gamma\left(\widetilde{E}_{s}^{\prime}, \Phi_{n}^{*}(M)\right)_{\sharp} \stackrel{\left([\bar{y}]_{n}^{*}\right)_{\sharp}}{\longrightarrow} \mathscr{W}_{n}(M) .
\end{gathered}
$$

On notera que $\varrho_{M}$ est l'homomorphisme composé

$$
\varrho_{M}: \pi_{1}(C, \bar{x}) \stackrel{\xi_{\bar{y}}}{\rightarrow} G^{\text {op }} \rightarrow \operatorname{Aut}_{\mathfrak{o}_{n}} \Gamma\left(\widetilde{E}_{s}^{\prime}, \Phi_{n}^{*}(M)\right)_{\sharp} \stackrel{\left([\bar{y}]_{n}^{*}\right)_{\sharp}}{\sim} \operatorname{Aut}_{\mathfrak{o}_{n}} \mathscr{W}_{n}(M) .
$$

On définit la $\mathfrak{o}_{n}$-représentation de $\pi_{1}(C, \bar{x})$ associée à $M$ par $\left(\mathscr{W}_{n}(M), \varrho_{M}\right)$ que l'on note simplement $\mathscr{W}_{n}(M)$. 
Proposition 8.11. Étant donné un $\overline{\mathscr{B}}_{n}$-module potentiellement libre de type fini $M$, la $\mathfrak{o}_{n}$-représentation $\mathscr{W}_{n}(M)$ de $\pi_{1}(C, \bar{x})$ est indépendante des choix du trait $\left(S^{\prime}, \eta^{\prime}\right)$ fini sur $(S, \eta)$, du $\eta^{\prime}$-revêtement semi-stable, régulier et galoisien $\varphi: X^{\prime} \rightarrow X_{S^{\prime}}$ et du point $\bar{y}$ de $C^{\prime}(\bar{\eta})$ au-dessus de $\bar{x}$ à isomorphisme près.

Preuve. Le $\mathfrak{o}_{n}$-module (8.9.1) sous-jacent à $\mathscr{W}_{n}(M)$ est clairement indépendant des données ci-dessus. Il suffit de démontrer que l'isomorphisme (8.10.4) est indépendant des mêmes données.

Reprenons les notations de 8.9, et soit $\bar{y}$ et $\bar{y}^{\prime}$ deux $\bar{\eta}$-points de $C^{\prime}$ au-dessus de $\bar{x}$. Il existe un élément $h \in G \simeq \operatorname{Aut}\left(C^{\prime} / C\right)$ tel que $h(\bar{y})=\bar{y}^{\prime}$. Avec les notations de 6.6, on a deux homomorphismes

$$
\xi_{\bar{y}}: \pi_{1}(C, \bar{x}) \rightarrow G^{\mathrm{op}} \quad \xi_{\bar{y}^{\prime}}: \pi_{1}(C, \bar{x}) \rightarrow G^{\mathrm{op}}
$$

reliés par la relation (6.6.3):

$$
\xi_{\bar{y}^{\prime}}(\gamma)=h \xi_{\bar{y}}(\gamma) h^{-1} \quad \forall \gamma \in \pi_{1}(C, \bar{x}) .
$$

On déduit par fonctorialité un diagramme commutatif

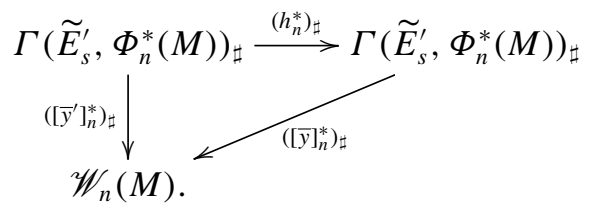

Pour tout $\gamma \in \pi_{1}(C, \bar{x})$, posant $g=\xi_{\bar{y}}(\gamma)$ et $g^{\prime}=\xi_{\bar{y}^{\prime}}(\gamma)$, on en déduit que $g^{\prime}=h g h^{-1}$ dans $G$ et que

$$
\begin{aligned}
& \left(\left[\bar{y}^{\prime}\right]_{n}^{*}\right)_{\sharp} \circ\left(g_{n}^{\prime *}\right)_{\sharp} \circ\left(\left[\bar{y}^{\prime}\right]_{n}^{*}\right)_{\sharp}^{-1} \simeq\left([\bar{y}]_{n}^{*}\right)_{\sharp} \circ\left(h_{n}^{*}\right)_{\sharp} \circ\left(g_{n}^{\prime *}\right)_{\sharp} \circ\left(h_{n}^{*}\right)_{\sharp}^{-1} \circ\left(\left([\bar{y}]_{n}^{*}\right)_{\sharp}\right)^{-1} \\
& \quad=\left([\bar{y}]_{n}^{*}\right)_{\sharp} \circ\left(g_{n}^{*}\right)_{\sharp} \circ\left(\left([\bar{y}]_{n}^{*}\right)_{\sharp}\right)^{-1} .
\end{aligned}
$$

Cela implique que l'automorphisme $\varrho_{M}(\gamma)(8.10 .4)$ est indépendant du choix du point $\bar{y}$ au-dessus de $\bar{x}$.

Soit $\left(S^{\prime \prime}, \eta^{\prime \prime}\right)$ un trait fini sur $(S, \eta)$ et $\phi: X^{\prime \prime} \rightarrow X_{S^{\prime \prime}}$ un $\eta^{\prime \prime}$-revêtement semistable, régulier et galoisien trivialisant $M$. D'après 5.11(i), on peut supposer que $S^{\prime \prime}$ domine $S^{\prime}$ et qu'il existe un morphisme équivariant de $\phi$ dans $\varphi \times_{S^{\prime}} S^{\prime \prime}$ (cf. 5.4). Notons-le $\psi: X^{\prime \prime} \rightarrow X^{\prime} \times_{S^{\prime}} S^{\prime \prime}$ et soit $u: \operatorname{Aut}\left(X_{\eta^{\prime \prime}}^{\prime \prime} / X_{\eta^{\prime \prime}}\right) \rightarrow \operatorname{Aut}\left(X_{\eta^{\prime \prime}}^{\prime} / X_{\eta^{\prime \prime}}\right)$ l'homomorphisme induit par $\psi_{\eta^{\prime \prime}}$. Alors, le morphisme équivariant $\psi$ induit un morphisme $u$-équivariant de topos annelés de Faltings :

$$
\Psi_{n}:\left(\widetilde{E}_{s}^{\prime \prime}, \overline{\mathscr{B}}_{n}^{\prime \prime}\right) \rightarrow\left(\widetilde{E}_{s}^{\prime}, \overline{\mathscr{B}}_{n}^{\prime}\right),
$$


où la source désigne le topos annelé de Faltings associé au $S^{\prime \prime}$-schéma $X^{\prime \prime}$, qui est muni d'une action de $\operatorname{Aut}\left(X_{\eta^{\prime \prime}}^{\prime \prime} / X_{\eta^{\prime \prime}}\right)$ (8.4). On en déduit, pour tout $g \in$ $\operatorname{Aut}\left(X_{\eta^{\prime \prime}}^{\prime \prime} / X_{\eta^{\prime \prime}}\right)$, un diagramme commutatif

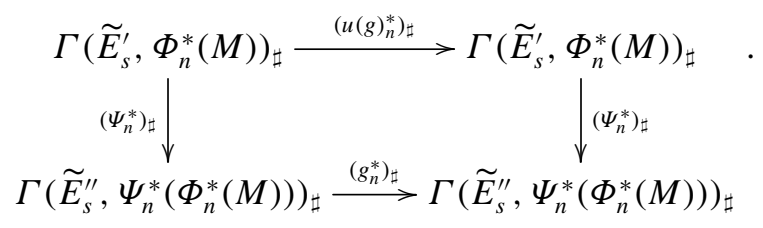

La proposition s'ensuit compte tenu de (8.10.4).

8.12. Soit $f: M \rightarrow M^{\prime}$ un morphisme de $\overline{\mathscr{B}}_{n}$-modules potentiellement libres de type fini. D'après 5.11(i), il existe un trait $\left(S^{\prime}, \eta^{\prime}\right)$ fini sur $(S, \eta)$ et un $\eta^{\prime}$ revêtement semi-stable, régulier et galoisien $\varphi: X^{\prime} \rightarrow X_{S^{\prime}}$ tels que, reprenant les notations de $8.3, \Phi_{n}^{*}(M)$ et $\Phi_{n}^{*}\left(M^{\prime}\right)$ soient libres de type fini. L'automorphisme (8.10.4) est fonctoriel en $M$. Par 8.11, on peut associer à $f: M \rightarrow M^{\prime}$ un morphisme de $\mathfrak{o}_{n}$-représentations de $\pi_{1}(C, \bar{x})$

$$
\mathscr{W}_{n}(f): \mathscr{W}_{n}(M) \rightarrow \mathscr{W}_{n}\left(M^{\prime}\right) .
$$

La correspondance $M \mapsto \mathscr{W}_{n}(M)$ définit ainsi un foncteur $\mathfrak{o}_{n}$-linéaire

$$
\mathscr{W}_{n}: \operatorname{Mod}^{\mathrm{pltf}}\left(\overline{\mathscr{B}}_{n}\right) \rightarrow \operatorname{Rep}_{\mathfrak{o}_{n}}^{\alpha \mathrm{ptf}}\left(\pi_{1}(C, \bar{x})\right) .
$$

LEMME 8.13. Soit $M=\left(M_{n}\right)_{n \geqslant 1}$ un $\breve{\mathscr{B}}$-module potentiellement libre de type fini. Pour tout entier $n \geqslant 1$, le morphisme canonique $M_{n+1} \rightarrow M_{n}$ induit un $\alpha$-isomorphisme fonctoriel de $\mathfrak{o}_{n}$-représentations (3.22)

$$
\mathscr{W}_{n+1}\left(M_{n+1}\right) \otimes_{\mathfrak{o}_{n+1}} \mathfrak{o}_{n} \rightarrow \mathscr{W}_{n}\left(M_{n}\right)
$$

Preuve. Soit $n$ un entier $\geqslant 1$. D'après 5.11(i), il existe un trait $\left(S^{\prime}, \eta^{\prime}\right)$ fini sur $(S, \eta)$ et un $\eta^{\prime}$-revêtement semi-stable, régulier et galoisien $\varphi: X^{\prime} \rightarrow X_{S^{\prime}}$ trivialisant $M_{n+1}$. On a un isomorphisme de $\mathfrak{o}_{n}$-modules

$$
\Gamma\left(s_{\text {ét }},[\bar{x}]_{n+1}^{*}\left(M_{n+1}\right)\right) \otimes_{\mathfrak{o}_{n+1}} \mathfrak{o}_{n} \stackrel{\sim}{\rightarrow} \Gamma\left(s_{\text {ét }},[\bar{x}]_{n}^{*}\left(M_{n}\right)\right) .
$$

En vertu de (3.1.2), celui-ci induit un $\alpha$-isomorphisme canonique de $\mathfrak{o}_{n}$-modules

$$
\mathscr{W}_{n+1}\left(M_{n+1}\right) \otimes_{\mathfrak{o}_{n+1}} \mathfrak{o}_{n} \rightarrow \mathscr{W}_{n}\left(M_{n}\right)
$$

D'après 8.11 et la fonctorialité de (8.10.4), ce dernier est $\pi_{1}(C, \bar{x})$-équivariant. On vérifie aussitôt que (8.13.1) est fonctoriel en $M$ (cf. 8.12). 
8.14. En vertu de (3.23.1) et 8.13 , les foncteurs $\mathscr{W}_{n}$ induisent un foncteur o-linéaire

$$
\begin{aligned}
\mathscr{W}: \operatorname{Mod}^{\mathrm{plff}}(\tilde{\mathscr{B}}) & \rightarrow \operatorname{Rep}_{\mathfrak{o}}^{\alpha \mathrm{ptf}}\left(\pi_{1}(C, \bar{x})\right) \\
M=\left(M_{n}\right)_{n \geqslant 1} & \mapsto \lim _{\leftarrow} \otimes_{\mathfrak{o}} \mathscr{W}_{n}\left(M_{n}\right) .
\end{aligned}
$$

On le note aussi $\mathscr{W}_{X}$ pour signifier que la construction dépend de $X$. D'après 3.27, le foncteur $\mathscr{W}$ s'étend en un foncteur $\mathfrak{C}$-linéaire:

$$
\mathscr{W}_{\mathbb{Q}}: \operatorname{Mod}_{\mathbb{Q}}^{\text {pltf }}(\breve{\mathscr{B}}) \rightarrow \operatorname{Rep}_{\mathfrak{C}}^{\text {cont }}\left(\pi_{1}(C, \bar{x})\right)
$$

Proposition 8.15. Soit $\left(S^{\prime}, \eta^{\prime}\right)$ un trait fini $\operatorname{sur}(S, \eta)$ et $\varphi: X^{\prime} \rightarrow X_{S^{\prime}}$ un $\eta^{\prime}$-revêtement semi-stable et régulier. Reprenant les notations de 8.3 pour $\varphi$, l'image réciproque par $\breve{\Phi}(8.3 .15)$ d'un $\breve{\mathscr{B}}$-module potentiellement libre de type fini est un $\breve{\mathscr{B}}$-module potentiellement libre de type fini. De plus, on a un diagramme commutatif

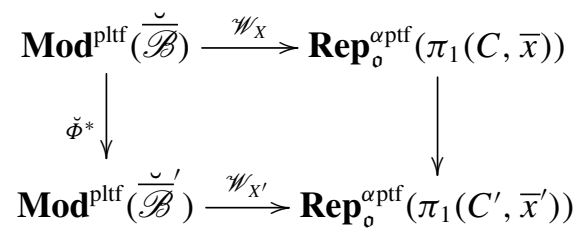

où $C^{\prime}=X_{\bar{\eta}}^{\prime}, \bar{x}^{\prime}$ est un $\eta$-point de $C^{\prime}$ au-dessus de $\bar{x}$ et la flèche verticale à droite est le foncteur induit par l'homomorphisme canonique $\pi_{1}\left(C^{\prime}, \bar{x}^{\prime}\right) \rightarrow \pi_{1}(C, \bar{x})$.

Preuve. Soit $n$ un entier $\geqslant 1$ et $M_{n}$ un $\overline{\mathscr{B}}_{n}$-module potentiellement libre de type fini. D'après 5.11(i), quitte à remplacer $S^{\prime}$ par une extension finie, il existe un $\eta^{\prime}$-revêtement semi-stable, régulier et galoisien $\psi: X^{\prime \prime} \rightarrow X_{S^{\prime}}$ dominant $\varphi: X^{\prime} \rightarrow X_{S^{\prime}}$ tel que $\Psi_{n}^{*}\left(M_{n}\right)$ soit un $\widetilde{\mathscr{B}}_{n}^{\prime \prime}$-module libre de type fini, où $\left(\widetilde{E}_{s}^{\prime \prime}\right.$, $\left.\overline{\mathscr{B}}_{n}^{\prime \prime}\right)$ désigne le topos annelé de Faltings associé à la $S^{\prime}$-courbe semi-stable $X^{\prime \prime}, \Psi_{n}$ le morphisme de topos annelés induit par $\psi$ (8.3.12). Le $\overline{\mathscr{B}}_{n}^{\prime}$-module $\Phi_{n}^{*}\left(M_{n}\right)$ est donc potentiellement libre de type fini. On en déduit la première proposition.

Notons $\phi: X^{\prime \prime} \rightarrow X^{\prime}$ le morphisme induit par $\psi, \mathscr{W}_{X, n}\left(M_{n}\right)$ et $\mathscr{W}_{X^{\prime}, n}\left(\Phi_{n}^{*}\left(M_{n}\right)\right)$ les $\mathfrak{o}_{n}$-représentations associées à $M_{n}$ et $\Phi_{n}^{*}\left(M_{n}\right)$, respectivement. On notera que 
$\phi$ est un $\eta^{\prime}$-revêtement semi-stable, régulier et galoisien de $X^{\prime}$. D'après (8.10.1), on a un isomorphisme de $\mathfrak{o}_{n}$-modules

$$
\mathscr{W}_{X, n}\left(M_{n}\right) \stackrel{\sim}{\rightarrow} \mathscr{W}_{X^{\prime}, n}\left(\Phi_{n}^{*}\left(M_{n}\right)\right) .
$$

En vertu de (8.10.4) et 8.11 , celui-là est $\pi_{1}\left(C^{\prime}, \bar{x}^{\prime}\right)$-équivariant. On vérifie aussitôt que l'isomorphisme (8.15.2) est fonctoriel en $M_{n}$ (cf. 8.12). La commutativité du diagramme (8.15.1) s'ensuit.

Proposition 8.16. Soit $n$ un entier $\geqslant 1$ et $V$ un objet de $\operatorname{Rep}_{\mathfrak{o}_{n}}^{\text {lff }}\left(\pi_{1}(C, \bar{x})\right)$. Alors, on a un $\alpha$-isomorphisme canonique et fonctoriel de $\mathfrak{o}_{n}$-représentations

$$
V \rightarrow \mathscr{W}_{n}\left(\beta_{n}^{*}(V)\right)
$$

où $\beta_{n}^{*}$ est le foncteur (8.6.1) (cf. 8.7).

Preuve. Soit $\phi: C^{\prime} \rightarrow C$ un revêtement étale, connexe et galoisien tel que l'action de $\pi_{1}(C, \bar{x})$ sur $V$ se factorise à travers $\operatorname{Aut}\left(C^{\prime} / C\right)^{\text {op }}$. D'après 5.11(i), il existe un trait $\left(S^{\prime}, \eta^{\prime}\right)$ fini sur $(S, \eta)$ et un $\eta^{\prime}$-revêtement semi-stable, régulier et galoisien $\varphi: X^{\prime} \rightarrow X_{S^{\prime}}$ de fibre géométrique générique $\varphi_{\bar{\eta}}=\phi: C^{\prime} \rightarrow C$. Reprenons les notations de 8.3 et 8.4 ; pour $\varphi: X^{\prime} \rightarrow X_{S^{\prime}}$, tout $g \in \operatorname{Aut}\left(C^{\prime} / C\right)$ induit un diagramme commutatif de topos annelés (cf. (8.3.14))

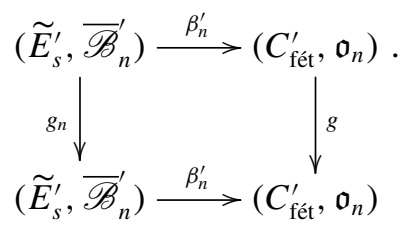

On note $\mathbb{L}=\mu_{\bar{x}}(V)$ le $\mathfrak{o}_{n}$-module localement libre de type fini de $C_{\text {fét }}$ associé à $V$ (3.29.2). Choisissons un $\bar{\eta}$-point $\bar{y}$ de $C^{\prime}$ au-dessus $\bar{x}$. On désigne par

$$
\tau(V): V \stackrel{\sim}{\rightarrow} \mathscr{W}_{n}\left(\beta_{n}^{*}(\mathbb{L})\right)
$$

le $\alpha$-isomorphisme de $\mathfrak{o}_{n}$-modules défini par le composé

$$
\begin{aligned}
V & \stackrel{\sim}{\rightarrow} \Gamma\left(C_{\text {fét }}^{\prime}, \phi^{*}(\mathbb{L})\right) \rightarrow \Gamma\left(\widetilde{E}_{s}^{\prime}, \beta_{n}^{* *}\left(\phi^{*}(\mathbb{L})\right)\right) \rightarrow \Gamma\left(\widetilde{E}_{s}^{\prime}, \beta_{n}^{\prime *}\left(\phi^{*}(\mathbb{L})\right)\right)_{\sharp} \\
& \stackrel{\sim}{\rightarrow} \Gamma\left(\widetilde{E}_{s}^{\prime}, \Phi_{n}^{*}\left(\beta_{n}^{*}(\mathbb{L})\right)\right)_{\sharp} \stackrel{\left([\bar{y}]_{n}^{*}\right)_{\sharp}}{\longrightarrow} \mathscr{W}_{n}\left(\beta_{n}^{*}(\mathbb{L})\right)
\end{aligned}
$$

où la première flèche est un isomorphisme puisque $\phi^{*}(\mathbb{L})$ est constant et $C^{\prime}$ est connexe, la deuxième flèche est le $\alpha$-isomorphisme induit par $\beta_{n}^{* *}$ (7.20.1), la troisième flèche est un $\alpha$-isomorphisme en vertu de (3.1.2), la quatrième flèche 
provient de (8.3.14) et la dernière flèche est l'isomorphisme (8.2.4). D'après (8.16.2), pour tout $g \in \operatorname{Aut}\left(C^{\prime} / C\right)$, le morphisme (8.16.4) s'insère dans un diagramme commutatif :

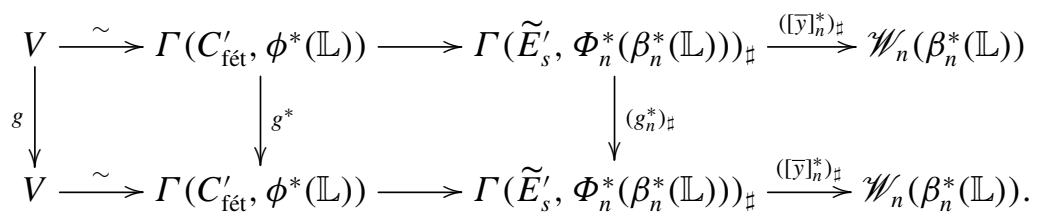

(8.16.5)

Celui-ci implique que le $\alpha$-isomorphisme $\tau(V)$ (8.16.3) est compatible aux actions de $\pi_{1}(C, \bar{x})$ en vertu de (8.10.4). Par suite, on obtient un $\alpha$-isomorphisme $\operatorname{de} \operatorname{Rep}_{\mathfrak{o}_{n}}^{\alpha \mathrm{ptf}}\left(\pi_{1}(C, \bar{x})\right)$

$$
\tau(V): V \rightarrow \mathscr{W}_{n}\left(\beta_{n}^{*}(V)\right)
$$

Soit $f: V^{\prime} \rightarrow V$ un morphisme de $\operatorname{Rep}_{\mathfrak{o}_{n}}^{\text {lff }}\left(\pi_{1}(C, \bar{x})\right)$. Choisissons un revêtement étale et galoisien $\phi: C^{\prime} \rightarrow C$ trivialisant les représentations $V$ et $V^{\prime}$, un trait $\left(S^{\prime}, \eta^{\prime}\right)$ fini sur $(S, \eta)$ et un $\eta^{\prime}$-revêtement semi-stable, régulier et galoisien $\varphi: X^{\prime} \rightarrow X_{S^{\prime}}$ de fibre générique géométrique $\phi: C^{\prime} \rightarrow C$. Le diagramme commutatif (8.16.5) étant fonctoriel en $V$, on en déduit un diagramme commutatif

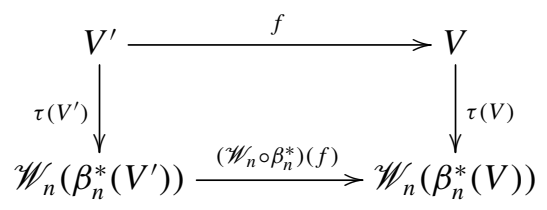

d'où la fonctorialité du $\alpha$-isomorphisme (8.16.1).

Corollaire 8.17. (i) Soit $V$ un objet de $\operatorname{Rep}_{\mathfrak{o}}^{\mathrm{ltf}}\left(\pi_{1}(C, \bar{x})\right)$. On a un isomorphisme canonique fonctoriel

$$
\mathfrak{m} \otimes_{\mathfrak{o}} V \stackrel{\sim}{\rightarrow} \mathscr{W}\left(\breve{\beta}^{*}(V)\right)
$$

(ii) Le foncteur composé $\mathscr{W}_{\mathbb{Q}} \circ \breve{\beta}_{\mathbb{Q}}^{*}(8.6 .3)$ est isomorphe au foncteur identique.

Preuve. Pour tout entier $n \geqslant 1$, on pose $V_{n}=V / p^{n} V$. D'après 8.16 , on a un $\alpha$-isomorphisme canonique et fonctoriel $\left(V_{n}\right)_{n \geqslant 1} \rightarrow\left(\mathscr{W}_{n}\left(\beta_{n}^{*}\left(V_{n}\right)\right)\right)_{n \geqslant 1}$ 
de $\operatorname{Rep}_{\breve{o}}^{\alpha p t f}\left(\pi_{1}(C, \bar{x})\right)$ (3.23). L'assertion (i) résulte alors de 3.24(i) et 3.25(ii). L'assertion (ii) résulte de (i) et de l'isomorphisme canonique $\left(\mathfrak{m} \otimes_{\mathfrak{o}} V\right)\left[\frac{1}{p}\right] \rightarrow$ $V\left[\frac{1}{p}\right]$.

8.18. Posons $\check{\bar{X}}=X \times_{S} \stackrel{\check{S}}{S}$ (2.1). Pour tout entier $n \geqslant 1$ et tout $\mathscr{O}_{\overline{\mathrm{X}}}$-module $\mathcal{F}$, on considère $\mathcal{F}_{n}=\mathcal{F} \otimes_{\mathscr{O}_{S}} \mathscr{O}_{S_{n}}$ comme un faisceau de $X_{s \text {,ét. }}$ On pose $\breve{\mathcal{F}}=\left(\mathcal{F}_{n}\right)_{n \geqslant 1}$ que l'on considère aussi comme un faisceau de $X_{s \text {,ét }}^{\mathbb{N}^{\circ}}$. D'après [4, III.7.18], on a un isomorphisme $\breve{\sigma}^{*}(\breve{\mathcal{F}}) \simeq\left(\sigma_{n}^{*}\left(\mathcal{F}_{n}\right)\right)_{n \geqslant 1}$, où $\sigma_{n}$ et $\breve{\sigma}$ sont des morphismes de topos annelés (7.11.4) et (7.12.1).

Proposition 8.19. Soit $n$ un entier $\geqslant 1$ et $\mathcal{F}$ un fibré vectoriel de Deninger-

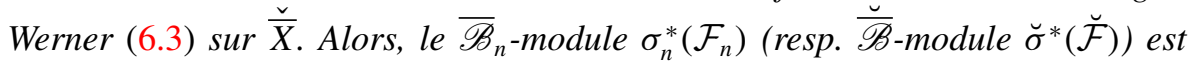
potentiellement libre de type fini.

Preuve. D'après 5.11(i), quitte à remplacer $S$ par une extension finie, il existe un $\eta$-revêtement semi-stable $\varphi: X^{\prime} \rightarrow X$ tel que $\bar{\varphi}_{n}$ trivialise $\mathcal{F}_{n}$. Reprenons les notations de 8.3 pour $\varphi$. D'après (8.3.13), on a un isomorphisme

$$
\Phi_{n}^{*}\left(\sigma_{n}^{*}\left(\mathcal{F}_{n}\right)\right) \simeq \sigma_{n}^{\prime *}\left(\bar{\varphi}_{n}^{*}\left(\mathcal{F}_{n}\right)\right)
$$

Le $\overline{\mathscr{B}}_{n}$-module $\sigma_{n}^{*}\left(\mathcal{F}_{n}\right)$ est donc potentiellement libre de type fini. Comme $\breve{\mathcal{F}}$ est adique, on en déduit que le $\breve{\mathscr{B}}$-module $\breve{\sigma}^{*}(\breve{\mathcal{F}})$ est potentiellement libre de type fini.

Proposition 8.20. Soit $n$ un entier $\geqslant 1$ et $\mathcal{F}$ un fibré vectoriel de DeningerWerner sur $\check{\bar{X}}$. On a un $\alpha$-isomorphisme canonique et fonctoriel

$$
\mathbb{V}_{n}(\mathcal{F}) \rightarrow \mathscr{W}_{n}\left(\sigma_{n}^{*}\left(\mathcal{F}_{n}\right)\right)
$$

où $\mathbb{V}_{n}$ est le foncteur de Deninger-Werner (6.9.2).

Preuve. D'après 5.11(i), quitte à remplacer $S$ par une extension finie, il existe un $\eta$-revêtement semi-stable, régulier et galoisien $\varphi: X^{\prime} \rightarrow X$ tel que $\bar{\varphi}_{n}$ trivialise $\mathcal{F}_{n}$. Reprenant les notations de 8.1 et 8.3 , on a un diagramme commutatif de topos 
annelés :

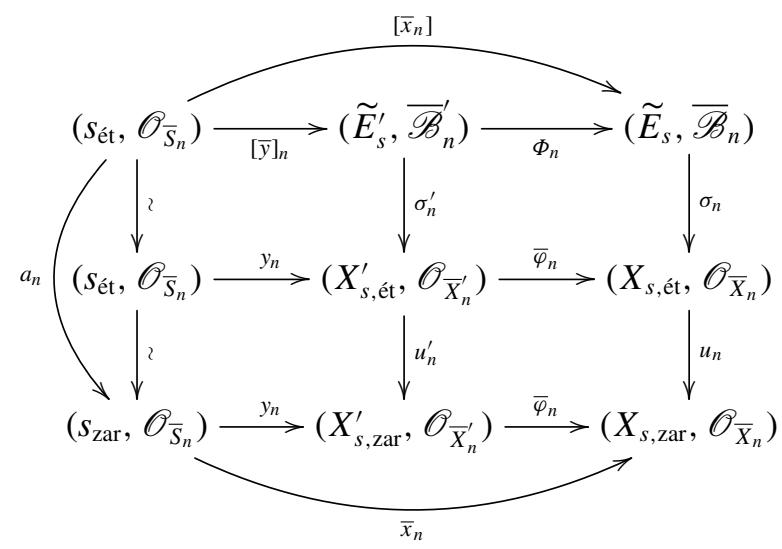

où $u_{n}$ et $u_{n}^{\prime}$ sont les morphismes de topos annelés canoniques. Rappelons $((6.6 .1)$ et $(8.9 .1))$ que les $\mathfrak{o}_{n}$-modules sous-jacents aux représentations $\mathbb{V}_{n}(\mathcal{F})$ et $\mathscr{W}_{n}\left(\sigma_{n}^{*}\left(\mathcal{F}_{n}\right)\right)$ sont définis par

$$
\mathbb{V}_{n}(\mathcal{F})=\Gamma\left(s_{\text {zar }}, \bar{x}_{n}^{*}\left(\mathcal{F}_{n}\right)\right) \quad \text { et } \quad \mathscr{W}_{n}\left(\sigma_{n}^{*}\left(\mathcal{F}_{n}\right)\right)=\Gamma\left(s_{\text {ét }},\left[x_{n}\right]^{*}\left(\sigma_{n}^{*}\left(\mathcal{F}_{n}\right)\right)\right)_{\sharp} .
$$

Comme on a $\left[\bar{x}_{n}\right]^{*}\left(\sigma_{n}^{*}\left(\mathcal{F}_{n}\right)\right) \simeq a_{n}^{*}\left(\bar{x}_{n}^{*}\left(\mathcal{F}_{n}\right)\right)$ (8.20.2), l'équivalence de topos annelés $a_{n}$ et le foncteur $(-)_{\sharp}(3.1 .1)$ induisent un $\alpha$-isomorphisme de $\mathfrak{o}_{n}$-modules

$$
a_{n}^{*}: \mathbb{V}_{n}(\mathcal{F}) \rightarrow \mathscr{W}_{n}\left(\sigma_{n}^{*}\left(\mathcal{F}_{n}\right)\right) .
$$

Posons $C^{\prime}=X_{\bar{\eta}}^{\prime}$ et $G=\operatorname{Aut}\left(C^{\prime} / C\right)$. Reprenant les notations de 8.4, les carrés commutatifs de droite de (8.20.2) sont compatibles aux actions de $G \operatorname{sur}\left(\widetilde{E}_{s}^{\prime}, \overline{\mathscr{B}}_{n}^{\prime}\right)$, $\left(X_{s, \text { ét }}^{\prime}, \mathscr{O}_{\breve{X}_{n}^{\prime}}\right)$ et $\left(X_{s, \text { zar }}^{\prime}, \mathscr{O}_{\bar{X}_{n}^{\prime}}\right)$. On en déduit par le foncteur $(-)_{\sharp}$, pour tout $g \in G$, un diagramme commutatif :

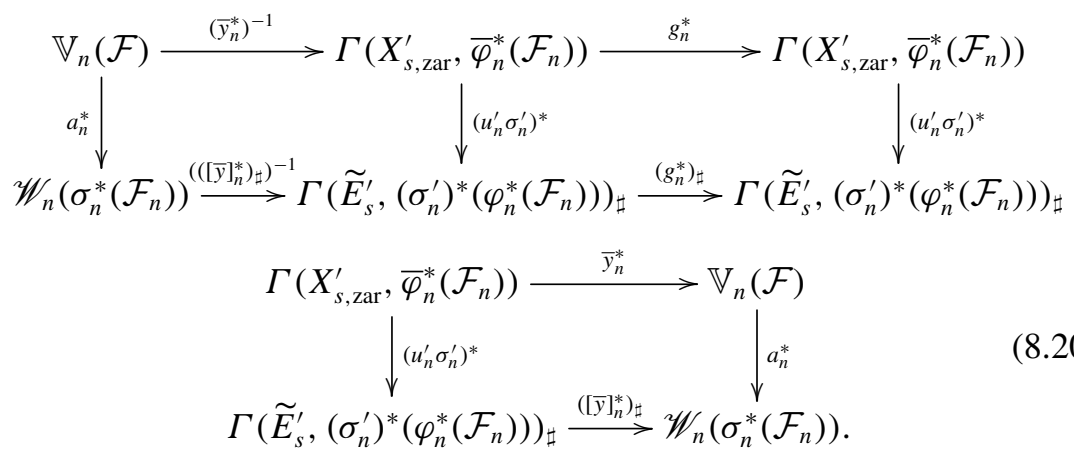


Celui-ci implique que le $\alpha$-isomorphisme (8.20.3) est compatible aux actions de $\pi_{1}(C, \bar{x})$ en vertu de (6.7.3) et (8.10.4). On en déduit un $\alpha$-isomorphisme de $\mathfrak{o}_{n^{-}}$ représentations de $\pi_{1}(C, \bar{x})$

$$
\tau(\mathcal{F}): \mathbb{V}_{n}(\mathcal{F}) \rightarrow \mathscr{W}_{n}\left(\sigma_{n}^{*}(\mathcal{F})\right) .
$$

Soit $f: \mathcal{F}^{\prime} \rightarrow \mathcal{F}$ un morphisme de $\mathfrak{V}_{\bar{X}}^{\text {DW }}$. D'après 5.11(i), quitte à remplacer $S$ par une extension finie, il existe un $\eta$-revêtement semi-stable et régulier $\varphi: X^{\prime} \rightarrow$ $X$ tels que $\bar{\varphi}_{n}$ trivialise $\mathcal{F}_{n}$ et $\mathcal{F}_{n}^{\prime}$. Le diagramme commutatif (8.20.4) est fonctoriel en $\mathcal{F}$. On en déduit par 6.8 et 8.11 un diagramme commutatif

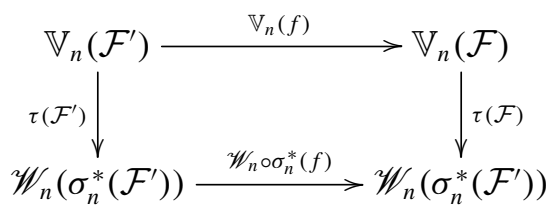

d'où la fonctorialité du $\alpha$-isomorphisme (8.20.1).

COROllaire 8.21. Soit $\mathcal{F}$ un fibré vectoriel de Deninger-Werner sur $\check{\bar{X}}$. On a un isomorphisme canonique et fonctoriel de o-représentations continues de $\pi_{1}(C, \bar{x})$

$$
\mathfrak{m} \otimes_{\mathfrak{o}} \mathbb{V}(\mathcal{F}) \stackrel{\sim}{\rightarrow} \mathscr{W}\left(\breve{\sigma}^{*}(\breve{\mathcal{F}})\right)
$$

où $\mathbb{V}$ est le foncteur de Deninger-Werner (6.10.1).

Preuve. D'après 8.20, on a un $\alpha$-isomorphisme canonique et fonctoriel $\left(\mathbb{V}_{n}(\mathcal{F})\right)_{n \geqslant 1} \rightarrow\left(\mathscr{W}_{n}\left(\sigma_{n}^{*}\left(\mathcal{F}_{n}\right)\right)\right)_{n \geqslant 1}$ de $\operatorname{Rep}_{\mathfrak{o}}^{\alpha \text { ptf }}\left(\pi_{1}(C, \bar{x})\right)$ (3.23). L'assertion résulte alors de 3.24(i) et 3.25(ii).

\section{Un énoncé de descente pour les fibrés vectoriels de Deninger-Werner}

9.1. Soit $\mathcal{T}$ un topos et $G$ un groupe fini. Une action de $G$ sur $\mathcal{T}$ est la donnée pour tout $g \in G$ d'un morphisme de topos $g: \mathcal{T} \rightarrow \mathcal{T}$ tels que (id)* ${ }^{*} \mathrm{id}_{\mathcal{T}}$ et que pour tous $g, h \in G$, on ait un isomorphisme

$$
c_{g, h}: g^{*} h^{*} \stackrel{\sim}{\rightarrow}(h g)^{*}
$$

vérifiant des relations de cocycle pour la composition [23, VI 7.4]. Supposons que $\mathcal{T}$ soit muni d'une telle action de $G$. Un objet $G$-équivariant de $\mathcal{T}$ est la donnée d'un faisceau $\mathscr{F}$ de $\mathcal{T}$, et pour tout $g \in G$, d'un isomorphisme

$$
\tau_{g}^{\mathscr{F}}: \mathscr{F} \stackrel{\sim}{\rightarrow} g^{*}(\mathscr{F})
$$


tels que $\tau_{\text {id }}^{\mathscr{F}}=\mathrm{id} \mathscr{F}$ et que pour tous $g, h \in G$, on ait

$$
\tau_{g h}^{\mathscr{F}}=c_{h, g} \circ h^{*}\left(\tau_{g}^{\mathscr{F}}\right) \circ \tau_{h}^{\mathscr{F}} .
$$

Une telle donnée est appelée une action de $G$ (à droite) sur $\mathscr{F}$. Étant donné deux objets $G$-équivariants $\mathscr{F}_{1}$ et $\mathscr{F}_{2}$, un morphisme $f: \mathscr{F}_{1} \rightarrow \mathscr{F}_{2}$ de $\mathcal{T}$ est dit $G$ équivariant si, pour tout $g \in G$, le diagramme

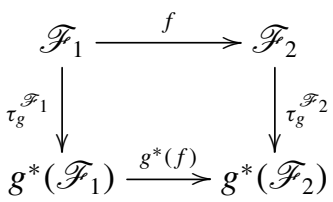

est commutatif. On désigne par $\mathcal{T}_{G}$ la catégorie des objets $G$-équivariants de $\mathcal{T}$.

Soit $A$ un anneau de $\mathcal{T}$ muni d'une action de $G$ compatible avec sa structure d'anneau, i.e. tel que les isomorphismes $\tau_{g}^{A}(9.1 .1)$ soient des isomorphismes d'anneaux. Un A-module $G$-équivariant est la donnée d'un $A$-module $M$ de $\mathcal{T}$ et d'une action de $G$ sur $M$ compatible avec sa structure de $A$-module.

9.2. Dans la suite de cette section, on se donne une $S$-courbe semi-stable $X$ et un revêtement étale et galoisien $C^{\prime}$ de $C=X_{\bar{\eta}}$. On pose $G=\operatorname{Aut}\left(C^{\prime} / C\right)$ et on note $\psi: C^{\prime} \rightarrow C$ le morphisme canonique. D’après 5.10, le schéma $\bar{X}$ est normal. On reprend les notations de la Section 7 pour $X$. On note $E^{\sharp}$ le site défini par la catégorie $E_{/\left(C^{\prime} \rightarrow X\right)}(7.1)$ munie de la topologie induite par le foncteur canonique

$$
\gamma: E_{/\left(C^{\prime} \rightarrow X\right)} \rightarrow E
$$

Le site $E^{\sharp}$ est alors canoniquement équivalent au site de Faltings associé au morphisme canonique $C^{\prime} \rightarrow X$ (cf. [4, VI.10.1 et VI.10.14]). On désigne par $\widetilde{E}^{\sharp}$ le topos localisé de $\widetilde{E}$ en $\beta^{*}\left(C^{\prime}\right)=\left(C^{\prime} \rightarrow X\right)^{a}$ qui est canoniquement équivalent au topos des faisceaux d'ensembles sur le site $E^{\sharp}$ [4, VI.10.14] et par

$$
\Phi: \widetilde{E}^{\sharp} \rightarrow \widetilde{E}
$$

le morphisme de localisation. En vertu de [6, III 2.5], celui-là s'identifie au morphisme de topos induit par fonctorialité du topos de Faltings [4, VI.10.12]. On désigne par $\widetilde{E}_{s}^{\sharp}$ le sous-topos fermé de $\widetilde{E}^{\sharp}$ complémentaire de l'ouvert $\Phi^{*}\left(\sigma^{*}\left(X_{\eta}\right)\right)$ de $\widetilde{E}^{\sharp}$ et par

$$
\delta^{\sharp}: \widetilde{E}_{s}^{\sharp} \rightarrow \widetilde{E}^{\sharp}
$$

le prolongement canonique. En vertu de [6, IV 9.4.3], il existe un morphisme de topos canonique

$$
\Phi_{s}: \widetilde{E}_{s}^{\sharp} \rightarrow \widetilde{E}_{s}
$$


On pose $\overline{\mathscr{B}}^{\sharp}=\Phi^{*}(\overline{\mathscr{B}})(7.8)$ et, pour tout entier $n \geqslant 1, \overline{\mathscr{B}}_{n}^{\sharp}=\overline{\mathscr{B}}^{\sharp} / p^{n} \overline{\mathscr{B}}^{\sharp}$ qui est isomorphe à l'anneau $\Phi^{*}\left(\overline{\mathscr{B}}_{n}\right)$. Comme $\overline{\mathscr{B}}_{n}$ est un objet de $\widetilde{E}_{s}(7.10)$, on en déduit que l'anneau $\overline{\mathscr{B}}_{n}^{\sharp}$ appartient à $\widetilde{E}_{s}^{\sharp}$. Le morphisme de topos (9.2.4) est donc sous-jacent à un morphisme de topos annelés que l'on note

$$
\Phi_{n}:\left(\widetilde{E}_{s}^{\sharp}, \overline{\mathscr{B}}_{n}^{\sharp}\right) \rightarrow\left(\widetilde{E}_{s}, \overline{\mathscr{B}}_{n}\right) .
$$

9.3. Par fonctorialité du topos de Faltings [4, VI.10.12], l'action de $G$ sur $C^{\prime}$ induit une action de $G$ sur le topos $\widetilde{E}^{\sharp}$ (9.1). En particulier, tout $g \in G$ induit un morphisme $g: \widetilde{E}^{\sharp} \rightarrow \widetilde{E}^{\sharp}$ tel que

$$
\Phi \stackrel{\sim}{\rightarrow} \Phi \circ g
$$

On en déduit que $g^{*}\left(\Phi^{*}\left(\sigma^{*}\left(X_{\eta}\right)\right)\right) \simeq \Phi^{*}\left(\sigma^{*}\left(X_{\eta}\right)\right)$. L'action de $G$ sur $\widetilde{E}^{\sharp}$ induit alors une action de $G$ sur $\widetilde{E}_{s}^{\sharp}$. Pour tout $g \in G$, on a donc un morphisme $g_{s}$ : $\widetilde{E}_{s}^{\sharp} \rightarrow \widetilde{E}_{s}^{\sharp}$ tel que

$$
\Phi_{s} \stackrel{\sim}{\rightarrow} \Phi_{s} \circ g_{s} .
$$

On désigne par $\widetilde{E}_{G}^{\sharp}$ la catégorie des objets $G$-équivariants de $\widetilde{E}^{\sharp}$ (9.1). D'après (9.3.1), l'image inverse de $\Phi$ induit un foncteur

$$
\Phi^{*}: \widetilde{E} \rightarrow \widetilde{E}_{G}^{\sharp}
$$

En particulier, l'anneau $\overline{\mathscr{B}}_{n}^{\sharp}$ est muni d'une action de $G$ compatible avec sa structure d'anneau. Pour tout entier $n \geqslant 1$ et tout $g \in G$, le morphisme de topos $g_{s}$ est sous-jacent à un morphisme de topos annelés

$$
g_{n}:\left(\widetilde{E}_{s}^{\sharp}, \overline{\mathscr{B}}_{n}^{\sharp}\right) \rightarrow\left(\widetilde{E}_{s}^{\sharp}, \overline{\mathscr{B}}_{n}^{\sharp}\right)
$$

tel que l'on ait un isomorphisme canonique

$$
\Phi_{n} \stackrel{\sim}{\rightarrow} \Phi_{n} \circ g_{n} .
$$

L'image inverse de $\Phi_{n}$ induit alors un foncteur

$$
\Phi_{n}^{*}: \operatorname{Mod}\left(\overline{\mathscr{B}}_{n}\right) \rightarrow \operatorname{Mod}_{G}\left(\overline{\mathscr{B}}_{n}^{\sharp}\right)
$$

où $\operatorname{Mod}_{G}\left(\overline{\mathscr{B}}_{n}^{\sharp}\right)$ désigne la sous-catégorie pleine de $\widetilde{E}_{G}^{\sharp}$ formée des $\overline{\mathscr{B}}_{n}^{\sharp}$-modules $G$-équivariants de $\widetilde{E}_{s}^{\sharp}(9.1)$.

Proposition 9.4. Le foncteur $\Phi^{*}: \widetilde{E} \rightarrow \widetilde{E}_{G}^{\sharp}$ (9.3.3) est une équivalence de catégories. 
Preuve. Considérons

$$
\mathbf{F} \rightarrow E
$$

le topos fibré associé au site $E$ (cf. [22, II 3.4.1]) : la catégorie fibre de $\mathbf{F}$ au-dessus de $(V \rightarrow U) \in \mathbf{O b}(E)$ est le topos localisé $\widetilde{E}_{/(V \rightarrow U)^{a}}$ de $\widetilde{E}$ en $(V \rightarrow U)^{a}$ et, pour tout morphisme $h:\left(V^{\prime} \rightarrow U^{\prime}\right) \rightarrow(V \rightarrow U)$ de $E$, le foncteur image inverse $h^{*}: \widetilde{E}_{/(V \rightarrow U)^{a}} \rightarrow \widetilde{E}_{/\left(V^{\prime} \rightarrow U^{\prime}\right)^{a}}$ est le foncteur image inverse par le morphisme de localisation par rapport à $h$. On rappelle que $\mathbf{F}$ est un champ au-dessus de $E$ (cf. [22, II 3.4.4]).

Le morphisme $\varpi=(\psi, \mathrm{id}):\left(C^{\prime} \rightarrow X\right) \rightarrow(C \rightarrow X)$ forme un recouvrement de $(C \rightarrow X)$ dans $E$ (cf. 7.1(v)) que l'on note (abusivement) encore $\varpi$. La catégorie $\widetilde{E}=\mathbf{F}(C \rightarrow X)$ est donc équivalente à la catégorie $\mathbf{F}(\varpi)$ des données de descente relativement au recouvrement $\varpi$.

Comme le morphisme $\psi: C^{\prime} \rightarrow C$ est un torseur sous $G$ de $\mathbf{E} \mathbf{t}_{\mathrm{f} / C}$, on a un isomorphisme dans $E$

$$
\left(C^{\prime} \rightarrow X\right) \times G \simeq\left(C^{\prime} \rightarrow X\right) \times_{(C \rightarrow X)}\left(C^{\prime} \rightarrow X\right) .
$$

On note encore $G$ le faisceau constant de $\widetilde{E}$ de valeur $G$. Le morphisme $\left(C^{\prime} \rightarrow X\right)^{a} \rightarrow(C \rightarrow X)^{a}$ est alors un torseur sous $G$ de $\widetilde{E}$ [22, III 1.4.1]. Par descente galoisienne (cf. [7, 6.2.B]), la catégorie des données de descente $\mathbf{F}(\varpi)$ relativement au recouvrement $\varpi$ est équivalente à la catégorie $\widetilde{E}_{G}^{\sharp}$, d'où la proposition.

Corollaire 9.5. Le foncteur $\Phi_{n}^{*}: \operatorname{Mod}\left(\overline{\mathscr{B}}_{n}\right) \rightarrow \operatorname{Mod}_{G}\left(\overline{\mathscr{B}}_{n}^{\sharp}\right)$ (9.3.4) est une équivalence de catégories.

9.6. Pour tout objet $U$ de $\mathbf{E} \mathbf{t}_{/ X}$, on pose $U_{\bar{\eta}}^{\prime}=U \times_{X} C^{\prime}$ et l'on note $\varphi_{U}: U_{\bar{\eta}}^{\prime} \rightarrow U_{\bar{\eta}}$ le morphisme canonique. Considérons le site fibré

$$
\pi^{\sharp}=\pi \circ \gamma: E^{\sharp} \rightarrow \mathbf{E}_{/ X},
$$

dont la fibre au-dessus de $U$ est le site $\mathbf{E}_{\mathbf{t}_{\mathrm{f}} / U_{\bar{\eta}}^{\prime}}$ (9.9.1). Pour tout faisceau $\mathscr{G}$ de $\widetilde{E}^{\sharp}$, on note $\mathscr{G}_{U}$ sa restriction à $\mathbf{E} \mathbf{t}_{\mathrm{f} / U_{\bar{\eta}}^{\prime}}$. Le topos $\widetilde{E}^{\sharp}$ est canoniquement équivalent au topos de Faltings associé au morphisme canonique $\left(C^{\prime} \rightarrow X\right)$. La donnée d'un faisceau $\mathscr{G}$ de $\widetilde{E}^{\sharp}$ est alors équivalente à la donnée pour tout objet $U$ de $\mathbf{E} \mathbf{t}_{/ X}$ d'un faisceau $\mathscr{G}_{U}$ de $U_{\bar{\eta} \text {,fét }}^{\prime}$ vérifiant des conditions de comptabilité et de recollement (cf. [4, (VI.5.11.1)]). Comme $\varphi_{U}$ est un torseur sous $G$ pour la topologie étale de $U_{\bar{\eta}}$, le topos $U_{\bar{\eta} \text {,fét }}^{\prime}$ est muni d'une action de $G(9.1)$ induite par fonctorialité. D'après [4, VI.5.11], la donnée d'une action de $G \operatorname{sur} \mathscr{G}$ est équivalente à la donnée pour tout objet $U$ de $\mathbf{E} \mathbf{t}_{/ X}$ d'une action de $G$ sur $\mathscr{G}_{U}$ compatible aux morphismes de restriction. 
9.7. Soit $\mathscr{F}=\left\{U \mapsto \mathscr{F}_{U}\right\}_{U \in \mathbf{O b}\left(\mathbf{E ́}_{/ X}\right)}$ un préfaisceau sur $E$ tel que pour tout objet

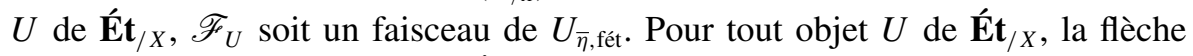
$\varphi_{U}: U_{\bar{\eta}}^{\prime} \rightarrow U_{\bar{\eta}}$ est un objet de $\mathbf{E} \mathbf{t}_{\mathrm{f} / U_{\bar{\eta}}}$. Le foncteur $\varphi_{U \text {,fét }}^{*}$ est donc un foncteur de restriction. On en déduit un isomorphisme canonique de préfaisceaux sur $E^{\sharp}$

$$
\mathscr{F} \circ \gamma \stackrel{\sim}{\rightarrow}\left\{U \mapsto \varphi_{U, \text { fét }}^{*}\left(\mathscr{F}_{U}\right)\right\},
$$

où $\gamma$ est le foncteur (9.2.1). Le faisceau associé à $\mathscr{F} \circ \gamma$ est isomorphe à $\Phi^{*}\left(\mathscr{F}^{a}\right)$. Le faisceau $\varphi_{U \text {,fét }}^{*}\left(\mathscr{F}_{U}\right)$ de $U_{\bar{\eta}}^{\prime}$,fét est muni d'une action de $G$ induite par $\varphi_{U \text {,fét }}^{*}$ fonctorielle en $U$. D'après 9.6, ils induisent une action de $G \operatorname{sur} \Phi^{*}\left(\mathscr{F}^{a}\right)$ qui coïncide avec celle de $\Phi^{*}\left(\mathscr{F}^{a}\right)$ induite par $\Phi^{*}$ (9.3.3).

9.8. On désigne par $\mathbf{P}$ la sous-catégorie pleine de $\mathbf{E}_{/ X}$ formée des schémas affines. On munit $\mathbf{P}$ de la topologie induite par celle de $\mathbf{E} \mathbf{t}_{/ X}$. Les objets de la sous-catégorie $\mathbf{P}$ forment alors une famille topologiquement génératrice du site $\mathbf{E ́}_{/ X}$. On désigne par

$$
\pi_{\mathbf{P}}^{\sharp}: E_{\mathbf{P}}^{\sharp} \rightarrow \mathbf{P}
$$

le site fibré déduit de $\pi^{\sharp}$ (9.6.1) par changement de base par $\mathbf{P} \rightarrow \mathbf{E ́}_{/ X}$. On munit $E_{\mathbf{P}}^{\sharp}$ de la topologie co-évanescente définie par $\pi_{\mathbf{P}}^{\sharp}$ et on note $\widetilde{E}_{\mathbf{P}}^{\sharp}$ le topos des faisceaux d'ensembles sur $E_{\mathbf{P}}$. D'après [4, VI.5.21 et VI.5.22], la topologie de $E_{\mathbf{P}}^{\sharp}$ est induite par celle de $E^{\sharp}$ au moyen du foncteur de projection canonique $E_{\mathbf{P}}^{\sharp} \rightarrow E^{\sharp}$ et celui-ci induit par restriction une équivalence de catégories

$$
\widetilde{E}^{\sharp} \stackrel{\sim}{\rightarrow} \widetilde{E}_{\mathbf{P}}^{\sharp}
$$

On désigne par $\widehat{E}_{\mathbf{P}}^{\sharp}$ la catégorie des préfaisceaux d'ensembles sur $E_{\mathbf{P}}$. Comme $E_{\mathbf{P}}^{\sharp}$ est une sous-catégorie topologiquement génératrice de $E^{\sharp}$, le foncteur 'faisceau associé' sur $E_{\mathbf{P}}^{\sharp}$ induit un foncteur que l'on note aussi

$$
\widehat{E}_{\mathbf{P}}^{\sharp} \rightarrow \widetilde{E}^{\sharp}, \quad \mathscr{F} \rightarrow \mathscr{F}^{a} .
$$

Soit $\mathscr{G}=\left\{W \mapsto \mathscr{G}_{W}\right\}\left(W \in \mathbf{O b}\left(\mathbf{E ́}_{/ X}\right)\right)$ un préfaisceau sur $E^{\sharp}, \mathscr{G}_{\mathbf{P}}=\{U \mapsto$ $\left.\mathscr{G}_{U}\right\}(U \in \mathbf{O b}(\mathbf{P}))$ l'objet de $\underset{\widehat{E}_{\mathbf{P}}^{\sharp}}{\sharp}$ obtenu en restreignant $\mathscr{G}$ à $E_{\mathbf{P}}^{\sharp}$. On en déduit un isomorphisme canonique de $\widetilde{E}^{\sharp}$

$$
\mathscr{G}_{\mathbf{P}}^{a} \stackrel{\sim}{\rightarrow} \mathscr{G}^{a} .
$$

9.9. Dans la suite de cette section, on suppose, de plus, qu'il existe un trait ( $S^{\prime}$, $\left.\eta^{\prime}\right)$ fini sur $(S, \eta)$ et un $\eta^{\prime}$-revêtement fini, galoisien et à fibres géométriquement réduites $\varphi: X^{\prime} \rightarrow X_{S^{\prime}}(5.4)$ tels que, en fixant un $\eta$-morphisme $\bar{\eta} \rightarrow \eta^{\prime}, X_{\bar{\eta}}^{\prime} \simeq C^{\prime}$ 
et que $\varphi_{\bar{\eta}}: X_{\bar{\eta}}^{\prime} \rightarrow X_{\bar{\eta}}$ s'identifie au morphisme $\psi$. On pose $\vec{X}^{\prime}=X^{\prime} \times_{S^{\prime}} \bar{S}$ et l'on note

$$
\hbar: \bar{X} \rightarrow X \quad \text { et } \bar{\varphi}: \vec{X}^{\prime} \rightarrow \bar{X}
$$

les morphismes canoniques.

Pour tout $(V \rightarrow U) \in \mathbf{O b}\left(E^{\sharp}\right)$, on pose $U^{\prime}=U \times_{X} X^{\prime}, \vec{U}^{\prime}=U^{\prime} \times_{X^{\prime}} \vec{X}^{\prime}$ et $\bar{U}^{V}$ (resp. $\vec{U}^{\prime V}$ ) la clôture intégrale de $\bar{U}$ (resp. $\vec{U}^{\prime}$ ) dans $V$. Comme $\vec{U}^{\prime}$ est fini sur $\bar{U}$, on a un isomorphisme canonique $\vec{U}^{\prime V} \stackrel{\sim}{\rightarrow} \bar{U}^{V}$. Comme $\Phi^{*}$ est le foncteur de restriction, on a $\Phi^{*}(\overline{\mathscr{B}})(V \rightarrow U) \simeq \overline{\mathscr{B}}(V \rightarrow U)$. On en déduit par (7.8.1) un isomorphisme fonctoriel en $(V \rightarrow U)$

$$
\overline{\mathscr{B}}^{\sharp}(V \rightarrow U) \stackrel{\sim}{\rightarrow} \Gamma\left(\vec{U}^{\prime V}, \mathscr{O}_{\vec{U}^{\prime V}}\right) .
$$

Pour tout $U \in \mathbf{O b}\left(\mathbf{E} \mathbf{t}_{/ X}\right)$, on pose $\overline{\mathscr{B}}_{U}^{\sharp}=\varphi_{U \text {,fét }}^{*}\left(\overline{\mathscr{B}}_{U}\right)(7.8 .2)$ et $\overline{\mathscr{B}}_{U, n}^{\sharp}=$ $\overline{\mathscr{B}}_{U}^{\sharp} / p^{n} \overline{\mathscr{B}}_{U}^{\sharp}$ qui est isomorphe à $\varphi_{U, \text { fét }}^{*}\left(\overline{\mathscr{B}}_{U, n}\right)$ (7.8.4). En vertu de (9.9.2), on a un homomorphisme d'anneaux de $U_{\bar{\eta}}^{\prime}$,ét

$$
\mathscr{O}_{\vec{X}^{\prime}}\left(\vec{U}^{\prime}\right) \rightarrow \overrightarrow{\mathscr{B}}_{U}^{\sharp},
$$

où la source désigne le faisceau constant de valeur $\mathscr{O}_{\vec{X}^{\prime}}\left(\vec{U}^{\prime}\right)$. Comme l'action de $G$ sur $C^{\prime}$ s'étend en une action de $G \operatorname{sur} \vec{X}^{\prime}$, l'anneau $\left.\mathscr{O}_{\vec{X}^{\prime}}, \vec{U}^{\prime}\right)$ est muni d'une action de $G$ telle que l'homomorphisme (9.9.3) soit $G$-équivariant. D'après 7.8 et (9.7.1), l'anneau $\overline{\mathscr{B}}_{n}^{\sharp}$ est isomorphe au faisceau sur $E^{\sharp}$ associé au préfaisceau

$$
\left\{U \rightarrow \overline{\mathscr{B}}_{U, n}^{\sharp}\right\} \quad U \in \mathbf{O b}\left(\mathbf{\mathbf { E }} \mathbf{t}_{/ X}\right) .
$$

9.10. Comme $\widetilde{E}^{\sharp}$ est isomorphe au topos de Faltings associé au morphisme canonique $C^{\prime} \rightarrow X$, on a un morphisme canonique [4, (VI.10.6.3)]

$$
\beta^{\sharp}: \widetilde{E}^{\sharp} \rightarrow C_{\text {fét }}^{\prime}
$$

qui rend commutatif le diagramme (cf. [4, (VI.10.12.6)])

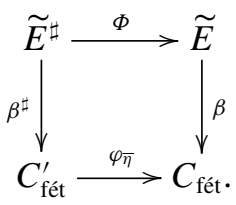

Soit $n$ un entier $\geqslant 1$. On désigne par $\beta_{n}^{\sharp}:\left(\widetilde{E}_{s}^{\sharp}, \overline{\mathscr{B}}_{n}^{\sharp}\right) \rightarrow\left(C_{\text {fét }}^{\prime}, \mathfrak{o}_{n}\right)$ le morphisme de topos annelés défini par le composé $\beta^{\sharp} \circ \delta^{\sharp}(9.2 .3)$ et le morphisme canonique 
$\mathfrak{o}_{n} \rightarrow \beta_{*}^{\sharp}\left(\overline{\mathscr{B}}_{n}^{\sharp}\right)$. Par des arguments similaires à ceux de 8.3 , on en déduit un diagramme commutatif

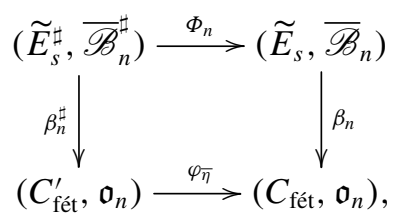

à isomorphisme près, qui commute aux actions de $G \operatorname{sur}\left(\widetilde{E}_{s}^{\sharp}, \overline{\mathscr{B}}_{n}^{\sharp}\right)$ et $\left(C_{\text {fét }}^{\prime}, \mathfrak{o}_{n}\right)$.

THÉORÈME 9.11. Soit X une S-courbe semi-stable, $\bar{x}$ un point géométrique de $X_{\bar{\eta}}$ et $\mathcal{F}$ un fibré vectoriel sur $\stackrel{\check{X}}{X}=X \times_{S} \check{\bar{S}}$ (2.1). Supposons qu'il existe un entier $n \geqslant 1$, un trait $\left(S^{\prime}, \eta^{\prime}\right)$ fini sur $(S, \eta)$ et un $\eta^{\prime}$-revêtement fini, galoisien et à fibres géométriquement réduites $\varphi: X^{\prime} \rightarrow X_{S^{\prime}}$ tels que, fixant un $\eta$-morphisme $\bar{\eta} \rightarrow \eta^{\prime}$ et reprenant les notations de (9.9.1), $\bar{\varphi}_{n}^{*}\left(\mathcal{F}_{n}\right)$ soit libre de type fini. Alors, on a un isomorphisme $\overline{\mathscr{B}}_{n}$-linéaire

$$
\gamma_{n}: \sigma_{n}^{*}\left(\mathcal{F}_{n}\right) \stackrel{\sim}{\rightarrow} \beta_{n}^{*}\left(\mathbb{V}_{n}(\mathcal{F})\right)
$$

où $\mathbb{V}_{n}(\mathcal{F})$ est la $\mathfrak{o}_{n}$-représentation de $\pi_{1}\left(X_{\bar{\eta}}, \bar{x}\right)$ associée à $\mathcal{F}(6.7)$, $\sigma_{n}$ est le morphisme de topos annelés (7.11.4) et $\beta_{n}^{*}$ est le foncteur (8.6.1).

Preuve. Pour tout objet $U$ de $\mathbf{E} \mathbf{t}_{/ X}$, on pose $U^{\prime}=U \times_{X} X^{\prime}, U_{s}^{\prime}=U^{\prime} \times_{S^{\prime}} s$ et $U_{\bar{\eta}}^{\prime}=U^{\prime} \times_{S^{\prime}} \bar{\eta}$. D'après (9.7.1) et [4, VI.5.34(ii) et VI.8.9], le $\overline{\mathscr{B}}_{n}^{\sharp}$-module $\Phi_{n}^{*}\left(\sigma_{n}^{*}\left(\mathcal{F}_{n}\right)\right)$ est isomorphe au faisceau associé au préfaisceau :

$$
\left\{U \mapsto \mathcal{F}_{n}\left(U_{s}\right) \otimes_{\mathscr{O}_{\bar{X}_{n}}\left(U_{s}\right)} \overline{\mathscr{B}}_{U, n}^{\sharp}, U \in \mathbf{O b}\left(\dot{\mathbf{E}}_{/ X}\right)\right\}
$$

où $\mathcal{F}_{n}\left(U_{s}\right)$ et $\mathscr{O}_{\bar{X}_{n}}\left(U_{s}\right)$ sont considérés comme des faisceaux constants de $U_{\bar{\eta}}^{\prime}$,fét . D'après (9.9.3), l'anneau $\overline{\mathscr{B}}_{U, n}^{\sharp}$ de $U_{\bar{\eta} \text {,fét }}^{\prime}$ est une $\mathscr{O}_{\vec{X}_{n}^{\prime}}\left(U_{s}^{\prime}\right)$-algèbre. Pour tout objet $U$ de $\mathbf{P}$ (9.8), on en déduit un isomorphisme $G$-équivariant de faisceaux de $U_{\bar{\eta}}^{\prime}$,ét

$$
\mathcal{F}_{n}\left(U_{s}\right) \otimes_{\mathscr{O}_{\bar{X}_{n}}\left(U_{s}\right)} \overline{\mathscr{B}}_{U, n}^{\sharp} \stackrel{\sim}{\rightarrow} \bar{\varphi}_{n}^{*}\left(\mathcal{F}_{n}\right)\left(U_{s}^{\prime}\right) \otimes_{\mathscr{O}_{\bar{X}_{n}^{\prime}}\left(U_{s}^{\prime}\right)} \overline{\mathscr{B}}_{U, n}^{\sharp},
$$

où les actions de $G$ sur $\mathcal{F}_{n}\left(U_{s}\right)$ et $\mathscr{O}_{\bar{X}_{n}}\left(U_{s}\right)$ sont triviales, et $\bar{\varphi}_{n}^{*}\left(\mathcal{F}_{n}\right)\left(U_{s}^{\prime}\right)$ et $\mathscr{O}_{\vec{X}_{n}^{\prime}}\left(U_{s}^{\prime}\right)$ sont considérés comme des faisceaux constants de $U_{\bar{\eta}}^{\prime}$,fét munis des actions de $G$ induites par $\bar{\varphi}_{n}^{*}$. On note $M_{U}$ le but de (9.11.3). 
On pose $\mathbb{L}_{n}(\mathcal{F})=\mu_{\bar{x}}\left(\mathbb{V}_{n}(\mathcal{F})\right)$ le $\mathfrak{o}_{n}$-module localement libre de type fini de

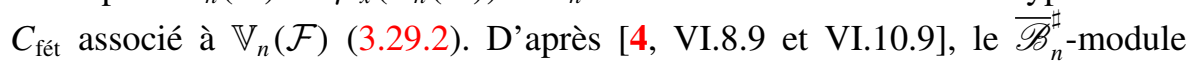
$\Phi_{n}^{*}\left(\beta_{n}^{*}\left(\mathbb{L}_{n}(\mathcal{F})\right)\right) \simeq\left(\beta_{n}^{\sharp}\right)^{*}\left(\varphi_{\bar{\eta}}^{*}\left(\mathbb{L}_{n}(\mathcal{F})\right)\right)$ est isomorphe au faisceau associé au préfaisceau

$$
\left\{U \mapsto h_{U, \text { fét }}^{*}\left(\varphi_{\bar{\eta}, \text { fét }}^{*}\left(\mathbb{L}_{n}(\mathcal{F})\right)\right) \otimes_{\mathfrak{o}_{n}} \overline{\mathscr{B}}_{U, n}^{\sharp}, U \in \mathbf{O b}\left(\mathbf{\mathbf { E }} \mathbf{t}_{/ X}\right)\right\},
$$

où $h_{U}^{\prime}: U_{\bar{\eta}}^{\prime} \rightarrow C^{\prime}$ est le morphisme canonique. On pose

$$
N_{U}=h_{U, \text { fét }}^{*}\left(\varphi_{\bar{\eta}, \text { fét }}^{*}\left(\mathbb{L}_{n}(\mathcal{F})\right)\right) \otimes_{\mathfrak{o}_{n}} \overline{\mathscr{B}}_{U, n}^{\sharp}
$$

qui est muni d'une action de $G$ fonctorielle en $U$ (cf. 9.7).

Comme $\bar{\varphi}_{n}^{*}\left(\mathcal{F}_{n}\right)$ est un $\mathscr{O}_{\vec{X}_{n}^{\prime}}$-module libre de type fini, d'après (6.7.3) et 6.8, on a un isomorphisme $G$-équivariant de $\mathfrak{o}_{n}$-modules de $C_{\text {fét }}^{\prime}$

$$
\bar{\varphi}_{n}^{*}\left(\mathcal{F}_{n}\right)\left(X_{s}^{\prime}\right) \simeq \varphi_{\bar{\eta} \text {, fét }}^{*}\left(\mathbb{L}_{n}(\mathcal{F})\right),
$$

où $\bar{\varphi}_{n}^{*}\left(\mathcal{F}_{n}\right)\left(X_{s}^{\prime}\right)$ est considéré comme le faisceau constant de $C_{\text {fét }}^{\prime}$ muni de l'action de $G$ induite par $\bar{\varphi}_{n}^{*}$. On a un isomorphisme $G$-équivariant et fonctoriel en $U$ de $\mathscr{O}_{\vec{X}_{n}^{\prime}}\left(U_{s}^{\prime}\right)$-modules

$$
\bar{\varphi}_{n}^{*}\left(\mathcal{F}_{n}\right)\left(U_{s}^{\prime}\right) \stackrel{\sim}{\rightarrow} \bar{\varphi}_{n}^{*}\left(\mathcal{F}_{n}\right)\left(X_{s}^{\prime}\right) \otimes_{\mathfrak{o}_{n}} \mathscr{O}_{\vec{X}_{n}^{\prime}}\left(U_{s}^{\prime}\right) .
$$

On en déduit par (9.11.5) un isomorphisme $G$-équivariant et fonctoriel en $U$ de $\mathscr{O}_{\vec{X}_{n}^{\prime}}\left(U_{s}^{\prime}\right)$-modules de $U_{\bar{\eta}}^{\prime}$,fét

$$
\bar{\varphi}_{n}^{*}\left(\mathcal{F}_{n}\right)\left(U_{s}^{\prime}\right) \simeq h_{U, \text { fét }}^{*}\left(\varphi_{\bar{\eta}, \text { fét }}^{*}\left(\mathbb{L}_{n}(\mathcal{F})\right)\right) \otimes_{\mathfrak{o}_{n}} \mathscr{O}_{\bar{X}_{n}^{\prime}}\left(U_{s}^{\prime}\right) .
$$

En vertu de (9.11.3) et (9.11.4), on en déduit, pour tout objet $U$ de $\mathbf{P}$, un isomorphisme $G$-équivariant et fonctoriel en $U$ de $\overline{\mathscr{B}}_{U, n}^{\sharp}$-modules de $U_{\bar{\eta}}^{\prime}$,fét

$$
M_{U} \stackrel{\sim}{\rightarrow} N_{U}
$$

D'après 9.7 et 9.8 , on en déduit un isomorphisme $G$-équivariant de $\overline{\mathscr{B}}_{n}^{\sharp}$-modules

$$
\Phi_{n}^{*}\left(\sigma_{n}^{*}\left(\mathcal{F}_{n}\right)\right) \stackrel{\sim}{\rightarrow} \Phi_{n}^{*}\left(\beta_{n}^{*}\left(\mathbb{L}_{n}(\mathcal{F})\right)\right) .
$$

Le théorème résulte alors de 9.5 .

Remarque 9.12. Pour tout entier $1 \leqslant m \leqslant n$, on a un isomorphisme $\overline{\mathscr{B}}_{m}$ linéaire $\gamma_{m}: \sigma_{m}^{*}\left(\mathcal{F}_{m}\right) \stackrel{\sim}{\rightarrow} \beta_{m}^{*}\left(\mathbb{V}_{m}(\mathcal{F})\right)$. En vertu de la preuve, les isomorphismes $\gamma_{n}$ et $\gamma_{m}$ sont compatibles. 


\section{Fibrés vectoriels et représentations de Weil-Tate}

10.1. Soit $X$ une $S$-courbe semi-stable, $\bar{x}$ un point géométrique de $X_{\bar{\eta}}$. On reprend les notations de la Section 7 pour $X$. Rappelons que l'on a des morphismes de topos annelés (cf. 7.12 et 7.13)

$$
\begin{aligned}
& \left(X_{s, \text { ét }}, \mathscr{O}_{\bar{X}_{n}}\right) \stackrel{\sigma_{n}}{\leftarrow}\left(\widetilde{E}_{s}, \overline{\mathscr{B}}_{n}\right) \stackrel{\beta_{n}}{\rightarrow}\left(X_{\bar{\eta}, \text { fét }}, \mathfrak{o}_{n}\right), \quad \forall n \geqslant 1 \\
& \left(X_{s, \text { ét }}^{\mathbb{N}^{\circ}}, \mathscr{O}_{\bar{X}}\right) \stackrel{\breve{\sigma}}{\leftarrow}\left(\widetilde{E}_{s}^{\mathbb{N}^{\circ}}, \breve{\breve{B}}\right) \stackrel{\breve{\beta}}{\rightarrow}\left(X_{\bar{\eta}, \text { fét }}^{\mathbb{N}^{\circ}}, \breve{\mathfrak{o}}\right) .
\end{aligned}
$$

On reprend les notations de 8.6 pour les foncteurs $\beta_{n}^{*}, \breve{\beta}^{*}$ et $\breve{\beta}_{\mathbb{Q}}^{*}$.

On désigne par $\mathfrak{X}$ le schéma formel complété $p$-adique de $\bar{X}$. Rappelons que $\mathfrak{X}$ est de présentation finie sur $\mathscr{S}=\operatorname{Spf}(\mathfrak{o})$ et que l'on a un morphisme de topos annelés (7.12.4)

$$
\mathrm{T}:\left(\widetilde{E}_{s}^{\mathbb{N}^{\circ}}, \breve{\mathscr{\mathscr { B }}}\right) \rightarrow\left(X_{s, \mathrm{zar}}, \mathscr{O}_{\mathfrak{X}}\right)
$$

Pour tout $\mathscr{O}_{\mathfrak{X}}$-module cohérent $\mathscr{F}$ de $X_{s, \text { zar }}$ et tout entier $n \geqslant 1$, on note $\mathscr{F}_{n}=$ $\mathscr{F} / p^{n} \mathscr{F}$ que l'on considère comme faisceau de $X_{s, \text { zar }}$ ou de $X_{s, \text { ét. }}$ D'après [4, (III.11.1.12)], on a un isomorphisme canonique

$$
\breve{\sigma}^{*}\left(\left(\mathscr{F}_{n}\right)_{n \geqslant 1}\right) \simeq \mathrm{T}^{*}(\mathscr{F}) .
$$

Le foncteur $\mathrm{T}_{*}$ induit un foncteur additif et exact à gauche que l'on note encore

$$
\mathrm{T}_{*}: \operatorname{Mod}_{\mathbb{Q}}(\overline{\mathscr{B}}) \rightarrow \operatorname{Mod}\left(\mathscr{O}_{\mathfrak{X}}\left[\frac{1}{p}\right]\right) .
$$

D'après (4.1.2), le foncteur $\mathrm{T}^{*}$ induit un foncteur additif que l'on note encore (7.15)

$$
\mathrm{T}^{*}: \operatorname{Mod}^{\mathrm{coh}}\left(\mathscr{O}_{\mathfrak{X}}\left[\frac{1}{p}\right]\right) \rightarrow \operatorname{Mod}_{\mathbb{Q}}^{\mathrm{atf}}\left(\frac{\breve{\mathscr{B}}}{)} .\right.
$$

DÉFINITION 10.2. (i) Soit $n$ un entier $\geqslant 1, \mathcal{F}_{n}$ un fibré vectoriel sur $\bar{X}_{n}$ et $\mathcal{V}_{n}$ une $\mathfrak{o}_{n}$-représentation de $\pi_{1}\left(X_{\bar{\eta}}, \bar{x}\right)(3.18)$. On dit que $\mathcal{F}_{n}$ et $\mathcal{V}_{n}$ sont $\overline{\mathscr{B}}_{n}{ }^{-}$ associés s'il existe un isomorphisme $\overline{\mathscr{B}}_{n}$-linéaire

$$
\sigma_{n}^{*}\left(\mathcal{F}_{n}\right) \stackrel{\sim}{\rightarrow} \beta_{n}^{*}\left(\mathcal{V}_{n}\right)
$$

(ii) Soit $\mathscr{F}$ un $\mathscr{O}_{\mathfrak{X}}\left[\frac{1}{p}\right]$-module localement projectif de type fini (2.9) et $V$ une C-représentation continue de $\pi_{1}\left(X_{\bar{\eta}}, \bar{x}\right)$ (3.18). On dit que $\mathscr{F}$ et $V$ sont $\breve{\mathscr{B}}_{\mathbb{Q}^{-}}$ associés s'il existe un isomorphisme $\breve{\mathscr{B}}_{\mathbb{Q}}$-linéaire

$$
\mathrm{T}^{*}(\mathscr{F}) \stackrel{\sim}{\rightarrow} \breve{\beta}_{\mathbb{Q}}^{*}(V) .
$$


DÉFINITION 10.3. (i) On dit qu'un $\mathscr{O}_{\mathfrak{X}}\left[\frac{1}{p}\right]$-module localement projectif de type fini $\mathscr{F}$ est de Weil-Tate s'il est $\breve{\mathscr{B}}_{\mathbb{Q}}$-associé à une $\mathfrak{C}$-représentation continue de $\pi_{1}\left(X_{\bar{\eta}}, \bar{x}\right)$.

(ii) On dit qu'une $\mathfrak{C}$-représentation continue $V$ de $\pi_{1}\left(X_{\bar{\eta}}, \bar{x}\right)$ est de Weil-Tate relativement à $X$ si elle est $\breve{\mathscr{B}}_{\mathbb{Q}^{-}}$associée à un $\mathscr{O}_{\mathfrak{X}}\left[\frac{1}{p}\right]$-module localement projectif de type fini.

On désigne par $\operatorname{Mod}^{\mathrm{WT}}\left(\mathscr{O}_{\mathfrak{X}}\left[\frac{1}{p}\right]\right)$ la sous-catégorie pleine de $\operatorname{Mod}^{\mathrm{coh}}\left(\mathscr{O}_{\mathfrak{X}}\left[\frac{1}{p}\right]\right)$ formée des $\mathscr{O}_{\mathfrak{X}}\left[\frac{1}{p}\right]$-modules de Weil-Tate et par $\operatorname{Rep}_{\mathfrak{C}}^{\mathrm{WT} / X}\left(\pi_{1}\left(X_{\bar{\eta}}, \bar{x}\right)\right)$ la souscatégorie pleine de $\operatorname{Rep}_{\mathfrak{C}}^{\text {cont }}\left(\pi_{1}\left(X_{\bar{\eta}}, \bar{x}\right)\right)$ formée des $\mathfrak{C}$-représentations de Weil-Tate relativement à $X$.

REMARQue 10.4. On dit qu'un fibré vectoriel $\mathcal{F}$ sur $\check{\bar{X}}=X \times_{S} \stackrel{\check{S}}{\bar{S}}(2.1)$ est de Weil-Tate si le $\mathscr{O}_{\mathfrak{X}}\left[\frac{1}{p}\right]$-module $\widehat{\mathcal{F}}\left[\frac{1}{p}\right]$ est de Weil-Tate. D'après $(10.1 .2)$, il revient au même de dire qu'il existe une $\mathfrak{C}$-représentation continue $V$ de $\pi_{1}\left(X_{\bar{\eta}}, \bar{x}\right)$ et un isomorphisme $\breve{\mathscr{B}}_{\mathbb{Q}}$-linéaire

$$
\left(\breve{\sigma}^{*}\left(\left(\mathcal{F}_{n}\right)_{n \geqslant 1}\right)\right)_{\mathbb{Q}} \stackrel{\sim}{\rightarrow} \breve{\beta}_{\mathbb{Q}}^{*}(V) .
$$

Proposition 10.5. Soit $X$ une $S$-courbe semi-stable, $\bar{x}$ un point géométrique de $X_{\bar{\eta}}, n$ un entier $\geqslant 1$ et $\mathcal{F}$ un fibré vectoriel de Deninger-Werner sur $\check{\bar{X}}$ (6.3). Alors, il existe un trait $S^{\prime}$ fini sur $S$, un $S^{\prime}$-modèle semi-stable et régulier $X^{\prime}$ de $X_{\bar{\eta}}(10.15)$ et un $\eta^{\prime}$-revêtement $\varphi: X^{\prime} \rightarrow X_{S^{\prime}}$ tels que $\bar{\varphi}_{n}^{*}\left(\mathcal{F}_{n}\right)$ et $\mathbb{V}_{\check{X}^{\prime}, n}(\mathcal{F})$ soient $\overline{\mathscr{B}}_{n}^{\prime}$-associés, où $\left(\widetilde{E}_{s}^{\prime}, \overline{\mathscr{B}}_{n}^{\prime}\right)$ désigne le topos annelé de Faltings associé au schéma $X^{\prime}$ au-dessus de $S^{\prime}$ et $\mathbb{V}_{\check{X}_{\bar{X}}, n}(\mathcal{F})$ est la $\mathfrak{o}_{n}$-représentation de $\pi_{1}\left(X_{\bar{\eta}}, \bar{x}\right)$ associée à $\mathcal{F}$ par (6.9.2).

Preuve. Quitte à remplacer $S$ par une extension finie, d'après 5.11(iii), il existe un $S$-modèle semi-stable $X^{\prime}$ de $X_{\bar{\eta}}$ et un $\eta$-revêtement $\varphi: X^{\prime} \rightarrow X$ tels que $\bar{\varphi}_{n}^{*}\left(\mathcal{F}_{n}\right)$ soit trivialisé par un $\eta$-revêtement semi-stable, fini et galoisien $Y^{\prime} \rightarrow X^{\prime}$. D'après 9.11, $\bar{\varphi}_{n}^{*}\left(\mathcal{F}_{n}\right)$ et $\mathbb{V}_{\bar{X}^{\prime}, n}\left(\bar{\varphi}^{*}(\mathcal{F})\right)$ sont $\overline{\mathscr{B}}_{n}^{\prime}$-associés. D'après (5.8), (8.3.13) et (8.3.14), on peut supposer que $X$ est régulier. La proposition s'ensuit alors compte tenu de 6.10(ii).

Proposition 10.6. Soit X une $S$-courbe propre et lisse, $\bar{x}$ un point géométrique de $X_{\bar{\eta}}$ et $\mathcal{L}$ un fibré en droites de classe appartenant à $\operatorname{Pic}_{\overline{\bar{X}} / \overline{\bar{S}}}^{0}(\overline{\bar{S}})$. Pour tout entier $n \geqslant 1$, on a associé à $\mathcal{L}$ une $\mathfrak{o}_{n}$-représentation $\mathbb{V}_{n}(\mathcal{L})$ de $\pi_{1}\left(X_{\bar{\eta}}, \bar{x}\right)(6.7)$ et une 
o-représentation continue $\mathbb{V}(\mathcal{L})$ de $\pi_{1}\left(X_{\bar{\eta}}, \bar{x}\right)$ (6.10.1). Alors, $\mathcal{L}_{n}$ et $\mathbb{V}_{n}(\mathcal{L})$ sont $\overline{\mathscr{B}}_{n}$-associés; et $\widehat{\mathcal{L}}\left[\frac{1}{p}\right]$ et $\mathbb{V}(\mathcal{L})\left[\frac{1}{p}\right](3.21)$ sont $\breve{\mathscr{B}}_{\mathbb{Q}^{-}}$associés.

Preuve. Soit un entier $n \geqslant 1$. D'après 6.5 et 9.11 , on a un isomorphisme

$$
\gamma_{n}: \sigma_{n}^{*}\left(\mathcal{L}_{n}\right) \stackrel{\sim}{\rightarrow} \beta_{n}^{*}\left(\mathbb{V}_{n}(\mathcal{L})\right),
$$

d'où le premier énoncé. En vertu de 9.12, les isomorphismes $\left(\gamma_{n}\right)_{n \geqslant 1}(10.6 .1)$ sont compatibles. La proposition s'ensuit compte tenu de (10.1.2).

Proposition 10.7. Soit X une $S$-courbe semi-stable et régulière et $\bar{x}$ un point géométrique de $X_{\bar{\eta}}$.

(i) La restriction du foncteur $\mathrm{T}^{*}(10.1 .4) \grave{a} \mathbf{M o d}^{\mathrm{WT}}\left(\mathscr{O}_{\mathfrak{X}}\left[\frac{1}{p}\right]\right)$ se factorise à travers la sous-catégorie pleine $\operatorname{Mod}_{\mathbb{Q}}^{\mathrm{pltf}} \frac{\breve{\mathscr{B}}}{(8.5)}$ de $\operatorname{Mod}_{\mathbb{Q}}^{\mathrm{atf}} \frac{\breve{\mathscr{B}})}{}$.

(ii) Pour tout objet $\mathscr{F}$ de $\operatorname{Mod}^{\mathrm{WT}}\left(\mathscr{O}_{\mathfrak{X}}\left[\frac{1}{p}\right]\right)$, on a un isomorphisme $\breve{\mathscr{B}}_{\mathbb{Q}}$-linéaire, canonique et fonctoriel

$$
\mathrm{T}^{*}(\mathscr{F}) \stackrel{\sim}{\rightarrow} \breve{\beta}_{\mathbb{Q}}^{*}\left(\mathscr{W}_{\mathbb{Q}}\left(\mathrm{T}^{*}(\mathscr{F})\right)\right)
$$

où $\mathscr{W}_{\mathbb{Q}}: \operatorname{Mod}_{\mathbb{Q}}^{\mathrm{pltf}}(\breve{\mathscr{B}}) \rightarrow \operatorname{Rep}_{\mathfrak{C}}^{\text {cont }}\left(\pi_{1}\left(X_{\bar{\eta}}, \bar{x}\right)\right)$ est le foncteur $(8.14 .2)$.

Preuve. Soit $\mathscr{F}$ un objet de $\operatorname{Mod}^{\mathrm{WT}}\left(\mathscr{O}_{\mathfrak{X}}\left[\frac{1}{p}\right]\right)$. Choisissons un objet $V$ de $\operatorname{Rep}_{\mathfrak{C}}^{\text {cont }}\left(\pi_{1}\left(X_{\bar{\eta}}, \bar{x}\right)\right)$ et un isomorphisme $\breve{\breve{B}}_{\mathbb{Q}}$-linéaire

$$
\tau: \mathrm{T}^{*}(\mathscr{F}) \simeq \breve{\beta}_{\mathbb{Q}}^{*}(V)
$$

L'assertion (i) résulte alors de 8.7. D'après 8.17(ii), on a un isomorphisme $\breve{\mathscr{B}}_{\mathbb{Q}^{-}}$ linéaire canonique et fonctoriel

$$
\breve{\beta}_{\mathbb{Q}}^{*}(V) \stackrel{\sim}{\rightarrow} \breve{\beta}_{\mathbb{Q}}^{*}\left(\mathscr{W}_{\mathbb{Q}}\left(\breve{\beta}_{\mathbb{Q}}^{*}(V)\right)\right)
$$

On en déduit, compte tenu de (10.7.2) et (10.7.3), un isomorphisme $\breve{\breve{B}}_{\mathbb{Q}}$-linéaire

$$
\mathrm{T}^{*}(\mathscr{F}) \stackrel{\sim}{\rightarrow} \breve{\beta}_{\mathbb{Q}}^{*}\left(\mathscr{W}_{\mathbb{Q}}\left(\mathrm{T}^{*}(\mathscr{F})\right)\right) .
$$

Montrons que cet isomorphisme ne dépend pas des choix de $V$ et de l'isomorphisme $\tau$ et qu'il est fonctoriel en $\mathscr{F}$. Il suffit de démontrer que, 
pour tous objets $V$ et $V^{\prime}$ de $\operatorname{Rep}_{\mathfrak{C}^{*}}^{\text {cont }}\left(\pi_{1}\left(X_{\bar{\eta}}, \bar{x}\right)\right)$ et tout morphisme $\breve{\breve{B}}_{\mathbb{Q}}$-linéaire $\theta: \breve{\beta}_{\mathbb{Q}}^{*}(V) \rightarrow \breve{\beta}_{\mathbb{Q}}^{*}\left(V^{\prime}\right)$, le diagramme

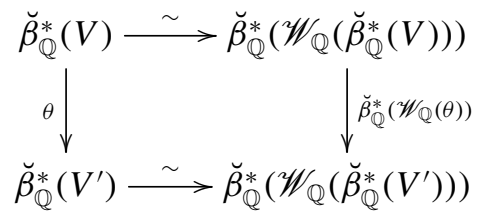

est commutatif. D'après la pleine fidélité du foncteur $\breve{\beta}_{\mathbb{Q}}^{*}(8.8), \theta$ est l'image d'un morphisme $\vartheta: V \rightarrow V^{\prime}$ de $\operatorname{Rep}_{\mathfrak{C}}^{\text {cont }}\left(\pi_{1}\left(X_{\bar{\eta}}, \bar{x}\right)\right)$ par $\breve{\beta}_{\mathbb{Q}}^{*}$. La commutativité de (10.7.4) résulte alors de la fonctorialité de (10.7.3).

COROllaire 10.8. Sous les hypothèses de 10.7, pour tout objet $\mathscr{F}$ de $\operatorname{Mod}^{\mathrm{WT}}\left(\mathscr{O}_{\mathfrak{X}}\left[\frac{1}{p}\right]\right)$, la $\mathfrak{C}$-représentation continue $\mathscr{W}_{\mathbb{Q}}\left(\mathrm{T}^{*}(\mathscr{F})\right)$ est de Weil-Tate relativement à $X$. On désigne par $\mathscr{V}_{\mathfrak{X}}$ le foncteur

$$
\begin{aligned}
\mathscr{V}_{\mathfrak{X}}: \operatorname{Mod}^{\mathrm{WT}}\left(\mathscr{O}_{\mathfrak{X}}\left[\frac{1}{p}\right]\right) & \rightarrow \operatorname{Rep}_{\mathfrak{C}}^{\mathrm{WT} / X}\left(\pi_{1}\left(X_{\bar{\eta}}, \bar{x}\right)\right) \\
\mathscr{F} & \mapsto \mathscr{W}_{\mathbb{Q}}\left(\mathrm{T}^{*}(\mathscr{F})\right) .
\end{aligned}
$$

Alors, $\mathscr{V}_{\mathfrak{X}}(\mathscr{F})$ et $\mathscr{F}$ sont $\breve{\mathscr{B}}_{\mathbb{Q}}$-associés.

Proposition 10.9. Soit $X$ une S-courbe semi-stable et régulière. On note $\mathbf{L P}^{\mathrm{tf}}\left(\mathscr{O}_{\mathfrak{X}}\left[\frac{1}{p}\right]\right)$ la sous-catégorie pleine de $\operatorname{Mod}^{\mathrm{coh}}\left(\mathscr{O}_{\mathfrak{X}}\left[\frac{1}{p}\right]\right)$ formée des $\mathscr{O}_{\mathfrak{X}}\left[\frac{1}{p}\right]$ modules localement projectifs de type fini.

(i) Pour tout objet $\mathscr{F}$ de $\mathbf{L P}^{\mathrm{tf}}\left(\mathscr{O}_{\mathfrak{X}}\left[\frac{1}{p}\right]\right)$, le morphisme d'adjonction (cf. [4, III.12.1])

$$
\mathscr{F} \rightarrow \mathrm{T}_{*}\left(\mathrm{~T}^{*}(\mathscr{F})\right)
$$

est un isomorphisme.

(ii) La restriction du foncteur $\mathrm{T}^{*}(10.1 .4)$ à $\mathbf{L} \mathbf{P}^{\mathrm{tf}}\left(\mathscr{O}_{\mathfrak{X}}\left[\frac{1}{p}\right]\right)$ est pleinement fidèle.

Preuve. (i) D'après [4, III.12.4(ii)], on a un isomorphisme déduit du morphisme d'adjonction

$$
\mathscr{F} \otimes_{\mathscr{O} \mathfrak{X}\left[\frac{1}{p}\right]} \mathrm{T}_{*}\left(\overline{\breve{\mathscr{B}}}_{\mathbb{Q}}\right) \stackrel{\sim}{\rightarrow} \mathrm{T}_{*}\left(\mathrm{~T}^{*}(\mathscr{\mathscr { F }})\right) .
$$

L'isomorphisme (10.9.1) résulte alors de l'isomorphisme [4, III.11.8]

$$
\mathscr{O}_{\mathfrak{X}}\left[\frac{1}{p}\right] \stackrel{\sim}{\rightarrow} \mathrm{T}_{*}(\stackrel{\breve{\mathscr{B}}}{\mathbb{Q}}) .
$$


(ii) Soit $\mathscr{G}_{1}$ et $\mathscr{G}_{2}$ deux $\mathscr{O}_{\mathfrak{X}}$-modules cohérents de $X_{s, \text { zar }}$ tels que $\mathscr{G}_{1}\left[\frac{1}{p}\right]$ et $\mathscr{G}_{2}\left[\frac{1}{p}\right]$ soient localement projectifs de type fini. Notons $\mathscr{G}=\mathscr{H}_{\text {om }} \mathscr{O}_{\mathfrak{X}}\left(\mathscr{G}_{1}, \mathscr{G}_{2}\right)$. Alors, $\mathscr{G}\left[\frac{1}{p}\right]$ est aussi localement projectif de type fini (2.9). Comme $\mathscr{G}_{1}$ est de présentation finie, on a un isomorphisme canonique

$$
\mathrm{T}^{*}(\mathscr{G}) \stackrel{\sim}{\rightarrow} \mathscr{H}_{0 m_{\breve{\mathscr{B}}}}\left(\mathrm{T}^{*}\left(\mathscr{G}_{1}\right), \mathrm{T}^{*}\left(\mathscr{G}_{2}\right)\right) .
$$

Considérons le morphisme composé

$$
f: \Gamma\left(X_{s, \mathrm{zar}}, \mathscr{G}\right) \rightarrow \Gamma\left(X_{s, \mathrm{zar}}, \mathrm{T}_{*}\left(\mathrm{~T}^{*}(\mathscr{G})\right)\right) \stackrel{\sim}{\rightarrow} \Gamma\left(\widetilde{E}_{s}^{\mathbb{N}^{\circ}}, \mathrm{T}^{*}(\mathscr{G})\right) .
$$

D'après (i) et 5.16(i), le morphisme $f \otimes_{\mathbb{Z}} \mathbb{Q}$ est un isomorphisme. On en déduit la pleine fidélité de $\mathrm{T}^{*}$.

Proposition 10.10. Soit X une S-courbe semi-stable et régulière, et $\bar{x}$ un point géométrique de $X_{\bar{\eta}}$. Pour toute $\mathfrak{C}$-représentation $V$ de Weil-Tate relativement à $X$, le $\mathscr{O}_{\mathfrak{X}}\left[\frac{1}{p}\right]$-module $\mathrm{T}_{*}\left(\breve{\beta}_{\mathbb{Q}}^{*}(V)\right)$ est de Weil-Tate. De plus, on a un isomorphisme $\breve{\mathscr{B}}_{\mathbb{Q}}$-linéaire, canonique et fonctoriel

$$
\breve{\beta}_{\mathbb{Q}}^{*}(V) \stackrel{\sim}{\rightarrow} \mathrm{T}^{*}\left(\mathrm{~T}_{*}\left(\breve{\beta}_{\mathbb{Q}}^{*}(V)\right)\right) .
$$

Preuve. Soit $V$ une $\mathfrak{C}$-représentation de $\pi_{1}\left(X_{\bar{\eta}}, \bar{x}\right)$ de Weil-Tate relativement à $X$. Choisissons un $\mathscr{O}_{\mathfrak{X}}\left[\frac{1}{p}\right]$-module de Weil-Tate $\mathscr{F}$ et un isomorphisme $\breve{\mathscr{B}}_{\mathbb{Q}}$-linéaire

$$
\tau: \breve{\beta}_{\mathbb{Q}}^{*}(V) \simeq \mathrm{T}^{*}(\mathscr{F})
$$

D'après $(10.9 .1), \mathrm{T}_{*}\left(\breve{\beta}_{\mathbb{Q}}^{*}(V)\right)$ est de Weil-Tate.

En vertu de (10.9.1), on a un isomorphisme $\breve{\mathscr{B}}_{\mathbb{Q}}$-linéaire, canonique et fonctoriel en $\mathscr{F}$

$$
\mathrm{T}^{*}(\mathscr{F}) \stackrel{\sim}{\rightarrow} \mathrm{T}^{*}\left(\mathrm{~T}_{*}\left(\mathrm{~T}^{*}(\mathscr{F})\right)\right)
$$

On en déduit, compte tenu de $(10.10 .2)$ et $(10.10 .3)$, un isomorphisme $\breve{\mathscr{B}}_{\mathbb{Q}^{-}}$ linéaire

$$
\breve{\beta}_{\mathbb{Q}}^{*}(V) \stackrel{\sim}{\rightarrow} \mathrm{T}^{*}\left(\mathrm{~T}_{*}\left(\breve{\beta}_{\mathbb{Q}}^{*}(V)\right)\right) .
$$

Montrons que cet isomorphisme ne dépend pas des choix de $\mathscr{F}$ et de l'isomorphisme $\tau$, et qu'il est fonctoriel en $V$. Il suffit de montrer que, pour tous $\mathscr{O}_{\mathfrak{X}}\left[\frac{1}{p}\right]$-modules localement projectifs de type fini $\mathscr{F}, \mathscr{F}^{\prime}$ et tout morphisme 
$\breve{\mathscr{B}}_{\mathbb{Q}}$-linéaire $\theta: \mathrm{T}^{*}(\mathscr{F}) \rightarrow \mathrm{T}^{*}\left(\mathscr{F}^{\prime}\right)$, le diagramme

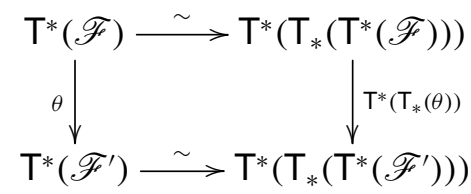

est commutatif. D'après la pleine fidélité du foncteur T* 10.9(ii), $\theta$ est l'image d'un morphisme $\vartheta: \mathscr{F} \rightarrow \mathscr{F}^{\prime}$ de $\mathbf{L P}^{\mathrm{tf}}\left(\mathscr{O}_{\mathfrak{X}}\left[\frac{1}{p}\right]\right)$ par $\mathrm{T}^{*}$. La commutativité de (10.10.4) résulte alors de la fonctorialité de (10.10.3) en $\mathscr{F}$.

Corollaire 10.11. Sous les hypothèses de 10.10, pour toute $\mathfrak{C}$ représentation $V$ de Weil-Tate relativement à $X$, le $\mathscr{O}_{\mathfrak{X}}\left[\frac{1}{p}\right]$-module $\mathrm{T}_{*}\left(\breve{\beta}_{\mathbb{Q}}^{*}(V)\right)$ est de Weil-Tate. On désigne par $\mathscr{T}_{\mathfrak{X}}$ le foncteur

$$
\begin{aligned}
\mathscr{T}_{\mathfrak{X}}: \operatorname{Rep}_{\mathfrak{C}}^{\mathrm{WT} / X}\left(\pi_{1}\left(X_{\bar{\eta}}, \bar{x}\right)\right) & \rightarrow \operatorname{Mod}^{\mathrm{WT}}\left(\mathscr{O}_{\mathfrak{X}}\left[\frac{1}{p}\right]\right) \\
V & \mapsto \mathrm{T}_{*}\left(\breve{\beta}_{\mathbb{Q}}^{*}(V)\right) .
\end{aligned}
$$

Alors, $V$ et $\mathscr{T}_{\mathfrak{X}}(V)$ sont $\breve{\breve{B}}_{\mathbb{Q}^{-}}$associés.

Proposition 10.12. Soit $X$ une $S$-courbe semi-stable et régulière, et $\bar{x}$ un point géométrique de $X_{\bar{\eta}}$. Les foncteurs $\mathscr{V}_{\mathfrak{X}}(10.8 .1)$ et $\mathscr{T}_{\mathfrak{X}}(10.11 .1)$ sont des équivalences de catégories quasi inverses l'une de l'autre.

Preuve. D'après 10.7(ii), pour tout objet $\mathscr{F} \operatorname{de} \operatorname{Mod}^{\mathrm{WT}}\left(\mathscr{O}_{\mathfrak{X}}\left[\frac{1}{p}\right]\right)$, on a un isomorphisme $\breve{\mathscr{B}}_{\mathbb{Q}}$-linéaire, canonique et fonctoriel

$$
\mathrm{T}^{*}(\mathscr{F}) \stackrel{\sim}{\rightarrow} \breve{\beta}_{\mathbb{Q}}^{*}\left(\mathscr{V}_{\mathfrak{X}}(\mathscr{F})\right)
$$

Composant l'isomorphisme canonique (10.9.1) et l'image de (10.12.1) par $\mathrm{T}_{*}$, on en déduit un isomorphisme fonctoriel

$$
\mathscr{F} \stackrel{\sim}{\rightarrow} \mathscr{T}_{\mathfrak{X}}\left(\mathscr{V}_{\mathfrak{X}}(\mathscr{F})\right) .
$$

D'autre part, d'après (10.10.1), pour tout objet $V$ de $\operatorname{Rep}_{\mathfrak{C}^{\mathrm{C}}}^{\mathrm{WT} / X}\left(\pi_{1}\left(X_{\bar{\eta}}, \bar{x}\right)\right)$, on a un isomorphisme $\breve{\breve{B}}_{\mathbb{Q}}$-linéaire, canonique et fonctoriel

$$
\breve{\beta}_{\mathbb{Q}}^{*}(V) \stackrel{\sim}{\rightarrow} \mathrm{T}^{*}\left(\mathscr{T}_{\mathfrak{X}}(V)\right)
$$


Composant l'isomorphisme canonique $V \stackrel{\sim}{\rightarrow} \mathscr{W}_{\mathbb{Q}}\left(\breve{\beta}_{\mathbb{Q}}^{*}(V)\right)(8.17($ ii $))$ et l'image de (10.12.3) par $\mathscr{W}_{\mathbb{Q}}$, on en déduit un isomorphisme fonctoriel

$$
V \stackrel{\sim}{\rightarrow} \mathscr{V}_{\mathfrak{X}}\left(\mathscr{T}_{\mathfrak{X}}(V)\right)
$$

10.13. Soit $X$ une $S$-courbe semi-stable et régulière, $\left(S^{\prime}, \eta^{\prime}\right)$ un trait fini sur $(S$, $\eta$ ) et $\varphi: X^{\prime} \rightarrow X_{S^{\prime}}$ un $\eta^{\prime}$-revêtement semi-stable et régulier. Soit $\bar{x}$ un $\bar{\eta}$-point de $X_{\bar{\eta}}$ et $\bar{x}^{\prime}$ un $\bar{\eta}$-point de $X_{\bar{\eta}}^{\prime}$ au-dessus de $\bar{x}$. On fixe un $\eta$-morphisme $\bar{\eta} \rightarrow \eta^{\prime}$ et par suite un $S$-morphisme $\bar{S} \rightarrow S^{\prime}$. On désigne par $\mathfrak{X}^{\prime}$ le schéma formel complété $p$-adique de $X \times_{S^{\prime}} \bar{S}$ et par $\mathfrak{g}: \mathfrak{X}^{\prime} \rightarrow \mathfrak{X}$ le morphisme de schémas formels induit par la projection canonique $X^{\prime} \rightarrow X_{S^{\prime}}$. Reprenant les notations de 8.3 pour $\varphi$, on désigne par

$$
\mathrm{T}^{\prime}:\left(\widetilde{E}_{s}^{\mathbb{N}^{\circ}}, \breve{\mathscr{B}}^{\prime}\right) \rightarrow\left(X_{s, \mathrm{zar}}^{\prime}, \mathscr{O}_{\mathfrak{X}^{\prime}}\right)
$$

le morphisme (10.1.1) relatif à $X^{\prime}$. On a alors un diagramme commutatif à isomorphismes près (cf. (8.3.14) et [4, III.(9.11.15)])

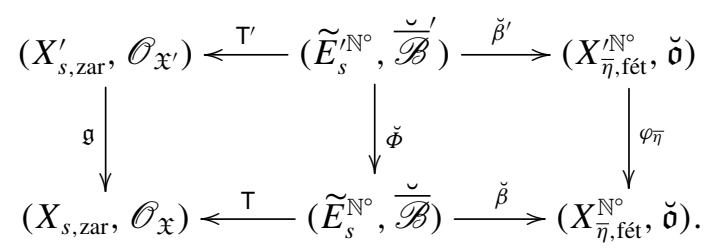

L'homomorphisme canonique $\pi_{1}\left(X_{\bar{\eta}}^{\prime}, \bar{x}^{\prime}\right) \rightarrow \pi_{1}\left(X_{\bar{\eta}}, \bar{x}\right)$ induit un foncteur

$$
\operatorname{Rep}_{\mathfrak{C}}^{\text {cont }}\left(\pi_{1}\left(X_{\bar{\eta}}, \bar{x}\right)\right) \rightarrow \operatorname{Rep}_{\mathfrak{C}}^{\text {cont }}\left(\pi_{1}\left(X_{\bar{\eta}}^{\prime}, \bar{x}^{\prime}\right)\right)
$$

Proposition 10.14. Conservons les notations de 10.13. Alors :

(i) l'image inverse d'un $\mathscr{O}_{\mathfrak{X}}\left[\frac{1}{p}\right]$-module de Weil-Tate par $\mathfrak{g}$ est un $\mathscr{O}_{\mathfrak{X}^{\prime}}\left[\frac{1}{p}\right]$-module de Weil-Tate;

(ii) toute $\mathfrak{C}$-représentation continue de Weil-Tate relativement à $X$ de $\pi_{1}\left(X_{\bar{\eta}}, \bar{x}\right)$ définit par restriction (10.13.3) une $\mathfrak{C}$-représentation continue de Weil-Tate relativement à $X^{\prime}$ de $\pi_{1}\left(X_{\bar{\eta}}^{\prime}, \bar{x}^{\prime}\right)$; 
(iii) on a des diagrammes commutatifs à isomorphismes près

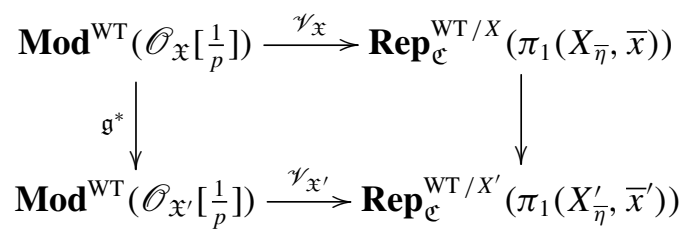

et

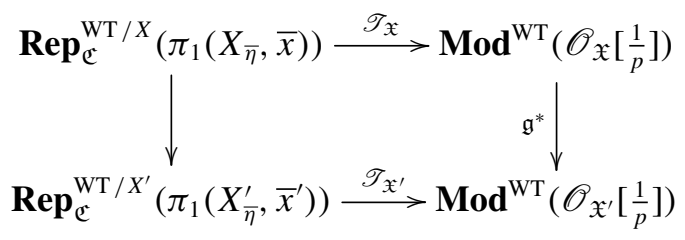

où $\mathscr{V}_{\mathfrak{X}^{\prime}}$ et $\mathscr{T}_{\mathfrak{X}^{\prime}}$ sont les foncteurs (10.8.1) et (10.11.1) relatifs à $X^{\prime}$.

Preuve. Soit $\mathscr{F}$ un $\mathscr{O}_{\mathfrak{X}}\left[\frac{1}{p}\right]$-module de Weil-Tate et $V$ une $\mathfrak{C}$-représentation de Weil-Tate associée. Il existe donc un isomorphisme $\breve{\mathscr{B}}_{\mathbb{Q}^{-}}$-linéaire

$$
\mathrm{T}^{*}(\mathscr{F}) \stackrel{\sim}{\rightarrow} \breve{\beta}_{\mathbb{Q}}^{*}(V)
$$

En vertu de (10.13.2), on en déduit un isomorphisme $\breve{\breve{B}}_{\mathbb{Q}}^{\prime}$-linéaire

$$
\mathrm{T}^{* *}\left(\mathfrak{g}^{*}(\mathscr{F})\right) \stackrel{\sim}{\rightarrow} \breve{\beta}_{\mathbb{Q}}^{\prime *}(V)
$$

d'où les assertions (i) et (ii).

Comme $\mathscr{V}_{\mathfrak{X}}=\mathrm{T}^{*} \circ \mathscr{W}_{\mathbb{Q}}$, la commutativité du diagramme (10.14.1) résulte de 8.15 et (10.13.2). La commutativité du diagramme (10.14.2) s'ensuit par 10.12, d'où l'assertion (iii).

10.15. Dans la suite de cette section, on fixe une $\bar{K}$-courbe propre et lisse $C$ et un point géométrique $\bar{x}$ de $C$. On pose $\check{C}=C \otimes_{\bar{K}} \mathfrak{C}$. Soit $S^{\prime}$ un trait fini sur $S$. Un $S^{\prime}$-modèle de $C$ est la donnée d'un $S$-morphisme $\bar{S} \rightarrow S^{\prime}$ et d'une $S^{\prime}$-courbe propre $X$ munie d'un isomorphisme $C \simeq X \times{ }_{S^{\prime}} \bar{\eta}$. Soit $X$ un $S^{\prime}$-modèle semi-stable et régulier de $C$. Reprenant les notations de 5.14, on a un foncteur pleinement fidèle en vertu de (5.15)

$$
J_{\mathfrak{X}}: \mathbf{L P}^{\mathrm{tf}}\left(\mathscr{O}_{\mathfrak{X}}\left[\frac{1}{p}\right]\right) \rightarrow \operatorname{Vect}_{\check{C}} .
$$


DÉFINITION 10.16. (i) On dit qu'un fibré vectoriel $F$ sur $\check{C}$ est de Weil-Tate s'il existe un trait $S^{\prime}$ fini sur $S$ et un $S^{\prime}$-modèle semi-stable et régulier $X$ de $C$ et un $\mathscr{O}_{\mathfrak{X}}\left[\frac{1}{p}\right]$-module de Weil-Tate $\mathscr{F}$ tels que $J \mathfrak{X}(\mathscr{F}) \simeq F(10.15 .1)$.

(ii) On dit qu'une $\mathfrak{C}$-représentation continue $V$ de $\pi_{1}(C, \bar{x})$ est de Weil-Tate s'il existe un trait $S^{\prime}$ fini sur $S$, un $S^{\prime}$-modèle semi-stable $X$ de $C$ tels que $V$ soit de Weil-Tate relativement à $X$.

On désigne par $\mathfrak{V}_{\breve{C}}^{\mathrm{WT}}$ la sous-catégorie pleine de Vect $_{\breve{C}}$ formée des fibrés vectoriels de Weil-Tate et par $\operatorname{Rep}_{\mathfrak{C}}^{\mathrm{WT}}\left(\pi_{1}(C, \bar{x})\right)$ la sous-catégorie pleine de $\operatorname{Rep}_{\mathfrak{C}}^{\text {cont }}\left(\pi_{1}(C, \bar{x})\right)$ formée des $\mathfrak{C}$-représentations continues de Weil-Tate. Soit $X$ un $S$-modèle semi-stable et régulier de $C$. La restriction du foncteur $J \mathfrak{X}$ (10.15.1) à $\operatorname{Mod}^{\mathrm{WT}}\left(\mathscr{O}_{\mathfrak{X}}\left[\frac{1}{p}\right]\right)$ induit un foncteur pleinement fidèle que l'on note encore

$$
J \mathfrak{X}: \operatorname{Mod}^{\mathrm{WT}}\left(\mathscr{O}_{\mathfrak{X}}\left[\frac{1}{p}\right]\right) \rightarrow \mathfrak{V}_{\check{C}}^{\mathrm{WT}} .
$$

Soit $S^{\prime}$ un trait fini sur $S$ et $X^{\prime}$ un $S^{\prime}$-modèle semi-stable et régulier de $C$ dominant $X_{S^{\prime}}$. Reprenons les notations de 10.13. En vertu de 10.14(i) et de la définition de $J \mathfrak{X}$ (5.14.2), on vérifie que le diagramme

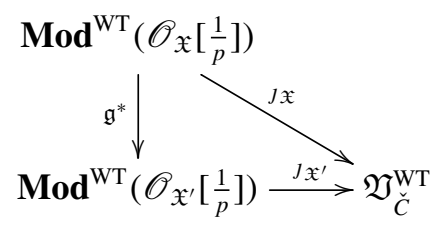

où $J \mathfrak{X}^{\prime}$ désigne le foncteur (10.16.1) relatif à $X^{\prime}$, est commutatif.

LEMme 10.17. (i) Pour tout morphisme $f$ de $\mathfrak{V}_{\check{C}}^{\mathrm{WT}}$, il existe un trait $S^{\prime}$ fini sur $S$ et un $S^{\prime}$-modèle semi-stable et régulier $X$ de $C$ tels que $f$ soit contenu dans l'image du foncteur $J \mathfrak{X}(10.16 .1)$.

(ii) Pour tout morphisme $f$ de $\operatorname{Rep}_{\mathfrak{C}}^{\mathrm{WT}}\left(\pi_{1}(C, \bar{x})\right)$, il existe un trait $S^{\prime}$ fini sur $S$ et un $S^{\prime}$-modèle semi-stable $X$ de $C$ tels que $f$ provienne de la sous-catégorie pleine $\operatorname{Rep}_{\mathfrak{C}}^{\mathrm{WT} / X}\left(\pi_{1}(C, \bar{x})\right)$ de $\operatorname{Rep}_{\mathfrak{C}}^{\mathrm{WT}}\left(\pi_{1}(C, \bar{x})\right)$.

Preuve. (i) Soit $F$ et $F^{\prime}$ deux fibrés vectoriels de Weil-Tate sur $\check{C}$. D'après 5.8, il existe un trait $S^{\prime}$ fini sur $S$ et un $S^{\prime}$-modèle $X$ semi-stable et régulier de $C$ tels que $F$ et $F^{\prime}$ soient les images des $\mathscr{O}_{\mathfrak{X}}\left[\frac{1}{p}\right]$-modules localement projectifs de type fini par $J_{\mathfrak{X}}$ (10.16.1). L'assertion résulte alors de la pleine fidélité de (10.16.1).

En calquant la démonstration de (i), on vérifie l'assertion (ii). 
10.18. En vertu de 10.14 et 10.17 , les foncteurs $\mathscr{V}_{\mathfrak{X}}$ fournissent un foncteur

$$
\mathscr{V}_{\check{C}}: \mathfrak{V}_{\check{C}}^{\mathrm{WT}} \rightarrow \operatorname{Rep}_{\mathfrak{C}}^{\mathrm{WT}}\left(\pi_{1}(C, \bar{x})\right)
$$

De même, les foncteurs $J_{\mathfrak{X}} \circ \mathscr{T}_{\mathfrak{X}}$ fournissent un foncteur :

$$
\mathscr{T}_{\check{C}}: \operatorname{Rep}_{\mathfrak{C}}^{\mathrm{WT}}\left(\pi_{1}(C, \bar{x})\right) \rightarrow \mathfrak{V}_{\check{C}}^{\mathrm{WT}}
$$

THÉORÈme 10.19. Les foncteurs $\mathscr{V}_{\check{C}}(10.18 .1)$ et $\mathscr{T}_{\check{C}}$ (10.18.2) sont des équivalences de catégories quasi inverses l'une de l'autre.

Preuve. Pour tout objet $V$ de $\operatorname{Rep}_{\mathfrak{C}}^{\mathrm{WT}}\left(\pi_{1}(C, \bar{x})\right)$, quitte à remplacer $S$ par une extension finie, il existe un $S$-modèle semi-stable et régulier $X$ de $C$ tel que $V$ soit de Weil-Tate relativement à $X$. D'après (10.12.4), on a un isomorphisme

$$
V \stackrel{\sim}{\rightarrow} \mathscr{V}_{\check{C}}\left(\mathscr{T}_{\check{C}}(V)\right)
$$

Il résulte de la fonctorialité de (10.12.4) et de 10.17(ii) que celui-là est fonctoriel en $V$.

De même, pour tout objet $F$ de $\mathfrak{V}_{\check{C}}$, quitte à remplacer $S$ par une extension finie, il existe un $S$-modèle semi-stable et régulier $X$ de $C$ et un objet $\mathscr{F}$ de $\operatorname{Mod}^{\mathrm{WT}}\left(\mathscr{O}_{\mathfrak{X}}\left[\frac{1}{p}\right]\right)$ tels que $F \simeq J \mathfrak{X}(\mathscr{F})$. D'après $(10.12 .2)$, on a un isomorphisme

$$
F \stackrel{\sim}{\rightarrow} \mathscr{T}_{\check{C}}\left(\mathscr{V}_{\check{C}}(F)\right)
$$

Il résulte de la fonctorialité de (10.12.2) et de 10.17(i) que celui-là est fonctoriel en $F$.

THÉORÈME 10.20. Tout fibré vectoriel de Deninger-Werner sur $\check{C}$ est de WeilTate. De plus, la restriction du foncteur $\mathscr{V}_{\check{C}}: \mathfrak{V}_{\check{C}}^{\mathrm{WT}} \rightarrow \operatorname{Rep}_{\mathfrak{C}}^{\mathrm{cont}}\left(\pi_{1}(C, \bar{x})\right)(10.18 .1)$ à la sous-catégorie $\mathfrak{V}_{\check{C}}^{\mathrm{DW}}$ s'identifie au foncteur de Deninger-Werner $\mathbb{V}_{\check{C}}$ (6.14.1).

On présentera la démonstration dans 14.5 et 14.6.

COROLlaire 10.21. Le foncteur de Deninger-Werner $\mathbb{V}_{\check{C}}$ (6.14.1) est pleinement fidèle.

Preuve. Cela résulte de 10.19 et 10.20 .

Proposition 10.22. Soit $\pi: C^{\prime} \rightarrow C$ un revêtement étale connexe, $\bar{x}^{\prime}$ un $\bar{\eta}$-point de $C^{\prime}$ au-dessus de $\bar{x}, \check{C}^{\prime}=C^{\prime} \otimes_{\bar{K}} \mathfrak{C}$ et $\check{\pi}=\pi \otimes_{\bar{K}} \mathfrak{C}$. Alors : 
(i) l'image inverse d'un fibré vectoriel de Weil-Tate sur $\check{C}$ par $\check{\pi}$ est de Weil-Tate sur $\check{C}^{\prime}$;

(ii) toute $\mathfrak{C}$-représentation continue de Weil-Tate de $\pi_{1}(C, \bar{x})$ définit par restriction (10.13.3) une $\mathfrak{C}$-représentation continue de Weil-Tate de $\pi_{1}\left(C^{\prime}\right.$, $\left.\bar{x}^{\prime}\right)$;

(iii) le morphisme $\pi$ induit des diagrammes commutatifs à isomorphismes près

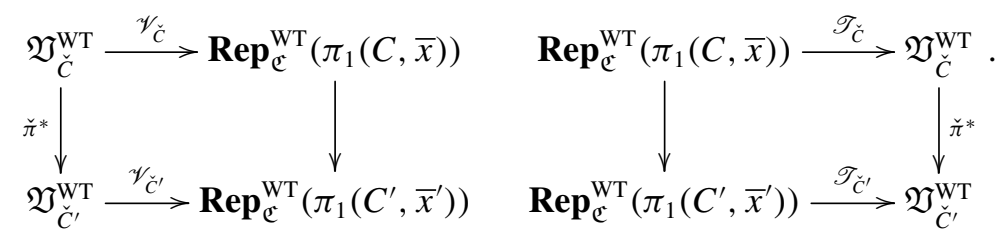

Cela résulte de 10.14.

\section{Correspondance de Simpson $p$-adique pour les représentations de Weil-Tate}

11.1. On munit $S$ de la structure logarithmique $\mathscr{M}_{S}$ définie par son point fermé [4, II.6.1], autrement dit $\mathscr{M}_{S}=j_{*}\left(\mathscr{O}_{\eta}^{\times}\right) \cap \mathscr{O}_{S}$, où $j: \eta \rightarrow S$ est l'injection canonique. On munit $\bar{S}$ et $\check{\bar{S}}(2.1)$ des structures logarithmiques $\mathscr{M}_{\bar{S}}$ et $\mathscr{M}_{\check{\bar{S}}}$, images inverses de $\mathscr{M}_{S}$ (cf. [4, II.5.10]).

On associe fonctoriellement à toute $\mathbb{Z}_{(p)}$-algèbre $A$ l'anneau (cf. [4, II.9.3])

$$
A^{b}=\lim _{x \mapsto x^{p}} A / p A
$$

et un homomorphisme $\theta: W\left(A^{b}\right) \rightarrow \widehat{A}$ de l'anneau $W\left(A^{b}\right)$ des vecteurs de Witt de $A^{\text {b }}$ dans le séparé complété $p$-adique $\widehat{A}$ de $A$.

On désigne par $\underline{p}$ l'élément de $\left(\mathcal{O}_{\bar{K}}\right)^{\mathrm{b}}$ induit par la suite $\left(p^{\frac{1}{p n}}\right)_{n \geqslant 0}(2.1)$ et l'on pose

$$
\xi=[\underline{p}]-p \in W\left(\left(\mathcal{O}_{\bar{K}}\right)^{b}\right),
$$

où [ ] est le représentant multiplicatif. On pose

$$
\mathscr{A}_{2}\left(\mathcal{O}_{\bar{K}}\right)=W\left(\left(\mathcal{O}_{\bar{K}}\right)^{b}\right) / \operatorname{Ker}(\theta)^{2} .
$$

On a une suite exacte [4, II.9.5(iv)]

$$
0 \longrightarrow W\left(\left(\mathcal{O}_{\bar{K}}\right)^{b}\right) \stackrel{\cdot \xi}{\longrightarrow} W\left(\left(\mathcal{O}_{\bar{K}}\right)^{b}\right) \stackrel{\theta}{\longrightarrow} \mathfrak{o} \longrightarrow 0 .
$$


Elle induit une suite exacte

$$
0 \longrightarrow \mathfrak{o} \stackrel{\xi}{\longrightarrow} \mathscr{A}_{2}\left(\mathcal{O}_{\bar{K}}\right) \stackrel{\theta}{\longrightarrow} \mathfrak{o} \longrightarrow 0,
$$

où $\cdot \xi$ est induit par la multiplication par $\xi$ dans $\mathscr{A}_{2}\left(\mathcal{O}_{\bar{K}}\right)$. L'idéal $\operatorname{Ker}(\theta)$ est un $\mathfrak{o}$ module libre de base $\xi$. On le note $\xi \mathfrak{o}$ et on note $\xi^{-1} \mathfrak{o}$ le $\mathfrak{o}$-module dual de $\xi \mathfrak{o}$. Pour tout $\mathfrak{o}$-module $M$, on désigne les $\mathfrak{o}$-modules $M \otimes_{\mathfrak{o}} \xi \mathfrak{o}$ et $M \otimes_{\mathfrak{o}} \xi^{-1} \mathfrak{o}$ simplement par $\xi M$ et $\xi^{-1} M$ respectivement.

On pose

$$
\mathscr{A}_{2}(\bar{S})=\operatorname{Spec}\left(\mathscr{A}_{2}\left(\mathcal{O}_{\bar{K}}\right)\right)
$$

que l'on munit de la structure logarithmique $\mathscr{M}_{\mathscr{A}_{2}(\bar{S})}$ définie dans [4, II.9.8]. L'homomorphisme $\theta$ induit alors une immersion fermée exacte [4, II.5.22]

$$
i_{\bar{S}}:\left(\check{\bar{S}}, \mathscr{M}_{\overline{\bar{S}}}\right) \rightarrow\left(\mathscr{A}_{2}(\bar{S}), \mathscr{M}_{\mathscr{A}_{2}(\bar{S})}\right)
$$

11.2. Dans la suite de cette section, on se donne une $S$-courbe semi-stable et régulière $X$ que l'on munit de la structure logarithmique $\mathscr{M}_{X}$ définie par sa fibre spéciale $X_{s}$. Le morphisme de schémas logarithmiques $f:\left(X, \mathscr{M}_{X}\right) \rightarrow\left(S, \mathscr{M}_{S}\right)$ est alors adéquat au sens de [4, III.4.7]. On notera que $X_{\eta}$ est le sous-schéma ouvert maximal de $X$ où la structure logarithmique $\mathscr{M}_{X}$ est triviale. Pour alléger les notations, on pose

$$
\widetilde{\Omega}_{X / S}^{1}=\Omega_{\left(X, \mathscr{M}_{X}\right) /\left(S, \mathscr{M}_{S}\right)}^{1} .
$$

On munit $\check{\bar{X}}=X \times{ }_{S} \check{\bar{S}}(2.1 .1)$ de la structure logarithmique $\mathscr{M}_{\overline{\mathrm{X}}}$, image inverse de $\mathscr{M}_{X}$. Posons $\mathscr{T}_{X / S}=\mathscr{H}_{0 m_{\mathscr{O}_{X}}}\left(\widetilde{\Omega}_{X / S}^{1}, \mathscr{O}_{X}\right)$ et $\mathscr{T}_{\overline{\bar{X}} / \overline{\bar{S}}}=\mathscr{T}_{X / S} \otimes_{\mathscr{O}_{S}} \mathscr{O}_{\overline{\bar{S}}}$. Comme $X$ est une $S$-courbe, on a alors $\mathrm{H}^{2}\left(X_{\eta}, \mathscr{T}_{X / S, \eta}\right)=0$ et $\mathrm{H}^{2}\left(X_{s}, \mathscr{T}_{X / S, s}\right)=0$. Compte tenu de [35, Section 5, Cor. 2], $\mathrm{R}^{2} f_{*}\left(\mathscr{T}_{X / S}\right)$ est localement libre de type fini. En outre, on a $\mathrm{R}^{2} f_{*}\left(\mathscr{T}_{X / S}\right)_{\eta}=\mathrm{H}^{2}\left(X_{\eta}, \mathscr{T}_{X / S, \eta}\right)=0$. On en déduit que $\mathrm{H}^{2}\left(X, \mathscr{T}_{X / S}\right)=$ 0 et $\mathrm{H}^{2}\left(\check{\bar{X}}, \mathscr{T}_{\overline{\bar{X}} / \bar{S}}\right)=0$. D'après [29, Prop. 3.14], il existe une $\left(\mathscr{A}_{2}(\bar{S}), \mathscr{M}_{\mathscr{A}_{2}(\bar{S})}\right)$ déformation lisse $\left(\tilde{X}, \mathscr{M}_{\tilde{X}}\right)$ de $\left(\check{\bar{X}}, \mathscr{M}_{\overline{\bar{X}}}\right)$, c'est-à-dire un diagramme cartésien

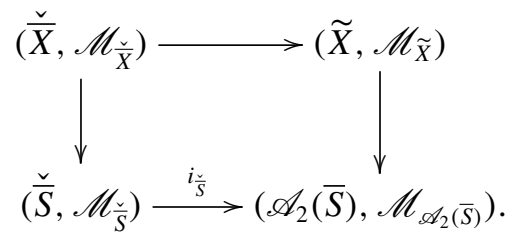

On fixe une telle déformation $\left(\tilde{X}, \mathscr{M}_{\tilde{X}}\right)$ dans la suite de cette section. 
11.3. On note $\mathfrak{X}$ le schéma formel complété $p$-adique de $\bar{X}$. On désigne par $\xi^{-1} \widetilde{\Omega}_{\mathfrak{X} / \mathscr{S}}^{1}$ le complété $p$-adique du $\mathscr{O}_{\bar{X}}$-module $\xi^{-1} \widetilde{\Omega}_{\bar{X} / \bar{S}}^{1}=\xi^{-1} \widetilde{\Omega}_{X / S}^{1} \otimes_{\mathscr{O}_{X}} \mathscr{O}_{\bar{X}}$ et on pose, pour tout entier $j \geqslant 0, \xi^{-j} \widetilde{\Omega}_{\mathfrak{X} / \mathscr{S}}^{j}=\wedge_{\mathscr{O}_{\mathfrak{X}}}^{j}\left(\xi^{-1} \widetilde{\Omega}_{\mathfrak{X} / \mathscr{S}}^{1}\right)$. On sous-entend par $\mathscr{O}_{\mathfrak{X}}\left[\frac{1}{p}\right]$-module de Higgs à coefficients dans $\xi^{-1} \widetilde{\Omega}_{\mathfrak{X} / \mathscr{S}}^{1}$ un $\mathscr{O}_{\mathfrak{X}}\left[\frac{1}{p}\right]$-module de Higgs à coefficients dans $\xi^{-1} \widetilde{\Omega}_{\mathfrak{X} / \mathscr{S}}^{1}\left[\frac{1}{p}\right]$ (2.10). On désigne par $\mathbf{M H}\left(\mathscr{O}_{\mathfrak{X}}\left[\frac{1}{p}\right]\right.$, $\left.\xi^{-1} \widetilde{\Omega}_{\mathfrak{X} / \mathscr{S}}^{1}\right)$ la catégorie de tels modules et par $\mathbf{M} \mathbf{H}^{\mathrm{coh}}\left(\mathscr{O}_{\mathfrak{X}}\left[\frac{1}{p}\right], \xi^{-1} \widetilde{\Omega}_{\mathfrak{X} / \mathscr{S}}^{1}\right)$ la souscatégorie pleine formée des modules de Higgs dont le $\mathscr{O}_{\mathfrak{X}}\left[\frac{1}{p}\right]$-module sous-jacent est cohérent. On appelle $\mathscr{O}_{\mathfrak{X}}\left[\frac{1}{p}\right]$-fibré de Higgs à coefficients dans $\xi^{-1} \widetilde{\Omega}_{\mathfrak{X} / \mathscr{S}}^{1}$ tout $\mathscr{O}_{\mathfrak{X}}\left[\frac{1}{p}\right]$-module de Higgs à coefficients dans $\xi^{-1} \widetilde{\Omega}_{\mathfrak{X} / \mathscr{S}}^{1}$ dont le $\mathscr{O}_{\mathfrak{X}}\left[\frac{1}{p}\right]$-module sous-jacent est localement projectif de type fini (2.9).

11.4. On reprend les notations de la Section 7 pour le $S$-schéma $X$. Soit $\mathscr{M}$ un $\overline{\mathscr{B}}_{\mathbb{Q}^{-}}$-module adique et de type fini (7.15) et $\mathscr{N}$ un $\mathscr{O}_{\mathfrak{X}}\left[\frac{1}{p}\right]$-fibré de Higgs à coefficients dans $\xi^{-1} \widetilde{\Omega}_{\mathfrak{X} / \mathscr{S}}^{1}$. Dans [4, III.12.10(ii)], Abbes et Gros définissent la propriété selon laquelle $\mathscr{M}$ et $\mathscr{N}$ sont associés. On dit que $\mathscr{M}$ est de Dolbeault s'il admet un fibré de Higgs associé et que $\mathscr{N}$ est soluble s'il admet un $\breve{\mathscr{B}}_{\mathbb{Q}^{-}}$ module associé (cf. [4, III.12.11]).

On désigne par $\operatorname{Mod}_{\mathbb{Q}}^{\text {Dolb }}(\stackrel{\breve{\mathscr{B}}}{)})$ la sous-catégorie pleine de $\operatorname{Mod}_{\mathbb{Q}}^{\text {atf }}(\breve{\mathscr{B}})$ (7.15) formée des $\breve{\mathscr{B}}_{\mathbb{Q}}$-modules de Dolbeault et par $\mathbf{M H}^{\text {sol }}\left(\mathscr{O}_{\mathfrak{X}}\left[\frac{1}{p}\right], \xi^{-1} \widetilde{\Omega}_{\mathfrak{X} / \mathscr{S}}^{1}\right)$ la souscatégorie pleine de $\mathbf{M H}\left(\mathscr{O}_{\mathfrak{X}}\left[\frac{1}{p}\right], \xi^{-1} \widetilde{\Omega}_{\mathfrak{X} / \mathscr{S}}^{1}\right)$ formée des $\mathscr{O}_{\mathfrak{X}}\left[\frac{1}{p}\right]$-fibrés de Higgs solubles à coefficients dans $\xi^{-1} \widetilde{\Omega}_{\mathfrak{X} / \mathscr{S}}^{1}$. En vertu de [4, III.12.18], il existe un foncteur

$$
\mathscr{H}: \operatorname{Mod}_{\mathbb{Q}}^{\text {Dolb }(\breve{\mathscr{B}})} \rightarrow \mathbf{M H}^{\mathrm{sol}}\left(\mathscr{O}_{\mathfrak{X}}\left[\frac{1}{p}\right], \xi^{-1} \widetilde{\Omega}_{\mathfrak{X} / \mathscr{S}}^{1}\right) .
$$

D'après [4, III.12.23], il existe un foncteur dans l'autre sens

$$
\mathscr{W}: \mathbf{M H}^{\mathrm{sol}}\left(\mathscr{O}_{\mathfrak{X}}\left[\frac{1}{p}\right], \xi^{-1} \widetilde{\Omega}_{\mathfrak{X} / \mathscr{S}}^{1}\right) \rightarrow \operatorname{Mod}_{\mathbb{Q}}^{\mathrm{Dolb}}(\overline{\mathscr{\mathscr { B }}}) .
$$

THÉORÈme 11.5 [4, III.12.26]. Les foncteurs (11.4.1) et (11.4.2) sont des équivalences de catégories quasi inverses l'une de l'autre.

LEMME 11.6. Soit $\mathscr{F}$ un $\mathscr{O}_{\mathfrak{X}}\left[\frac{1}{p}\right]$-module localement projectif de type fini. Alors, le $\breve{\mathscr{B}}_{\mathbb{Q}}$-module $\mathrm{T}^{*}(\mathscr{F})$ (10.1.4) et le fibré de Higgs $(\mathscr{F}, 0)$ sont associés (11.4) et l'on a un isomorphisme canonique et fonctoriel

$$
(\mathscr{F}, 0) \stackrel{\sim}{\rightarrow} \mathscr{H}\left(\mathrm{T}^{*}(\mathscr{F})\right) .
$$


Preuve. Démontrons que $\mathrm{T}^{*}(\mathscr{F})$ et $(\mathscr{F}, 0)$ sont $r$-associés dans le sens de [4, III.12.10(i)] pour tout nombre rationnel $r>0$. On désigne par $\breve{\mathscr{C}}^{(r)}$ la $\breve{\mathscr{B}}$-algèbre de Higgs-Tate d'épaisseur r associée à $\left(\widetilde{X}, \mathscr{M}_{\tilde{X}}\right)$ (cf. [4, III.10.31]), par $\breve{d}^{(r)}$ la $\breve{\mathscr{B}}$ dérivation universelle de $\breve{\mathscr{C}}^{(r)}$ définie dans [4, (III.12.7.1)], par $\Xi^{r}$ la catégorie des $p^{r}$-isoconnexions intégrables relativement à l'extension $\breve{\mathscr{C}}^{(r)} / \breve{\mathscr{B}}$ (cf. [4, III.6.10]) et par $\Xi_{\mathbb{Q}}^{r}$ la catégorie des objets de $\Xi^{r}$ à isogénie près (2.16). Dans la suite, on va construire un isomorphisme canonique fonctoriel

$$
\mathrm{T}^{r+}(\mathscr{F}, 0) \stackrel{\sim}{\rightarrow} \mathfrak{S}^{r}\left(\mathrm{~T}^{*}(\mathscr{F})\right),
$$

où $\mathfrak{S}^{r}: \operatorname{Mod}_{\mathbb{Q}}^{\text {atf }}(\breve{\mathscr{B}}) \rightarrow \Xi_{\mathbb{Q}}^{r}$ et $\mathrm{T}^{r+}: \mathbf{M H}^{\text {coh }}\left(\mathscr{O}_{\mathfrak{X}}\left[\frac{1}{p}\right], \xi^{-1} \widetilde{\Omega}_{\mathfrak{X} / \mathscr{S}}^{1}\right) \rightarrow \Xi_{\mathbb{Q}}^{r}$ sont des foncteurs [4, (III.12.7.3) et (III.12.7.8)]. En vertu de (4.1.2), il existe un $\mathscr{O}_{\mathfrak{X}^{-}}$ module cohérent $\mathscr{G}$ tel que $\mathscr{F} \simeq \mathscr{G}\left[\frac{1}{p}\right]$. Par définition de $\mathfrak{S}^{r}$ [4, (III.12.7.2)], on a

$$
\mathfrak{S}^{r}\left(\mathrm{~T}^{*}(\mathscr{F})\right)=\left(\breve{\mathscr{C}}^{(r)} \otimes_{\breve{\mathscr{B}}} \mathrm{T}^{*}(\mathscr{G}), \breve{\mathscr{C}}^{(r)} \otimes_{\breve{\mathscr{B}}} \mathrm{T}^{*}(\mathscr{G}), \mathrm{id}, p^{r} \breve{d}^{(r)} \otimes \mathrm{id}\right)_{\mathbb{Q}} .
$$

Compte tenu de la définition de $\mathrm{T}^{r+}$ [4, (III.12.7.6)], on a $\mathrm{T}^{r+}(\mathscr{F}, 0)=\left(\breve{\mathscr{C}}^{(r)} \otimes_{\breve{\mathscr{B}}} \mathrm{T}^{*}(\mathscr{G}), \breve{\mathscr{C}}^{(r)} \otimes_{\breve{\mathscr{B}}} \mathrm{T}^{*}(\mathscr{G}), \mathrm{id} \otimes_{\frac{\breve{\mathscr{B}}}{}} \mathrm{T}^{*}(\mathrm{id}), p^{r} \breve{d}^{(r)} \otimes \mathrm{T}^{*}(\mathrm{id})\right)_{\mathbb{Q}}$.

On prend pour (11.6.2) l'isomorphisme induit par (11.6.3) et (11.6.4). D'après [4, III.12.17(i)], on en déduit un isomorphisme

$$
(\mathscr{F}, 0) \stackrel{\sim}{\rightarrow} \mathscr{H}\left(\mathrm{T}^{*}(\mathscr{F})\right) .
$$

La fonctorialité de (11.6.1) se déduit de celle de (11.6.2). Le lemme s’ensuit.

Proposition 11.7. Soit $\bar{x}$ un point géométrique de $X_{\bar{\eta}}$ et $V$ une $\mathfrak{C}$ représentation continue de $\pi_{1}\left(X_{\bar{\eta}}, \bar{x}\right)$ (3.18). Alors, les conditions suivantes sont équivalentes:

(a) Le $\breve{\mathscr{B}}_{\mathbb{Q}}$-module $\breve{\beta}_{\mathbb{Q}}^{*}(V)$ (8.6.3) est de Dolbeault (11.4) et le fibré de Higgs $\mathscr{H}\left(\breve{\beta}_{\mathbb{Q}}^{*}(V)\right)$ est de champ de Higgs nul.

(b) $V$ est de Weil-Tate relativement à $X$ (10.3).

De plus, si les conditions sont remplies, on a un isomorphisme canonique fonctoriel de fibrés de Higgs

$$
\mathscr{H}\left(\breve{\beta}_{\mathbb{Q}}^{*}(V)\right) \stackrel{\sim}{\rightarrow}\left(\mathscr{T}_{\mathfrak{X}}(V), 0\right),
$$

où $\mathscr{T}_{\mathfrak{X}}$ est le foncteur (10.11.1). 
Preuve. Supposons d'abord que la condition (a) soit remplie. On note $\mathscr{F}$ le $\mathscr{O}_{\mathfrak{X}}\left[\frac{1}{p}\right]$-module localement projectif de type fini sous-jacent à $\mathscr{H}\left(\breve{\beta}_{\mathbb{Q}}^{*}(V)\right)$. D'après 11.6, on a un isomorphisme canonique

$$
(\mathscr{F}, 0) \stackrel{\sim}{\rightarrow} \mathscr{H}\left(\mathrm{T}^{*}(\mathscr{F})\right) .
$$

En vertu de 11.5, on en déduit un isomorphisme de $\breve{\mathscr{B}}_{\mathbb{Q}}$-modules

$$
\breve{\beta}_{\mathbb{Q}}^{*}(V) \stackrel{\sim}{\rightarrow} \mathrm{T}^{*}(\mathscr{F})
$$

d'où la condition (b).

Supposons que la condition (b) soit vraie. D'après 10.10 , le $\mathscr{O}_{\mathfrak{X}}\left[\frac{1}{p}\right]$-module $\mathscr{T}_{\mathfrak{X}}(V)$ est localement projectif de type fini. De plus, on a un isomorphisme fonctoriel en $V(10.10 .1)$

$$
\breve{\beta}_{\mathbb{Q}}^{*}(V) \stackrel{\sim}{\rightarrow} \mathrm{T}^{*}\left(\mathscr{T}_{\mathfrak{X}}(V)\right) .
$$

La condition (a) et l'isomorphisme (11.7.1) résultent alors de 11.6 appliqué à $\mathscr{F}=\mathscr{T}_{\mathfrak{X}}(V)$.

Proposition 11.8. Soit $C$ une $\bar{K}$-courbe propre et lisse, $\bar{x}$ un point géométrique de $C, V$ une $\mathfrak{C}$-représentation de Weil-Tate de $\pi_{1}(C, \bar{x})$ et $\mathscr{T}(V)$ le fibré vectoriel sur $\check{C}=C \otimes_{\bar{K}} \mathfrak{C}$ associé à $V$. On a alors une suite exacte

$$
\begin{aligned}
0 & \rightarrow \mathrm{H}^{1}\left(\check{C}_{\mathrm{zar}}, \mathscr{T}(V)\right) \rightarrow \mathrm{H}_{\mathrm{cont}}^{1}\left(\pi_{1}(C, \bar{x}), V\right) \\
& \rightarrow \mathrm{H}^{0}\left(\check{C}_{\mathrm{zar}}, \mathscr{T}(V) \otimes_{\mathscr{O}_{\check{C}}}\left(\xi^{-1} \Omega_{\check{C} / \mathfrak{C}}^{1}\right)\right) \rightarrow 0,
\end{aligned}
$$

où $\mathrm{H}_{\mathrm{cont}}^{1}\left(\pi_{1}(C, \bar{x}),-\right)$ désigne le groupe de cohomologie continue de $\pi_{1}(C, \bar{x})$ (3.30).

Preuve. Quitte à remplacer $K$ par une extension finie, il existe un $S$-modèle semistable et régulier $X$ de $C$ tel que $V$ soit de Weil-Tate relativement à $X$ (10.3). Reprenant les notations précédentes pour $X$, on a un isomorphisme canonique de $\breve{\mathscr{B}}_{\mathbb{Q}}$-modules

$$
\breve{\beta}_{\mathbb{Q}}^{*}(V) \simeq \mathrm{T}^{*}\left(\mathscr{T}_{\mathfrak{X}}(V)\right)
$$

Reprenant les notations de 2.17 pour le topos annelé $\left(\widetilde{E}_{s}^{\mathbb{N}^{\circ}}, \breve{\mathscr{B}}\right)$, d'après $(3.30)$ et (7.21), le foncteur $\breve{\beta}_{\mathbb{Q}}^{*}(8.6 .3)$ induit un isomorphisme canonique

$$
\mathrm{H}_{\text {cont }}^{1}\left(\pi_{1}(C, \bar{x}), V\right) \stackrel{\sim}{\rightarrow} \mathrm{H}^{1}\left(\widetilde{E}_{s}^{\mathbb{N}^{\circ}}, \breve{\beta}_{\mathbb{Q}}^{*}(V)\right) .
$$


Par ailleurs, choisissant un $\mathscr{O}_{\mathfrak{X}}$-module cohérent $\mathscr{F}$ tel que $\mathscr{F}\left[\frac{1}{p}\right] \simeq \mathscr{T}_{\mathfrak{X}}(V)$ (4.1.2), on a une suite exacte

$$
\mathrm{H}^{i}\left(\mathfrak{X}, \mathrm{R}^{j} \mathrm{~T}_{*}\left(\mathrm{~T}^{*}(\mathscr{F})\right)\right) \Rightarrow \mathrm{H}^{i+j}\left(\widetilde{E}_{s}^{\mathbb{N}^{\circ}}, \mathrm{T}^{*}(\mathscr{F})\right) .
$$

Compte tenu de 5.16(i), on en déduit une suite spectrale

$$
\mathrm{H}^{i}\left(\mathfrak{X}, \mathrm{R}^{j} \mathrm{~T}_{*}\left(\mathrm{~T}^{*}\left(\mathscr{T}_{\mathfrak{X}}(V)\right)\right)\right) \Rightarrow \mathrm{H}^{i+j}\left(\widetilde{E}_{s}^{\mathbb{N}^{\circ}}, \mathrm{T}^{*}\left(\mathscr{T}_{\mathfrak{X}}(V)\right)\right) .
$$

D'après $(11.7 .1)$ et $\left[4\right.$, III.12.34], on a un isomorphisme canonique de $\mathscr{O}_{\mathfrak{X}}\left[\frac{1}{p}\right]$ modules

$$
\mathrm{R}^{j} \mathrm{~T}_{*}\left(\mathrm{~T}^{*}\left(\mathscr{T}_{\mathfrak{X}}(V)\right)\right) \simeq \mathscr{T}_{\mathfrak{X}}(V) \otimes_{\mathscr{O}_{\mathfrak{X}}} \xi^{-j} \widetilde{\Omega}_{\mathfrak{X} / \mathscr{S}}^{j} .
$$

Compte tenu de 5.16(ii), on a un isomorphisme $\mathfrak{C}$-linéaire

$$
\mathrm{H}^{i}\left(\mathfrak{X}, \mathscr{T}_{\mathfrak{X}}(V) \otimes_{\mathscr{O}_{\mathfrak{X}}}\left(\xi^{-j} \widetilde{\Omega}_{\mathfrak{X} / \mathscr{S}}^{j}\right)\right) \simeq \mathrm{H}^{i}\left(\check{C}_{\mathrm{zar}}, \mathscr{T}(V) \otimes_{\mathscr{O}_{\check{C}}}\left(\xi^{-j} \Omega_{\check{C} / \mathfrak{C}}^{j}\right)\right) .
$$

Comme $C$ est une courbe sur $\bar{K}$, ce dernier est nul si $i \geqslant 2$. On en déduit par (11.8.4) une suite exacte de $\mathfrak{C}$-espaces vectoriels

$$
\begin{aligned}
0 & \rightarrow \mathrm{H}^{1}\left(\check{C}_{\text {zar }}, \mathscr{T}(V)\right) \rightarrow \mathrm{H}^{1}\left(\widetilde{E}_{s}^{\mathbb{N}^{\circ}}, \mathrm{T}^{*}\left(\mathscr{T}_{\mathfrak{X}}(V)\right)\right) \\
& \rightarrow \mathrm{H}^{0}\left(\check{C}_{\text {zar }}, \mathscr{T}(V) \otimes_{\mathscr{O}_{\check{C}}}\left(\xi^{-1} \Omega_{\check{C} / \mathbb{C}^{\mathfrak{C}}}^{1}\right)\right) \rightarrow 0 .
\end{aligned}
$$

La proposition s'ensuit compte tenu de (11.8.2), (11.8.3) et (11.8.5).

\section{Faisceaux de $\alpha$-modules}

12.1. Pour toute $\mathfrak{o}$-algèbre $A$, on désigne par $\operatorname{Mod}(A)$ la catégorie abélienne tensorielle des $A$-modules. Suivant 2.18, prenant pour $\phi: \mathfrak{o} \rightarrow A=\operatorname{End}\left(\operatorname{id}_{\operatorname{Mod}(A)}\right)$ l'homomorphisme structural, on appelle catégorie des $\alpha$-A-modules et l'on note $\alpha-\operatorname{Mod}(A)$ le quotient de la catégorie $\operatorname{Mod}(A)$ par la sous-catégorie épaisse des $A$-modules $\alpha$-nuls. On désigne par

$$
\alpha: \operatorname{Mod}(A) \rightarrow \alpha-\operatorname{Mod}(A), \quad M \mapsto M^{\alpha},
$$

le foncteur canonique (2.18.1). Pour tous $A$-modules $M$ et $N$, on a un isomorphisme canonique fonctoriel [3, (1.4.7.1)]

$$
\operatorname{Hom}_{\alpha-\operatorname{Mod}(A)}\left(M^{\alpha}, N^{\alpha}\right) \stackrel{\sim}{\rightarrow} \operatorname{Hom}_{\operatorname{Mod}(A)}\left(\mathfrak{m} \otimes_{\mathfrak{o}} M, N\right) .
$$

On désigne par $\sigma_{*}$ le foncteur

$$
\sigma_{*}: \alpha-\operatorname{Mod}(A) \rightarrow \operatorname{Mod}(A), \quad P \mapsto \operatorname{Hom}_{\alpha-\operatorname{Mod}(A)}\left(A^{\alpha}, P\right),
$$


et par $\sigma_{!}$le foncteur

$$
\sigma_{!}: \alpha-\operatorname{Mod}(A) \rightarrow \operatorname{Mod}(A), \quad P \mapsto \mathfrak{m} \otimes_{\mathfrak{o}} \sigma_{*}(P) .
$$

En vertu de (12.1.2), pour tout $A$-module $M$, on a un isomorphisme canonique fonctoriel

$$
\sigma_{*}\left(M^{\alpha}\right) \stackrel{\sim}{\rightarrow} \operatorname{Hom}_{\mathfrak{o}}(\mathfrak{m}, M) .
$$

Proposition $12.2[3,1.4 .8]$. (i) Le foncteur $\sigma_{*}$ est un adjoint à droite du foncteur $\alpha$ (12.1.1).

(ii) Le morphisme d'adjonction $\alpha \circ \sigma_{*} \rightarrow \mathrm{id}$ est un isomorphisme.

(iii) Le foncteur $\sigma_{!}$est un adjoint à gauche du foncteur $\alpha$.

(iv) Le morphisme d'adjonction id $\rightarrow \alpha \circ \sigma_{!}$est un isomorphisme.

Corollaire 12.3 [3, 1.4.9]. Les foncteurs $\alpha$ et $\sigma_{!}$sont exacts et le foncteur $\sigma_{*}$ est exact à gauche.

12.4. On appelle $\alpha$-o-algèbre (ou $\mathfrak{o}^{\alpha}$-algèbre) un monoïde unitaire commutatif de $\alpha-\operatorname{Mod}(\mathfrak{o})$ [3, 1.4.11]. On désigne par $\operatorname{Alg}(\mathfrak{o})$ la catégorie des o-algèbres et par $\alpha$-Alg $(\mathfrak{o})$ la catégorie des $\alpha$-o-algèbres. Le foncteur $\alpha$ étant monoïdal, il induit un foncteur que l'on note encore

$$
\alpha: \operatorname{Alg}(\mathfrak{o}) \rightarrow \alpha-\operatorname{Alg}(\mathfrak{o}) .
$$

Compte tenu de l'isomorphisme canonique $A^{\alpha} \stackrel{\sim}{\rightarrow} A^{\alpha} \otimes_{A^{\alpha}} A^{\alpha}$, le foncteur $\sigma_{*}$ (12.1.3) induit un foncteur que l'on note encore

$$
\sigma_{*}: \alpha-\operatorname{Alg}(\mathfrak{o}) \rightarrow \operatorname{Alg}(\mathfrak{o}), \quad P \mapsto \operatorname{Hom}_{\alpha-\operatorname{Mod}(A)}\left(A^{\alpha}, P\right) .
$$

En vertu de [3, 1.4.12], celui-ci est un adjoint à droite du foncteur $\alpha$ (12.4.1) et le morphisme d'adjonction $\alpha \circ \sigma_{*} \rightarrow$ id est un isomorphisme.

12.5. Soit $A$ une o-algèbre. On pose $A^{\alpha}=\alpha(A)$ (12.4.1) et on désigne par $\operatorname{Mod}\left(A^{\alpha}\right)$ la catégorie des $A^{\alpha}$-modules unitaires de $\alpha-\operatorname{Mod}(\mathfrak{o})$. Le foncteur $\alpha$ : $\operatorname{Mod}(\mathfrak{o}) \rightarrow \alpha-\operatorname{Mod}(\mathfrak{o})$ étant monoïdal, il induit un foncteur

$$
\alpha_{A}: \operatorname{Mod}(A) \rightarrow \operatorname{Mod}\left(A^{\alpha}\right) .
$$

Celui-ci transforme les $\alpha$-isomorphismes en des isomorphismes. Il induit une équivalence de catégories [3, 1.4.13]

$$
\alpha-\operatorname{Mod}(A) \stackrel{\sim}{\rightarrow} \operatorname{Mod}\left(A^{\alpha}\right) .
$$


On pose $A^{\prime}=\sigma_{*}\left(A^{\alpha}\right)$ (12.4.2). D'après 12.4 , on a un morphisme canonique de o-algèbres $\lambda: A \rightarrow A^{\prime}$ qui induit un isomorphisme $A^{\alpha} \stackrel{\sim}{\rightarrow} A^{\prime \alpha}$. Pour tous $\mathfrak{o}^{\alpha}$-modules $P$ et $Q$, on a un morphisme o-linéaire canonique

$$
\operatorname{Hom}_{\alpha-\operatorname{Mod}(\mathfrak{o})}\left(\mathfrak{o}^{\alpha}, P\right) \otimes_{\mathfrak{o}} \operatorname{Hom}_{\alpha-\operatorname{Mod}(\mathfrak{o})}\left(\mathfrak{o}^{\alpha}, Q\right) \rightarrow \operatorname{Hom}_{\alpha-\operatorname{Mod}(\mathfrak{o})}\left(\mathfrak{o}^{\alpha}, P \otimes_{\mathfrak{o}^{\alpha}} Q\right)
$$

défini par fonctorialité et composition. Par suite, le foncteur $\sigma_{*}(12.1 .3)$ induit un foncteur

$$
\tau_{*}^{\prime}: \operatorname{Mod}\left(A^{\alpha}\right) \rightarrow \operatorname{Mod}\left(A^{\prime}\right) .
$$

Composant avec le foncteur induit par $\lambda: A \rightarrow A^{\prime}$, on obtient un foncteur

$$
\tau_{*}: \operatorname{Mod}\left(A^{\alpha}\right) \rightarrow \operatorname{Mod}(A) .
$$

On désigne par $\tau_{!}$le foncteur

$$
\tau_{!}: \operatorname{Mod}\left(A^{\alpha}\right) \rightarrow \operatorname{Mod}(A), \quad P \mapsto \mathfrak{m} \otimes_{\mathfrak{o}} \tau_{*}(P) .
$$

Le foncteur $\tau_{*}$ (resp. $\tau_{!}$) est un adjoint à droite (resp. à gauche) de $\alpha_{A}$ et les morphismes d'adjonction $\alpha_{A} \circ \tau_{*} \rightarrow$ id et id $\rightarrow \alpha_{A} \circ \tau_{\text {! }}$ sont des isomorphismes (cf. [3, 1.4.13]).

12.6. Dans la suite de cette section, $\mathscr{C}$ désigne un site, $\widehat{\mathscr{C}}$ la catégorie des préfaisceaux d'ensembles sur $\mathscr{C}$ et $\widetilde{\mathscr{C}}$ le topos des faisceaux d'ensembles sur $\mathscr{C}$. On note $\mathfrak{o}_{\widehat{\mathscr{C}}}\left(\right.$ resp. $\left.\mathfrak{m}_{\widehat{\mathscr{C}}}\right)$ le préfaisceau constant sur $\mathscr{C}$ de valeur $\mathfrak{o}$ (resp. m) et $\mathfrak{o}_{\mathscr{C}}\left(\operatorname{resp} . \mathfrak{m}_{\widetilde{\mathscr{C}}}\right)$ le faisceau associé. On désigne par $\operatorname{Mod}\left(\mathfrak{o}_{\mathscr{C}}\right)\left(\operatorname{resp} \operatorname{Mod}\left(\mathfrak{o}_{\widetilde{C}}\right)\right)$ la catégorie abélienne tensorielle des $\left(\mathfrak{o}_{\mathscr{\mathscr { C }}}\right)$-modules de $\widehat{\mathscr{C}}$ (resp. $\left(\mathfrak{o}_{\widetilde{\mathscr{C}}}\right)$-modules de $\widetilde{\mathscr{C}}$ ). Prenant pour $\mathfrak{o} \rightarrow \operatorname{End}\left(\mathfrak{o}_{\tilde{\mathscr{C}}}\right)\left(\right.$ resp. o $\rightarrow \operatorname{End}\left(\mathfrak{o}_{\tilde{\mathscr{C}}}\right)$ ) l'homomorphisme canonique, on appelle catégorie des $\alpha$-o $\mathfrak{o}_{\mathscr{C}}$-modules (resp. $\alpha-\mathfrak{o}_{\tilde{\mathscr{C}}}$-modules) et l'on $\operatorname{note} \alpha-\operatorname{Mod}\left(\mathfrak{o}_{\widehat{\mathscr{C}}}\right)\left(\operatorname{resp} . \alpha-\operatorname{Mod}\left(\mathfrak{o}_{\widetilde{\mathscr{C}}}\right)\right)$ le quotient $\operatorname{de} \operatorname{Mod}\left(\mathfrak{o}_{\mathscr{\mathscr { C }}}\right)\left(\operatorname{resp} \operatorname{Mod}\left(\mathfrak{o}_{\widetilde{\mathscr{C}}}\right)\right)$ par la sous-catégorie épaisse des modules $\alpha$-nuls.

12.7. On appelle catégorie des préfaisceaux de $\alpha$-o-modules sur $\mathscr{C}$ et l'on note $\alpha-\widehat{\mathscr{C}}$ la catégorie des préfaisceaux sur $\mathscr{C}$ à valeurs dans la catégorie $\alpha-\operatorname{Mod}(\mathfrak{o})$,

i.e. la catégorie des foncteurs de $\mathscr{C}^{\circ}$ à valeurs dans $\alpha-\operatorname{Mod}(\mathfrak{o})[3,1.4 .16]$.

On dit qu'un préfaisceau de $\alpha$-o-modules $F$ sur $\mathscr{C}$ est séparé (resp. un faisceau) si pour tout objet $X$ de $\mathscr{C}$ et tout crible couvrant $\mathscr{R}$ de $X$, le morphisme canonique

$$
F(X) \rightarrow \lim _{(Y, u) \in(\mathscr{C} / \mathscr{R})^{\circ}} F(Y)
$$

est un monomorphisme (resp. isomorphisme) (cf. [3, 1.4.21 et 1.4.24]). On note $\alpha-\widetilde{\mathscr{C}}$ la sous-catégorie pleine de $\alpha-\widehat{\mathscr{C}}$ des faisceaux de $\alpha$-o-modules sur $\mathscr{C}$. 
Le foncteur $\alpha: \operatorname{Mod}(\mathfrak{o}) \rightarrow \alpha-\operatorname{Mod}(\mathfrak{o})$ (12.1.1) définit un foncteur exact et monoïdal

$$
\widehat{\alpha}: \operatorname{Mod}\left(\mathfrak{o}_{\widehat{C}}\right) \rightarrow \alpha-\widehat{\mathscr{C}} .
$$

D'après [3, 1.4.9(i)], celui-ci transforme les faisceaux de o-modules en des faisceaux de $\alpha$-o-modules. Il induit donc un foncteur

$$
\tilde{\alpha}: \operatorname{Mod}\left(\mathfrak{o}_{\tilde{\mathscr{C}}}\right) \rightarrow \alpha-\tilde{\mathscr{C}} \text {. }
$$

Ce dernier transforme les $\alpha$-isomorphismes en des isomorphismes [3, 1.4.23(iii)]. Il induit une équivalence de catégories [3, 1.4.35]

$$
\alpha-\operatorname{Mod}\left(\mathfrak{o}_{\tilde{\mathscr{C}}}\right) \stackrel{\sim}{\rightarrow} \alpha-\tilde{\mathscr{C}}
$$

On munit $\alpha-\tilde{\mathscr{C}}$ de la structure de catégorie abélienne tensorielle déduite de celle $\operatorname{de} \alpha-\operatorname{Mod}\left(\mathfrak{o}_{\tilde{\mathscr{C}}}\right)(2.20)$ via l'équivalence (12.7.3).

En vertu de $[3,1.4 .31]$, le foncteur canonique d'inclusion $\iota: \alpha-\tilde{\mathscr{C}} \rightarrow \alpha-\widehat{\mathscr{C}}$ admet un adjoint à gauche

$$
\overline{\mathrm{a}}: \alpha-\widehat{\mathscr{C}} \rightarrow \alpha-\tilde{\mathscr{C}},
$$

dit foncteur 'faisceau de $\alpha$-o-modules associé'. On désigne par $i: \operatorname{Mod}\left(\mathfrak{o}_{\widetilde{C}}\right)$ $\rightarrow \operatorname{Mod}\left(\mathfrak{o}_{\widehat{\mathscr{C}}}\right)$ le foncteur canonique et par $\mathrm{a}: \operatorname{Mod}\left(\mathfrak{o}_{\widehat{\mathscr{C}}}\right) \rightarrow \operatorname{Mod}\left(\mathfrak{o}_{\tilde{\mathscr{C}}}\right)$ le foncteur 'faisceau de o-modules associé'. On a alors des diagrammes commutatifs à isomorphismes près $[3,1.4 .35$ et 1.4 .36$]$
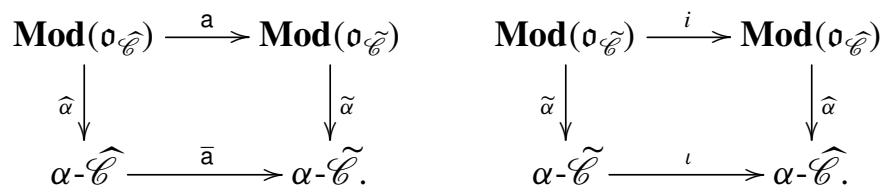

12.8. Les foncteurs $\sigma_{*}$ (12.1.3) et $\sigma_{!}(12.1 .4)$ (pour les o-modules) induisent des foncteurs que l'on note respectivement

$$
\begin{gathered}
\widehat{\sigma}_{*}: \alpha-\widehat{\mathscr{C}} \rightarrow \operatorname{Mod}\left(\mathfrak{o}_{\widehat{\mathscr{C}}}\right), \\
\widehat{\sigma}_{!}: \alpha-\widehat{\mathscr{C}} \rightarrow \operatorname{Mod}\left(\mathfrak{o}_{\widehat{\mathscr{C}}}\right) .
\end{gathered}
$$

En vertu de [3, 1.4.9(i)], $\widehat{\sigma}_{*}$ transforme les faisceaux de $\alpha$-o-modules en des faisceaux de o-modules. Il définit donc un foncteur

$$
\widetilde{\sigma}_{*}: \alpha-\tilde{\mathscr{C}} \rightarrow \operatorname{Mod}\left(\mathfrak{o}_{\tilde{\mathscr{C}}}\right)
$$

On désigne par $\widetilde{\sigma}_{!}$le foncteur

$$
\widetilde{\sigma}_{!}: \alpha-\tilde{\mathscr{C}} \rightarrow \operatorname{Mod}\left(\mathfrak{o}_{\tilde{\mathscr{C}}}\right) \quad M \mapsto \mathfrak{m}_{\tilde{\mathscr{C}}} \otimes_{\mathfrak{o}_{\widetilde{\mathscr{C}}}} \widetilde{\sigma}_{*}(M)
$$


On notera que $\widetilde{\sigma}_{!}$s'identifie au composé du foncteur $\widehat{\sigma}_{!}$(12.8.2) et du foncteur 'faisceau associé' a. D'après 12.2, le foncteur $\widehat{\sigma}_{*}$ (resp. $\widehat{\sigma}_{!}$) est un adjoint à droite (resp. à gauche) de $\widehat{\alpha}$ (cf. [3, 1.4.19]); les morphismes d'adjonction $\widehat{\alpha} \circ \widehat{\sigma}_{*} \rightarrow$ id et id $\rightarrow \widehat{\alpha} \circ \widehat{\sigma}_{!}$sont des isomorphismes. Le foncteur $\widetilde{\sigma}_{*}$ est un adjoint à droite de $\widetilde{\alpha}$ et le morphisme d'adjonction $\widetilde{\alpha} \circ \widetilde{\sigma}_{*} \rightarrow$ id est un isomorphisme [3, 1.4.35].

LEMME 12.9. (i) Le foncteur $\widetilde{\sigma}_{!}$(12.8.4) est un adjoint à gauche de $\widetilde{\alpha}$ (12.7.2).

(ii) Le morphisme d'adjonction id $\rightarrow \widetilde{\alpha} \circ \widetilde{\sigma}_{!}$est un isomorphisme.

Preuve. Soit $M$ un faisceau de $\alpha$-o-modules sur $\mathscr{C}$ et $N$ un $\mathfrak{o}_{\widetilde{C}}$-module. On a $\widetilde{\sigma}_{!}(M)=\mathrm{a}\left(\widehat{\sigma}_{!}(\iota(M))\right)$.

(i) Par adjonction et (12.7.5), on a des isomorphismes

$$
\begin{aligned}
\operatorname{Hom}_{\mathfrak{o}_{\widetilde{\mathscr{C}}}}\left(\mathrm{a}\left(\widehat{\sigma}_{!}(l(M))\right), N\right) & \simeq \operatorname{Hom}_{\mathfrak{o}_{\widetilde{\mathscr{C}}}}\left(\widehat{\sigma}_{!}(l(M)), i(N)\right) \\
& \simeq \operatorname{Hom}_{\alpha-\widehat{\mathscr{C}}}(l(M), \widehat{\alpha}(i(N))) \\
& \simeq \operatorname{Hom}_{\alpha-\widetilde{\mathscr{C}}}(M, \widetilde{\alpha}(N)) .
\end{aligned}
$$

(ii) Le morphisme d'adjonction $\iota(M) \rightarrow \widehat{\alpha}\left(\widehat{\sigma}_{!}(\iota(M))\right)$ est un isomorphisme. Compte tenu de (12.7.5), on a des isomorphismes

$$
M \stackrel{\sim}{\rightarrow} \overline{\mathrm{a}}\left(\widehat{\alpha}\left(\widehat{\sigma}_{!}(\iota(M))\right)\right) \simeq \widetilde{\alpha}\left(\widetilde{\sigma}_{!}(M)\right) .
$$

12.10. La donnée d'un monoïde commutatif unitaire de $\alpha$ - $\widetilde{\mathscr{C}}$ est équivalente à la donnée d'un préfaisceau sur $\mathscr{C}$ à valeurs dans $\alpha$-Alg(o) (12.4.1) dont le préfaisceau de $\alpha$-o-module sous-jacent est un faisceau (cf. [3, 1.4.40]). On note $\operatorname{Alg}(\alpha-\widetilde{\mathscr{C}})$ la catégorie des monoïdes commutatifs unitaires de $\alpha-\dot{\mathscr{C}}$.

De même, la donnée d'un monoïde commutatif unitaire de $\operatorname{Mod}\left(\mathfrak{o}_{\mathscr{C}}\right)$ est équivalente à la donnée d'une $\left(\mathfrak{o}_{\tilde{\mathscr{C}}}\right)$-algèbre de $\widetilde{\mathscr{C}}$. On note $\operatorname{Alg}\left(\mathfrak{o}_{\tilde{\mathscr{C}}}\right)$ la catégorie des monoïdes commutatifs unitaires de $\operatorname{Mod}\left(\mathfrak{o}_{\mathscr{C}}\right)$.

Le foncteur $\widetilde{\alpha}$ (12.7.2) étant monoïdal, il induit un foncteur que l'on note encore

$$
\widetilde{\alpha}: \operatorname{Alg}\left(\mathfrak{o}_{\tilde{C}}\right) \rightarrow \operatorname{Alg}(\alpha-\tilde{\mathscr{C}}) .
$$

Compte tenu de 12.4 , le foncteur $\widetilde{\sigma}_{*}$ (12.8.3) induit un foncteur que l'on note encore

$$
\widetilde{\sigma}_{*}: \operatorname{Alg}(\alpha-\tilde{\mathscr{C}}) \rightarrow \operatorname{Alg}\left(\mathfrak{o}_{\tilde{\mathscr{C}}}\right)
$$

D'après [3, 1.4.40], $\widetilde{\sigma}_{*}$ est un adjoint à droite de $\widetilde{\alpha}$; le morphisme d'adjonction $\widetilde{\alpha} \circ \widetilde{\sigma}_{*} \rightarrow$ id est un isomorphisme et le morphisme d'adjonction id $\rightarrow \widetilde{\sigma}_{*} \circ \widetilde{\alpha}$ induit un isomorphisme $\widetilde{\sigma} \stackrel{\sim}{\rightarrow} \widetilde{\alpha} \circ \widetilde{\sigma}_{*} \circ \widetilde{\alpha}$. 
12.11. Dans la suite de cette section, on fixe une $\mathfrak{o}_{\widetilde{\mathscr{C}}}$-algèbre $A$ de $\widetilde{\mathscr{C}}$. On désigne $\operatorname{par} \operatorname{Mod}(A)$ la catégorie abélienne tensorielle des $A$-modules de $\widetilde{\mathscr{C}}$. Prenant pour $\varphi: \mathfrak{o} \rightarrow \Gamma(\widetilde{\mathscr{C}}, A)$ l'homomorphisme canonique, on appelle catégorie des $\alpha$ - $A$ modules et l'on note $\alpha-\operatorname{Mod}(A)$ le quotient de la catégorie abélienne $\operatorname{Mod}(A)$ par la sous-catégorie épaisse des $A$-modules $\alpha$-nuls.

On pose $A^{\alpha}=\widetilde{\alpha}(A)(12.10 .1)$ et on désigne par $\operatorname{Mod}\left(A^{\alpha}\right)$ la catégorie des $A^{\alpha}$ modules unitaires de $\alpha-\widetilde{\mathscr{C}}$. La donnée d'une structure de $A^{\alpha}$-module unitaire sur un faisceau de $\alpha$-o-modules $M \operatorname{sur} \mathscr{C}$ est équivalente à la donnée pour tout $X \in$ $\mathbf{O b}(\mathscr{C})$ d'une structure de $\alpha(A(X))$-module unitaire sur $M(X)$ dans le sens de 12.5 telle que pour tout morphisme $X \rightarrow Y$ de $\mathscr{C}$, le morphisme $M(Y) \rightarrow M(X)$ soit linéaire relativement au morphisme de $\mathfrak{o}^{\alpha}$-algèbres $\alpha(A(Y)) \rightarrow \alpha(A(X))$, où $\alpha: \operatorname{Alg}(\mathfrak{o}) \rightarrow \alpha-\operatorname{Alg}(\mathfrak{o})$ est le foncteur (12.4.1) (cf. [3, 1.4.41]).

Le foncteur $\widetilde{\alpha}$ (12.7.2) étant monoïdal, il définit un foncteur

$$
\widetilde{\alpha}_{A}: \operatorname{Mod}(A) \rightarrow \operatorname{Mod}\left(A^{\alpha}\right) .
$$

Celui-ci transforme les $\alpha$-isomorphismes en des isomorphismes (cf. [3, 1.4.23(iii)]). Il induit une équivalence de catégories [3, 1.4.41]

$$
\alpha-\operatorname{Mod}(A) \stackrel{\sim}{\rightarrow} \operatorname{Mod}\left(A^{\alpha}\right) .
$$

Dans la suite de cette section, pour tout $A$-module $M$, on pose abusivement $M^{\alpha}=$ $\tilde{\alpha}_{A}(M)$.

12.12. On pose $A^{\prime}=\widetilde{\sigma}_{*}\left(A^{\alpha}\right)$ (12.10.2). D'après 12.10 , on a un homomorphisme canonique de $\mathfrak{o}_{\tilde{\mathscr{C}}}$-algèbres $\lambda: A \rightarrow A^{\prime}$, qui induit un isomorphisme $\widetilde{\alpha}(A) \stackrel{\sim}{\rightarrow}$ $\widetilde{\alpha}\left(A^{\prime}\right)$. Compte tenu de 12.5 , le foncteur $\widetilde{\sigma}_{*}$ (12.8.3) induit un foncteur (cf. [3, 1.4.41])

$$
\widetilde{\tau}_{*}^{\prime}: \operatorname{Mod}\left(A^{\alpha}\right) \rightarrow \operatorname{Mod}\left(A^{\prime}\right)
$$

Composant avec le foncteur induit par $\lambda$, on obtient un foncteur

$$
\tilde{\tau}_{*}: \operatorname{Mod}\left(A^{\alpha}\right) \rightarrow \operatorname{Mod}(A)
$$

On désigne par $\widetilde{\tau}_{!}$le foncteur

$$
\tilde{\tau}_{!}: \operatorname{Mod}\left(A^{\alpha}\right) \rightarrow \operatorname{Mod}(A), \quad M \mapsto \mathfrak{m}_{\tilde{\mathscr{C}}} \otimes_{\mathfrak{o}_{\tilde{\mathscr{C}}}} \widetilde{\tau}_{*}(M) .
$$

Pour tous $A^{\alpha}$-modules $M$ et $N$, on pose

$$
\operatorname{Hom}_{A^{\alpha}}(M, N)=\operatorname{Hom}_{M o d}\left(A^{\alpha}\right)(M, N),
$$

qui est naturellement muni d'une structure de $\Gamma(\widetilde{\mathscr{C}}, A)$-module, en particulier d'une structure de o-module. 
12.13. Soit $A \rightarrow B$ un morphisme de $\mathfrak{o}_{\widetilde{\mathscr{C}}}$-algèbres. On note $B^{\alpha}=\widetilde{\alpha}(\underset{\widetilde{C}}{B})(12.10 .1)$ et on désigne par $\operatorname{Mod}\left(B^{\alpha}\right)$ la catégorie des $B^{\alpha}$-modules de $\alpha-\widetilde{\mathscr{C}}$. On a des diagrammes commutatifs
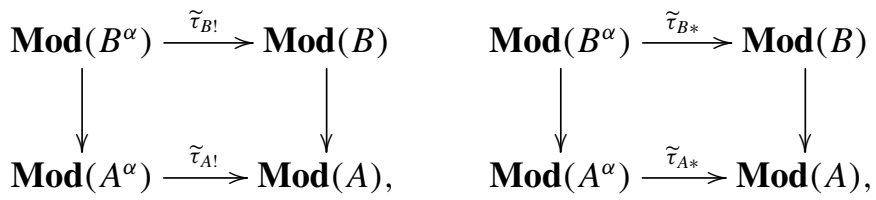

où les flèches verticales sont des foncteurs d'oubli, et $\widetilde{\tau}_{B *}, \widetilde{\tau}_{B !}$, $\widetilde{\tau}_{A *}$ et $\widetilde{\tau}_{A !}$ désignent les foncteurs (12.12.1) et (12.12.2) relatifs à $B$ et $A$ respectivement. On peut donc omettre les indices $B$ et $A$ dans les notations de $\widetilde{\tau}_{B *}, \widetilde{\tau}_{B !}, \widetilde{\tau}_{A *}$ et $\widetilde{\tau}_{A !}$.

Lemme $12.14[3,1.4 .23]$. (i) Pour qu'un morphisme de A-modules $f: M \rightarrow$ $N$ soit un $\alpha$-isomorphisme, il faut et il suffit que le morphisme $\mathfrak{m}_{\widetilde{\mathscr{C}}} \otimes_{\mathfrak{o}_{\widetilde{G}}} M \rightarrow$ $\mathfrak{m}_{\tilde{\mathscr{C}}} \otimes_{\mathfrak{o}_{\widetilde{\mathscr{G}}}} N$ induit par $f$ soit un isomorphisme.

(ii) Pour tous A-modules $M$ et $N$, on a un isomorphisme o-linéaire canonique

$$
\operatorname{Hom}_{A^{\alpha}}\left(M^{\alpha}, N^{\alpha}\right) \stackrel{\sim}{\rightarrow} \operatorname{Hom}_{A}\left(\mathfrak{m}_{\tilde{\mathscr{C}}} \otimes_{\mathfrak{o}_{\widetilde{\mathscr{C}}}} M, N\right)
$$

PROPOSITION 12.15. (i) Le foncteur $\tilde{\tau}_{*}(12.12 .1)$ est un adjoint à droite du foncteur $\widetilde{\alpha}_{A}(12.11 .1)$.

(ii) Le morphisme d'adjonction $\widetilde{\alpha}_{A} \circ \widetilde{\tau}_{*} \rightarrow$ id est un isomorphisme.

(iii) Le foncteur $\widetilde{\tau}_{!}(12.12 .2)$ est un adjoint à gauche du foncteur $\widetilde{\alpha}_{A}$.

(iv) Le morphisme d'adjonction id $\rightarrow \widetilde{\alpha}_{A} \circ \tilde{\tau}_{!}$est un isomorphisme.

Preuve. Les assertions (i) et (ii) sont démontrées dans [3, 1.4.41].

(iii) Soit $M$ un $A$-module et $N$ un $A^{\alpha}$-module. D'après (ii) et (12.14.1), on a des isomorphismes

$$
\begin{aligned}
\operatorname{Hom}_{A^{\alpha}}\left(N, M^{\alpha}\right) & \stackrel{\sim}{\rightarrow} \operatorname{Hom}_{A^{\alpha}}\left(\left(\widetilde{\tau}_{*}(N)\right)^{\alpha}, M^{\alpha}\right) \\
& \stackrel{\sim}{\rightarrow} \operatorname{Hom}_{A}\left(\mathfrak{m}_{\tilde{\mathscr{C}}} \otimes_{\mathfrak{o}_{\widetilde{\mathscr{C}}}} \widetilde{\tau}_{*}(N), M\right) \\
& \stackrel{\sim}{\rightarrow} \operatorname{Hom}_{A}\left(\widetilde{\tau}_{!}(N), M\right) .
\end{aligned}
$$

(iv) On notera que, pour tout $A^{\alpha}$-module $M$, le $\alpha$-o-module sous-jacent à $\left(\widetilde{\tau}_{!}(M)\right)^{\alpha}$ s'identifie canoniquement à $\widetilde{\alpha}\left(\widetilde{\sigma}_{!}(M)\right)$. L'assertion résulte du fait que le morphisme d'adjonction id $\rightarrow \widetilde{\alpha} \circ \widetilde{\sigma}_{!}$est un isomorphisme (cf. 12.9(ii)). 
Corollaire 12.16. (i) Les foncteurs $\widetilde{\alpha}_{A}$, $\widetilde{\tau}_{!}$sont exacts et le foncteur $\widetilde{\tau}_{*}$ est exact à gauche.

(ii) Les foncteurs $\widetilde{\alpha}_{A}$ et $\widetilde{\tau}_{*}$ transforment les objets injectifs en des objets injectifs. En particulier, $\operatorname{Mod}\left(A^{\alpha}\right)$ a suffisamment d'injectifs.

(iii) Le morphisme d'adjonction id $\rightarrow \widetilde{\tau}_{*} \circ \widetilde{\alpha}_{A}$ (resp. $\widetilde{\tau}_{!} \circ \widetilde{\alpha}_{A} \rightarrow$ id) induit un isomorphisme $\widetilde{\alpha} \stackrel{\sim}{\rightarrow} \widetilde{\alpha}_{A} \circ \widetilde{\tau}_{*} \circ \widetilde{\alpha}_{A}$ (resp. $\widetilde{\alpha}_{A} \circ \widetilde{\tau}_{!} \circ \widetilde{\alpha}_{A} \stackrel{\sim}{\rightarrow} \widetilde{\alpha}_{A}$ ).

Preuve. (i) Compte tenu de $12.15, \widetilde{\alpha}_{A}$ est exact et $\widetilde{\tau}_{*}$ (resp. $\widetilde{\tau}_{!}$) est exact à gauche (resp. à droite). Le $\mathfrak{o}_{\tilde{\mathscr{C}}}$-module $\mathfrak{m}_{\tilde{\mathscr{C}}}$ étant plat [6, V 1.7.1], l'exactitude de $\widetilde{\tau}_{!}$ s'ensuit compte tenu de (12.12.2).

(ii) Le foncteur $\widetilde{\alpha}_{A}$ (resp. $\widetilde{\tau}_{*}$ ) est un adjoint à droite d'un foncteur exact, d'où l'assertion [6, V 0.2].

(iii) En effet, le morphisme composé

$$
\tilde{\alpha}_{A} \rightarrow \tilde{\alpha}_{A} \circ \tilde{\tau}_{*} \circ \tilde{\alpha}_{A} \rightarrow \tilde{\alpha}_{A}
$$

où les flèches sont déduites des morphismes d'adjonction, s'identifie au morphisme identique. L'assertion pour $\widetilde{\tau}_{*}$ s'ensuit compte tenu de 12.15(ii). La démonstration de l'assertion pour $\widetilde{\tau}_{!}$est similaire.

REMARQUE 12.17 . Le foncteur $\widetilde{\alpha}_{A}$ ne transforme pas les objets projectifs en des objets projectifs. En effet, considérons le topos ponctuel et prenons pour $A$ l'anneau $\mathfrak{o}$. En vertu de (12.1.5), pour tout $\mathfrak{o}$-module $M$, on a un isomorphisme canonique

$$
\operatorname{Hom}_{\mathfrak{o}^{\alpha}}\left(\mathfrak{o}^{\alpha}, M^{\alpha}\right) \stackrel{\sim}{\rightarrow} \operatorname{Hom}_{\mathfrak{o}}(\mathfrak{m}, M)
$$

Compte tenu de 3.3, $\mathfrak{o}^{\alpha}$ n'est pas projectif dans la catégorie $\alpha-\operatorname{Mod}(\mathfrak{o})$.

12.18. On désigne par $\mathbf{D}(\operatorname{Mod}(A))\left(\operatorname{resp} . \mathbf{D}\left(\operatorname{Mod}\left(A^{\alpha}\right)\right)\right)$ la catégorie dérivée de $\operatorname{Mod}(A)\left(\operatorname{resp} . \operatorname{Mod}\left(A^{\alpha}\right)\right)$. Les foncteurs exacts $\widetilde{\alpha}_{A}$ et $\widetilde{\tau}_{!}$induisent des foncteurs exacts entre catégories dérivées

$$
\begin{aligned}
& F: \mathbf{D}(\operatorname{Mod}(A)) \rightarrow \mathbf{D}\left(\operatorname{Mod}\left(A^{\alpha}\right)\right), \\
& G: \mathbf{D}\left(\operatorname{Mod}\left(A^{\alpha}\right)\right) \rightarrow \mathbf{D}(\operatorname{Mod}(A)) .
\end{aligned}
$$

Proposition 12.19. Le foncteur $G$ est un adjoint à gauche de $F$. 
Preuve. D'après $[19,1.1 .12]$, on a des identités triangulaires
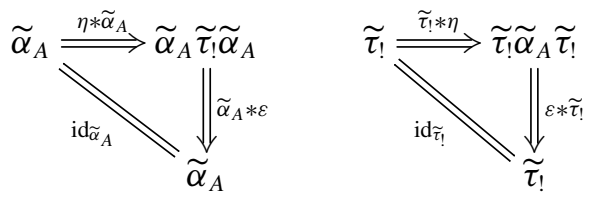

où $\eta$ et $\varepsilon$ désignent les transformations naturelles d'adjonction. Comme $\widetilde{\alpha}_{A}$ et $\widetilde{\tau}_{!}$ sont exacts, on en déduit des identités triangulaires similaires pour la paire $(G, F)$. La proposition s'ensuit compte tenu de [19, 1.1.14].

12.20. Rappelons que la catégorie abélienne $\operatorname{Mod}\left(A^{\alpha}\right)$ a suffisamment d'injectifs (12.16(ii)) et reprenons les définitions de 2.4 pour cette catégorie. Pour tous $A^{\alpha}$ modules $M, N$ et tout entier $i \geqslant 0$, on pose

$$
\operatorname{Ext}_{A^{\alpha}}^{i}(M, N)=\operatorname{Ext}_{\operatorname{Mod}\left(A^{\alpha}\right)}^{i}(M, N)
$$

qui est muni d'une structure de o-module.

D'après (2.3.1) et 12.19 , pour tout $A^{\alpha}$-module $M$, tout $A$-module $N$ et tout entier $i \geqslant 0$, on a un isomorphisme canonique

$$
\operatorname{Ext}_{A}^{i}\left(\widetilde{\tau}_{!}(M), N\right) \stackrel{\sim}{\rightarrow} \operatorname{Ext}_{A^{\alpha}}^{i}\left(M, N^{\alpha}\right) .
$$

Soit $M$ et $N$ deux $A$-modules. Le foncteur exact $\widetilde{\alpha}_{A}$ induit un morphisme canonique (2.3.2)

$$
\operatorname{Ext}_{A}^{i}(M, N) \rightarrow \operatorname{Ext}_{A^{\alpha}}^{i}\left(M^{\alpha}, N^{\alpha}\right) .
$$

Composant avec l'isomorphisme $\operatorname{Ext}_{A^{\alpha}}^{i}\left(M^{\alpha}, N^{\alpha}\right) \stackrel{\sim}{\rightarrow} \operatorname{Ext}_{A}^{i}\left(\widetilde{\tau}_{!}\left(M^{\alpha}\right), N\right)$, on obtient un morphisme canonique

$$
\operatorname{Ext}_{A}^{i}(M, N) \rightarrow \operatorname{Ext}_{A}^{i}\left(\widetilde{\tau}_{!}\left(M^{\alpha}\right), N\right) .
$$

On notera que celui-là est induit par le morphisme d'adjonction $G \circ F \rightarrow$ id. En particulier, il s'identifie au morphisme canonique induit par la flèche d'adjonction $\widetilde{\tau}_{!}\left(M^{\alpha}\right) \rightarrow M$.

Lemme 12.21. Soit $M$ et $N$ deux A-modules de $\tilde{\mathscr{C}}$. Le morphisme canonique (12.20.2) est un $\alpha$-isomorphisme de o-modules.

Preuve. Il suffit de démontrer que le morphisme (12.20.3) est un $\alpha$-isomorphisme. En vertu de 12.16(iii), le morphisme canonique $f: \widetilde{\tau}_{!}\left(M^{\alpha}\right) \rightarrow M$ est un $\alpha$ isomorphisme de $\operatorname{Mod}(A)$. D'après 2.19, pour tout $\gamma \in \mathfrak{m}$, il existe un morphisme 
$A$-linéaire $g_{\gamma}: M \rightarrow \widetilde{\tau}_{!}\left(M^{\alpha}\right)$ tel que $g_{\gamma} \circ f=\gamma^{2} \mathrm{id}_{M}$ et $f \circ g_{\gamma}=\gamma^{2} \mathrm{id}_{\widetilde{\tau}_{(}\left(M^{\alpha}\right)}$. On en déduit que le noyau et le conoyau de (12.20.3) sont annulés par $\gamma^{2}$, d'où le lemme.

COROLlaire 12.22. Le foncteur $\widetilde{\alpha}_{A}$ (12.11.1) induit une équivalence de catégories

$$
\operatorname{Mod}_{\mathbb{Q}}(A) \stackrel{\sim}{\rightarrow} \operatorname{Mod}_{\mathbb{Q}}\left(A^{\alpha}\right),
$$

où la source (resp. le but) désigne la catégorie des A-modules (resp. $A^{\alpha}$-modules) à isogénie près (2.16).

Preuve. Par définition, le foncteur est essentiellement surjectif. La pleine fidélité résulte de 12.21.

12.23. Soit $M$ un $A^{\alpha}$-module et $N$ un $A$-module. En vertu de 12.15(iv), on a des isomorphismes canoniques et fonctoriels de $A^{\alpha}$-modules

$$
\left(\widetilde{\tau}_{!}\left(M \otimes_{A^{\alpha}} N^{\alpha}\right)\right)^{\alpha} \stackrel{\sim}{\rightarrow} M \otimes_{A^{\alpha}} N^{\alpha}, \quad\left(\widetilde{\tau}_{!}(M)\right)^{\alpha} \otimes_{A^{\alpha}} N^{\alpha} \stackrel{\sim}{\rightarrow} M \otimes_{A^{\alpha}} N^{\alpha} .
$$

On en déduit, par 12.14(ii) et 12.15(iii), un isomorphisme canonique et fonctoriel de $A$-modules

$$
\mathfrak{m}_{\tilde{\mathscr{C}}} \otimes_{\mathfrak{o}_{\tilde{\mathscr{C}}}} \widetilde{\tau}_{!}\left(M \otimes_{A^{\alpha}} N^{\alpha}\right) \stackrel{\sim}{\rightarrow} \mathfrak{m}_{\tilde{\mathscr{C}}} \otimes_{\mathfrak{o}_{\tilde{\mathscr{C}}}} \widetilde{\tau}_{!}(M) \otimes_{A} N .
$$

Compte tenu de l'isomorphisme $\mathfrak{m} \otimes_{\mathfrak{o}} \mathfrak{m} \simeq \mathfrak{m}$ et de la définition de $\widetilde{\tau}_{!}$, celui-ci s'identifie à l'isomorphisme

$$
\tilde{\tau}_{!}\left(M \otimes_{A^{\alpha}} N^{\alpha}\right) \stackrel{\sim}{\rightarrow} \tilde{\tau}_{!}(M) \otimes_{A} N
$$

Proposition 12.24. Soit $n$ un entier $\geqslant 1$ et supposons que $p^{n} A=0$. Soit $M$ un A-module plat sur $\mathfrak{o}_{n}[6, \mathrm{~V} 1.7]$.

(i) Pour tout $\mathfrak{o}_{n}$-module $P$ et tout entier $i \geqslant 0$, on a un isomorphisme canonique

$$
\operatorname{Ext}_{\mathfrak{o}_{n}}^{i}\left(\mathfrak{m} / p^{n} \mathfrak{m}, P\right) \stackrel{\sim}{\rightarrow} \operatorname{Ext}_{\mathfrak{o}^{i}}^{i}(\mathfrak{m}, P)
$$

(ii) Le foncteur $\operatorname{Hom}_{A}(M,-): \operatorname{Mod}(A) \rightarrow \operatorname{Mod}\left(\mathfrak{o}_{n}\right)$ transforme les objets injectifs en objets injectifs.

(iii) Soit $N_{1}$ et $N_{2}$ deux A-modules. On a un isomorphisme canonique et fonctoriel

$$
\operatorname{Hom}_{A}\left(\mathfrak{m}_{\tilde{\mathscr{C}}} \otimes_{\mathfrak{o}_{\widetilde{\mathscr{C}}}} N_{1}, N_{2}\right) \stackrel{\sim}{\rightarrow} \operatorname{Hom}_{\mathfrak{o}}\left(\mathfrak{m}, \operatorname{Hom}_{A}\left(N_{1}, N_{2}\right)\right)
$$


(iv) Pour tout A-module $N$, on a une suite spectrale

$$
\mathrm{E}_{2}^{i, j}=\operatorname{Ext}_{\mathfrak{o}}^{i}\left(\mathfrak{m}, \operatorname{Ext}_{A}^{j}(M, N)\right) \Rightarrow \mathrm{E}^{i+j}=\operatorname{Ext}_{A}^{i+j}\left(\mathfrak{m}_{\widetilde{\mathscr{C}}} \otimes_{\mathfrak{o}_{\widetilde{\mathscr{C}}}} M, N\right)
$$

Preuve. (i) Le foncteur $\operatorname{Hom}_{\mathfrak{o}}(-, P): \operatorname{Mod}(\mathfrak{o}) \rightarrow \operatorname{Mod}(\mathfrak{o})$ s'identifie au foncteur composé

$$
\operatorname{Mod}(\mathfrak{o}) \stackrel{-\otimes_{\mathfrak{o}} \mathfrak{o}_{n}}{\longrightarrow} \operatorname{Mod}\left(\mathfrak{o}_{n}\right) \stackrel{\operatorname{Hom}_{\mathfrak{o}_{n}}(-, P)}{\longrightarrow} \operatorname{Mod}(\mathfrak{o}) .
$$

Comme $-\otimes_{\mathfrak{o}} \mathfrak{o}_{n}$ envoie les objets projectifs sur des objets projectifs, on en déduit une suite spectrale

$$
\operatorname{Ext}_{\mathfrak{o}_{n}}^{i}\left(\operatorname{Tor}_{j}^{\mathfrak{o}}\left(\mathfrak{m}, \mathfrak{o}_{n}\right), P\right) \Rightarrow \operatorname{Ext}_{\mathfrak{o}}^{i+j}(\mathfrak{m}, P) .
$$

L'assertion résulte alors de la platitude de $\mathfrak{m}$.

(ii) Posons $\mathfrak{o}_{\widetilde{\mathscr{C}}, n}=\mathfrak{o}_{\tilde{\mathscr{C}}} / p^{n} \mathfrak{o}_{\tilde{\mathscr{C}}}$. Soit $P$ un $\mathfrak{o}_{n}$-module et $P_{\widetilde{\mathscr{C}}}$ le faisceau associé au préfaisceau constant de valeur $P$ sur $\mathscr{C}$. Pour tout $A$-module $N$, par adjonction [6, IV 13.4.1], on a un isomorphisme canonique

$$
\operatorname{Hom}_{\mathfrak{o}_{\widetilde{\mathscr{C}}, n}}\left(P_{\widetilde{\mathscr{C}}}, N\right) \stackrel{\sim}{\rightarrow} \operatorname{Hom}_{\mathfrak{o}_{n}}(P, \Gamma(\widetilde{\mathscr{C}}, N))
$$

Soit $I$ un $A$-module injectif de $\tilde{\mathscr{C}}$. Pour tout $\mathfrak{o}_{n}$-module $P$, on en déduit un isomorphisme canonique

$$
\operatorname{Hom}_{\mathfrak{o}_{\widetilde{\mathscr{C}}, n}}\left(P_{\widetilde{\mathscr{C}}}, \mathscr{H} \operatorname{om}_{A}(M, I)\right) \stackrel{\sim}{\rightarrow} \operatorname{Hom}_{\mathfrak{o}_{n}}\left(P, \operatorname{Hom}_{A}(M, I)\right)
$$

Par ailleurs, on a un isomorphisme canonique [6, IV 12.14]

$$
\operatorname{Hom}_{A}\left(P_{\widetilde{\mathscr{C}}} \otimes_{\mathfrak{o}_{\widetilde{\mathscr{C}}, n}} M, I\right) \stackrel{\sim}{\rightarrow} \operatorname{Hom}_{\mathfrak{o}_{\widetilde{\mathscr{C}}, n}}\left(P_{\widetilde{\mathscr{C}}}, \mathscr{H}_{0} \mathrm{om}_{A}(M, I)\right)
$$

L'injectivité de $\operatorname{Hom}_{A}(M, I)$ résulte de celle de $I$ et de la platitude de $M$.

(iii) En vertu de [6, IV 12.14], on a un isomorphisme canonique et fonctoriel

$$
\operatorname{Hom}_{A}\left(\mathfrak{m}_{\tilde{\mathscr{C}}} \otimes_{\mathfrak{o}_{\widetilde{\mathscr{C}}}} N_{1}, N_{2}\right) \stackrel{\sim}{\rightarrow} \operatorname{Hom}_{\mathfrak{o}_{\widetilde{\mathscr{C}}}}\left(\mathfrak{m}_{\tilde{\mathscr{C}}}, \mathscr{H}_{0} \mathrm{~m}_{A}\left(N_{1}, N_{2}\right)\right) .
$$

Par adjonction [6, IV 13.4.1], on a un isomorphisme canonique et fonctoriel

$$
\operatorname{Hom}_{\mathfrak{o}_{\tilde{\mathscr{C}}}}\left(\mathfrak{m}_{\tilde{\mathscr{C}}}, \mathscr{H}_{\mathrm{om}}\left(N_{1}, N_{2}\right)\right) \stackrel{\sim}{\rightarrow} \operatorname{Hom}_{\mathfrak{o}}\left(\mathfrak{m}, \operatorname{Hom}_{A}\left(N_{1}, N_{2}\right)\right) .
$$

(iv) D'après (iii), le foncteur

$$
\operatorname{Hom}_{A}\left(\mathfrak{m}_{\tilde{\mathscr{C}}} \otimes_{\mathfrak{o}_{\widetilde{\mathscr{C}}}} M,-\right): \operatorname{Mod}(A) \rightarrow \operatorname{Mod}\left(\mathfrak{o}_{n}\right)
$$

s'identifie au foncteur composé

$$
\operatorname{Mod}(A) \stackrel{\operatorname{Hom}_{A}(M,-)}{\longrightarrow} \operatorname{Mod}\left(\mathfrak{o}_{n}\right) \stackrel{\operatorname{Hom}_{\mathfrak{o}_{n}}\left(\mathfrak{m} / p^{n} \mathfrak{m},-\right)}{\longrightarrow} \operatorname{Mod}\left(\mathfrak{o}_{n}\right)
$$


Comme $\operatorname{Hom}_{A}(M,-)$ envoie les objets injectifs sur des objets injectifs, on en déduit une suite spectrale

$$
\operatorname{Ext}_{\mathfrak{o}_{n}}^{i}\left(\mathfrak{m} / p^{n} \mathfrak{m}, \operatorname{Ext}_{A}^{j}(M, N)\right) \Rightarrow \operatorname{Ext}_{A}^{i+j}\left(\mathfrak{m}_{\tilde{\mathscr{C}}} \otimes_{\mathfrak{o}_{\widetilde{\mathscr{C}}}} M, N\right)
$$

La suite spectrale (12.24.2) s'ensuit compte tenu de (i).

12.25. Soit $\varphi:\left(\widetilde{\mathscr{C}^{\prime}}, A^{\prime}\right) \rightarrow(\widetilde{\mathscr{C}}, A)$ un morphisme de topos annelés. On note $\alpha$ - $\widetilde{\mathscr{C}}^{\prime}$ la catégorie des faisceaux de $\alpha$-o-modules de $\widetilde{\mathscr{C}^{\prime}}(12.7)$ et on pose $A^{\prime \alpha}=$ $\widetilde{\alpha}\left(A^{\prime}\right)$ (12.10.1). On désigne par $\operatorname{Mod}\left(A^{\prime \alpha}\right)$ la catégorie des $A^{\prime \alpha}$-modules de $\alpha-\widetilde{\mathscr{C}^{\prime}}$. En vertu de 12.14(i), le foncteur $\varphi^{*}: \operatorname{Mod}(A) \rightarrow \operatorname{Mod}\left(A^{\prime}\right)$ envoie les $\alpha$-isomorphismes sur des $\alpha$-isomorphismes. Il induit donc un foncteur

$$
\psi: \operatorname{Mod}\left(A^{\alpha}\right) \rightarrow \operatorname{Mod}\left(A^{\prime \alpha}\right)
$$

qui s'insère dans un diagramme commutatif

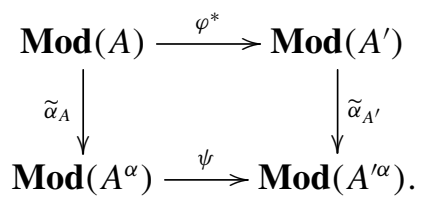

Compte tenu de 12.15(iv), pour tout $A^{\alpha}$-module $M$, on a un isomorphisme canonique et fonctoriel

$$
\psi(M) \stackrel{\sim}{\rightarrow}\left(\varphi^{*}\left(\tilde{\tau}_{A !}(M)\right)\right)^{\alpha} .
$$

Le morphisme adjoint de cet isomorphisme

$$
\tilde{\tau}_{A^{\prime} !}(\psi(M)) \rightarrow \varphi^{*}\left(\widetilde{\tau}_{A !}(M)\right)
$$

est un $\alpha$-isomorphisme en vertu de 12.15(iii). C'est même un isomorphisme en vertu de 12.14(ii) et de l'isomorphisme $\mathfrak{m} \otimes_{\mathfrak{o}} \mathfrak{m} \simeq \mathfrak{m}$.

12.26. Conservons les notations de 12.25 et supposons que le morphisme $\varphi$ soit plat, i.e. le foncteur $\varphi^{*}: \operatorname{Mod}(A) \rightarrow \operatorname{Mod}\left(A^{\prime}\right)$ soit exact [6, V 1.8]. D'après [20, III.1, Cor. 3], le foncteur $\psi$ (12.25.1) est exact. En vertu de (12.25.2) et (12.25.4), pour tout $A^{\alpha}$-module $M$, tout $A$-module $N$ et tout entier $i \geqslant 0$, les isomorphismes (12.20.1) induisent un diagramme commutatif

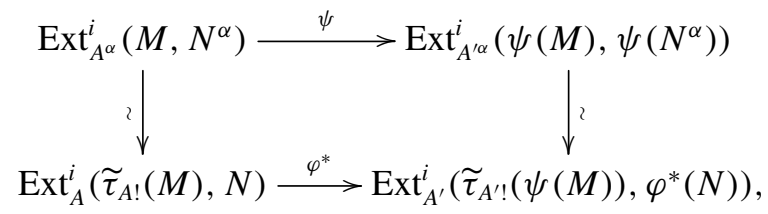


où les flèches horizontales sont les morphismes canoniques induits par les foncteurs exacts $\varphi^{*}$ et $\psi(2.3 .2)$.

12.27. Conservons les notations et les hypothèses de 12.26 et supposons de plus que $p^{n} A=0$. Soit $M$ un $A$-module qui est plat sur $\mathfrak{o}_{n}$ et $N$ un $A$-module. Comme $\varphi^{*}$ est exact, le $A^{\prime}$-module $\varphi^{*}(M)$ est encore plat sur $\mathfrak{o}_{n}$. On désigne par E (resp. $\mathrm{E}^{\prime}$ ) la suite spectrale (12.24.2) associée aux $A$-modules $M$ et $N$ (resp. $A^{\prime}$-modules $\varphi^{*}(M)$ et $\left.\varphi^{*}(N)\right)$. Le foncteur exact $\varphi^{*}$ induit un morphisme de suites spectrales (cf. 15.5)

$$
u=\left(u_{r}^{i, j}, u^{n}\right): \mathrm{E} \rightarrow \mathrm{E}^{\prime}
$$

dont les morphismes

$$
\begin{aligned}
u_{2}^{i, j}: \operatorname{Ext}_{\mathfrak{o}}^{i}\left(\mathfrak{m}, \operatorname{Ext}_{A}^{j}(M, N)\right) \rightarrow \operatorname{Ext}_{\mathfrak{o}}^{i}\left(\mathfrak{m}, \operatorname{Ext}_{A^{\prime}}^{j}\left(\varphi^{*}(M), \varphi^{*}(N)\right)\right) \\
u^{n}: \operatorname{Ext}_{A}^{n}\left(\mathfrak{m}_{\widetilde{\mathscr{C}}} \otimes_{\mathfrak{o}_{\widetilde{\mathscr{C}}}} M, N\right) \rightarrow \operatorname{Ext}_{\mathcal{A}^{\prime}}^{n}\left(\mathfrak{m}_{\widetilde{\mathscr{C}}^{\prime}} \otimes_{\mathfrak{o}_{\widetilde{\mathscr{G}}}} \varphi^{*}(M), \varphi^{*}(N)\right)
\end{aligned}
$$

s'identifient aux morphismes canoniques induits par $\varphi^{*}$ (2.3.2). Compte tenu de l'isomorphisme canonique $\mathfrak{m}_{\tilde{\mathscr{C}}} \otimes_{\mathfrak{o}_{\widetilde{\mathscr{C}}}} M \simeq \widetilde{\tau}_{!}\left(M^{\alpha}\right)$ et (12.26.1), le morphisme $u^{n}$ s'identifie au morphisme canonique induit par $\psi$ :

$$
\operatorname{Ext}_{A^{\alpha}}^{n}\left(M^{\alpha}, N^{\alpha}\right) \rightarrow \operatorname{Ext}_{A^{\prime \alpha}}^{n}\left(\psi\left(M^{\alpha}\right), \psi\left(N^{\alpha}\right)\right) .
$$

DÉfinition $12.28[3,1.5 .3]$. Soit $M$ un $A$-module de $\widetilde{\mathscr{C}}$ et $\gamma \in \mathfrak{m}$.

(i) On dit que $M$ est de type $\gamma$-fini s'il existe un raffinement $\left(U_{i}\right)_{i \in I}$ de l'objet final de $\widetilde{\mathscr{C}}$ tel que, pour tout $i \in I$, il existe un $\left(A \mid U_{i}\right)$-module libre de type fini $N_{i}$ et un morphisme $\left(A \mid U_{i}\right)$-linéaire

$$
N_{i} \rightarrow M \mid U_{i}
$$

dont le conoyau est annulé par $\gamma$.

(ii) On dit que $M$ est de type $\alpha$-fini s'il est de type $\gamma$-fini pour tout $\gamma \in \mathfrak{m}$.

DÉFInItion 12.29. (i) On dit qu'un $A$-module $M$ est plat (resp. $\alpha$-plat) si, pour tout morphisme injectif de $A$-modules $f: N_{1} \rightarrow N_{2}$, le noyau de $\operatorname{id}_{M} \otimes f: M \otimes_{A} N_{1} \rightarrow M \otimes_{A} N_{2}$ est nul (resp. $\alpha$-nul).

(ii) On dit qu'un $A^{\alpha}$-module $M$ est plat si, pour tout morphisme injectif de $A^{\alpha}$ modules $f: N_{1} \rightarrow N_{2}$, le noyau de $\operatorname{id}_{M} \otimes f: M \otimes_{A^{\alpha}} N_{1} \rightarrow M \otimes_{A^{\alpha}} N_{2}$ est nul.

Lemme 12.30. (i) Pour qu'un A-module M soit $\alpha$-plat, il faut et il suffit que $M^{\alpha}$ soit un $A^{\alpha}$-module plat. 
(ii) Pour qu'un $A^{\alpha}$-module $N$ soit plat, il faut et il suffit que $\widetilde{\tau}_{!}(N)$ soit un $A$ module plat.

Preuve. (i) Supposons que $M$ soit un $A$-module $\alpha$-plat et soit $f: N_{1} \rightarrow N_{2}$ une injection de $A^{\alpha}$-modules. D'après 12.16(i), $f$ induit une injection de $A$-modules $\tau_{!}\left(N_{1}\right) \rightarrow \tau_{!}\left(N_{2}\right)$. On en déduit que le noyau de $\mathrm{id}_{M} \otimes \tau_{!}(f): M \otimes_{A} \tau_{!}\left(N_{1}\right) \rightarrow$ $M \otimes_{A} \tau_{!}\left(N_{2}\right)$ est $\alpha$-nul. Comme le foncteur $\widetilde{\alpha}_{A}$ est exact et commute avec les produits tensoriels, le morphisme $\operatorname{id}_{M^{\alpha}} \otimes f: M^{\alpha} \otimes_{A^{\alpha}} N_{1} \rightarrow M^{\alpha} \otimes_{A^{\alpha}} N_{2}$ est une injection; d'où la platitude de $M^{\alpha}$.

D'autre part, supposons que $M^{\alpha}$ soit un $A^{\alpha}$-module plat et soit $f: N_{1} \rightarrow N_{2}$ une injection de $A$-modules. Posons $N=\operatorname{Ker}\left(M \otimes_{A} N_{1} \rightarrow M \otimes_{A} N_{2}\right)$. Comme le foncteur $\widetilde{\alpha}_{A}$ est exact et $M^{\alpha}$ plat, on a une injection de $A^{\alpha}$-modules $M^{\alpha} \otimes_{A^{\alpha}} N_{1}^{\alpha} \rightarrow$ $M^{\alpha} \otimes_{A^{\alpha}} N_{2}^{\alpha}$. On en déduit que $N^{\alpha}=0$; d'où l'assertion recherchée.

(ii) La suffisance de la condition résulte de (i) et de l'isomorphisme $N \stackrel{\sim}{\rightarrow}$ $\left(\widetilde{\tau}_{!}(N)\right)^{\alpha}(12.15($ iv $))$.

Supposons que $N$ soit un $A^{\alpha}$-module plat. En vertu de (i) et de l'isomorphisme $N \stackrel{\sim}{\rightarrow}\left(\widetilde{\tau}_{*}(N)\right)^{\alpha}(12.15($ ii)$)), \widetilde{\tau}_{*}(N)$ est $\alpha$-plat. Soit $f: N_{1} \rightarrow N_{2}$ un morphisme injectif de $A$-modules. Posons $L=\operatorname{Ker}\left(\widetilde{\tau}_{*}(N) \otimes_{A^{\alpha}} N_{1} \rightarrow \widetilde{\tau}_{*}(N) \otimes_{A^{\alpha}} N_{2}\right)$ qui est $\alpha$-nul. Compte tenu de la platitude de $\mathfrak{m}_{\tilde{\mathscr{C}}}$ sur $\mathfrak{o}_{\tilde{\mathscr{C}}}$, on a m $\mathfrak{m}_{\tilde{\mathscr{C}}} \otimes_{\mathfrak{o}_{\widetilde{\mathscr{C}}}} L=\operatorname{Ker}\left(\widetilde{\tau}_{!}(N) \otimes_{A^{\alpha}}\right.$ $\left.N_{1} \rightarrow \widetilde{\tau}_{!}(N) \otimes_{A^{\alpha}} N_{2}\right)$. Ce dernier est nul ; d'où la platitude de $\widetilde{\tau}_{!}(N)$.

LEMME 12.31. Conservons les notations de 12.25.

(i) Pour tout A-module plat $M, \varphi^{*}(M)$ est un $A^{\prime}$-module plat.

(ii) Pour tout $A^{\alpha}$-module plat $N, \psi(N)$ est un $A^{\prime \alpha}$-module plat.

Preuve. L'assertion (i) est démontrée dans [6, V 1.7.1]. D'après 12.30(ii), le $A$ module $\widetilde{\tau}_{!}(N)$ est plat. En vertu de (i), le $A^{\prime}$-module $\varphi^{*}\left(\widetilde{\tau}_{A^{!}}(N)\right) \simeq \widetilde{\tau}_{A^{\prime} !}(\psi(N))$ (12.25.4) est plat. La $A^{\alpha}$-platitude de $\psi(N)$ s'ensuit compte tenu de 12.30(ii).

12.32. On note $\widetilde{\mathscr{C}}^{\mathbb{N}^{\circ}}$ le topos des systèmes projectifs d'objets de $\widetilde{\mathscr{C}}(2.11)$. On pose $\mathfrak{o}_{\widetilde{\mathscr{C}}^{\mathbb{N}}}=\left(\mathfrak{o}_{\widetilde{\mathscr{C}}}\right)_{n \geqslant 1}\left(\right.$ resp. $\left.\mathfrak{m}_{\widetilde{\mathscr{C}}^{\mathrm{N}}}=\left(\mathfrak{m}_{\widetilde{\mathscr{C}}}\right)_{n \geqslant 1}\right)$ qui est isomorphe au faisceau associé au préfaisceau constant de valeur o (resp. $\mathfrak{m}$ ) de $\widetilde{\mathscr{C}}^{\mathbb{N}^{\circ}}$. Soit $\breve{A}=\left(A_{n}\right)_{n \geqslant 1}$ une $\mathfrak{o}_{\widetilde{C}^{\text {No }}}$-algèbre. On note $\operatorname{Mod}(\breve{A})$ la catégorie des $\breve{A}$-modules de $\widetilde{\mathscr{C}}^{\mathbb{N}^{\circ}}$.

Proposition 12.33. (i) Soit $M$ un A-module de $\widetilde{\mathscr{C}}$. On a un isomorphisme canonique fonctoriel (12.12.1)

$$
\widetilde{\tau}_{*}\left(M^{\alpha}\right) \stackrel{\sim}{\rightarrow} \mathscr{H}_{o m_{\mathfrak{o}_{\tilde{G}}}}\left(\mathfrak{m}_{\tilde{\mathscr{C}}}, M\right)
$$


(ii) Soit $M=\left(M_{n}\right)_{n \geqslant 1}$ un $\breve{A}$-module de $\widetilde{\widetilde{C}^{N^{0}}}$. On a un isomorphisme canonique fonctoriel

$$
\mathscr{H}_{o m_{\mathfrak{o}^{\mathbb{N}}}}\left(\mathfrak{m}_{\widetilde{\mathscr{C}} \mathbb{N}^{\circ}}, M\right) \simeq\left(\mathscr{H}_{o m_{\mathfrak{o}_{\widetilde{G}}}}\left(\mathfrak{m}_{\tilde{\mathscr{C}}}, M_{n}\right)\right)_{n \geqslant 1}
$$

Preuve. Soit $U$ un objet de $\widetilde{\mathscr{C}}$ et $j_{U}: \widetilde{\mathscr{C}}_{/ U} \rightarrow \widetilde{\mathscr{C}}$ le morphisme de localisation de $\tilde{\mathscr{C}}$ en $U$.

(i) On a un isomorphisme canonique fonctoriel $M(U) \stackrel{\sim}{\rightarrow} \Gamma\left(\widetilde{\mathscr{C}}_{/ U}, j_{U}^{*}(M)\right)$. Comme $\widehat{\alpha}$ et $\widehat{\sigma}_{*}$ transforment les faisceaux en des faisceaux $(12.7,12.8)$, d'après (12.1.5), on a un isomorphisme canonique fonctoriel

$$
\tilde{\tau}_{*}\left(M^{\alpha}\right)(U) \simeq \operatorname{Hom}_{\mathfrak{o}}(\mathfrak{m}, M(U)) .
$$

Par adjonction [6, IV 13.4.1], on a un isomorphisme canonique fonctoriel

$$
\operatorname{Hom}_{\mathfrak{o}}\left(\mathfrak{m}, \Gamma\left(\tilde{\mathscr{C}}_{/ U}, j_{U}^{*}(M)\right)\right) \simeq \operatorname{Hom}_{\mathfrak{o}_{\tilde{\mathscr{C}} \mid U}}\left(j_{U}^{*}\left(\mathfrak{m}_{\tilde{\mathscr{C}}}\right), j_{U}^{*}(M)\right) .
$$

L'assertion s'ensuit.

(ii) Soit $n$ un entier $\geqslant 1$. On désigne par $\alpha_{n}: \widetilde{\mathscr{C}} \rightarrow \widetilde{\mathscr{C}}^{\mathbb{N}^{\circ}}$ le morphisme de topos canonique défini pour tout faisceau $F=\left(F_{n}\right)_{n \geqslant 1}$ de $\widetilde{\mathscr{C}}^{\mathbb{N}^{\circ}} \operatorname{par} \alpha_{n}^{*}(F)=F_{n}$ [4, (III.7.1.2)]. Le foncteur $\alpha_{n}^{*}$ admet un adjoint à droit $\alpha_{n !}$ (cf. [4, (III.7.1.5)]). On en déduit des isomorphismes :

$$
\begin{aligned}
& \alpha_{n}^{*}\left(\mathscr{H}_{o m_{\mathfrak{G}^{\mathbb{N}}}}\left(\mathfrak{m}_{\widetilde{\mathscr{C}} \mathfrak{N N}^{\circ}}, M\right)\right)(U) \simeq \mathscr{H}_{o m_{\mathfrak{o}_{\widetilde{G}} \mathbb{N}^{0}}}\left(\mathfrak{m}_{\widetilde{\mathscr{C}}^{\mathbb{N}^{\circ}}}, M\right)\left(\alpha_{n !}(U)\right) \\
& \simeq \operatorname{Hom}_{\left.\mathfrak{o}_{\widetilde{G} \mathfrak{N}}\right|_{\alpha_{n !}(U)}}\left(\left.\mathfrak{m}_{\widetilde{\mathscr{C}} \mathbb{N}^{\mathrm{N}}}\right|_{\alpha_{n !}(U)},\left.M\right|_{\alpha_{n !}(U)}\right) \text {. }
\end{aligned}
$$

On note $[n]$ le sous-ensemble ordonné $\{1,2 \ldots, n\}$ de $\mathbb{N}$. On munit $\widetilde{\mathscr{C}}_{/ U} \times[n]$ de la topologie totale relative au site fibré constant $\tilde{\mathscr{C}}_{/ U} \times[n] \rightarrow[n]$ de fibre $\widetilde{\mathscr{C}}\left[4\right.$, VI.7.1] et on désigne par $\left(\widetilde{\mathscr{C}}_{/ U}\right)^{[n]^{\circ}}$ le topos des faisceaux d'ensembles sur $\tilde{\mathscr{C}}_{/ U} \times[n]$ (cf. [4, III.7.1]). Le foncteur d'injection canonique $\widetilde{\mathscr{C}}_{/ U} \times[n] \rightarrow \widetilde{\mathscr{C}}_{/ U} \times \mathbb{N}$ induit un morphisme de topos [4, III.7.8]

$$
\varphi_{n}:\left(\widetilde{\mathscr{C}}_{/ U}\right)^{[n]^{\circ}} \rightarrow\left(\widetilde{\mathscr{C}}_{/ U}\right)^{\mathbb{N}^{\circ}} .
$$

On note $j_{(U, n)}:\left(\widetilde{\mathscr{C}}^{\mathbb{N}^{\circ}}\right)_{\left./ \alpha_{n !} ! U\right)} \rightarrow \widetilde{\mathscr{C}}^{\mathbb{N}^{\circ}}$ le morphisme de localisation de $\widetilde{\mathscr{C}}^{\mathbb{N}^{\circ}}$ en $\alpha_{n !}(U)$. D'après [4, III.7.9], on a une équivalence canonique de topos

$$
h:\left(\tilde{\mathscr{C}}_{/ U}\right)^{[n]^{\circ}} \stackrel{\sim}{\rightarrow}\left(\widetilde{\mathscr{C}}^{\mathbb{N}^{\circ}}\right)_{/ \alpha_{n !}(U)}
$$

telle que $j_{(U, n)} \circ h$ soit le composé

$$
\left(\tilde{\mathscr{C}}_{/ U}\right)^{[n]^{\circ}} \stackrel{\varphi_{n}}{\rightarrow}\left(\tilde{\mathscr{C}}_{/ U}\right)^{\mathbb{N}^{\circ}} \stackrel{\left(j_{U}\right)^{\mathbb{N}^{\circ}}}{\longrightarrow} \widetilde{\mathscr{C}}^{\mathbb{N}^{\circ}}
$$


On en déduit un isomorphisme canonique fonctoriel

$$
\begin{aligned}
& \operatorname{Hom}_{\left.\mathfrak{o}_{\widetilde{\mathscr{G}} \mathbb{N}^{\circ}}\right|_{\alpha_{n !}(U)}}\left(\left.\mathfrak{m}_{\widetilde{\mathscr{C}}^{\mathbb{N}}}\right|_{\alpha_{n !}(U)},\left.M\right|_{\alpha_{n !}(U)}\right) \\
& \stackrel{\sim}{\rightarrow} \operatorname{Hom}_{\mathfrak{o}_{(\widetilde{\mathscr{C}} / U)}[n]^{\circ}}\left(\mathfrak{m}_{\left(\widetilde{\mathscr{C}}_{/ U}\right)^{[n]^{\circ}}},\left(j_{U}^{*}\left(M_{i}\right)\right)_{1 \leqslant i \leqslant n}\right) .
\end{aligned}
$$

D'après [4, VI.7.13], on a un isomorphisme canonique fonctoriel

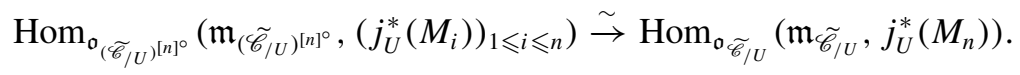

Compte tenu de (12.33.2), (12.33.3) et (12.33.4), on en déduit un isomorphisme canonique fonctoriel

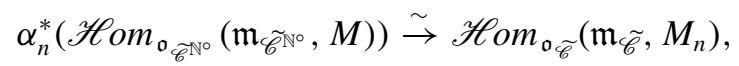

d'où la proposition.

12.34. Conservons les notations de 12.32 . On désigne par $\alpha-\widetilde{\mathscr{C}}^{\mathbb{N}^{\circ}}$ la catégorie des faisceaux de $\alpha$-o-modules de $\widetilde{\mathscr{C}}^{\mathbb{N}^{\circ}}$ (12.7). Pour tout entier $n \geqslant 1$, on désigne par $\operatorname{Mod}\left(A_{n}^{\alpha}\right)$ la catégorie des $A_{n}^{\alpha}$-modules de $\alpha-\widetilde{\mathscr{C}}$ et par $\operatorname{Mod}\left(\breve{A}^{\alpha}\right)$ la catégorie des $\breve{A}^{\alpha}$-modules de $\alpha-\widetilde{\mathscr{C}}^{\mathbb{N}^{\circ}}$. On note $\mathbf{P}\left(\operatorname{Mod}\left(A_{\bullet}^{\alpha}\right)\right)$ la catégorie des systèmes projectifs $\left(M_{n}\right)_{n \geqslant 1}$, où $M_{n}$ est un $A_{n}^{\alpha}$-module et $M_{n+1} \rightarrow M_{n}$ un morphisme $A_{n+1}^{\alpha}$-linéaire.

Soit $M=\left(M_{n}\right)_{n \geqslant 1}$ un objet de $\operatorname{Mod}(\breve{A})$ et $f=\left(f_{n}\right)_{n \geqslant 1}$ un morphisme de $\operatorname{Mod}(\breve{A})$. Pour que $M$ soit $\alpha$-nul, il faut et il suffit que, pour tout $n \geqslant 1, M_{n}$ soit $\alpha$ nul dans la catégorie $\operatorname{Mod}\left(A_{n}\right)$. Par suite, le morphisme $f$ est un $\alpha$-isomorphisme si et seulement si, pour tout entier $n \geqslant 1$, le morphisme $f_{n}$ est un $\alpha$-isomorphisme $\operatorname{de} \operatorname{Mod}\left(A_{n}\right)$. Par suite, le foncteur

$$
a: \operatorname{Mod}(\breve{A}) \rightarrow \mathbf{P}\left(\operatorname{Mod}\left(A_{\bullet}^{\alpha}\right)\right) \quad\left(M_{n}\right) \mapsto\left(M_{n}^{\alpha}\right)
$$

envoie les $\alpha$-isomorphismes sur des isomorphismes. Il induit donc un foncteur

$$
b: \operatorname{Mod}\left(\breve{A}^{\alpha}\right) \rightarrow \mathbf{P}\left(\operatorname{Mod}\left(A_{\bullet}^{\alpha}\right)\right) .
$$

Les foncteurs $\left(\widetilde{\tau}_{A_{n} *}: \operatorname{Mod}\left(A_{n}^{\alpha}\right) \rightarrow \operatorname{Mod}\left(A_{n}\right)\right)_{n \geqslant 1}(12.12 .1)$ induisent un foncteur

$$
t: \mathbf{P}\left(\operatorname{Mod}\left(A_{\bullet}^{\alpha}\right)\right) \rightarrow \operatorname{Mod}(\breve{A}) \quad\left(M_{n}\right) \mapsto\left(\tilde{\tau}_{*}\left(M_{n}\right)\right) .
$$

On désigne par

$$
s: \mathbf{P}\left(\operatorname{Mod}\left(A_{\bullet}^{\alpha}\right)\right) \rightarrow \operatorname{Mod}\left(\breve{A}^{\alpha}\right)
$$

le composé du foncteur $t$ et du foncteur canonique $\widetilde{\alpha}_{\breve{A}}: \operatorname{Mod}(\breve{A}) \rightarrow \operatorname{Mod}\left(\breve{A}^{\alpha}\right)$. Les isomorphismes $\widetilde{\alpha}_{A_{n}} \circ \tilde{\tau}_{A_{n} *} \stackrel{\sim}{\rightarrow} \mathrm{id}(12.15(\mathrm{ii}))$ induisent un isomorphisme

$$
b \circ s \stackrel{\sim}{\rightarrow} \mathrm{id} .
$$


D'après 12.33 , le foncteur composé

$$
t \circ a: \operatorname{Mod}(\breve{A}) \rightarrow \operatorname{Mod}(\breve{A})
$$

s'identifie au foncteur composé $\widetilde{\tau}_{\breve{A} *} \circ \widetilde{\alpha}_{\breve{A}}$. En vertu de 12.16(iii), le foncteur $\widetilde{\alpha}_{\breve{A}}$ : $\operatorname{Mod}(\breve{A}) \rightarrow \operatorname{Mod}\left(\breve{A}^{\alpha}\right)$ est isomorphe au foncteur composé

$$
\operatorname{Mod}(\breve{A}) \stackrel{a}{\rightarrow} \mathbf{P}\left(\operatorname{Mod}\left(A_{\bullet}^{\alpha}\right)\right) \stackrel{t}{\rightarrow} \operatorname{Mod}(\breve{A}) \stackrel{\widetilde{\alpha}_{\breve{A}}}{\rightarrow} \operatorname{Mod}\left(\breve{A}^{\alpha}\right)
$$

On en déduit un isomorphisme

$$
\mathrm{id} \stackrel{\sim}{\rightarrow} s \circ b .
$$

Par suite, $b$ et $s$ sont des équivalences de catégories, quasi inverses l'une de l'autre.

\section{Déformations des faisceaux de $\alpha$-modules}

Dans cette section, on se donne un topos $T$. On considère toujours $T$ comme muni de sa topologie canonique [6, II 2.5], qui en fait un site.

13.1. Rappelons d'abord la théorie des déformations pour les modules sur un topos annelé suivant [27, IV 3]. Soit

$$
p: A \rightarrow A_{0}
$$

une surjection de o-algèbres de $T$ dont le noyau $I$ est de carré nul. Soit $M_{0}, J$ deux $A_{0}$-modules et

$$
\tilde{M}=\left(0 \rightarrow J \rightarrow M \rightarrow M_{0} \rightarrow 0\right)
$$

une extension de $A$-modules. On a alors un diagramme commutatif

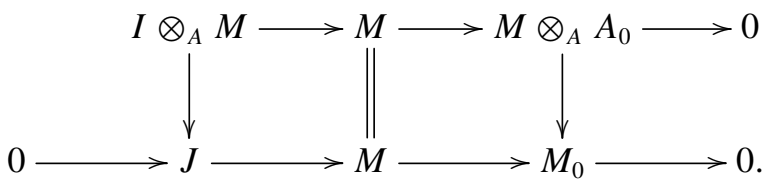

La flèche verticale de gauche induit un morphisme de $A_{0}$-modules

$$
u(\tilde{M}): I \otimes_{A_{0}} M_{0} \rightarrow J .
$$


En vertu de (13.1.2), $u(\widetilde{M})$ est un épimorphisme si et seulement si le morphisme canonique $M \otimes_{A} A_{0} \rightarrow M_{0}$ est un isomorphisme. La correspondance $\widetilde{M} \mapsto u(\widetilde{M})$ définit un homomorphisme (cf. [27, IV 3.1.1])

$$
u: \operatorname{Ext}_{A}^{1}\left(M_{0}, J\right) \rightarrow \operatorname{Hom}_{A_{0}}\left(I \otimes_{A_{0}} M_{0}, J\right) .
$$

On a une suite exacte (cf. [27, IV 3.1.4]) :

$$
\begin{aligned}
0 & \rightarrow \operatorname{Ext}_{A_{0}}^{1}\left(M_{0}, J\right) \rightarrow \operatorname{Ext}_{A}^{1}\left(M_{0}, J\right) \\
& \stackrel{u}{\rightarrow} \operatorname{Hom}_{A_{0}}\left(I \otimes_{A_{0}} M_{0}, J\right) \stackrel{\partial}{\rightarrow} \operatorname{Ext}_{A_{0}}^{2}\left(M_{0}, J\right),
\end{aligned}
$$

où la première flèche est le morphisme défini par restriction des scalaires.

ThÉORÈme 13.2 [27, IV 3.1.5]. Soit $M_{0}, J$ des $A_{0}$-modules et $u_{0}: I \otimes_{A_{0}} M_{0} \rightarrow J$ un morphisme $A_{0}$-linéaire. Alors:

(i) il existe une obstruction

$$
\partial\left(u_{0}\right) \in \operatorname{Ext}_{A_{0}}^{2}\left(M_{0}, J\right)
$$

dont l'annulation est nécessaire et suffisante pour l'existence d'une extension de A-modules $\tilde{M}$ de $M_{0}$ par J telle que $u(\widetilde{M})=u_{0}(13.1 .4)$;

(ii) lorsque $\partial\left(u_{0}\right)=0$, l'ensemble des classes d'isomorphismes de telles extensions $\widetilde{M}$ est un torseur sous $\operatorname{Ext}_{A_{0}}^{1}\left(M_{0}, J\right)$.

LEMME 13.3 [27, IV 3.1.1]. Supposons que le topos $T$ ait suffisamment de points. Soit $\widetilde{M}=\left(0 \rightarrow J \rightarrow M \rightarrow M_{0} \rightarrow 0\right)$ une extension de A-modules telle que le morphisme canonique $M \otimes_{A} A_{0} \rightarrow M_{0}$ soit un isomorphisme. Alors, pour que $M$ soit plat sur $A$, il faut et il suffit que $M_{0}$ soit plat sur $A_{0}$ et que $u(\widetilde{M})(13.1 .3)$ soit un isomorphisme.

Preuve. Comme $T$ a suffisamment de points, on peut se ramener au cas où $T$ est le topos ponctuel. L'assertion résulte alors du critère de platitude [24, 0.10.2.1].

REMARQUe 13.4. La suite exacte (13.1.5) peut s'établir par un calcul direct (cf. [27, IV 3.1.12]). L'isomorphisme de Cartan

$$
\mathbf{R H o m}_{A}\left(M_{0}, J\right) \stackrel{\sim}{\rightarrow} \mathbf{R H o m}_{A_{0}}\left(M_{0} \otimes_{A}^{\mathrm{L}} A_{0}, J\right)
$$

induit une suite spectrale

$$
\mathrm{E}_{2}^{i, j}=\operatorname{Ext}_{A_{0}}^{i}\left(\mathscr{T}_{o r}^{A}\left(M_{0}, A_{0}\right), J\right) \Rightarrow \mathrm{E}^{i+j}=\operatorname{Ext}_{A}^{i+j}\left(M_{0}, J\right) .
$$


Comme $M_{0}$ est un $A_{0}$-module, on a alors un isomorphisme canonique $\mathscr{T}_{0} r_{1}^{A}\left(M_{0}\right.$, $\left.A_{0}\right) \stackrel{\sim}{\rightarrow} I \otimes_{A_{0}} M$. La suite exacte des termes de bas degré de (13.4.2) fournit la suite exacte (13.1.5) (cf. [27, IV 3.1.13]).

13.5. Soit $\varphi:\left(T^{\prime}, A^{\prime}\right) \rightarrow(T, A)$ un morphisme plat de topos annelés [6, $\left.\mathrm{V} 1.8\right]$. On pose $A_{0}^{\prime}=\varphi^{*}\left(A_{0}\right)$ et $I^{\prime}=\varphi^{*}(I)$. On désigne par

$$
\begin{aligned}
\mathrm{E}_{2}^{i, j} & =\operatorname{Ext}_{A_{0}^{\prime}}^{i}\left(\mathscr{T}_{o r}^{A_{j}^{\prime}}\left(\varphi^{*}\left(M_{0}\right), A_{0}^{\prime}\right), \varphi^{*}(J)\right) \Rightarrow \\
\mathrm{E}^{\prime i+j} & =\operatorname{Ext}_{A^{\prime}}^{i+j}\left(\varphi^{*}\left(M_{0}\right), \varphi^{*}(J)\right)
\end{aligned}
$$

la suite spectrale (13.4.2) associée aux $A_{0}^{\prime}$-modules $\varphi^{*}\left(M_{0}\right)$ et $\varphi^{*}(J)$. Le faisceau $\mathscr{T}_{o r}^{j}\left(M_{0}, A_{0}\right)$ est calculé par une résolution plate de $M_{0}$. En vertu de 12.31(i) et de l'exactitude du foncteur $\varphi^{*}$, on a un isomorphisme canonique

$$
\varphi^{*}\left(\mathscr{T}_{\text {or }}^{j}\left(M_{0}, A_{0}\right)\right) \simeq \mathscr{T}_{A_{A^{\prime}}^{j}}^{j}\left(\varphi^{*}\left(M_{0}\right), A_{0}^{\prime}\right) .
$$

Le foncteur exact $\varphi^{*}$ induit un morphisme de suites spectrales (cf. 15.7)

$$
u: \mathrm{E}=\left(\mathrm{E}_{2}^{i, j}, \mathrm{E}^{n}\right) \rightarrow \mathrm{E}^{\prime}=\left(\mathrm{E}_{2}^{\prime i, j}, \mathrm{E}^{\prime n}\right)
$$

dont les morphismes

$$
\begin{aligned}
& u_{2}^{i, j}: \operatorname{Ext}_{A_{0}}^{i}\left(\mathscr{T o r}_{j}^{A}\left(M_{0}, A_{0}\right), J\right) \rightarrow \operatorname{Ext}_{A_{0}^{\prime}}^{i}\left(\mathscr{T o r}_{j}^{A^{\prime}}\left(\varphi^{*}\left(M_{0}\right), A_{0}^{\prime}\right), \varphi^{*}(J)\right), \\
& u^{n}: \operatorname{Ext}_{A}^{n}\left(M_{0}, J\right) \rightarrow \operatorname{Ext}_{A^{\prime}}^{n}\left(\varphi^{*}\left(M_{0}\right), \varphi^{*}(J)\right)
\end{aligned}
$$

s'identifient aux morphismes canoniques induits $\operatorname{par} \varphi^{*}$ (2.3.2). Les termes de bas degré du morphisme $u$ induisent un diagramme commutatif

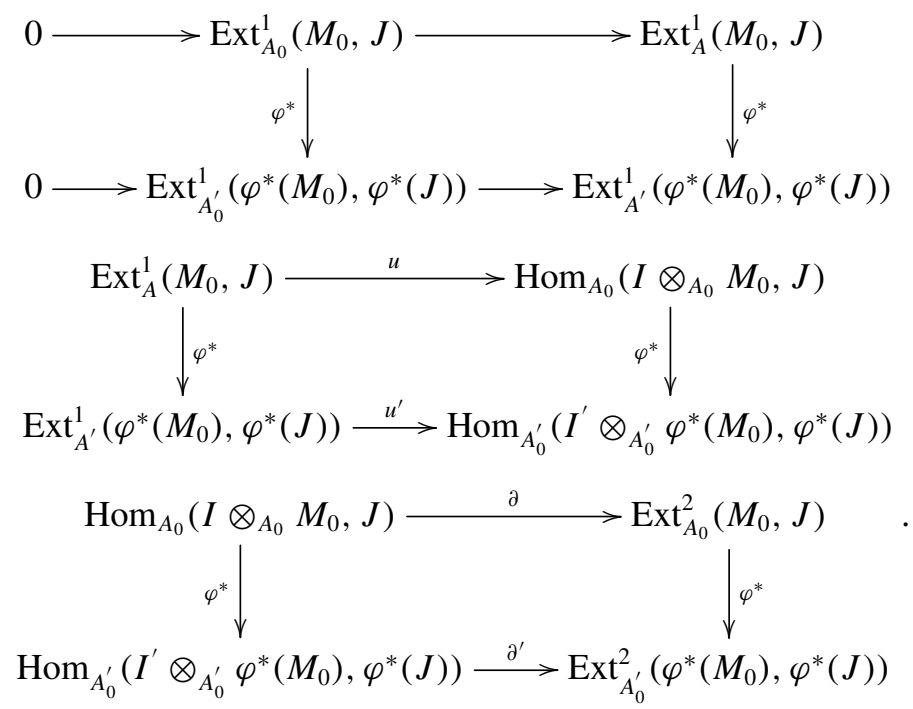


Cela signifie que la théorie de la déformation est fonctorielle par rapport au morphisme plat $\varphi$.

13.6. On note $\alpha$-T la catégorie des $\alpha$-o-modules de $T$ (12.7) et l'on pose $A_{0}^{\alpha}=$ $\widetilde{\alpha}\left(A_{0}\right)$ et $A^{\alpha}=\widetilde{\alpha}(A)$ (12.10.1). Soit $M_{0}$ et $J$ deux $A_{0}^{\alpha}$-modules. D'après 12.15(iv), (12.20.1) et (12.23.1), on a des isomorphismes canoniques

$$
\begin{aligned}
& \operatorname{Ext}_{A}^{1}\left(\widetilde{\tau}_{!}\left(M_{0}\right), \tilde{\tau}_{!}(J)\right) \stackrel{\sim}{\rightarrow} \operatorname{Ext}_{A^{\alpha}}^{1}\left(M_{0}, J\right), \\
& \operatorname{Ext}_{A_{0}}^{2}\left(\widetilde{\tau}_{!}\left(M_{0}\right), \widetilde{\tau}_{!}(J)\right) \stackrel{\sim}{\rightarrow} \operatorname{Ext}_{A_{0}^{\alpha}}^{2}\left(M_{0}, J\right), \\
& \operatorname{Hom}_{A_{0}}\left(I \otimes_{A_{0}} \widetilde{\tau}_{!}\left(M_{0}\right), \widetilde{\tau}_{!}(J)\right) \stackrel{\sim}{\rightarrow} \operatorname{Hom}_{A_{0}^{\alpha}}\left(I^{\alpha} \otimes_{A_{0}^{\alpha}} M_{0}, J\right) .
\end{aligned}
$$

On définit des morphismes

$$
\begin{gathered}
u^{\alpha}: \operatorname{Ext}_{A^{\alpha}}^{1}\left(M_{0}, J\right) \rightarrow \operatorname{Hom}_{A_{0}^{\alpha}}\left(I^{\alpha} \otimes_{A_{0}^{\alpha}} M_{0}, J\right) \\
\partial^{\alpha}: \operatorname{Hom}_{A_{0}^{\alpha}}\left(I^{\alpha} \otimes_{A^{\alpha}} M_{0}, J\right) \rightarrow \operatorname{Ext}_{A_{0}^{\alpha}}^{2}\left(M_{0}, J\right),
\end{gathered}
$$

par les morphismes $u$ et $\partial$ relatifs aux $A_{0}$-modules $\widetilde{\tau}_{!}\left(M_{0}\right)$ et $\widetilde{\tau}_{!}(J)$ (13.1.5). On en déduit par (13.1.5) une suite exacte

$$
\begin{aligned}
0 & \rightarrow \operatorname{Ext}_{A_{0}^{\alpha}}^{1}\left(M_{0}, J\right) \rightarrow \operatorname{Ext}_{A^{\alpha}}^{1}\left(M_{0}, J\right) \\
& \stackrel{u^{\alpha}}{\rightarrow} \operatorname{Hom}_{A_{0}^{\alpha}}\left(I^{\alpha} \otimes_{A_{0}^{\alpha}} M_{0}, J\right) \stackrel{\partial^{\alpha}}{\rightarrow} \operatorname{Ext}_{A_{0}^{\alpha}}^{2}\left(M_{0}, J\right),
\end{aligned}
$$

où la première flèche est le morphisme défini par restriction des scalaires.

ThÉORÈme 13.7. Conservons les notations de 13.6. Soit $M_{0}, J$ des $A_{0}^{\alpha}$-modules et $u_{0}: I^{\alpha} \otimes_{A_{0}^{\alpha}} M_{0} \rightarrow J$ un morphisme $A_{0}^{\alpha}$-linéaire. Alors :

(i) il existe une obstruction

$$
\partial^{\alpha}\left(u_{0}\right) \in \operatorname{Ext}_{A_{0}^{\alpha}}^{2}\left(M_{0}, J\right)
$$

dont l'annulation est nécessaire et suffisante pour l'existence d'une extension de $A^{\alpha}$-modules $\widetilde{M}$ de $M_{0}$ par $J$ telle que $u^{\alpha}(\widetilde{M})=u_{0}(13.6 .1)$;

(ii) lorsque $\partial^{\alpha}\left(u_{0}\right)=0$, l'ensemble des classes d'isomorphismes de telles extensions $\widetilde{M}$ est un torseur sous $\operatorname{Ext}_{A_{0}^{\alpha}}^{1}\left(M_{0}, J\right)$.

13.8. Conservons les notations de 13.5. On note $\alpha-T^{\prime}$ la catégorie des faisceaux de $\alpha$-o-modules de $T^{\prime}(12.7)$ et l'on pose $A^{\prime \alpha}=\widetilde{\alpha}\left(A^{\prime}\right)$ et $A_{0}^{\prime \alpha}=\widetilde{\alpha}\left(A_{0}^{\prime}\right)(12.10 .1)$. Le foncteur exact $\varphi^{*}: \operatorname{Mod}(A) \rightarrow \operatorname{Mod}\left(A^{\prime}\right)$ s'étend en un foncteur exact $\psi:$ 
$\operatorname{Mod}\left(A^{\alpha}\right) \rightarrow \operatorname{Mod}\left(A^{\prime \alpha}\right)$ (12.26). D'après (12.26.1) et (13.5.2), on a un diagramme commutatif (2.5.3)

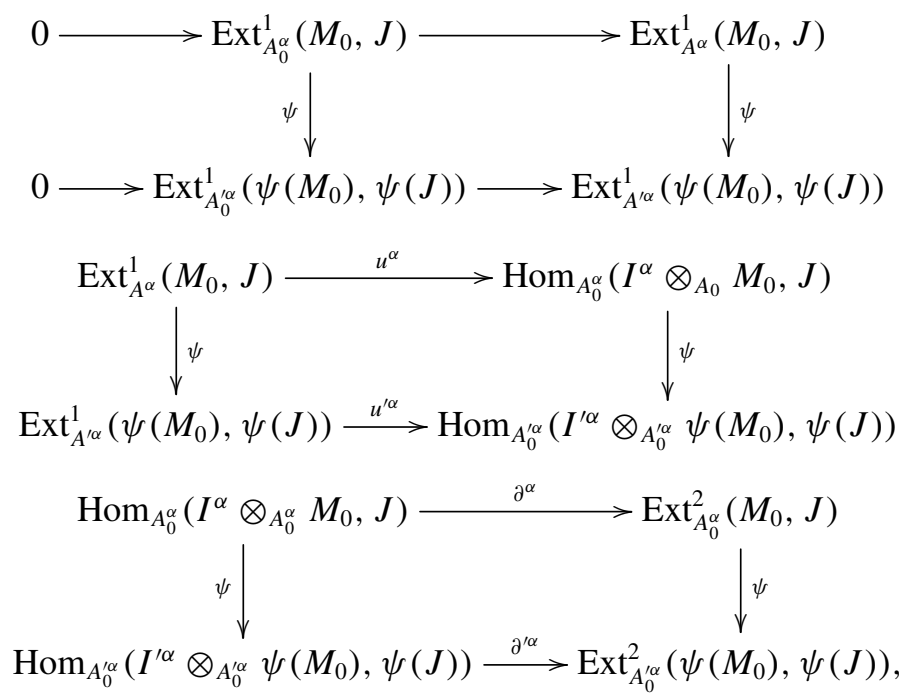

où les flèches horizontales sont induites par le foncteur exact $\psi$ (2.3.2). Cela signifie que la théorie de la déformation pour les $\alpha$-modules est fonctorielle par rapport au morphisme plat $\varphi$.

13.9. Soit $A$ une o-algèbre (ou $\mathcal{O}_{\bar{K}}$-algèbre) plate de $T$. Pour tout entier $n \geqslant 1$, on pose $A_{n}=A / p^{n} A$ et $I_{n}=p^{n} A / p^{2 n} A$ qui est un idéal de $A_{2 n}$. Le morphisme de multiplication par $p^{n}$ induit, pour tout entier $n \geqslant 1$, un isomorphisme canonique de $A_{n}$-modules $I_{n} \stackrel{\sim}{\rightarrow} A_{n}$. Pour tout entier $n \geqslant 1$, on pose $A_{n}^{\alpha}=\widetilde{\alpha}\left(A_{n}\right)(12.10 .1)$.

Soit $n$ un entier $\geqslant 1, M_{n}$ un $A_{n}$-module et $N_{n}$ un $A_{n}^{\alpha}$-module. L'isomorphisme $I_{n} \stackrel{\sim}{\rightarrow} A_{n}$ induit un isomorphisme canonique de $A_{n}$-modules (resp. $A_{n}^{\alpha}$-modules)

$$
v: I_{n} \otimes_{A_{n}} M_{n} \stackrel{\sim}{\rightarrow} M_{n} \quad\left(\text { resp. } \quad w: I_{n}^{\alpha} \otimes_{A_{n}^{\alpha}} N_{n} \stackrel{\sim}{\rightarrow} N_{n}\right)
$$

On appelle déformation de $M_{n}$ sur $A_{2 n}$ (resp. déformation de $N_{n}$ sur $A_{2 n}^{\alpha}$ ) toute extension de $A_{2 n}$-modules (resp. $A_{2 n}^{\alpha}$-modules)

$$
\begin{aligned}
\tilde{M} & =\left(0 \rightarrow M_{n} \rightarrow M_{2 n} \rightarrow M_{n} \rightarrow 0\right) \\
\text { (resp. } \quad \tilde{N} & \left.=\left(0 \rightarrow N_{n} \rightarrow N_{2 n} \rightarrow N_{n} \rightarrow 0\right)\right)
\end{aligned}
$$

telle que $u(\tilde{M})=v\left(\operatorname{resp} . u^{\alpha}(\tilde{N})=w\right)(13.9 .1)$. 
13.10. Pour toute déformation $0 \rightarrow M_{n} \rightarrow M_{2 n} \rightarrow M_{n} \rightarrow 0$ de $M_{n}$ sur $A_{2 n}$, il est clair que $0 \rightarrow M_{n}^{\alpha} \rightarrow M_{2 n}^{\alpha} \rightarrow M_{n}^{\alpha} \rightarrow 0$ est une déformation de $M_{n}^{\alpha}$ sur $A_{2 n}^{\alpha}$. D'autre part, si $0 \rightarrow N_{n} \rightarrow N_{2 n} \rightarrow N_{n} \rightarrow 0$ est une déformation de $N_{n}$ sur $A_{2 n}^{\alpha}$, la suite exacte $0 \rightarrow \widetilde{\tau}_{!}\left(N_{n}\right) \rightarrow \widetilde{\tau}_{!}\left(N_{2 n}\right) \rightarrow \widetilde{\tau}_{!}\left(N_{n}\right) \rightarrow 0$ est une déformation de $\widetilde{\tau}_{!}\left(N_{n}\right)$ sur $A_{2 n}$ en vertu de 12.16(i) et (12.23.1).

LEMME 13.11. Supposons que le topos $T$ ait suffisamment de points et soit un entier $n \geqslant 1$.

(i) Soit $M_{n}$ un $A_{n}$-module plat. Une déformation de $M_{n}$ sur $A_{2 n}$ est équivalente à la donnée d'un $A_{2 n}$-module plat $M_{2 n}$ et d'un épimorphisme $A_{2 n}$-linéaire $g: M_{2 n} \rightarrow M_{n}$ tels que le morphisme canonique $M_{2 n} \otimes_{A_{2 n}} A_{n} \rightarrow M_{n}$ soit un isomorphisme.

(ii) Soit $N_{n}$ un $A_{n}^{\alpha}$-module plat (12.29(ii)). Une déformation de $N_{n}$ sur $A_{2 n}^{\alpha}$ est équivalente à la donnée d'un $A_{2 n}^{\alpha}$-module plat $N_{2 n}$ et d'un épimorphisme $A_{2 n}^{\alpha}$ linéaire $g: N_{2 n} \rightarrow N_{n}$ tels que le morphisme canonique $N_{2 n} \otimes_{A_{2 n}^{\alpha}} A_{n}^{\alpha} \rightarrow N_{n}$ soit un isomorphisme.

Preuve. (i) Soit $0 \rightarrow M_{n} \rightarrow M_{2 n} \rightarrow M_{n} \rightarrow 0$ une déformation de $M_{n}$ sur $A_{2 n}$. Comme $v$ est un isomorphisme, le morphisme $M_{2 n} \otimes_{A_{2 n}} A_{n} \rightarrow M_{n}$ est un isomorphisme (cf. 13.1). D'après 13.3, $M_{2 n}$ est plat sur $A_{2 n}$.

D'autre part, soit $M_{2 n}$ un $A_{2 n}$-module plat et $g: M_{2 n} \rightarrow M_{n}$ un morphisme tel que $M_{2 n} \otimes_{A_{2 n}} A_{n} \stackrel{\sim}{\rightarrow} M_{n}$. Par platitude de $M_{2 n}$, on en déduit un isomorphisme $I_{n} \otimes_{A_{2 n}} M_{2 n} \stackrel{\sim}{\rightarrow} \operatorname{Ker}(g)$, d'où l'assertion.

(ii) Soit $0 \rightarrow N_{n} \rightarrow N_{2 n} \rightarrow N_{n} \rightarrow 0$ une déformation de $N_{n}$ sur $A_{2 n}^{\alpha}$. D'après 12.30(ii), $\widetilde{\tau}_{!}\left(N_{n}\right)$ est $A_{n}$-plat. En vertu de (i) et $13.10, \widetilde{\tau}_{!}\left(N_{2 n}\right)$ est $A_{2 n}$-plat et l'on a un isomorphisme $\widetilde{\tau}_{!}\left(N_{2 n}\right) \otimes_{A_{2 n}} A_{n} \simeq \widetilde{\tau}_{!}\left(N_{n}\right)$. On en déduit par 12.15(iv) un isomorphisme $N_{2 n} \otimes_{A_{2 n}^{\alpha}} A_{n}^{\alpha} \simeq N_{n}$. La platitude de $N_{2 n}$ résulte de 12.30(i).

D'autre part, soit $N_{2 n}$ un $A_{2 n}^{\alpha}$-module plat et $g: N_{2 n} \rightarrow N_{n}$ un morphisme tel que $N_{2 n} \otimes_{A_{2 n}^{\alpha}} A_{n}^{\alpha} \stackrel{\sim}{\rightarrow} N_{n}$. Par platitude de $N_{2 n}$, on en déduit un isomorphisme $I_{n}^{\alpha} \otimes_{A_{2 n}^{\alpha}} N_{2 n} \stackrel{\sim}{\rightarrow} \operatorname{Ker}(g)$, d'où l'assertion.

13.12. Dans la suite de cette section, on se donne un schéma connexe $X$ et un point géométrique $\bar{x}$ de $X$. Soit un entier $n \geqslant 1$. On note $X_{\text {fét }}$ le topos fini étale de $X$ et $\mathfrak{o}_{n}$ le faisceau constant de valeur $\mathfrak{o}_{n}$ de $X_{\text {fét. }}$. Le foncteur fibre en $\bar{x}$

$$
\operatorname{Mod}\left(X_{\text {fét }}, \mathfrak{o}_{n}\right) \rightarrow \operatorname{Mod}\left(\mathfrak{o}_{n}\right), \quad \mathbb{L} \mapsto \mathbb{L}_{\bar{x}}
$$

induit une équivalence de catégories (2.14.3)

$$
\operatorname{Mod}\left(X_{\text {fét }}, \mathfrak{o}_{n}\right) \stackrel{\sim}{\rightarrow} \operatorname{Rep}_{\mathfrak{o}_{n}}\left(\pi_{1}(X, \bar{x})\right),
$$


où le but désigne la catégorie des $\mathfrak{o}_{n}$-représentations de $\pi_{1}(X, \bar{x})$ (3.18). On désigne par $\operatorname{Mod}^{\alpha \text { ptf }}\left(X_{\text {fét }}, \mathfrak{o}_{n}\right)$ la catégorie des $\mathfrak{o}_{n}$-modules $\alpha$-plats de type $\alpha$-fini de $X_{\text {fét }}(12.28,12.29)$.

Lemme 13.13. (i) Pour qu'un $\mathfrak{o}_{n}$-module $\mathbb{L}$ de $X_{\text {fét }}$ soit $\alpha$-plat de type $\alpha$-fini, il faut et il suffit que sa fibre $\mathbb{L}_{\bar{x}}$ (13.12.1) soit un $\mathfrak{o}_{n}$-module $\alpha$-plat de type $\alpha$-fini.

(ii) Le foncteur (13.12.2) induit une équivalence de catégories (3.23)

$$
\operatorname{Mod}^{\alpha \mathrm{ptf}}\left(X_{\mathrm{fét}}, \mathfrak{o}_{n}\right) \stackrel{\sim}{\rightarrow} \operatorname{Rep}_{\mathfrak{o}_{n}}^{\alpha \mathrm{ptf}}\left(\pi_{1}(X, \bar{x})\right) .
$$

Preuve. (i) Comme le foncteur (13.12.1) est conservatif, la $\alpha$-platitude d'un $\mathfrak{o}_{n}$ module de $X_{\text {fét }}$ est équivalente à la $\alpha$-platitude de sa fibre en $\bar{x}$.

Si $\mathbb{L}$ est de type $\alpha$-fini, sa fibre $\mathbb{L}_{\bar{x}}$ est évidemment de type $\alpha$-fini. Supposons que $\mathbb{L}_{\bar{x}}$ soit de type $\alpha$-fini et montrons que $\mathbb{L}$ est de type $\alpha$-fini. Pour tout $\gamma \in \mathfrak{m}$, il existe un $\mathfrak{o}_{n}$-module libre de type fini $M$ et un morphisme $\mathfrak{o}_{n}$-linéaire $u: M \rightarrow \mathbb{L}_{\bar{x}}$ dont le noyau est annulé par $\gamma$. Soit $e_{1}, \ldots, e_{m}$ une base de $M$. L'assertion recherchée étant locale pour $X_{\text {fét }}$, quitte à remplacer $X$ par un revêtement étale, on peut supposer que $\pi_{1}(X, \bar{x})$ fixe les éléments $u\left(e_{1}\right), \ldots, u\left(e_{m}\right)$ de $\mathbb{L}_{\bar{x}}$. Munissant $M$ de l'unique $\mathfrak{o}_{n}$-représentation de $\pi_{1}(X, \bar{x})$ telle que $e_{1}, \ldots, e_{m}$ soient fixes, l'homomorphisme $u$ est alors $\pi_{1}(X, \bar{x})$-équivariant. Par suite, $\mathbb{L}$ est un $\mathfrak{o}_{n}$-module de type $\alpha$-fini de $X_{\text {fét }}$.

(ii) Cela résulte de (i).

13.14. On désigne par $\alpha$ - $X_{\text {fét }}$ la catégorie des $\alpha$-o-modules de $X_{\text {fét }}$ (12.7). Soit un entier $n \geqslant 1$. On pose $\mathfrak{o}_{n}^{\alpha}=\widetilde{\alpha}\left(\mathfrak{o}_{n}\right)(12.10 .1)$ et l'on désigne par $\operatorname{Mod}\left(X_{\text {fét }}, \mathfrak{o}_{n}^{\alpha}\right)$ la catégorie des $\mathfrak{o}_{n}^{\alpha}$-modules de $\alpha$ - $X_{\text {fét }}$ (12.11). On a un foncteur canonique (12.7.2)

$$
\widetilde{\alpha}: \operatorname{Mod}\left(X_{\text {fét }}, \mathfrak{o}_{n}\right) \rightarrow \operatorname{Mod}\left(X_{\text {fét }}, \mathfrak{o}_{n}^{\alpha}\right) \text {. }
$$

On désigne par $\operatorname{Mod}^{\mathrm{ptf}}\left(X_{\text {fét }}, \mathfrak{o}_{n}^{\alpha}\right)$ l'image essentielle de la catégorie $\operatorname{Mod}^{\alpha \mathrm{ptf}}\left(X_{\text {fét }}, \mathfrak{o}_{n}\right)$ dans $\operatorname{Mod}\left(X_{\text {fét }}, \mathfrak{o}_{n}^{\alpha}\right)$.

LEMME 13.15. Soit $\mathscr{L}$ un objet de $\operatorname{Mod}^{\mathrm{ptf}}\left(X_{\mathrm{fét}}, \mathfrak{o}_{n}^{\alpha}\right)$.

(i) Le $\mathfrak{o}_{n}^{\alpha}$-module $\mathscr{L}$ est plat (12.29).

(ii) Toute déformation de $\mathscr{L}$ sur $\mathfrak{o}_{2 n}^{\alpha}(13.11(\mathrm{ii}))$ est un objet de $\mathbf{M o d}^{\mathrm{ptf}}\left(X_{\text {fét }}, \mathfrak{o}_{2 n}^{\alpha}\right)$.

Preuve. (i) Cela résulte de 12.30(i). 
(ii) Soit $\mathbb{L}$ un $\mathfrak{o}_{n}$-module $\alpha$-plat de type $\alpha$-fini de $X_{\text {fét }}$ tel que $\mathbb{L}^{\alpha} \simeq \mathscr{L}$. On en déduit par adjonction (12.15(iii)) un $\alpha$-isomorphisme $\widetilde{\tau}_{!}(\mathscr{L}) \rightarrow \mathbb{L} ;$ d'où la $\alpha$ finitude de $\widetilde{\tau}_{!}(\mathscr{L})$. D'après $12.30\left(\right.$ ii), $\widetilde{\tau}_{!}(\mathscr{L})$ est $\mathfrak{o}_{n}$-plat. Soit $\mathscr{L}^{\prime}$ une déformation de $\mathscr{L}$ sur $\mathfrak{o}_{2 n}^{\alpha}$. En vertu de 13.10 et $13.11(\mathrm{i}), \widetilde{\tau}_{!}\left(\mathscr{L}^{\prime}\right)$ est une déformation de $\widetilde{\tau}_{!}(\mathscr{L})$ sur $\mathfrak{o}_{2 n}$ et est donc $\mathfrak{o}_{2 n}$-plat. Par ailleurs, on a une suite exacte de $\mathfrak{o}_{2 n}$-modules

$$
0 \rightarrow \widetilde{\tau}_{!}(\mathscr{L})_{\bar{x}} \rightarrow \widetilde{\tau}_{!}\left(\mathscr{L}^{\prime}\right)_{\bar{x}} \rightarrow \widetilde{\tau}_{!}(\mathscr{L})_{\bar{x}} \rightarrow 0 .
$$

On en déduit par 3.7 et 13.13(i) la $\alpha$-finitude de $\widetilde{\tau}_{!}\left(\mathscr{L}^{\prime}\right)$. Par suite, $\widetilde{\tau}_{!}\left(\mathscr{L}^{\prime}\right)$

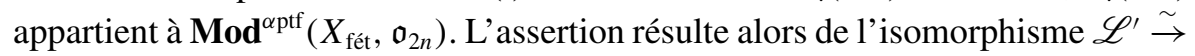
$\left(\widetilde{\tau}_{!}\left(\mathscr{L}^{\prime}\right)\right)^{\alpha}(12.15($ iv $))$.

13.16. On note $X_{\text {fét }}^{\mathbb{N} o}$ le topos des systèmes projectifs d'objets de $X_{\text {fét }}(2.11)$ et $\breve{\mathfrak{o}}$ l'anneau $\left(\mathfrak{o}_{n}\right)_{n \geqslant 1}$ de $X_{\text {fét }}^{\mathbb{N}^{\circ}}$. On désigne par $\operatorname{Mod}^{\alpha \text { ptf }}\left(X_{\text {fét }}^{\mathbb{N}^{\circ}}, \breve{\mathfrak{o}}\right)$ la sous-catégorie pleine $\operatorname{de} \operatorname{Mod}\left(X_{\text {fét }}^{\mathbb{N}^{\circ}}, \breve{\mathfrak{o}}\right)$ formée des $\breve{\mathfrak{o}}$-modules $\left(\mathbb{L}_{n}\right)_{n \geqslant 1}$ tels que, pour tout entier $n \geqslant 1$, $\mathbb{L}_{n}$ soit un objet de $\operatorname{Mod}^{\alpha \text { ptf }}\left(X_{\text {fét }}, \mathfrak{o}_{n}\right)$ et que le morphisme canonique $\mathbb{L}_{n+1} \otimes_{\mathfrak{o}_{n+1}}$ $\mathfrak{o}_{n} \rightarrow \mathbb{L}_{n}$ soit un $\alpha$-isomorphisme. Compte tenu de 3.23, les foncteurs (13.13.1) induisent une équivalence de catégories

$$
\operatorname{Mod}^{\alpha \mathrm{ptf}}\left(X_{\text {fét }}^{\mathbb{N}^{\circ}}, \breve{\mathfrak{o}}\right) \stackrel{\sim}{\rightarrow} \operatorname{Rep}_{\breve{\mathfrak{o}}}^{\alpha \mathrm{ptf}}\left(\pi_{1}(X, \bar{x})\right) .
$$

On désigne par $\mathbf{P}\left(\operatorname{Mod}\left(X_{\text {fét }}, \mathfrak{o}_{\bullet}^{\alpha}\right)\right)$ la catégorie des systèmes projectifs $\left(\mathscr{L}_{n}\right)_{n \geqslant 1}$, où $\mathscr{L}_{n}$ est un $\mathfrak{o}_{n}^{\alpha}$-module de $\alpha$ - $X_{\text {fét }}$ et $\mathscr{L}_{n+1} \rightarrow \mathscr{L}_{n}$ est un morphisme $\mathfrak{o}_{n+1}^{\alpha}$-linéaire (12.34). Rappelons que le foncteur canonique

$$
\operatorname{Mod}\left(X_{\text {fét }}^{\mathbb{N}^{\circ}}, \breve{\mathfrak{o}}\right) \rightarrow \mathbf{P}\left(\operatorname{Mod}\left(X_{\text {fét }}, \mathfrak{o}_{\bullet}^{\alpha}\right)\right) \quad\left(\mathbb{L}_{n}\right)_{n \geqslant 1} \mapsto\left(\mathbb{L}_{n}^{\alpha}\right)_{n \geqslant 1}
$$

induit une équivalence de catégories (12.34.2)

$$
\operatorname{Mod}\left(X_{\text {fét }}^{\mathbb{N}^{\circ}}, \breve{\mathfrak{o}}^{\alpha}\right) \stackrel{\sim}{\rightarrow} \mathbf{P}\left(\operatorname{Mod}\left(X_{\text {fét }}, \mathfrak{o}_{\bullet}^{\alpha}\right)\right)
$$

On désigne par $\mathbf{P}\left(\mathbf{M o d}^{\alpha \mathrm{ptf}}\left(X_{\text {fét }}, \mathfrak{o}_{\bullet}^{\alpha}\right)\right)$ la sous-catégorie pleine de $\mathbf{P}\left(\mathbf{M o d}\left(X_{\text {fét }}\right.\right.$, $\left.\mathfrak{o}_{\bullet}^{\alpha}\right)$ ) formée des systèmes projectifs $\left(\mathscr{L}_{n}\right)_{n \geqslant 1}$ tels que, pour tout entier $n \geqslant 1$, $\mathscr{L}_{n}$ soit un objet de $\operatorname{Mod}^{\text {ptf }}\left(X_{\text {fét }}, \mathfrak{o}_{n}^{\alpha}\right)$ (13.14) et que le morphisme canonique $\mathscr{L}_{n+1} \otimes_{\mathfrak{o}_{n+1}^{\alpha}} \mathfrak{o}_{n}^{\alpha} \stackrel{\sim}{\rightarrow} \mathscr{L}_{n}$ soit un isomorphisme. On note $\mathbf{P}\left(\mathbf{M o d}^{\mathrm{ptf}}\left(X_{\text {fét }}, \mathfrak{o}_{\bullet}^{\alpha}\right)\right)_{\mathbb{Q}}$ la catégorie des objets de $\mathbf{P}\left(\mathbf{M o d}^{\mathrm{ptf}}\left(X_{\text {fét }}, \mathfrak{o}_{\bullet}^{\alpha}\right)\right)$ à isogénie près. On désigne par $\mathbf{L L}^{\mathrm{tf}}\left(X_{\text {fét }}^{\mathbb{N}^{\circ}}, \breve{\mathfrak{o}}\right)\left(\right.$ resp. $\left.\mathbf{L} \mathbf{L}_{\mathbb{Q}}^{\mathrm{tf}}\left(X_{\text {fét }}^{\mathbb{N}^{\circ}}, \breve{\mathfrak{o}}\right)\right)$ la catégorie des $\breve{\mathfrak{o}}$-modules localement libres de type fini de $X_{\text {fét }}^{\mathbb{N}^{\circ}}$ (resp. $\breve{o}$-modules localement libres de type fini de $X_{\text {fét }}^{\mathbb{N}^{\circ}}$ à isogénie près).

LEMME 13.17. La catégorie $\mathbf{P}\left(\operatorname{Mod}^{\alpha \mathrm{ptf}}\left(X_{\text {fét }}, \mathfrak{o}_{\bullet}^{\alpha}\right)\right)$ est l'image essentielle de la

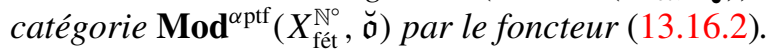


Preuve. Il est clair que l'image essentielle de $\operatorname{Mod}^{\alpha \mathrm{ptf}}\left(X_{\text {fét }}^{\mathbb{N}^{\circ}}, \breve{\mathfrak{o}}\right)$ est contenue dans $\mathbf{P}\left(\mathbf{M o d}^{\alpha \mathrm{ptf}}\left(X_{\text {fét }}, \mathfrak{o}_{\bullet}^{\alpha}\right)\right)$. Soit $\mathscr{L}=\left(\mathscr{L}_{n}\right)_{n \geqslant 1}$ un objet de $\mathbf{P}\left(\mathbf{M o d}^{\alpha p t f}\left(X_{\text {fét }}\right.\right.$, $\left.\left.\mathfrak{o}_{\bullet}^{\alpha}\right)\right)$. En vertu de la preuve de $13.15(\mathrm{ii}), \widetilde{\tau}_{!}\left(\mathscr{L}_{n}\right)$ est $\alpha$-plat de type $\alpha$-fini. L'isomorphisme canonique $\mathscr{L}_{n+1} \otimes_{\mathfrak{o}_{n+1}^{\alpha}} \mathfrak{o}_{n}^{\alpha} \stackrel{\sim}{\rightarrow} \mathscr{L}_{n}$ induit un isomorphisme $\tilde{\tau}_{!}(\mathscr{L})_{n+1} \quad \otimes_{\mathfrak{o}_{n+1}} \quad \mathfrak{o}_{n} \stackrel{\sim}{\rightarrow} \tilde{\tau}_{!}\left(\mathscr{L}_{n}\right) \quad$ (12.23.1). L'assertion résulte alors de l'isomorphisme $\left(\mathscr{L}_{n}\right)_{n \geqslant 1} \stackrel{\sim}{\rightarrow}\left(\left(\widetilde{\tau}_{!}\left(\mathscr{L}_{n}\right)\right)^{\alpha}\right)_{n \geqslant 1}(12.15(\mathrm{iv}))$.

LEMME 13.18. Le foncteur canonique

$$
\mathbf{L L}^{\mathrm{tf}}\left(X_{\text {fét }}^{\mathbb{N}^{\circ}}, \breve{\mathfrak{o}}\right) \rightarrow \mathbf{M o d}^{\alpha \mathrm{ptf}}\left(X_{\text {fét }}^{\mathbb{N}^{\circ}}, \breve{\mathfrak{o}}\right)
$$

induit une équivalence de catégories

$$
\mathbf{L L}_{\mathbb{Q}}^{\mathrm{tf}}\left(X_{\text {fét }}^{\mathbb{N}^{\circ}}, \breve{\mathfrak{o}}\right) \stackrel{\sim}{\rightarrow} \mathbf{P}\left(\mathbf{M o d}^{\mathrm{ptf}}\left(X_{\text {fét }}, \mathfrak{o}_{\bullet}^{\alpha}\right)\right)_{\mathbb{Q}}
$$

Preuve. D'après 3.26, 3.27, (3.29.4) et (13.16.1), le foncteur (13.18.1), qui correspond au foncteur canonique $\operatorname{Rep}_{\mathfrak{a}}^{\text {lff }}\left(\pi_{1}(X, \bar{x})\right) \rightarrow \operatorname{Rep}_{\mathfrak{a}}^{\alpha \text { ptf }}\left(\pi_{1}(X, \bar{x})\right)$, induit une équivalence de catégories $\mathbf{L L}_{\mathbb{Q}}^{\mathrm{tf}}\left(X_{\text {fét }}^{\mathbb{N}^{\circ}}, \breve{\mathfrak{o}}\right) \stackrel{\sim}{\rightarrow} \operatorname{Mod}_{\mathbb{Q}}^{\alpha \text { ptf }}\left(X_{\text {fét }}^{\mathbb{N}^{\circ}}, \breve{\mathfrak{o}}\right)$, où le but désigne la catégorie des objets de $\operatorname{Mod}^{\alpha p t f}\left(X_{\text {fét }}^{\mathbb{N}^{\circ}}, \breve{o}\right)$ à isogénie près. L'équivalence de catégories $\operatorname{Mod}_{\mathbb{Q}}^{\alpha \text { ptf }}\left(X_{\text {fét }}^{\mathbb{N} o}, \breve{o}\right) \simeq \mathbf{P}\left(\operatorname{Mod}^{\text {ptf }}\left(X_{\text {fét }}, \mathfrak{o}_{\bullet}^{\alpha}\right)\right)_{\mathbb{Q}}$ résulte de 12.22 et 13.17.

\section{Déformations des représentations et déformations des $\alpha$-modules de Faltings}

14.1. Soit $X$ un $S$-schéma propre à réduction semi-stable de fibre générique géométrique connexe et un entier $n \geqslant 1$. On note $(\widetilde{E}, \overline{\mathscr{B}})\left(\operatorname{resp} .\left(\widetilde{E}_{s}, \overline{\mathscr{B}}_{n}\right)\right)$ le topos annelé de Faltings (resp. la fibre spéciale du topos annelé de Faltings) associé au $S$-schéma $X$ (7.11). On désigne par $\alpha-X_{\bar{\eta} \text {,fét }}\left(\right.$ resp. $\left.\alpha-\widetilde{E}_{s}\right)$ la catégorie des $\alpha$-omodules de $X_{\bar{\eta} \text {,fét }}\left(\right.$ resp. $\left.\widetilde{E}_{s}\right)(12.7)$. On pose $\mathfrak{o}_{n}^{\alpha}=\widetilde{\alpha}\left(\mathfrak{o}_{n}\right), \overline{\mathscr{B}}_{n}^{\alpha}=\widetilde{\alpha}\left(\overline{\mathscr{B}}_{n}\right)(12.10 .1)$ et l'on désigne par $\operatorname{Mod}\left(X_{\bar{\eta}}\right.$,fét, $\left.\mathfrak{o}_{n}^{\alpha}\right)$ la catégorie des $\mathfrak{o}_{n}^{\alpha}$-modules de $\alpha$ - $X_{\bar{\eta}, \text { fét }}$ et par $\operatorname{Mod}\left(\overline{\mathscr{B}}_{n}^{\alpha}\right)$ la catégorie des $\overline{\mathscr{B}}_{n}^{\alpha}$-modules de $\alpha-\widetilde{E}_{s}(12.11)$.

Comme $\overline{\mathscr{B}}_{n}$ est plat sur $\mathfrak{o}_{n}\left[4\right.$, III.9.2], le foncteur $\beta_{n}^{*}: \operatorname{Mod}\left(X_{\bar{\eta}, \text { fét }}, \mathfrak{o}_{n}\right) \rightarrow$ $\operatorname{Mod}\left(\overline{\mathscr{B}}_{n}\right)(7.13 .1)$ est exact et il induit un foncteur exact (12.26) que l'on note

$$
b_{n}: \operatorname{Mod}\left(X_{\bar{\eta}, \text { fét }}, \mathfrak{o}_{n}^{\alpha}\right) \rightarrow \operatorname{Mod}\left(\overline{\mathscr{B}}_{n}^{\alpha}\right) .
$$

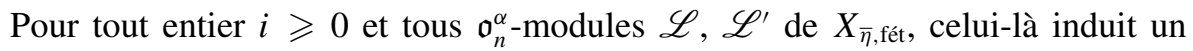
morphisme canonique (2.3.2)

$$
\operatorname{Ext}_{\mathfrak{o}_{n}^{\alpha}}^{i}\left(\mathscr{L}, \mathscr{L}^{\prime}\right) \rightarrow \operatorname{Ext}_{\overline{\mathscr{B}}_{n}^{\alpha}}^{i}\left(b_{n}(\mathscr{L}), b_{n}\left(\mathscr{L}^{\prime}\right)\right)
$$


Proposition 14.2. Soit $\mathbb{L}$ et $\mathbb{L}^{\prime}$ deux $\mathfrak{o}_{n}$-modules localement libres de type fini

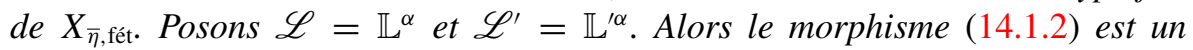
isomorphisme si $i=0,1$ et un monomorphisme si $i=2$.

Preuve. Le foncteur exact $\beta_{n}^{*}$ induit, pour tout entier $i \geqslant 0$, un morphisme canonique (2.3.2)

$$
\operatorname{Ext}_{\mathfrak{o}_{n}}^{i}\left(\mathbb{L}, \mathbb{L}^{\prime}\right) \rightarrow \operatorname{Ext}_{\mathscr{B}_{n}}^{i}\left(\beta_{n}^{*}(\mathbb{L}), \beta_{n}^{*}\left(\mathbb{L}^{\prime}\right)\right) .
$$

Comme $\mathbb{L}$ est un $\mathfrak{o}_{n}$-module localement libre de type fini de $X_{\text {fét, }}$, on a un isomorphisme canonique

$$
\beta_{n}^{*}\left(\mathscr{H}_{o m_{\mathfrak{o}_{n}}}\left(\mathbb{L}, \mathbb{L}^{\prime}\right)\right) \stackrel{\sim}{\rightarrow} \mathscr{H}_{o m_{\mathscr{B}_{n}}}\left(\beta_{n}^{*}(\mathbb{L}), \beta_{n}^{*}\left(\mathbb{L}^{\prime}\right)\right) .
$$

D'après (2.9.1) et (14.2.2), le morphisme (14.2.1) s'identifie au morphisme canonique induit $\operatorname{par} \beta_{n}^{*}$

$$
\mathrm{H}^{i}\left(X_{\bar{\eta}, \text { fét }}, \mathscr{H} o m_{\mathfrak{o}_{n}}\left(\mathbb{L}, \mathbb{L}^{\prime}\right)\right) \rightarrow \mathrm{H}^{i}\left(\widetilde{E}_{s}, \beta_{n}^{*}\left(\mathscr{H} o m_{\mathfrak{o}_{n}}\left(\mathbb{L}, \mathbb{L}^{\prime}\right)\right)\right) .
$$

On notera que $\mathscr{H} o m_{\mathfrak{o}_{n}}\left(\mathbb{L}, \mathbb{L}^{\prime}\right)$ est un $\mathfrak{o}_{n}$-module localement libre de type fini de $X_{\bar{\eta}, \text { fét }}$. Le morphisme (14.2.3) est donc un $\alpha$-isomorphisme pour $i=0,1$ et un $\alpha$-monomorphisme pour $i=2$ en vertu de 7.20. Par suite, il en est de même de (14.2.1).

Comme $\mathbb{L}$ est localement libre de type fini, on a deux suites spectrales (cf. 12.27)

$$
\begin{aligned}
\mathrm{E}_{2}^{i, j}=\operatorname{Ext}_{\mathfrak{o}}^{i}\left(\mathfrak{m}, \operatorname{Ext}_{\mathfrak{o}_{n}}^{j}\left(\mathbb{L}, \mathbb{L}^{\prime}\right)\right) & \Rightarrow \mathrm{E}^{i+j}=\operatorname{Ext}_{\mathfrak{o}_{n}^{\alpha}}^{i+j}\left(\mathscr{L}, \mathscr{L}^{\prime}\right) \\
\mathrm{E}_{2}^{i, j}=\operatorname{Ext}_{\mathfrak{o}}^{i}\left(\mathfrak{m}, \operatorname{Ext}_{\mathscr{\mathscr { B }}_{n}}^{j}\left(\beta_{n}^{*}(\mathbb{L}), \beta_{n}^{*}\left(\mathbb{L}^{\prime}\right)\right)\right) & \Rightarrow \mathrm{E}^{i+j}=\operatorname{Ext}_{\mathscr{\mathscr { B }}_{n}^{\alpha}}^{i+j}\left(b_{n}(\mathscr{L}), b_{n}\left(\mathscr{L}^{\prime}\right)\right)
\end{aligned}
$$

et les foncteurs exacts $\beta_{n}^{*}$ et $b_{n}$ induisent un morphisme de suites spectrales

$$
u=\left(u_{r}^{i, j}, u^{m}\right): \mathrm{E} \rightarrow \mathrm{E}^{\prime} .
$$

D'après 3.2(iii)-(iv), le morphisme canonique induit par (14.2.1)

$$
u_{2}^{i, j}: \operatorname{Ext}_{\mathfrak{o}}^{i}\left(\mathfrak{m}, \operatorname{Ext}_{\mathfrak{o}_{n}}^{j}\left(\mathbb{L}, \mathbb{L}^{\prime}\right)\right) \rightarrow \operatorname{Ext}_{\mathfrak{o}^{i}}^{i}\left(\mathfrak{m}, \operatorname{Ext}_{\mathscr{B}_{n}}^{j}\left(\beta_{n}^{*}(\mathbb{L}), \beta_{n}^{*}\left(\mathbb{L}^{\prime}\right)\right)\right)
$$

est un isomorphisme si $j=0,1$ et un monomorphisme si $i=0$ et $j=2$. D'après 2.6, le morphisme canonique $u^{m}$ (14.1.2) est un isomorphisme si $m=0,1$ et un monomorphisme si $m=2$.

Proposition 14.3. Soit un entier $m \geqslant 1$ et $\mathscr{L}_{m}$ un objet de $\operatorname{Mod}^{\mathrm{pff}}\left(X_{\bar{\eta}, \text { fét }}, \mathfrak{o}_{m}^{\alpha}\right)$ (13.14) tels que le morphisme (14.1.2) induit par $b_{m}$

$$
\operatorname{Ext}_{\mathfrak{o}_{m}^{\alpha}}^{i}\left(\mathscr{L}_{m}, \mathscr{L}_{m}\right) \rightarrow \operatorname{Ext}_{\mathscr{B}_{m}^{\alpha}}^{i}\left(b_{m}\left(\mathscr{L}_{m}\right), b_{m}\left(\mathscr{L}_{m}\right)\right)
$$


soit un isomorphisme pour $i=0,1$ et un monomorphisme pour $i=2$. Supposons qu'il existe une déformation $M$ de $b_{m}\left(\mathscr{L}_{m}\right)$ sur $\overline{\mathscr{B}}_{2 m}^{\alpha}$ (12.31(ii), 13.9). Alors :

(i) il existe une déformation $\mathscr{L}_{2 m}$ de $\mathscr{L}_{m}$ sur $\mathfrak{o}_{2 m}^{\alpha}$ telle que $b_{2 m}\left(\mathscr{L}_{2 m}\right)$ soit isomorphe à $M$ en tant que déformation;

(ii) le morphisme (14.1.2) induit par $b_{2 m}$

$$
\operatorname{Ext}_{\mathfrak{o}_{2 m}^{\alpha}}^{i}\left(\mathscr{L}_{2 m}, \mathscr{L}_{2 m}\right) \rightarrow \operatorname{Ext}_{\mathscr{\mathscr { B }}_{2 m}^{\alpha}}^{i}\left(b_{2 m}\left(\mathscr{L}_{2 m}\right), b_{2 m}\left(\mathscr{L}_{2 m}\right)\right)
$$

est un isomorphisme pour $i=0,1$ et un monomorphisme pour $i=2$.

Preuve. (i) On pose $I=p^{m} \mathfrak{o} / p^{2 m} \mathfrak{o}$ et $I^{\prime}=p^{m} \overline{\mathscr{B}} / p^{2 m} \overline{\mathscr{B}}$. En vertu de 13.8 , le foncteur $b_{2 m}$ induit un diagramme commutatif

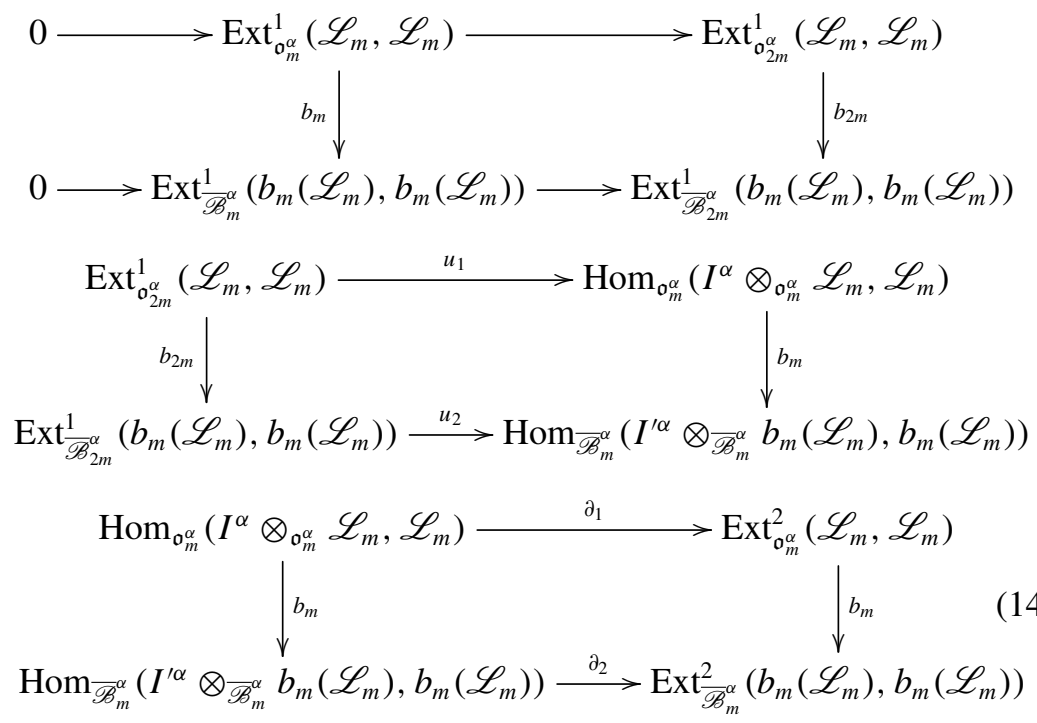

où la première (resp. seconde) ligne est la suite exacte (13.6.1) associée aux $\mathfrak{o}_{m}^{\alpha}$ modules $\mathscr{L}_{m}$ et $\mathscr{L}_{m}$ (resp. $\overline{\mathscr{B}}_{m}^{\alpha}$-modules $b_{m}\left(\mathscr{L}_{m}\right)$ et $b_{m}\left(\mathscr{L}_{m}\right)$ ). Notons $w_{1}$ (resp. $w_{2}$ ) l'isomorphisme (13.9.1) associé au $\mathfrak{o}_{m}^{\alpha}$-module plat $\mathscr{L}_{m}$ de $X_{\bar{\eta} \text {,fét }}$ (resp. $\overline{\mathscr{B}}_{m}^{\alpha}$ module plat $\left.b_{m}\left(\mathscr{L}_{m}\right)\right)$. On a alors $b_{m}\left(w_{1}\right)=w_{2}$.

Comme il existe une déformation $M$ de $b_{m}\left(\mathscr{L}_{m}\right)$ sur $\overline{\mathscr{B}}_{2 m}^{\alpha}, \partial_{2}\left(w_{2}\right)$ est nul d'après 13.7(i). La dernière flèche verticale de (14.3.3) est injective. Par suite, $\partial_{1}\left(w_{1}\right)=0$. Compte tenu de 13.7(i), il existe une déformation de $\mathscr{L}_{m}$ sur $\mathfrak{o}_{2 m}^{\alpha}$. En vertu de 13.7(ii), l'assertion résulte du fait que la première flèche verticale du diagramme (14.3.3) est un isomorphisme. 
(ii) Posons $\mathbb{L}_{m}=\tau_{!}\left(\mathscr{L}_{m}\right)$ et $\mathbb{L}_{2 m}=\tau_{!}\left(\mathscr{L}_{2 m}\right)$. D'après (12.25.4) et (12.26.1), l'hypothèse revient à dire que le morphisme induit par $\beta_{m}^{*}$

$$
\operatorname{Ext}_{\mathfrak{o}_{m}}^{i}\left(\mathbb{L}_{m}, \mathbb{L}_{m}\right) \rightarrow \operatorname{Ext}_{\mathscr{B}_{m}}^{i}\left(\beta_{m}^{*}\left(\mathbb{L}_{m}\right), \beta_{m}^{*}\left(\mathbb{L}_{m}\right)\right)
$$

est un isomorphisme pour $i=0,1$ et un monomorphisme pour $i=2$. De même, il suffit de démontrer la même propriété pour le morphisme induit par $\beta_{2 m}^{*}$

$$
\operatorname{Ext}_{\mathfrak{o}_{2 m}}^{i}\left(\mathbb{L}_{2 m}, \mathbb{L}_{2 m}\right) \rightarrow \operatorname{Ext}_{\mathscr{\mathscr { B }}_{2 m}}^{i}\left(\beta_{2 m}^{*}\left(\mathbb{L}_{2 m}\right), \beta_{2 m}^{*}\left(\mathbb{L}_{2 m}\right)\right) .
$$

Comme $\tau_{!}$est exact, $\mathbb{L}_{2 m}$ est une extension de $\mathbb{L}_{m}$ par $\mathbb{L}_{m}$. Par dévissage, il suffit de démontrer que le morphisme induit par $\beta_{2 m}^{*}$

$$
\operatorname{Ext}_{\mathfrak{o}_{2 m}}^{i}\left(\mathbb{L}_{m}, \mathbb{L}_{m}\right) \rightarrow \operatorname{Ext}_{\mathscr{B}_{2 m}}^{i}\left(\beta_{m}^{*}\left(\mathbb{L}_{m}\right), \beta_{m}^{*}\left(\mathbb{L}_{m}\right)\right)
$$

est un isomorphisme pour $i=0,1$ et un monomorphisme pour $i=2$. Rappelons que l'on a deux suites spectrales (13.5)

$$
\begin{aligned}
\mathrm{E}_{2}^{i, j} & =\operatorname{Ext}_{\mathfrak{o}_{m}}^{i}\left(\mathscr{T o r}_{j}^{\mathfrak{o}_{2 m}}\left(\mathbb{L}_{m}, \mathfrak{o}_{m}\right), \mathbb{L}_{m}\right) \Rightarrow \\
\mathrm{E}^{i+j} & =\operatorname{Ext}_{\mathfrak{o}_{2 m}}^{i+j}\left(\mathbb{L}_{m}, \mathbb{L}_{m}\right), \\
\mathrm{E}_{2}^{i, j} & =\operatorname{Ext}_{\mathscr{B}_{m}}^{i}\left(\mathscr{T}_{o r}^{\overline{\mathscr{B}}_{2 m}}\left(\beta_{m}^{*}\left(\mathbb{L}_{m}\right), \overline{\mathscr{B}}_{m}\right), \beta_{m}^{*}\left(\mathbb{L}_{m}\right)\right) \Rightarrow \\
\mathrm{E}^{i+j} & =\operatorname{Ext}_{\overline{\mathscr{B}}_{2 m}}^{i+j}\left(\beta_{m}^{*}\left(\mathbb{L}_{m}\right), \beta_{m}^{*}\left(\mathbb{L}_{m}\right)\right)
\end{aligned}
$$

et que le foncteur $\beta_{2 m}^{*}$ induit un morphisme de suites spectrales

$$
u=\left(u_{r}^{i, j}, u^{n}\right): \mathrm{E} \rightarrow \mathrm{E}^{\prime} .
$$

Comme $I \simeq \mathfrak{o}_{m}, I^{\prime} \simeq \overline{\mathscr{B}}_{m}$, on en déduit, pour tout entier $j \geqslant 2$, des isomorphismes

$$
\begin{aligned}
\mathscr{T}_{j}^{\mathfrak{o}_{2 m}}\left(\mathbb{L}_{m}, \mathfrak{o}_{m}\right) & \simeq \mathscr{T}^{\mathfrak{o}_{j-1} \boldsymbol{o}_{2 m}}\left(\mathbb{L}_{m}, \mathfrak{o}_{m}\right), \\
\mathscr{T}_{j}^{\overline{\mathscr{B}}_{2 m}}\left(\beta_{m}^{*}\left(\mathbb{L}_{m}\right), \overline{\mathscr{B}}_{m}\right) & \simeq \mathscr{T}_{\operatorname{or}_{j-1}}^{\overline{\mathscr{B}}_{2 m}}\left(\beta_{m}^{*}\left(\mathbb{L}_{m}\right), \overline{\mathscr{B}}_{m}\right) .
\end{aligned}
$$

Pour $j=1$, on a $\mathscr{T} r_{1}^{\mathfrak{o}_{2 m}}\left(\mathbb{L}_{m}, \mathfrak{o}_{m}\right) \simeq \mathbb{L}_{m}$ et $\mathscr{T}_{0}{\overline{\mathscr{B}}_{1}}_{2 m}\left(\beta_{m}^{*}\left(\mathbb{L}_{m}\right), \overline{\mathscr{B}}_{m}\right) \simeq \beta_{m}^{*}\left(\mathbb{L}_{m}\right)$. Le morphisme $u_{2}^{i, j}: \mathrm{E}_{2}^{i, j} \rightarrow \mathrm{E}_{2}^{i, j}$ s'identifie alors au morphisme (14.3.4). D'après 2.6 et l'hypothèse, le morphisme $u^{i}$ (14.3.6) est un isomorphisme si $i=0,1$ et un monomorphisme si $i=2$; d'où l'assertion.

THÉORÈmE 14.4. Soit $C$ une courbe propre et lisse sur $\bar{K}, \check{C}=C \otimes_{\bar{K}} \mathfrak{C}$ et $F$ un fibré vectoriel de Deninger-Werner sur $C$ (6.12(i)). Alors, il existe un trait $S^{\prime}$ fini sur $S$, un $S^{\prime}$-modèle semi-stable et régulier $X$ de $C(10.15)$ et un fibré vectoriel de Deninger-Werner et de Weil-Tate $\mathcal{F}$ sur $\check{\bar{X}}=X \times_{S^{\prime}} \check{\check{S}}_{\text {tel }}$ que $\mathcal{F}_{\bar{\eta}} \simeq F(6.3,10.4)$. 
Preuve. On fixe un entier $n \geqslant 1$. D'après 10.5 , quitte à remplacer $S$ par une extension finie, il existe un $S$-modèle semi-stable et régulier $X$ de $C$ et un fibré vectoriel de Deninger-Werner $\mathcal{F}$ sur $\check{\bar{X}}$ de fibre générique $F$ tel que l'on ait un isomorphisme

$$
\gamma_{n}: \sigma_{n}^{*}\left(\mathcal{F}_{n}\right) \stackrel{\sim}{\rightarrow} \beta_{n}^{*}\left(\mathbb{V}_{n}(\mathcal{F})\right)
$$

où $\mathbb{V}_{n}(\mathcal{F})$ est la $\mathfrak{o}_{n}$-représentation de $\pi_{1}(C, \bar{x})$ associée à $\mathcal{F}(6.7)$. On note $\mathbb{L}_{n}$ le $\mathfrak{o}_{n}$-module localement libre de type fini de $C_{\text {fét }}$ associé à $\mathbb{V}_{n}(\mathcal{F})$ (cf. (3.29.2)) et l'on pose $\mathscr{L}_{n}=\mathbb{L}_{n}^{\alpha}$.

On va démontrer par récurrence que pour tout entier $m \in\left\{2^{l} n \mid l \geqslant 0\right\}$, il existe un objet $\mathscr{L}_{m}$ de $\operatorname{Mod}^{\text {ptf }}\left(C_{\text {fét }}, \mathfrak{o}_{m}^{\alpha}\right)$ vérifiant l'hypothèse de 14.3 et un isomorphisme de $\overline{\mathscr{B}}_{m}^{\alpha}$-modules

$$
\gamma_{m}:\left(\sigma_{m}^{*}\left(\mathcal{F}_{m}\right)\right)^{\alpha} \stackrel{\sim}{\rightarrow} b_{m}\left(\mathscr{L}_{m}\right)
$$

tels que les isomorphismes $\left(\gamma_{m}\right)$ soient compatibles. L'assertion pour $m=n$ résulte de 14.2 et (14.4.1). Supposons que l'assertion soit vraie pour $m \geqslant n$ et démontrons-la pour $2 m$.

Le $\overline{\mathscr{B}}_{2 m}$-module $\sigma_{2 m}^{*}\left(\mathcal{F}_{2 m}\right)$ est une déformation de $\sigma_{m}^{*}\left(\mathcal{F}_{m}\right)$ sur $\overline{\mathscr{B}}_{2 m}$ (13.11(i)). On l'identifie à une déformation de $b_{m}\left(\mathscr{L}_{m}\right)$ sur $\overline{\mathscr{B}}_{2 m}^{\alpha}$ via le $\alpha$-isomorphisme $\gamma_{m}$ (14.4.2). D'après 14.3(i), il existe une déformation $\mathscr{L}_{2 m}$ de $\mathscr{L}_{m}$ sur $\mathfrak{o}_{2 m}^{\alpha}$ et un isomorphisme de $\overline{\mathscr{B}}_{2 m}^{\alpha}$-modules

$$
\gamma_{2 m}:\left(\sigma_{2 m}^{*}\left(\mathcal{F}_{2 m}\right)\right)^{\alpha} \stackrel{\sim}{\rightarrow} b_{2 m}\left(\mathscr{L}_{2 m}\right)
$$

compatible avec $\gamma_{m}$. En vertu de 13.15(ii) et 14.3(ii), $\mathscr{L}_{2 m}$ est un objet de $\operatorname{Mod}^{\text {ptf }}\left(C_{\text {fét }}, \mathfrak{o}_{2 m}^{\alpha}\right)$ et il satisfait l'hypothèse de 14.3.

Pour tout entier $m \geqslant 1$, choisissons un entier $l \geqslant 0$ tel que $2^{l-1} n<m \leqslant 2^{l} n$ et posons $\mathscr{L}_{m}=\mathscr{L}_{2^{l} n} \otimes_{\mathfrak{o}_{2 l}^{\alpha} n} \mathfrak{o}_{m}^{\alpha}$. On en déduit des isomorphismes compatibles

$$
\gamma_{m}:\left(\sigma_{m}^{*}\left(\mathcal{F}_{m}\right)\right)^{\alpha} \stackrel{\sim}{\rightarrow} b_{m}\left(\mathscr{L}_{m}\right) .
$$

On désigne par $\alpha-C_{\text {fét }}^{\mathbb{N}^{\circ}}\left(\right.$ resp. $\left.\alpha-\widetilde{E}_{s}^{\mathbb{N}^{\circ}}\right)$ la catégorie des $\alpha$-o-modules de $C_{\text {fét }}^{\mathbb{N}^{\circ}}$ (resp. $\left.\widetilde{E}_{s}^{\mathbb{N}^{\circ}}\right)$ (12.7). On pose $\breve{\mathfrak{o}}^{\alpha}=\widetilde{\alpha}(\breve{\mathfrak{o}})$ (resp. $\breve{\breve{\mathscr{B}}}^{\alpha}=\widetilde{\alpha}(\breve{\mathscr{B}})$ ) (12.10.1) et l'on désigne $\operatorname{par} \operatorname{Mod}\left(C_{\text {fét }}^{\mathbb{N}^{\circ}}, \breve{\mathfrak{o}}^{\alpha}\right)$ la catégorie des $\breve{\mathfrak{o}}^{\alpha}$-modules de $\alpha-C_{\text {fét }}^{\mathbb{N}^{\circ}}$ et par $\operatorname{Mod}\left(\breve{\mathscr{\mathscr { B }}}^{\alpha}\right)$ la catégorie des $\breve{\mathscr{B}}^{\alpha}$-modules de $\alpha-\widetilde{E}_{s}^{\mathbb{N}^{\circ}}$ (12.11). Posons $\breve{\mathcal{F}}=\left(\mathcal{F}_{m}\right)_{m \geqslant 1}$ et $\mathscr{L}=$ $\left(\mathscr{L}_{m}\right)_{m \geqslant 1} \in \mathbf{P}\left(\mathbf{M o d}^{\text {ptf }}\left(C_{\text {fét }}, \mathfrak{o}_{\bullet}^{\alpha}\right)\right)$ (13.16) que l'on considère aussi comme un objet $\operatorname{de} \operatorname{Mod}\left(C_{\text {fét }}^{\mathbb{N}^{\circ}}, \breve{\mathfrak{o}}^{\alpha}\right)$ (cf. 12.34). Le foncteur $\breve{\beta}^{*}$ induit un foncteur que l'on note (12.25)

$$
b: \operatorname{Mod}\left(C_{\text {fét }}^{\mathbb{N}^{\circ}}, \breve{\mathfrak{o}}^{\alpha}\right) \rightarrow \operatorname{Mod}\left(\breve{\mathscr{\mathscr { B }}}^{\alpha}\right) \text {. }
$$


Par l'équivalence de catégories $\operatorname{Mod}\left(\stackrel{\breve{\mathscr{B}}}{\alpha}^{\alpha}\right) \stackrel{\sim}{\rightarrow} \operatorname{P}\left(\operatorname{Mod}\left(\overline{\mathscr{B}}_{.}^{\alpha}\right)\right)$ (12.34.2), le $\stackrel{\breve{\mathscr{B}}}{\alpha}^{\alpha}$ module $b(\mathscr{L})$ correspond au système projectif $\left(b_{m}\left(\mathscr{L}_{m}\right)\right)_{m \geqslant 1}$. En vertu de 12.34, les isomorphismes compatibles (14.4.3) induisent un isomorphisme de $\breve{\mathscr{B}}^{\alpha}$ modules

$$
\gamma:\left(\breve{\sigma}^{*}(\breve{\mathcal{F}})\right)^{\alpha} \stackrel{\sim}{\rightarrow} b(\mathscr{L}),
$$

où $\breve{\sigma}$ est le morphisme de topos annelés (7.12.1). En vertu de 13.18, il existe un $\breve{\mathfrak{o}}$ module localement libre de type fini $\mathbb{L}$ de $C_{\text {fét }}^{\mathbb{N}^{\circ}}$ tel que $\mathbb{L}_{\mathbb{Q}} \simeq \mathscr{L}_{\mathbb{Q}}$ dans la catégorie $\operatorname{Mod}_{\mathbb{Q}}\left(C_{\text {fét }}^{\mathbb{N}^{\circ}}, \breve{o}\right)$. En vertu de 12.22, on en déduit par (14.4.4) un isomorphisme de $\breve{\mathscr{B}}_{\mathbb{Q}}$-modules

$$
\left(\breve{\sigma}^{*}(\breve{\mathcal{F}})\right)_{\mathbb{Q}} \stackrel{\sim}{\rightarrow}\left(\breve{\beta}^{*}(\mathbb{L})\right)_{\mathbb{Q}}
$$

d'où le théorème.

Corollaire 14.5. Tout fibré vectoriel de Deninger-Werner sur $\check{C}$ est de WeilTate (10.16).

PROPOSITION 14.6. La restriction du foncteur (10.18.1)

$$
\mathscr{V}_{\check{C}}: \mathfrak{V}_{\check{C}}^{\mathrm{WT}} \rightarrow \operatorname{Rep}_{\mathfrak{C}}^{\mathrm{cont}}\left(\pi_{1}(C, \bar{x})\right)
$$

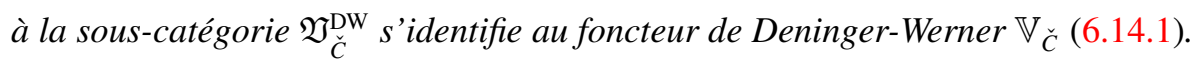

Preuve. Soit $F$ un fibré vectoriel de Deninger-Werner sur $\check{C}$. D'après 14.4 , quitte à remplacer $S$ par une extension finie, il existe un $S$-modèle semi-stable et régulier $X$ de $C$, et un fibré vectoriel de Deninger-Werner et de Weil-Tate $\mathcal{F}$ sur $\check{\bar{X}}$ de fibre générique $F$. En vertu de 8.21 , on en déduit un isomorphisme de $\mathfrak{C}$ représentations continues de $\pi_{1}(C, \bar{x})$ :

$$
\mathbb{V}_{\check{C}}(F) \stackrel{\sim}{\rightarrow} \mathscr{V}_{\check{C}}(F)
$$

Il reste à démontrer que celui-ci est fonctoriel en $F$. Soit $f: F \rightarrow F^{\prime}$ un morphisme $\mathfrak{V}_{\check{C}}^{\mathrm{DW}}$. En vertu de 5.8 et 14.4 , quitte à remplacer $S$ par une extension finie, il existe un $S$-modèle semi-stable et régulier $X$ de $C$ et deux fibrés vectoriels de Deninger-Werner et de Weil-Tate $\mathcal{F}$ et $\mathcal{F}^{\prime}$ sur $\check{\bar{X}}$ tels que $\mathcal{F}_{\overline{\bar{\eta}}} \simeq F$ et $\mathcal{F}_{\overline{\bar{\eta}}}^{\prime} \simeq$ $F^{\prime}$. D'après (5.13.1), $f$ s'étend en un morphisme de $\mathcal{F}$ à $\mathcal{F}^{\prime}$. La fonctorialité de (14.6.1) résulte alors de 8.21. 


\section{Appendice}

15.1. On se donne une catégorie abélienne $\mathscr{A}$ ayant suffisamment d'injectifs, un complexe borné inférieurement $K^{\bullet}$ de $\mathscr{A}$ et un objet $X$ de $\mathscr{A}$. Choisissons $L^{\bullet \bullet \bullet}$ une résolution injective de Cartan-Eilenberg de $K^{\bullet}$. La suite spectrale d'hypercohomologie [24, (0.11.4.3.2)]

$$
\mathrm{E}_{2}^{i, j}=\operatorname{Ext}_{\mathscr{A}}^{i}\left(X, \mathrm{H}^{j}\left(K^{\bullet}\right)\right) \Rightarrow \mathrm{E}^{i+j}=\mathrm{H}^{i+j}\left(\operatorname{RHom}_{\mathbf{D}(\mathscr{A})}\left(X, K^{\bullet}\right)\right)
$$

est la suite spectrale du bicomplexe $\operatorname{Hom}_{\mathscr{A}}\left(X, L^{\bullet \bullet \bullet}\right)$ associée à la filtration canonique $F_{\mathrm{II}}^{l}=\left(\sum_{i+j=n, l \geqslant i} \operatorname{Hom}_{\mathscr{A}}\left(X, L^{\bullet \bullet \bullet}\right)\right)_{n \in \mathbb{Z}}[24,0.11 .3 .2]$. Rappelons la construction de cette dernière. Pour la page 0 , on a $[47,5.6 .2]$

$$
\mathrm{E}_{0}^{i, j}=\operatorname{Hom}_{\mathscr{A}}\left(X, L^{j, i}\right)
$$

et le morphisme $d_{0}^{i, j}: \mathrm{E}_{0}^{i, j} \rightarrow \mathrm{E}_{0}^{i, j+1}$ est induit par la différentielle $L^{j, i} \rightarrow L^{j+1, i}$.

Notons, pour tous entiers $i$ et $l, \mathrm{Z}_{\mathrm{I}}^{l}\left(L^{\bullet, i}\right)\left(\operatorname{resp} . \mathrm{B}_{\mathrm{I}}^{l}\left(L^{\bullet, i}\right)\right.$, resp. $\left.\mathrm{H}_{\mathrm{I}}^{l}\left(L^{\bullet, i}\right)\right)$ le $l$ ième sous-groupe des cocycles (resp. des cobords, resp. groupe de cohomologie) du complexe $\left(L^{j, i}\right)_{j \in \mathbb{Z}}$. Le complexe $\left(Z_{\mathrm{I}}^{l}\left(L^{\bullet, i}\right)\right)_{i \geqslant 0}\left(\operatorname{resp} . \quad\left(\mathrm{B}_{\mathrm{I}}^{l}\left(L^{\bullet, i}\right)\right)_{i \geqslant 0}\right.$, resp. $\left.\left(\mathrm{H}_{\mathrm{I}}^{l}\left(L^{\bullet, i}\right)\right)_{i \geqslant 0}\right)$ est une résolution injective de $\mathrm{Z}^{l}\left(K^{\bullet}\right)\left(\right.$ resp. $\mathrm{B}^{l}\left(K^{\bullet}\right)$, resp. $\left.\mathrm{H}^{l}\left(K^{\bullet}\right)\right)$ (cf. [24, 0.11.4.2]). On a alors [47, 5.6.2]

$$
\mathrm{E}_{1}^{i, j}=\operatorname{Hom}_{\mathscr{A}}\left(X, \mathrm{H}_{\mathrm{I}}^{j}\left(L^{\bullet, i}\right)\right) .
$$

Le morphisme $d_{1}^{i, j}: \mathrm{E}_{1}^{i, j} \rightarrow \mathrm{E}_{1}^{i+1, j}$ est induit par la différentielle $\mathrm{H}^{j}\left(L^{\bullet, i}\right) \rightarrow$ $\mathrm{H}^{j}\left(L^{\bullet, i+1}\right)$. On en déduit

$$
\mathrm{E}_{2}^{i, j}=\operatorname{Ext}_{\mathscr{A}}^{i}\left(X, \mathrm{H}^{j}\left(K^{\bullet}\right)\right)
$$

15.2. On se donne de plus une catégorie abélienne $\mathscr{A}^{\prime}$ ayant suffisamment d'injectifs et un foncteur exact $F: \mathscr{A} \rightarrow \mathscr{A}^{\prime}$. Soit $K^{\prime \bullet}$ un complexe borné inférieurement de $\mathscr{A}^{\prime}$ et $g: F\left(K^{\bullet}\right) \rightarrow K^{\prime \bullet}$ un morphisme de complexes. On désigne par $\mathrm{E}^{\prime}$ la suite spectrale d'hypercohomologie

$$
\mathrm{E}_{2}^{i, j}=\operatorname{Ext}_{\mathscr{A}^{\prime}}^{i}\left(F(X), \mathrm{H}^{j}\left(K^{\prime \bullet}\right)\right) \Rightarrow \mathrm{E}^{\prime i+j}=\mathrm{H}^{i+j}\left(\operatorname{RHom}_{\mathbf{D}\left(\mathscr{A}^{\prime}\right)}\left(F(X), K^{\prime \bullet}\right)\right) .
$$

Choisissons une résolution injective de Cartan-Eilenberg $L^{\iota^{\bullet \bullet} \bullet}$ de $K^{\prime \bullet}$. On notera que $F\left(L^{\bullet \bullet \bullet}\right)$ est encore une résolution de Cartan-Eilenberg de $F\left(K^{\bullet}\right)$ (mais pas nécessairement injective). Le morphisme $g$ s'étend en un morphisme de bicomplexes ([24, 0.11.4.2], cf. aussi [9, XVII 1.2])

$$
G: F\left(L^{\bullet \bullet \bullet}\right) \rightarrow L^{\iota \bullet \bullet}
$$


On en déduit par le foncteur $F$ un morphisme de bicomplexes

$$
\operatorname{Hom}_{\mathscr{A}}\left(X, L^{\bullet \bullet \bullet}\right) \rightarrow \operatorname{Hom}_{\mathscr{A}^{\prime}}\left(F(X), L^{\prime \bullet \bullet \bullet}\right)
$$

et par suite un morphisme de suites spectrales [24, 0.11.2.3]

$$
u=\left(u_{r}^{i, j}, u^{n}\right): \mathrm{E} \rightarrow \mathrm{E}^{\prime} .
$$

15.3. Le morphisme $G(15.2 .1)$ s'identifie au morphisme $\left(u_{0}^{i, j}: \mathrm{E}_{0}^{i, j} \rightarrow \mathrm{E}_{0}^{i, j}\right)_{i, j}$ de $u$ (15.1.2) et il induit, pour tout entier $j$, un morphisme de complexes

$$
\left(F\left(\mathrm{H}_{\mathrm{I}}^{j}\left(L^{\bullet, i}\right)\right)\right)_{i \geqslant 0} \rightarrow\left(\mathrm{H}_{\mathrm{I}}^{j}\left(L^{\prime \bullet, i}\right)\right)_{i \geqslant 0} .
$$

Le morphisme composé, induit par celui-ci et le foncteur exact $F$,

$$
\begin{aligned}
& \operatorname{Hom}_{\mathscr{A}}\left(X, \mathrm{H}_{\mathrm{I}}^{j}\left(L^{\bullet, i}\right)\right) \rightarrow \operatorname{Hom}_{\mathscr{A}^{\prime}}\left(F(X), F\left(\mathrm{H}_{\mathrm{I}}^{j}\left(L^{\bullet, i}\right)\right)\right) \\
& \quad \rightarrow \operatorname{Hom}_{\mathscr{A}^{\prime}}\left(F(X), \mathrm{H}_{\mathrm{I}}^{j}\left(L^{\prime \bullet, i}\right)\right)
\end{aligned}
$$

s'identifie au morphisme $u_{1}^{i, j}$ de $u$ (15.1.3). Les morphismes $\left(u_{1}^{i, j}\right)_{i, j}$ induisent le morphisme $u_{2}^{i, j}$ (15.1.4)

$$
\operatorname{Ext}_{\mathscr{A}}^{i}\left(X, \mathrm{H}^{j}\left(K^{\bullet}\right)\right) \rightarrow \operatorname{Ext}_{\mathscr{A}^{\prime}}^{i}\left(F(X), \mathrm{H}^{j}\left(K^{\prime \bullet}\right)\right) .
$$

En outre, le morphisme de complexes $g$ (15.2) induit, pour tout entier $j$, un morphisme de groupes de cohomologie

$$
F\left(\mathrm{H}^{j}\left(K^{\bullet}\right)\right) \simeq \mathrm{H}^{j}\left(F\left(K^{\bullet}\right)\right) \rightarrow \mathrm{H}^{j}\left(K^{\bullet}\right) .
$$

Rappelons que le complexe $\left(\mathrm{H}_{\mathrm{I}}^{j}\left(L^{\bullet, i}\right)\right)_{i \geqslant 0}\left(\operatorname{resp} .\left(\mathrm{H}_{\mathrm{I}}^{j}\left(L^{\bullet \bullet, i}\right)\right)_{i \geqslant 0}\right)$ est une résolution injective de $\mathrm{H}^{j}\left(K^{\bullet}\right)$ (resp. $\left.\mathrm{H}^{j}\left(K^{\bullet}\right)\right)$. Le morphisme de complexes (15.3.1) étend le morphisme (15.3.3). Le morphisme $u_{2}^{i, j}$ (15.3.2) s'identifie alors au morphisme composé

$$
\operatorname{Ext}_{\mathscr{A}}^{i}\left(X, \mathrm{H}^{j}\left(K^{\bullet}\right)\right) \rightarrow \operatorname{Ext}_{\mathscr{A}^{\prime}}^{i}\left(F(X), F\left(\mathrm{H}^{j}\left(K^{\bullet}\right)\right)\right) \rightarrow \operatorname{Ext}_{\mathscr{A}^{\prime}}^{i}\left(F(X), \mathrm{H}^{j}\left(K^{\prime \bullet}\right)\right),
$$

où la première flèche est le morphisme canonique (2.3.2) induit par le foncteur exact $F$ et la seconde flèche est induite par (15.3.3).

15.4. Le morphisme $g$ et le foncteur exact $F$ induisent, pour tout entier $n$, un morphisme (2.3.2)

$$
\mathrm{E}^{n}=\operatorname{Ext}_{\mathbf{D}(\mathscr{A})}^{n}\left(X, K^{\bullet}\right) \rightarrow \mathrm{E}^{\prime n}=\operatorname{Ext}_{\mathbf{D}\left(\mathscr{A}^{\prime}\right)}^{n}\left(F(X), K^{\prime \bullet}\right),
$$

qui n'est autre que le morphisme $u^{n}$ de $u$. 
15.5. Reprenons les notations de 12.27. On considère le cas où $\mathscr{A}=\mathscr{A}^{\prime}=$ $\operatorname{Mod}(\mathfrak{o})$ et on prend pour $F$ le foncteur identique et pour $X$ le o-module $\mathfrak{m}$. Choisissons $I^{\bullet}\left(\right.$ resp. $I^{\bullet}$ ) une résolution injective de $N$ (resp. $\varphi^{*}(N)$ ) et posons $K^{\bullet}=\operatorname{Hom}_{A}\left(M, I^{\bullet}\right)$ et $K^{\prime \bullet}=\operatorname{Hom}_{A^{\prime}}\left(\varphi^{*}(M), I^{\bullet \bullet}\right)$. On a alors un morphisme de complexes $\varphi^{*}\left(I^{\bullet}\right) \rightarrow I^{\bullet}$ et par suite un morphisme de complexes $g: K^{\bullet} \rightarrow K^{\bullet \bullet}$. On notera que, dans ce cas, la suite spectrale (12.24.2) s'identifie à la suite spectrale (15.1.1). Appliquant ce qui précède, on en déduit le morphisme de suites spectrales $u$ (12.27.1) et la description des morphismes $u_{2}^{i, j}$ et $u^{n}$.

15.6. On considère le cas dual de 15.2. On se donne deux catégories abéliennes $\mathscr{A}$ et $\mathscr{A}^{\prime}$ ayant suffisamment d'objets projectifs, un foncteur exact $F: \mathscr{A} \rightarrow$ $\mathscr{A}^{\prime}$ et $X$ un objet de $\mathscr{A}$. Soit $K^{\bullet}$ un complexe borné supérieurement de $\mathscr{A}, K^{\prime \bullet}$ un complexe borné supérieurement de $\mathscr{A}^{\prime}$ et $g: K^{\bullet} \rightarrow F\left(K^{\bullet}\right)$ un morphisme de complexes. En remplaçant les résolutions injectives par les résolutions projectives, on construit un morphisme $u: \mathrm{E} \rightarrow \mathrm{E}^{\prime}$ entre les deux suites spectrales d'hypercohomologie

$$
\begin{aligned}
\mathrm{E}_{2}^{i, j} & =\operatorname{Ext}_{\mathscr{A}}^{i}\left(\mathrm{H}^{j}\left(K^{\bullet}\right), X\right) \Rightarrow \mathrm{H}^{i+j}\left(\operatorname{RHom}_{\mathbf{D}(\mathscr{A})}\left(K^{\bullet}, X\right)\right), \\
\mathrm{E}_{2}^{i, j} & =\operatorname{Ext}_{\mathscr{A}^{\prime}}^{i}\left(\mathrm{H}^{j}\left(K^{\prime \bullet}\right), F(X)\right) \Rightarrow \mathrm{H}^{i+j}\left(\mathbf{R H o m}_{\mathbf{D}\left(\mathscr{A}^{\prime}\right)}\left(K^{\prime \bullet}, F(X)\right)\right) .
\end{aligned}
$$

De même, les morphismes $u_{2}^{i, j}$ et $u^{n}$ s'identifient aux morphismes canoniques induits par le morphisme $g$ et le foncteur exact $F$.

15.7. Reprenons les notations de 13.5. On considère le cas où $\mathscr{A}=\operatorname{Mod}(A)$ et $\mathscr{A}^{\prime}=\operatorname{Mod}\left(A^{\prime}\right)$ et on prend pour $F$ le foncteur exact $\varphi^{*}$ et pour $X$ le $A$-module $J$. Choisissons $P^{\bullet} \rightarrow A_{0}$ (resp. $P^{\bullet} \rightarrow A_{0}^{\prime}$ ) une résolution projective de $A$-modules (resp. $A^{\prime}$-modules) et posons $K^{\bullet}=M_{0} \otimes_{A} P^{\bullet}$ et $K^{\bullet \bullet}=\varphi^{*}\left(M_{0}\right) \otimes_{A^{\prime}} P^{\prime \bullet}$. Comme $A_{0}^{\prime}=\varphi^{*}\left(A_{0}\right)$, on a alors un morphisme de complexes $P^{\bullet \bullet} \rightarrow \varphi^{*}\left(P^{\bullet}\right)$. Il induit un morphisme de complexes $g: K^{\prime \bullet} \rightarrow \varphi^{*}\left(K^{\bullet}\right)$. On notera que, dans ce cas, la suite spectrale (13.4.2) s'identifie à la suite spectrale (15.6.1). Appliquant ce qui précède, on en déduit le morphisme de suites spectrales $u$ (13.5.1) et la description des morphismes $u_{2}^{i, j}$ et $u^{n}$.

\section{Remerciements}

Ce travail fait partie de ma thèse préparée à l'université Paris-Sud. Je remercie sincèrement mon directeur de thèse Ahmed Abbes pour les nombreuses discussions, ainsi que pour ses commentaires et suggestions sur des versions préliminaires de ce texte. Je remercie Bhargav Bhatt de m'avoir signalé une erreur dans une version préliminaire de cet article. Je remercie également Michel Gros, Takeshi Tsuji et un rapporteur anonyme pour leurs commentaires et suggestions. 
Ce travail a bénéficié du soutien du programme ANR Théorie de Hodge $p$-adique et développements (ThéHopadD) ANR-11-BS01-005.

\section{Bibliographie}

[1] A. Abbes, 'Réduction semi-stable des courbes d'après Artin, Deligne, Grothendieck, Mumford, Saito, Winters...', in Courbes semi-stables et groupe fondamental en géométrie algébrique, Progress in Mathematics, 187 (Birkhäuser, Boston, 2000), 59-110.

[2] A. Abbes, Éléments de géométrie rigide: Volume I. Construction et étude géométrique des espaces rigides, (Springer, Berlin, 2010).

[3] A. Abbes and M. Gros, 'La suite spectrale de Hodge-Tate', Preprint, 2015, arXiv: 1509.03617.

[4] A. Abbes, M. Gros and T. Tsuji, The p-adic Simpson Correspondence, Annals of Mathematics Studies, 193 (Princeton University Press, Princeton, 2016).

[5] P. Achinger, ' $K(\pi, 1)$-neighborhoods and comparison theorems', Compos. Math. 151(10) (2015), 1945-1964.

[6] M. Artin, A. Grothendieck and J. L. Verdier, Théorie des topos et cohomologie étale des schémas, SGA 4, Tome 1, LNM, 269 (Springer, Berlin, 1972), Tome 2, LNM, 270 (Springer, Berlin, 1972); Tome 3, LNM, 305 (Springer, Berlin, 1973).

[7] S. Bosch, W. Lütkebohmert and M. Raynaud, Néron Models, (Springer, Berlin, 1990).

[8] N. Bourbaki, Algèbre commutative, (Hermann, Paris, 1985), chapitres 1-9.

[9] H. Cartan and S. Eilenberg, Homological Algebra, (Princeton University Press, Princeton, 1956).

[10] P. Deligne and J. S. Milne, 'Tannakian categories', in Hodge Cycles, Motives, and Shimura Varieties, LNM, 900 (Springer, Berlin, 1981), 101-228.

[11] M. Demazure and A. Grothendieck, Schémas en groupes, SGA3, LNM, 151-153 (Springer, Berlin, 1970).

[12] C. Deninger and A. Werner, 'Vector bundles and $p$-adic representations I', Preprint, 2003, arXiv:0309.273.

[13] C. Deninger and A. Werner, 'Vector bundles on $p$-adic curves and parallel transport', Ann. Sci. Éc. Norm. Supér. (4) 38(4) (2005), 553-597.

[14] H. P. Epp, 'Eliminating wild ramification', Invent. Math. 19(3) (1973), 235-249.

[15] G. Faltings, 'p-adic Hodge theory', J. Amer. Math. Soc. 1 (1988), 255-299.

[16] G. Faltings, 'Almost étale extensions', in Cohomologies p-adiques et applications arithmétiques. II, Astérisque, 279 (Société mathématique de France, 2002), 185-270.

[17] G. Faltings, 'A p-adic Simpson correspondence', Adv. Math. 198 (2005), 847-862.

[18] O. Gabber and L. Ramero, Almost Ring Theory, LNM, 1800 (Springer, Berlin, 2003).

[19] O. Gabber and L. Ramero, 'Foundations for Almost Ring Theory', Release 6.8, Preprint, 2014, arXiv:math/0409584.

[20] P. Gabriel, 'Des catégories abéliennes', Bull. de la S. M. F. 90 (1962), 323-448.

[21] D. Gieseker, 'Stable vector bundles and the frobenius morphism', Ann. Sci. Éc. Norm. Supér. (4) 6(1) (1973), 95-101.

[22] J. Giraud, Cohomologie non abélienne, (Springer, Berlin, 1971).

[23] A. Grothendieck, Revêtements étales et groupe fondamental, SGA 1, LNM, 24 (Springer, Berlin, 1971).

[24] A. Grothendieck and J. Dieudonné, 'Éléments de géométrie algébrique, III Étude cohomologique des faisceaux cohérents', Publ. Math. Inst. Hautes Études Sci. 11 (1961), 17 (1963). 
[25] A. Grothendieck and J. Dieudonné, 'Éléments de géométrie algébrique, IV Étude locale des schémas et des morphismes de schémas', Publ. Math. Inst. Hautes Études Sci. 20 (1964), 24 (1965); 28 (1966); 32 (1967).

[26] A. Grothendieck and J. A. Dieudonné, Éléments de géométrie algébrique I, Seconde édition, (Springer, Berlin, 1971).

[27] L. Illusie, Complexe cotangente et déformations I, LNM, 239 (Springer, Berlin, 1971).

[28] L. Illusie, 'Produits orientés', in Travaux de Gabber sur l'uniformisation locale et la cohomologie étale des schémas quasi-excellents, Astérisque, 363-364 (Société mathématique de France, 2014).

[29] K. Kato, 'Logarithmic structures of Fontaine-Illusie', in Algebraic Analysis, Geometry, and Number Theory (Baltimore, MD, 1988), 191-224.

[30] S. Lichtenbaum, 'Curves over discrete valuation rings', Am. J. Math. 90 (1968), 380-405.

[31] Q. Liu, Algebraic Geometry and Arithmetic Curves, (Oxford University Press, Oxford, 2002).

[32] Q. Liu, 'Stable reduction of finite covers of curves', Compos. Math. 142(01) (2006), 101-118.

[33] J. S. Milne, Etale Cohomology, Princeton Mathematical Series, Vol. 33 (Princeton University Press, Princeton, 1980).

[34] L. Moret-Bailly, 'Métriques permises', in Séminaire sur les pinceaux arithmétiques: la conjecture de Mordell, Astérisque, 129 (Société mathématique de France, 1985).

[35] D. Mumford, Abelian Variety, (Oxford University Press, Oxford, 1970).

[36] M. S. Narasimhan and C. S. Seshadri, 'Stable and unitary vector bundles on a compact Riemann surface', Ann. of Math. (2) 82 (1965), 540-567.

[37] M. Raynaud, 'Spécialisation du foncteur de Picard', Publ. Math. Inst. Hautes Études Sci. 38 (1970), 27-76.

[38] M. Raynaud, 'p-groupes et réduction semi-stable des courbes', in The Grothendieck Festschrift, vol. III, Progress in Mathematics, 88 (Birkhäuser, Boston, 1990), 179-197.

[39] M. Raynaud and L. Gruson, 'Critères de platitude et de projectivité', Invent. Math. 13(1) (1971), 1-89.

[40] T. Saito, 'Log smooth extension of a family of curves and semi-stable reduction', J. Algebraic Geom. 13(2) (2004), 287-322.

[41] P. Scholze, ' $p$-adic Hodge theory for rigid-analytic varieties', Forum Math., Pi 1(e1) (2013).

[42] J.-P. Serre, Groupes algébriques et corps de classes, (Hermann, Paris, 1975).

[43] C. Simpson, 'Higgs bundles and local systems', Publ. Math. Inst. Hautes Études Sci. 75 (1992), 5-95.

[44] J. Tate, 'p-divisible groups', in Proc. Conf. Local Fields (Driebergen, 1966) (Springer, Berlin, 1967), 158-183.

[45] J. Tate, 'Relations between $K_{2}$ and Galois cohomology', Invent. Math. 36 (1976), 257-274.

[46] J. Tong, 'Application d'Albanese pour les courbes et contractions', Math. Ann. 338 (2007), 405-420.

[47] C. A. Weibel, An Introduction to Homological Algebra, (Cambridge University Press, Cambridge, 1995).

[48] The Stacks Project Authors, Stacks Project, 2017, http://stacks.math.columbia.edu. 